\title{
Control of the abyssal ocean overturning circulation by mixing-driven bottom boundary layers
}

\author{
by \\ Henri Francois Drake \\ B.S., Haverford College (2015)
}

Submitted to the Department of Earth, Atmospheric, and Planetary Sciences in partial fulfillment of the requirements for the degree of

Doctor of Philosophy

at the

MASSACHUSETTS INSTITUTE OF TECHNOLOGY

and the

WOODS HOLE OCEANOGRAPHIC INSTITUTION

September 2021

(C)2021 Henri F. Drake.

All rights reserved.

The author hereby grants to MIT and WHOI permission to reproduce and to distribute publicly paper and electronic copies of this thesis document in whole or in part in any medium now known or hereafter created.

Author

Department of Earth, Atmospheric, and Planetary Sciences Massachusetts Institute of Technology August 02, 2021

Certified by

Raffaele Ferrari Cecil and Ida Green Professor of Oceanography Massachusetts Institute of Technology Thesis Supervisor

Accepted by

Glenn Flierl

Chairman, Joint Committee for Physical Oceanography Massachusetts Institute of Technology

Woods Hole Oceanographic Institution 


\title{
Control of the abyssal ocean overturning circulation by mixing-driven bottom
}

\section{boundary layers}

\author{
by \\ Henri Francois Drake \\ Submitted to the Department of Earth, Atmospheric, and Planetary Sciences \\ Massachusetts Institute of Technology \\ on August 02, 2021, in partial fulfillment of the \\ requirements for the degree of \\ Doctor of Philosophy
}

\begin{abstract}
An emerging paradigm posits that the abyssal overturning circulation is driven by bottom-enhanced mixing, which results in vigorous upwelling in the bottom boundary layer (BBL) along the sloping seafloor and downwelling in the stratified mixing layer (SML) above; their residual is the overturning circulation. This boundary-controlled circulation fundamentally alters abyssal tracer distributions, with implications for global climate.

Chapter 1 describes how a basin-scale overturning circulation arises from the coupling between the ocean interior and mixing-driven boundary layers over rough topography, such as the sloping flanks of mid-ocean ridges. BBL upwelling is well predicted by boundary layer theory, whereas the compensation by SML downwelling is weakened by the upward increase of the basin-wide strati fication, which supports a finite net overturning. These simulated watermass transformations are comparable to best-estimate diagnostics but are sustained by a crude parameterization of boundary layer restratification processes. In Chapter 2, I run a realistic simulation of a fracture zone canyon in the Brazil Basin to decipher the non-linear dynamics of abyssal mixing layers and their inter actions with rough topography. Using a hierarchy of progressively idealized simulations, I identify three physical processes that set the stratification of abyssal mixing layers (in addition to the weak buoyancy-driven cross-slope circulation): submesoscale baroclinic eddies on the ridge flanks, en hanced up-canyon flow due to inhibition of the cross-canyon thermal wind, and homogenization of canyon troughs below the level of blocking sills. Combined, these processes maintain a sufficiently large near-boundary stratification for mixing to drive globally significant BBL upwelling. In Chap ter 3, simulated Tracer Release Experiments illustrate how passive tracers are mixed, stirred, and advected in abyssal mixing layers. Exact diagnostics reveal that while a tracer's diapycnal motion is directly proportional to the mean divergence of mixing rates, its diapycnal spreading depends on both the mean mixing rate and an additional non-linear stretching term. These simulations sug gest that the theorized boundary-layer control on the abyssal circulation is falsifiable: downwelling in the SML has already been confirmed by the Brazil Basin Tracer Release Experiment, while an upcoming experiment in the Rockall Trough will confirm or deny the existence of upwelling in the BBL.
\end{abstract}

Thesis Supervisor: Raffaele Ferrari

Title: Cecil and Ida Green Professor of Oceanography

Massachusetts Institute of Technology 


\section{Acknowledgments}

Let me affirm that the freedom to work in science on one's own, with congenial colleagues, unfettered by supervision, with a scientific problem in one's mind when he goes to bed and when he awakes next morning, to be able to give undivided attention to unraveling some puzzle of nature, is a privilege beyond compare.

Henry Stommel (1982) [see the fascinating history by Oreskes |2021

In elementary school, students are taught that the The Scientific Method is a well-defined and objective process that ensures the reproducibility, falsifiability, and- eventually- progression of scientific knowledge. In actuality, each scientist develops their own individual method and the science that emerges is some non-linear combination of them all. This great diversity of scientific methods is particularly useful for problems regarding complex natural systems such as Earth's climate, which do not necessarily admit well defined or unique solutions and instead require insight, creativity, and storytelling. Here, I would like to acknowledge those who have most shaped my own approach to science.

Raffaele Ferrari, my doctoral advisor and mentor, shared with me (some of) his incredible knack for identifying interesting scientific problems, cutting them down to their essential ingredients, and using simple theories to cook up a coherent scientific narrative. His remarkable passion for ocean turbulence is truly infectious and I will miss being a part of the vibrant research group he leads. Sonya Legg, my mentor and future postdoctoral host, sparked my interest in ocean mixing with her story of towering internal waves breaking in the South China Sea (circa Alford et al., 2015) and later inspired many of my numerical modelling ideas. Jorge Sarmiento, Stephen Griffies, and Adele Morrison, my predoctoral advisors, for providing me with a tractable yet interesting project that exposed me to physical oceanography. Glenn Flierl, my instructor and committee member, taught me the value of analytical solutions to geophysical problems, and of the mathematical tools used to unearth them. Larry Pratt, Andy Thompson, and Alistair Adcroft encouraged me to think about abyssal mixing layers from different perspectives, which revealed unexpected insights and useful connections to other geophysical problems. From Jim Ledwell, Kurt Polzin, and Andreas Thurnherr, I learned to doubt everything (especially theory and numerical models, but also observations). My collaborators John Deutch, Alan Edelman, and Ron Rivest, inspired me to leverage my scientific expertise to engage in climate change policy and politics.

While I have acquired many different perspectives, traits, and skills from this long list of men 
tors, I also recognize that I have sampled from a strongly biased distribution. Both at MIT/WHOI and more generally, Physical Oceanography and Climate Science remain dominated by people like me: white cis-gendered men trained at elite American institutions (Bernard and Cooperdock, 2018, Ranganathan et al., 2021). The institutionalized exclusion of racialized (and/or otherwise minori tized) scientists must end; doing so requires the majority group in power to redevelop educational pathways, build sustainable and equitable collaborations with historically black and minority-serving institutions, and exhibit fearless leadership (Morris, 2021). I am also thankful for groups (and many of the individuals therein), such as Towards Diversity and Inclusion in EAPS (TIDE), the Society for Women in Marine Science (SWMS), and Mentoring Physical Oceanography Women to Increase Retention (MPOWIR) for supporting minoritized scientists and driving institutional change (Mouw et al., 2018). Moving forward, I pledge to collaborate with a more diverse team of scientists and to build more inclusive and accessible communities in pursuit of both scientific knowledge and social, environmental, and climate justice.

This material is based upon work supported by the National Science Foundation Graduate Research Fellowship under Grant No. 174530. I also acknowledge funding support from National Science Foundation Awards OCE-1536515 and OCE-1736109. This work was partially supported by MIT's Rosenblith Presidential Fellowship.

I am thankful for my colleagues in the MIT EAPS and MIT/WHOI programs, many of whom have become close friends and more than compensated for the inevitable frustrations of graduate student life with their care and laughter. Most of all, I am thankful for my collaborator, mentor, and partner Lyssa Freese, who teaches me to be more virtuous and human ${ }^{1}$.

\footnotetext{
${ }^{1}$...and about atmospheric chemistry, atmospheric radiation, air quality impacts, energy systems, environmental justice, economics, and environmental \& climate policy.
} 


\section{Contents}

\begin{tabular}{lll}
\hline & Introduction & 11
\end{tabular}

1.1 Background . . . . . . . . . . . . . . . . . . . . . . 11

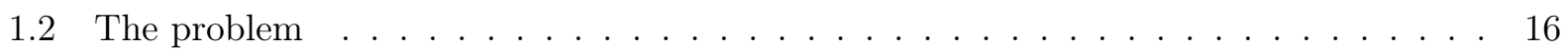

1.3 The thesis statement $\ldots \ldots \ldots \ldots \ldots$

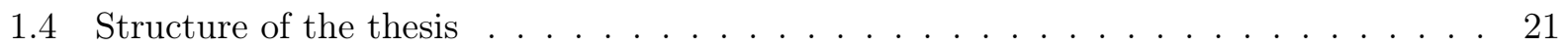

1.4 .1 Chapter $2 \mid$ basin-scale dynamics $\ldots \ldots \ldots \ldots \ldots \ldots$

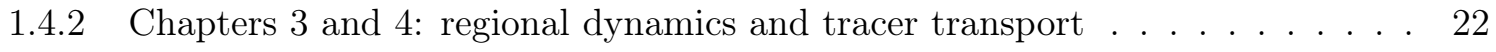

2 Abyssal circulation driven by near-boundary mixing: water mass transformations $\begin{array}{ll}\text { and interior stratification } & 25\end{array}$

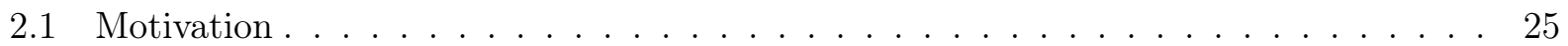

2.2 Theoretical Background $\ldots \ldots \ldots \ldots$

$2.2 .1 \quad$ Classical theories of abyssal stratification and circulation . . . . . . . . . . . 29

2.2 .2 Turning ocean mixing upside down $\ldots \ldots \ldots \ldots \ldots$

$2.2 .3 \quad$ A puzzling constraint from boundary layer theory . . . . . . . . . . . . . . . 30

$2.2 .4 \quad$ Boundary-interior exchange $\ldots \ldots \ldots \ldots \ldots \ldots \ldots \ldots$

2.2 .5 Dynamics controlling the interior abyssal stratification . . . . . . . . . . . . 32

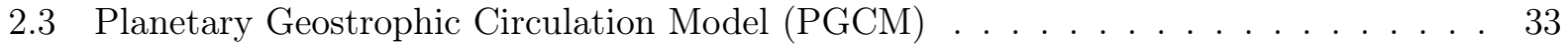

$2.3 .1 \quad$ Equations $\ldots \ldots \ldots \ldots \ldots$

$2.3 .2 \quad$ Geometry and boundary conditions . . . . . . . . . . . . . . . . . . 35

2.3 .3 Buoyancy forcing $\ldots \ldots \ldots \ldots$

2.3 .4 Dimensional parameters and scaling $\ldots \ldots \ldots$. . . . . . . . . . . . . . . 38

2.3 .5 Numerical implementation $\ldots \ldots \ldots \ldots$. . . . . . . . . . . . . . . 40

2.4 Abyssal Circulation Controlled By Mixing Layer Dynamics. . . . . . . . . . . . . . . 40

$2.4 .1 \quad$ Abyssal Mixing Layers and Deep Western Boundary Currents . . . . . . . . . 40 
$2.4 .2 \quad$ Depth-integrated and Overturning Circulations . . . . . . . . . . . . . . 41

$2.4 .3 \quad$ Partially-Compensating Watermass Transformations . . . . . . . . . . . . . . 43

2.5 Emulating the 3D PGCM with local 1D boundary layer models $\ldots . . . . .45$

2.5 .1 Boundary layer theory $\ldots \ldots \ldots \ldots \ldots \ldots$

2.5 .2 Emulator setup . . . . . . . . . . . . . . . . . . . . . 46

2.5 .3 Emulator evaluation . . . . . . . . . . . . . . . . . . . 46

2.6 The Effect of Variable Interior Stratification on the Abyssal Circulation $\ldots . . . .48$

2.6 .1 What sets the abyssal stratification? . . . . . . . . . . . . . . 48

2.6 .2 Effect of variable stratification on watermass transformations . . . . . . . . . 49

$2.6 .3 \quad$ Vertical extent of overturning set by ridge height $\ldots \ldots \ldots$. . . . . . . . 51

2.7 Comparison with realistic mid-ocean ridges $\ldots \ldots \ldots \ldots \ldots$

2.8 Classic recipes and new trends in abyssal cuisine $\ldots \ldots \ldots \ldots \ldots$

2.9 Discussion $\ldots \ldots \ldots \ldots \ldots \ldots \ldots$

3 Dynamics of eddying abyssal mixing layers over rough topography 63

3.1 Introduction . . . . . . . . . . . . . . . . . . . . . . . 63

3.2 Theory $\ldots \ldots \ldots \ldots \ldots \ldots$

$3.2 .1 \quad$ Slope-native equations $\ldots \ldots \ldots \ldots$

$3.2 .2 \quad$ Smooth planar slopes and steady 1D dynamics . . . . . . . . . . . . . . . . . 69

$3.2 .3 \quad$ Rough topography and eddy fluxes $\ldots \ldots \ldots$. . . . . . . . . . . . 72

3.3 Numerical model setup $\ldots \ldots \ldots \ldots$. . . . . . . . . . . . . . . . . 73

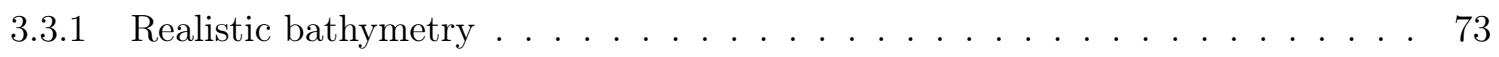

$3.3 .2 \quad$ Implementing the perturbation Boussinesq equations in the mean-slope coor dinate frame . . . . . . . . . . . . . . . . . . . . 74

$3.3 .3 \quad$ Forcing by observed bottom-enhanced turbulent mixing . . . . . . . . . . 75

3.3 .4 Parameter regime $\ldots \ldots \ldots \ldots \ldots \ldots$

3.3 .5 Numerics . . . . . . . . . . . . . . . . . . 76

$3.3 .6 \quad$ Hierarchy of progressively idealized simulations $\ldots \ldots \ldots$. . . . . . . . 77

3.4 Mixing-driven up-canyon flow, submesoscale turbulence, and stratification $\ldots . . .78$

3.5 Buoyancy budgets: mixing, mean flow, and eddies . . . . . . . . . . . . . . . . 81

3.6 Mixing-driven watermass transformations $\ldots \ldots \ldots \ldots \ldots$

3.7 Conclusions and Discussion $\ldots \ldots \ldots \ldots \ldots$ 
4 Diapycnal motion, diffusion, and stretching of tracers above rough topography 97

4.1 Introduction $\ldots \ldots \ldots \ldots \ldots \ldots \ldots$

4.2 Theory $\ldots \ldots \ldots \ldots$

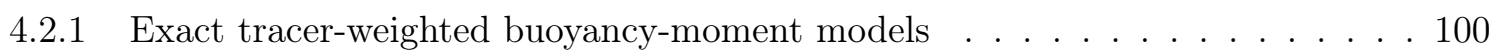

$4.2 .2 \quad$ Interpreting the tracer-weighted buoyancy moments $\ldots \ldots$. . . . . . . . . 102

4.3 Numerical methods overview: simulated TREs . . . . . . . . . . . . . . . . . . . 103

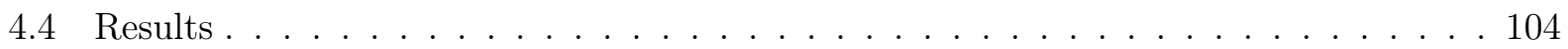

4.4 .1 Temporal evolution of the released tracer distributions . . . . . . . . . . 104

4.4.2 Diapycnal interior downwelling and near-boundary upwelling driven by bottomenhanced mixing . . . . . . . . . . . . . . . . . 106

4.4 .3 Bottom-enhanced diapycnal tracer spreading . . . . . . . . . . . . . . . 109

$4.4 .4 \quad$ Disentangling diapycnal stretching and contraction effects . . . . . . . . . . 109

4.5 Discussion and Conclusion $\ldots \ldots \ldots \ldots$

$\begin{array}{lll}5 & \text { Outlook } & 117\end{array}$

5.1 Observational evidence of mixing-driven bottom boundary layer upwelling . . . . . . 117

5.2 Tidal effects on abyssal mixing layers $\ldots \ldots \ldots$. . . . . . . . . . . . . . . . 119

5.3 Adjustment to non-local interior flows $\ldots \ldots \ldots$. . . . . . . . . . . 121

5.4 Towards predictive models of boundary-interior exchange . . . . . . . . . . . . . . 122

$5.5 \quad$ Sub-gridscale parameterization in general circulation models . . . . . . . . . . . . . . 124

5.6 The role of abyssal mixing layers in climate $\ldots \ldots$. . . . . . . . . . . . . 125

5.7 Towards an abyssal cookbook $\ldots \ldots \ldots \ldots \ldots \ldots \ldots \ldots$

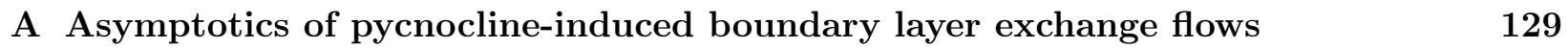

A.1 The planetary geostrophic equations in slope coordinates . . . . . . . . . . . . . . . 129

A.2 The creeping flow equations in slope coordinates _ . . . . . . . . . . . . 130

A.2.1 Boundary conditions . . . . . . . . . . . . . . . . . 133

A.2.2 Non-dimensional problem . . . . . . . . . . . . . . . . . 135

A.3 Regular perturbation expansion in aspect ratio $t \ldots \ldots \ldots$. . . . . . . . 135

A.3.1 $\mathcal{O}(1)$ solution $\ldots \ldots \ldots \ldots \ldots \ldots$

A.3.2 $\mathcal{O}(\epsilon)$ solution . . . . . . . . . . . . . . . . . . 136

\begin{tabular}{|cc}
\hline B Figures & 137
\end{tabular} 


\section{Chapter 1}

\section{Introduction}

The inclusion of an eddy-conductivity, $[\kappa]$, is a parametric way of including small scale vertical mixing processes into the theoretical model. Although this is a very common thing to encounter in the earlier oceanographical literature, it is a technique which present-day oceanographers are very reluctant to employ except as a last resort.

Robinson and Stommel (1959)

\subsection{Background}

The abyssal ocean, though far removed from Earth's atmosphere, plays a crucial role in modulating global climate. Its colossal mass and relatively high heat capacity allow it to store vast amounts of climatically-active tracers, such as heat, carbon, and nutrients (Sarmiento and Gruber, 2006). As forcing agents such as greenhouse gases and aerosols drive atmospheric changes, exchanges with this vast deep ocean reservoir tend to damp them ${ }^{1}$ (Schneider and Thompson 1981). For example, $90 \%$ of the heat trapped by anthropogenic forcing agents in the atmosphere has been stored in the ocean (Levitus et al. 2012). Including also carbon uptake, the uptake of atmospheric tracers by the deep ocean slows the transient rate of atmospheric global warming by up to $30 \%$, buying society more time for climate mitigation and adaptation (Figure 1-1; Drake et al. 2020b). In the absence of ocean mixing or circulation, these tracer exchanges would occur on an excessively long timescale set by the slow rate of molecular diffusion, e.g. $\tau=H^{2} / \kappa_{M}=\frac{(2000 \mathrm{~m})^{2}}{1.4 \times 10^{-7} \mathrm{~m}^{2} / \mathrm{s}} \approx 1 \mathrm{Myr}$ (for heat), much too slow to play an active role in modulating anthropogenic climate change.

\footnotetext{
${ }^{1}$ Although these exchanges tend to have a damping effect in isolation, interactions between several tracers, coupling with atmospheric dynamics, and other complexities can result in various system instabilities, some of which support multiple physical climate equilibria (see the original Stommel 1961, or a modern review by Weijer et al. 2019).
} 


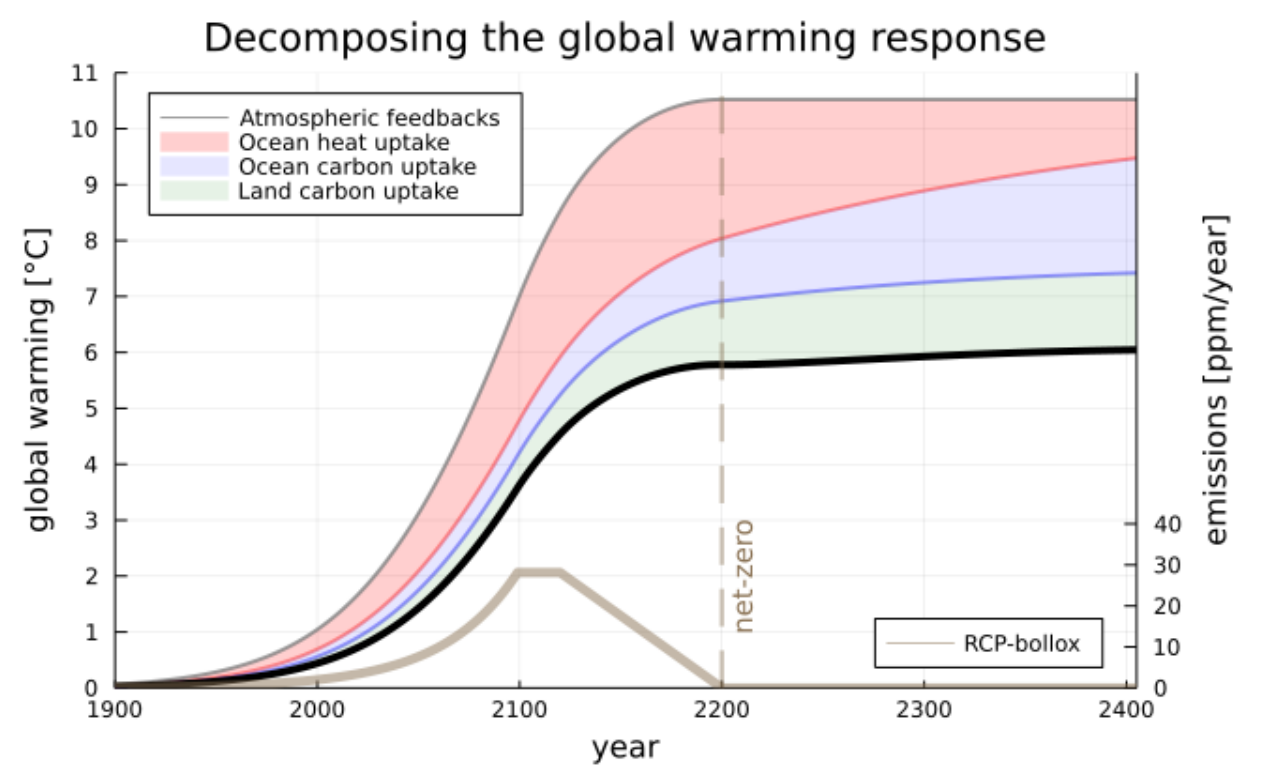

Figure 1-1: Decomposing the global warming response (solid black line) to a hypothetical highemissions scenario (RCP-bollox; thick brown line) in a simple climate model (Drake et al. 2020b, based on Joos et al. 2013 and Geoffroy et al. 2012). The thin grey line (left axis) shows the hypothetical equilibrium response to the instantaneous radiative forcing in a given year (i.e. the adjustment due to atmospheric feedbacks alone). Including heat uptake in the deep ocean (thin red line) delays $20-40 \%$ of the warming, but does not change the eventual equilibrium warming. By contrast, carbon uptake by the deep ocean progressively depletes the atmospheric stock, such that its cooling effect grows over time (thin blue line). Carbon uptake by the terrestrial biosphere removes a slightly smaller fraction of the atmospheric carbon stock, further reducing the greenhouse effect and bringing the total warming down to its actual level of about $6{ }^{\circ} \mathrm{C}$ (solid black line). Due to the compensating time-dependencies of ocean carbon and heat uptake, global warming stops if and only if carbon emissions reach net-zero (dashed line).

The observed rich spatial structure and temporal evolution of oceanic tracers, however, im plies the existence of strong mean flows and turbulence (Broecker and Peng, 1982). Meridional hydrographic sections across entire ocean basins, for example, reveal tongues of dense high oxygen (recently ventilated) waters that penetrate into the abyss (below a depth of $2000 \mathrm{~m}$ ) off the coast of Antarctica and lose oxygen due to respiration as they flow northwards (Talley, 2013a). Since these abyssal density classes outcrop to the surface only in the Southern Ocean regions where they are formed (i.e., diapycnal downwelling), their equal and opposite destruction (i.e., diapycnal up welling) must occur within the abyss itself. A small fraction of this upwelling can occur due to geothermal heating, and is further modulated by interactions between isopycnal stirring and seawa ter's non-linear equation of state (McDougall, 1989; Klocker and McDougall, 2010). The majority of abyssal waters, however, must be lightened by a mechanical process through which the fluid's kinetic energy is converted into raising its potential energy (Winters et al., 1995). The most likely 
energy source for mixing is turbulent breaking of internal waves, themselves ultimately sourced by some combination of astronomical tidal forcing, direct wind forcing, or topographic interactions with wind-forced geostrophic currents (Wunsch and Ferrari, 2004). High-mode internal waves break due to shear and gravitational instabilities; low-mode internal waves, on the other hand, can either break due to critical reflections at topography or can transfer their energy to unstable higher modes by topographic scattering and non-linear wave-wave interactions (see Whalen et al. 2020]s recent review of mixing by internal waves). While the detailed attribution of abyssal turbulence to the various energy sources remains an interesting unsolved problem, we are here most interested in the implications of the observed turbulence patterns for the large-scale overturning circulation; indeed, most of the theoretical arguments are agnostic as to the origin of abyssal mixing ${ }^{2}$

Turbulent flows, such as those caused by breaking internal waves, distort tracer distributions $\phi$ and thus modulate the tracer transport by larger-scale mean flows. In an integral sense, tur bulent advection (by $\mathbf{u}^{\prime}$ ) enhances tracer gradients $\nabla \phi$ by orders of magnitude locally (turbulent production) and dramatically accelerates the rate at which tracer variance is destroyed by molecular diffusion (turbulent dissipation) relative to the laminar case (mean dissipation),

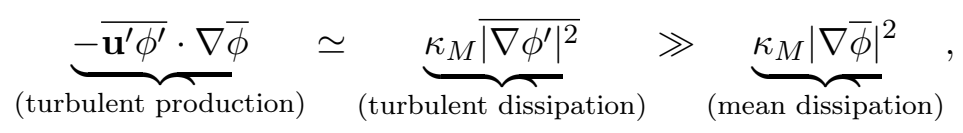

where the overline represents an average over many turbulent events (e.g. as a spatial average over the scale of a climate model's grid cell) and primes denote fluctuations away from that average. It is thus tempting to extend the molecular diffusion analogy to macroscopic scales and parameterize

\footnotetext{
${ }^{2}$ It is nonetheless worth mentioning two alternate abyssal recipes which depart from the mainstream internal wave paradigm. Following Munk's leading speculation of "boundary mixing", Armi (1978) posits that turbulent bottom boundary layer processes (e.g. shear instability induced by no-slip boundary condition)- produce the required mixing (e.g. Armi and D'Asaro, 1980, D'Asaro, 1982) and that their effect on scalars is then communicated to the interior by topographic separation or lateral eddy transport (see Armi, 1979a). Bryden and Nurser (2003) propose yet another version of the "boundary mixing" recipe, through which mixing associated with deep overflows over hydraulically critical sills (and associated hydraulic jumps) sequentially modify abyssal water properties and produce the observed abyssal stratification. While large mixing rates have indeed been observed surrounding the sills of several straits (e.g. Polzin et al., 1996, Alford et al. 2013), their total contribution to global watermass transformations have not yet been quantified. Neither of these processes explain the widespread in-situ observations (Waterhouse et al., 2014) of large dissipation rates that extend several hundreds of meters above rough topographic features (which could also be considered "boundary mixing"), whose dynamical characteristics are strongly suggestive of internal waves (Polzin, 2004, 2009, Whalen et al., 2020, Lavergne et al. 2020). Thus, however important these additional poorly-quantified processes may be, they operate only in addition to the internal wave mixing that drives a net upwelling of $10-35 \mathrm{~Sv}$ (de Lavergne et al., 2016c) comparable to the 21-27 Sv of Antarctic Bottom Water that fills the global abyss from the Southern Ocean (Talley et al. 2003).
} 
the mean turbulent tracer flux as an enhanced diffusion acting on mean tracer gradient $t^{3}$

$$
\overline{\mathbf{u}^{\prime} \phi^{\prime}} \approx-\kappa_{T} \nabla \bar{\phi}
$$

although the challenge is then to find the appropriate value of $\kappa_{T}$ for the problem at hand. Since the turbulent diffusivity is the result of macroscopic turbulent flow properties, the same value is generally used for all tracers. Mixing by large-scale baroclinic eddies (or "stirring") is orders of magnitude stronger than the turbulent mixing described above, but is at leading order confined by stratification and planetary rotation to act along buoyancy surfaces (i.e. quasi-adiabatic) (Montgomery, 1940, Redi, 1982, Gent and McWilliams, 1990; Young, 2011); hereafter, I refer to small-scale mixing across buoyancy surfaces as "mixing" $\left(\kappa_{T}\right.$, hereafter just $\left.\kappa\right)$ by "turbulence" and mixing along buoyancy surfaces as "stirring" by "eddies". Turbulent mixing is the star of this thesis, but we will find that "turbulence" and "eddies" are not independent of each other and must be considered together. The original theories of a mixing-driven abyssal circulation were developed by Stommel and his collaborators Arons and Faller (Stommel, 1957; Stommel et al., 1958; Stommel, 1958; Stommel and Arons, 1959a|b). Following the success of his upper-ocean theory for wind-driven gyres (Stommel 1948, still considered to be generally correct), he describes a horizontal abyssal circulation in which abyssal water columns are stretched from above by mixing-driven upwelling $\bar{w}$ at a depth $z$ (with $\bar{w}=0$ at a flat seafloor $z=0$ ) and thus the vertically-integrated meridional flow $\bar{V} \stackrel{\text { def }}{\equiv} \int_{0}^{z} v \mathrm{~d} z$ must be polewards $(\bar{V} f \geq 0)$ to conserve linear vorticity on a $\beta$-plane:

$$
\beta \bar{V}=f \bar{w} .
$$

He also applies his ideas of western-intensification to the abyss, predicting the existence of frictional Deep Western Boundary Currents that would close this abyssal gyre circulation by connecting the localized origins of bottom water to their broadly distributed fate via upwelling. One of the major caveats of Stommel's model is that the parameterized mixing rate is poorly justified, and is qualitatively tuned by fitting an early theory for the structure of the thermocline to observations (Robinson and Stommel, 1959).

In his ground-breaking paper, Munk (1966) proposes a simple recipe to explain the relationship

\footnotetext{
${ }^{3}$ Many have cautioned against relying on this poorly-justified parameterization, and have advocated for directly specifying the turbulent flux itself (e.g. Polzin, 2009); in practice, however, most existing parameterizing are only for thermodynamic tracer fluxes (e.g. buoyancy or temperature), such that parameterizing the flux of other tracers still makes use of the diffusive closure (e.g. Melet et al. 2013).
} 
between the inferred upwelling of abyssal waters (required by conservation of mass to balance observed downwelling at high latitudes) and the observed deep ocean stratification: upwelling of dense water across buoyancy surfaces is balanced by mixing of buoyancy downwards,

$$
\bar{w} \bar{b}_{z}=\partial_{z}\left(\kappa \bar{b}_{z}\right)
$$

Assuming a uniform upwelling $\bar{w}$ and mixing $\kappa$, this vertical advection-diffusion balance admits ex ponential solutions; given the exponential decay scale $\delta=\kappa / \bar{w}=1000 \mathrm{~m}$ fit to observations, closing the abyssal overturning circulation with a globally-averaged upwelling $\bar{w} \simeq 1 \mathrm{~cm} /$ day (inferred from the rate of dense water formation in the Southern Ocean), the recipe requires the now canonica 4 value of $\kappa \simeq 10^{-4} \mathrm{~m}^{2} / \mathrm{s}$ (or $1 \mathrm{Munk}$ ).

In the following decades, several methods for inferring in-situ oceanic $\kappa$ values are developed (see brief review in Chapter 4.1) and the race is on to confirm or deny Munk (1966)'s required abyssal mixing rate. The most common approaches are indirect variance budget methods that estimate turbulent buoyancy fluxes based on microstructure (velocity shear and temperature variances) or finestructure (internal wave strain/shear) observables. Combined with a down-gradient closure (1.2) These indirect observations reveal small mixing rates of $\kappa=\mathcal{O}(0.1$ Munks $)$ in the deep interior and much larger mixing rates of $\kappa=\mathcal{O}(100$ Munks $)$ above rough abyssal topography, such as the midocean ridges (Polzin et al. 1997). This general pattern is qualitatively (but not quantitatively) confirmed by more direct estimates of the mixing rates from purposeful tracer release experiments (Ledwell et al., 1993, 2000), in which a filtered tracer equation is inverted to estimate the effective $\kappa$. These observations of bottom-enhanced mixing over rough topography are also consistent with bottom-up theories of internal wave generation, propagation, and breaking (MacKinnon et al., 2017, Whalen et al., 2020), which are commonly used to extrapolate estimates of mixing rates globally (St. Laurent and Garrett, 2002, Polzin, 2009, Lavergne et al., 2020).

Combined with hydrographic measurements of the buoyancy field, three-dimensional maps of mixing rates provide estimates of the turbulent vertical buoyancy flux divergence, which is thought to dominate the total turbulent flux divergence because density surfaces are relatively flat outside of thin boundary layers. In steady state, these estimates of diabatic forcing by turbulent mixing are directly related to the velocity normal to a buoyancy surface, which can be integrated over buoyancy surfaces to estimate large-scale cross-buoyancy (or "diapycnal") transports (Marshall

${ }^{4}$ The mixing rate estimated by Robinson and Stommel| (1959)'s thermocline theory and used to force Stommel and Arons (1959b)'s abyssal circulation model was remarkably similar at 0.85 Munks. 
et al. 1999). While observed buoyancy flux profiles generally appear to increase towards to the bottom in the abyssal ocean (at least where they are large over rough topography St. Laurent et al., 2001; Waterhouse et al. 2014), the buoyancy flux must go to zero within a thin bottom boundary to satisfy the insulating bottom boundary condition (Ferrari et al., 2016); the resulting net diabatic abyssal overturning circulation is the small residual of vigorous upwelling in the Bottom Boundary Layer (BBL) and a partially-compensating downwelling in the Stratified Mixing Layer (SML) above. Like Munk's original balance, this watermass transformation framework is a useful diagnostic tool (Walin 1982; see also recent review by Groeskamp et al. 2017): given the mixing and stratification, one can diagnose the diapycnal transport (Ferrari et al., 2016, McDougall and Ferrari, 2017); given the stratification and diapycnal transport, one can invert for the mixing (Holmes et al., 2018).

\subsection{The problem}

The modern conceptual model of the abyssal circulation, described above, has emerged largely from diagnostic studie 5 5cDougall, 1989, Klocker and McDougall, 2010; Ferrari et al., 2016; de Lavergne et al., 2016b; McDougall and Ferrari, 2017; Holmes et al., 2018); reliable reconstruction of past cir culations or prediction of future circulations, however, requires prognostic dynamical models. Fol lowing Stommel (1958) and Munk (1966), most idealized dynamical models of an abyssal circulation driven by boundary mixing assume flat bottoms and vertical sidewalls (Marotzke, 1997; Scott and Marotzke, 2002; Samelson, 1998; Callies and Marotzke, 2012); whether these models parameterize boundary-enhanced mixing as a horizontal enhancement towards the sidewalls or vertical enhance ment towards the flat bottom, they result in unrealistic boundary flows and interior stratifications (Mashayek et al., 2015). Another approach has been to generalize Stommel (1958)-like models to allow both the direct effects of mid-ocean ridge topography on the flow and indirect effects such as enhanced mixing-driven upwelling; the resulting one-and-a-half or two-layer systems, however, do not resolve both the bottom-enhancement of the diffusivity (driving downwelling) and the buoyancy flux convergence in the BBL (driving upwelling) (Spall, 2001; Andersson and Veronis, 2004, Marchal and Nycander, 2004; Katsman, 2006).

Recognizing the importance of the sloping sidewalls, Ferrari et al. (2016) simulate the flow

\footnotetext{
${ }^{5}$ In my opinion, this kinematic approach has dramatically overemphasized the role of "hypsometric" effects in controlling the abyssal circulation. For example, de Lavergne et al. (2016c) and de Lavergne et al. (2017)'s claims that the "incrop area" drives the net diabatic overturning gives the impression that the overturning is set by topography (effectively fixed), while in reality incrop areas vary largely due to changes in the stratification (a prognostic variable, which itself varies in response to the buoyancy flux).
} 
in a bowl-shaped basin with a circumpolar channel in the South and force an abyssal circulation with a prescribed bottom-enhanced turbulent buoyancy flux profile (which goes to zero over the bottom few hundred meters to satisfy the no-flux bottom boundary condition). The simulated overturning features a realistic abyssal stratification and overturning circulation, which is the result of broad downwelling in the interior SML and upwelling along the sloping BBLs on the sidewalls of the basin. However, the imposed buoyancy flux (inspired by observations) is fixed, omitting the influence of stratification on the buoyancy flux and thus effectively prescribing the anticipated diabatic overturning. Neverthless, the qualitative consistency of the solution with observations demonstrates that sloping sidewalls are a key element of the abyssal circulation. Callies and Ferrari (2018b) run similar- but even more idealized- simulations where they instead prescribe the bottomenhanced diffusivity, and allow both the buoyancy field and turbulent buoyancy flux to co-evolve. The resulting circulation is similar to that described by Ferrari et al. (2016), although the emerging sloping boundary layer flows are now prognostic, well-resolved by their use of terrain-following coordinate, and can be qualitatively (but not quantitatively) understood using classical sloping boundary layer theory

Sloping boundary layer theory is an idealized framework for studying the dynamics of rotating and stratified flows along an infinitely sloping seafloor, which can be arise either through mixing ("spin-up") or in response to an imposed along-slope external flow ("spin-down" or "Ekman arrest") (see review by Garrett et al., 1993). In the classical "spin-up" problem, which I focus on in this thesi:7 turbulent mixing forces buoyancy surfaces to plunge downwards to meet the insulating ("no flux") boundary condition at the seafloor. The resulting buoyancy force drives a flow up the slope, which is known as the "secondary" circulation (where "primary" circulation refers to the along-slope flow). Callies and Ferrari (2018b) argue that the strength of the net overturning circulation in their three-dimensional general circulation model is well-predicted by one-dimensional sloping boundary layer dynamics. Extrapolated to the global ocean, these boundary layers produce a realistically large net overturning circulation (and BBL upwelling and SML downwelling consistent with diagnostic estimates of watermass transformations).

Mixing only drives efficient watermass transformations if it acts upon stratified water; paradox

\footnotetext{
${ }^{6}$ Huang and Jin (2002) describe similar simulations (restricted to just the Brazil Basin), although their realistic configuration obscures the fundamental aspects of the circulation and does not resolve the thin bottom boundary layers on the mid-ocean ridge.

${ }^{7}$ See a discussion of the role of "spin-down" and the competition between "spin-up" and "spin-down" in Outlook Section 5.3 For a quite different but complementary discussion of sloping boundary layers in the coastal ocean context, see (Trowbridge and Lentz, 2018).
} 
ically, however, these watermass transformations also tend to homogenize stratified water. What, then, maintains the strong near-bottom stratification that sustains large watermass transformations at equilibrium? The restratification dynamics of sloping bottom boundary layers is a particularly controversial topic (Armi, 1978, Garrett, 1979; Armi, 1979b, Garrett, 1990, Callies, 2018). A number of processes are known to affect the near-boundary stratification: the cross-slop 88 "secondary" cir culation (Garrett, 1990), restratification by bottom-intensified submesoscale baroclinic instabilities (Wenegrat et al., 2018; Callies, 2018), and the inhibition of along-slope momentum by narrowlyseparated canyon walls (Dell, 2013, Ruan and Callies, 2020). However, the detailed dynamics and relative importance (both regionally and globally) of these processes are poorly understood. Prob lematically, all of these processes also occur well below the grid scale of global circulation models, so their effects are thus either omitted or must be parameterized.

Callies and Ferrari (2018b)'s conceptual and numerical model of the abyssal overturning circu lation, and their interpretation of it in terms of sloping boundary layer theory, though enticing, is vulnerable to four major criticisms:

1. One-dimensional boundary layer theory predicts a vanishingly small net up-slope transport of $\kappa_{\mathrm{BG}} \cot \theta L \approx 0.5 \mathrm{~Sv}$ (for weak background mixing rates of $\kappa_{\mathrm{BG}}=10^{-5} \mathrm{~m}^{2} / \mathrm{s}$ and typical midocean ridge slopes of $\tan (\theta)=2 \times 10^{-3}$ ), unrealistically confining finite transformations to the base of the mid-ocean ridge where the compensating SML downwelling vanishes.

2. Their predictive model relies upon a poorly-justified parameterization of friction, a placeholder for "the more complicated and incompletely understood turbulent dynamics of real boundary regions" that act to maintain a realistic near-bottom stratification.

3. While they parameterize the indirect effects of rough topography on mixing by breaking internal waves, they do not account for direct effects of multi-scale topographic roughnesssuch as steering and blocking- on the boundary layer flows themselves, which are evident from both observations (Armi, 1978; Ledwell et al., 2000; Thurnherr and Speer, 2003) and previous modelling studies (Ruan and Callies, 2020; Dell and Pratt, 2015).

\footnotetext{
${ }^{8}$ Terminology: Throughout the thesis I follow the convention of sloping boundary layer theory Garrett et al. 1993) in referring to the direction parallel to the large-scale topographic slope as "cross-slope", the direction normal to the local topographic tangent-plane as the "slope-normal", and the third orthogonal direction is the "along-slope" direction. In general, the large-scale slope refers to the slope of mid-ocean ridges and I thus occasionally use the terms "cross-ridge" or "along-ridge". In Chapter 3 , I re-introduce smaller-scale topographic features such as fracture zone canyons; it is important to note that the "cross-slope" direction refers to the large-scale slope of the mid-ocean ridge, and not necessarily the local "cross-isobath" direction, which is scattered by the rough topography. Since fracture zone canyons are typically oriented perpendicular to mid-ocean ridges, I instead refer to the "along-canyon" (三cross-ridge) and "cross-canyon" (三 along-ridge) directions to avoid confusion (Thurnherr et al., 2005).
} 
4. The main prediction of the theory- vigorous mixing-driven diapycnal upwelling in the BBLis not yet falsifiable with available observations.

These criticisms of the emerging theory of boundary layer control on the abyssal circulation present major open questions; they also provide a blueprint for this thesis.

A first goal of this thesis is thus to understand how abyssal mixing layers couple with the large-scale circulation to support a finite diabatic overturning at equilibrium. A second goal is to clarify the processes that maintain a sufficiently strong near bottom stratification to support substantial upwelling in the BBL. A third goal is to extend ideas from large-scale models and sloping boundary layer theory to account for the characteristic topographic roughness of mid-ocean ridges (both abyssal hills and fracture zone canyons). An overarching fourth goal is to unify the three largely independent approaches to the abyssal mixing problem discussed above: 1) global watermass transformation diagnostics, 2) in-situ observations of mixing, stratification, currents, and tracer transport along the Mid-Atlantic Ridge in the Brazil Basin, and 3) sloping boundary layer theory. As described above, several of these past approaches to mixing-driven abyssal upwelling are (at least superficially) in direct conflict (e.g. sloping boundary layer flows appear too weak to sustain the observed near-bottom stratification and the inferred watermass transformations), hindering the development of a consensus view and slowing progress.

The problem is perhaps best illustrated by marrying two of Walter Munk's analogies Munk, 1966, Munk and Wunsch, 1998): most approaches to the abyssal mixing problem are like trying to bake an apple pie using oranges, apple-sauce, or an generic spherical fruit; below, I present progress towards a more coherent and comprehensive abyssal recipe.

\subsection{The thesis statement}

Together, the thesis paints a revised conceptual picture of the mixing-driven abyssal overturning circulation (Figure 1-2) that is dynamically self-consistent (and at least qualitatively consistent with available observations). Dense bottom waters form at high latitudes and fill the global abyss via Deep Western Boundary Currents (DWBC) (Stommel, 1958). These bottom waters then flow adiabatically (and geostrophically) across the abyssal plains towards regions of rough topography (e.g. mid-ocean ridges), where bottom-enhanced mixing causes buoyancy to converge within a thin bottom boundary layer, driving diapycnal upwelling of $\mathcal{E}_{\mathrm{BBL}}=\mathcal{O}(20$ to $50 \mathrm{~Sv})$. However, this diapycnal upwelling is partially offset by an interior downwelling of $\mathcal{E}_{\mathrm{SML}}=-\mathcal{O}(10$ to $40 \mathrm{~Sv})$, 
resulting in a net overturning of $\mathcal{E}=\mathcal{E}_{\mathrm{BBL}}+\mathcal{E}_{\mathrm{SML}}=\mathcal{O}(10$ to $30 \mathrm{~Sv})$. The average water parcel experiences this net diabatic upwelling as it transforms from Bottom Water into lighter Deep Water. The newly formed Deep Water then flows adiabatically back towards the west, where a frictional DWBC allows a return flow towards the Southern Ocean9. In the Antarctic Circumpolar Current, a combination of winds and mesoscale eddies upwell the deep water adiabatically to the surface along sloping isopycnals (Wolfe and Cessi, 2011; Marshall and Speer, 2012), at which point surface buoyancy fluxes close the abyssal loop by transforming the deep waters back into bottom waters (or lightening the waters and connecting the abyssal cell to the North Atlantic Deep Water cell of the overturning, as described by Talley 2013a and Tamsitt et al. 2018).

The magnitude of BBL upwelling $\mathcal{E}_{\mathrm{BBL}}$ is set by the minimum of the vertical buoyancy flux profile $F_{(z)} \approx-\kappa b_{z}$, or, for a fixed bottom-enhanced diffusivity profile, the vertical structure of the strat ification within a few hundred meters of the boundary where diffusivities are large. Mixing tends to homogenize mixing layers but also produces buoyancy forces that drive a "secondary" up-slope circulation; while sloping boundary layer theory predicts that this cross-slope advection maintains a non-zero stratification at equilibrium, the corresponding watermass transformations are too small to contribute significantly to the abyssal overturning circulation. Three-dimensional submesoscale baroclinic eddies and topographic steering/blocking effects, however, conspire to maintain a suffi ciently large stratification of $b_{z}=\mathcal{O}\left(10^{-7} \mathrm{~s}^{-2}\right)$ to support the inferred $\mathcal{E}_{\mathrm{BBL}}$. The net overturning $\mathcal{E}$, on the other hand, depends largely on the degree of compensation by diabatic downwelling $\mathcal{E}_{\mathrm{SML}}$ in the SML above. Since the SML's buoyancy flux divergence is spread over a much thicker $\mathcal{O}(1000 \mathrm{~m})$ outer layer and is thus locally weak, it is more susceptible to being modified by coupling with the large-scale circulation. In particular, increases in the basin-wide stratification with height (or buoy ancy) significantly reduce the buoyancy flux divergence in the SML (relative to that predicted by sloping boundary layer theory), allowing a finite net overturning $\mathcal{E}$ at all depths.

In each Chapter, I compare my models against available observations. The simulated basinscale overturning driven by bottom-enhanced mixing along a rough mid-ocean ridge is qualitatively consistent with available diagnostic estimates for similarly-sized regions in each ocean basin (Chapter 2. Figure 2-12). Similarly, the simulated overturning circulation in a small sub-region of the Brazil Basin is quantitatively consistent (within a factor of 2) with observations of the fracture zone canyon's stratification, up-canyon mean flows, and the diapycnal transport and diapycnal spreading

\footnotetext{
${ }^{9}$ Of course, such watermass transformations also occur within DWBCs and the Southern Ocean as long as there is substantial mixing, as has been observed in a Southern Ocean DWBC just downstream of a Bottom Water formation site (Garabato et al., 2019, Spingys et al. 2021).
} 
of a purposefully released inert chemical tracer (Chapters 3 and 4). My theoretical and simulation results from Chapter 4 suggest that observations of the tracer-weighted buoyancy tendency from the on-going Bottom Boundary Layer Turbulence and Abyssal Recipes field campaign should provide a reasonable estimate of the buoyancy velocity; a large positive value would be strong supporting evidence for the ideas in this thesit10

\subsection{Structure of the thesis}

\subsubsection{Chapter 2; basin-scale dynamics}

In Chapter 2, I use Callies and Ferrari (2018b)'s Planetary Geostrophic Circulation Model (PGCM) to configure the simplest possible model of a mixing-driven basin-scale abyssal overturning cir culation. To capture the leading order spatial patterns of deep ocean mixing (e.g. the famous Brazil Basin zonal section from Polzin et al. 1997), I impose a weak background diffusivity of $2.5 \times 10^{-5} \mathrm{~m}^{2} / \mathrm{s}$ in the interior and over the smooth continental slopes and abyssal plains, but im pose a strongly bottom-enhanced profile over a rough mid-ocean ridge. A simple Rayleigh drag parameterization on the horizontal velocity maintains a realistically large stratification in the mix ing layers and substantially thickens the BBL, allowing them to be resolved by the terrain-following discretization. Using a constantly-stratified initial condition, the initial spin-up of the 3D model is reasonably emulated by a mosaic of $1 \mathrm{D}$ boundary layer models. As predicted by $1 \mathrm{D}$ theory, the solution exhibits large BBL upwelling at all depths and almost-as-large SML downwelling above, such that net watermass transformations are vanishingly small except at the base of the mid-ocean ridge, where there is no SML to compensate for the convergence in $\mathrm{BBL}$.

Eventually, the imposed mixing would homogenize the whole basin; to allow a non-trivial solution at equilibrium, I add a restoring term to the buoyancy field in the Southern region of the model, a crude parameterization for the transformation of deep waters back into bottom waters in the Southern Ocean. As time evolves, initially small cross-ridge convergences drive exchanges with the interior ocean, and the basin-wide stratification begins to drift. This coupling between the interior and mixing layers results in a basin-wide equilibrium stratification that increases with height and reduces the buoyancy flux divergence in the SML, allowing a realistically large net watermass transformation over the full vertical extent of the mid-ocean ridge. Basin-wide watermass

\footnotetext{
${ }^{10}$ Rumor has it that shortly before the final submission of this thesis, the fluorescent dye released in the BBL at more than $1500 \mathrm{~m}$ did indeed rapidly upwell across buoyancy surfaces, roughly consistent with observations of vigorous turbulent mixing.
} 
Ch. 2: Coupling of basin-scale overturning to bottom boundary layers (BBLs)

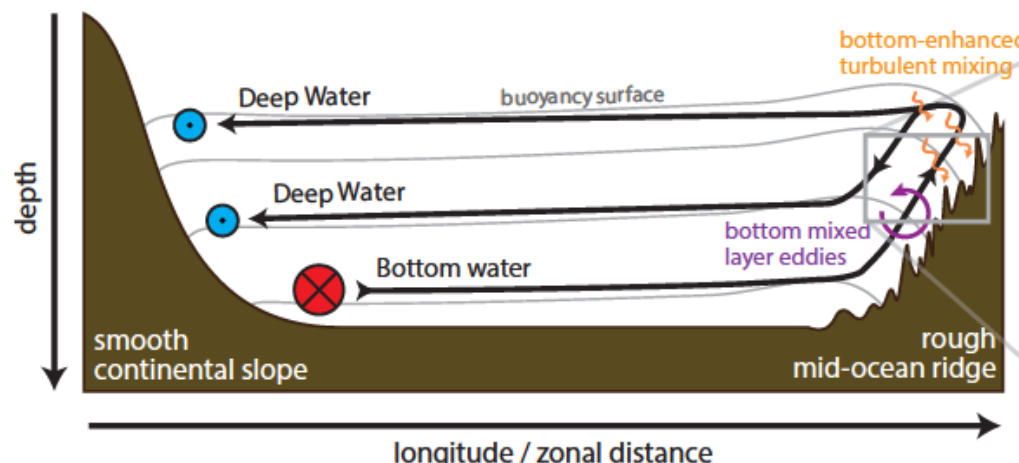

longitude / zonal distance
Ch. 3: Eddying BBLs over rough topography

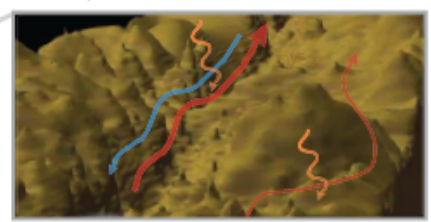

Ch. 4: Tracer transport in BBLs

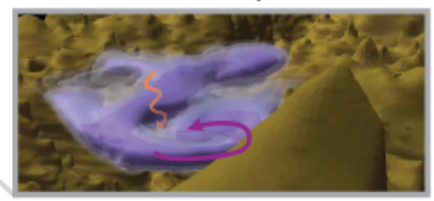

Figure 1-2

transformations are dominated by the flux divergences in the relatively thin mixing layers on the ridge. Indeed, the local correlations between the diffusivity and the stratification are at least as large as the product of their respective horizontal averages (or averages along a density surface), which typically enter in the prognostic equations of one-dimensional (Munk and Wunsch, 1998) or zonally-integrated (Nikurashin et al., 2012) models of the abyssal circulation.

\subsubsection{Chapters 3 and 4: regional dynamics and tracer transport}

A major limitation of Chapter 2 is the relatively arbitrary choice of a Rayleigh drag parameterization as a stand-in for the poorly understood sub-gridscale physical processes that maintain the stratification of abyssal mixing layers. In Chapters 3 and 4 , I instead zoom in on a specific region, centered around a well-observed fracture canyon on the western flank of the Mid-Atlantic Ridge in the Brazil Basin. This regional scope allows us to run higher-resolution simulations that resolve key restratification processes, with the trade-off being that we lose the two-way coupling to the basin-scale circulation. Inspired by sloping boundary layer theory, I develop a novel configuration of the MIT General Circulation Model in which I solve for the perturbations relative to a constantlystratified background state and then rotate the perturbation equations to be aligned with a mean topographic slop $\AA^{11}$. This formulation allows us to apply doubly-periodic boundary conditions along the plane of the mean slope and provides a natural restratification process by cross-slope advection across a background vertical stratification (projected onto the cross-slope direction). The regional simulations resolve the characteristic abyssal hills and fracture zone canyons of the mid-ocean ridge, as well as the submesoscale instabilities expected based on idealized linear stability calculations.

\footnotetext{
${ }^{11}$ Callies (2018) configures a similar model using the Dedalus code (Burns et al., 2016), but does not allow for any topographic variations along the plane of the slope.
} 
In Chapter 3, I describe the structure of the mixing-driven cross-slope mean flow, its strat ification, and its finite-amplitude instabilities. Both watermass transformations analysis and a generalized slope-normal buoyancy budget reveal that the maintenance of a strong near-bottom stratification allows efficient mixing to drive much larger BBL upwelling than implied by 1D bound ary layer dynamics (both within the fracture zone canyon and on the surrounding ridge flanks). Using a series of mechanism denial experiments in a hierarchy of progressively idealized simula tions, I identify the three leading-order restratification processes: 1) slumping of isopycnals by finite-amplitude submesoscale baroclinic instabilities (Wenegrat et al., 2018; Callies, 2018); 2) the blocking of rotational effects within narrow fracture zone canyons (Thurnherr and Speer, 2003 , Dell, 2013; Ruan and Callies, 2020); and 3) the effect of sills in blocking up-canyon mean flows and homogenizing depressions below the sill height (Winters and Armi, 2012, Dell, 2013).

In Chapter 4, I use this quasi-realistic regional model configuration to simulate the Brazil Basin Tracer Release Experiment (BBTRE), in which an inert chemical tracer was injected in the SML and its evolution was traced over several years. I apply a recently developed tracer-weighted buoy ancy moment approach (Ruan and Ferrari, 2021) to exactly attribute the mean diapycnal transport and diapycnal spreading of the tracers to the underlying mixing-driven diabatic forces. A surprising result is that non-linear diapycnal stretching effects in this experiment are not important for interior releases due to almost perfect compensation by stretching in the SML and contraction by boundary suppression (such as in the BBTRE), but can be as large as the straightforward diapycnal diffusion effect for near-bottom releases. Transiently, I find that diapycnal diffusion of a tracer can counter intuively cause net diapycnally contraction. I show that the simulated downwelling and diapycnal spreading of the BBTRE tracer is quantitatively consistent with the tracer evolution observed by Ledwell et al. (2000). I also simulate a tracer released directly in the BBL and another released roughly at the height of the crests of the canyon flanks, roughly at the expected transitional bound ary between buoyancy convergence (diabatic upwelling) and divergence (diabatic downwelling). The tracer-weighted moments of the three tracers (in principle observable) provide reasonable quantita tive estimates of four key mixing layer quantities: the upwelling buoyancy velocity in the BBL, the downwelling buoyancy velocity in the SML, and the in-situ height-above-bottom-averaged diffusivity profile (except in the BBL where diapycnal stretching dominates). 


\section{Chapter 2}

\section{Abyssal circulation driven by near-boundary mixing: water mass transformations and interior stratification}

This work is now published as Drake et al. (2020a) in the Journal of Physical Oceanography, (C) American Meteorological Society. Used with permission.

\subsection{Motivation}

The abyssal ocean, below $2500 \mathrm{~m}$, is a massive reservoir for climatically active tracers such as carbon and heat. The rates at which heat is mixed and advected into the high capacity abyssal ocean are key parameters in understanding both past climate reconstructions (e.g. Toggweiler et al., 1989) and future projections of climate change (e.g. Hansen et al., 1985). Similarly, the partitioning of carbon between the deep ocean and the atmosphere is a major factor on millennial-scale climate change, whether natural (e.g. Sarmiento and Toggweiler, 1984) or anthropogenic in origin (Archer et al. 1998). It is thus vital to have a firm phenomenological and dynamical understanding of the abyssal ocean's mean state.

The general structure of the abyssal ocean circulation is easily inferred from surface buoyancy fluxes and large-scale tracer properties (Sverdrup et al., 1942). Antarctic Bottom Waters, the densest oceanic waters, form in the Southern Ocean and fill the global abyssal oceans up to a depth of about $2500 \mathrm{~m}$ (Talley, 2013a). They outcrop at the surface only in the Southern Ocean, where they experience a significant area-integrated buoyancy loss (Abernathey et al., 2016) and are converted back into lighter waters by mixing with lighter overlying waters, resulting in a diabatic abyssal 
overturning circulation of $O(15 \mathrm{~Sv})$, where $1 \mathrm{~Sv}=10^{6} \mathrm{~m}^{3} \mathrm{~s}^{-1}$. Non-linearities in the equation of state of seawater and geothermal heating at the seafloor are thought to play secondary roles in shaping this circulation (Emile-Geay and Madec, 2009; de Lavergne et al., 2016b) and will be ignored in the conceptual models described below.

Classical theories for the abyssal ocean describe the steady state circulation and stratification of a flat-bottom ocean forced by uniform turbulent mixing (Stommel, 1957; Robinson and Stommel, 1959, Stommel and Arons, 1959b a; Munk, 1966). These theories remain pedagogically useful, but are at best qualitative descriptions, as demonstrated for example by the fact that the direction of the flow in the Stommel and Arons (1959b) solution changes sign when a sloping seafloor is introduced (Rhines, 1993) and that the Munk (1966) solution does not satisfy the no-flux boundary condition at the seafloor. The classical view of a uniform mixing-driven upwelling is further challenged by the observation that turbulent mixing is typically bottom-enhanced over rough topography (see MacKinnon et al. 2017 for a recent review), reversing the sign of the vertical flow implied by the interior ocean vertical density balance (Polzin et al., 1997; Ferrari et al., 2016).

Since Munk (1966), several approaches have been taken to address the limitations of classical theories. First, boundary layer theories (Wunsch, 1970; Thorpe, 1987; Garrett, 1990; Thompson and Johnson, 1996) arose to elucidate the local behavior of mixing-induced flow along a sloping and insulating sea floor. Second, the limitations of the Stommel and Arons $(1959 \mathrm{~b})$ theory in spired a number of extensions to account for baroclinic structure (Kawase, 1987; Pedlosky, 1992), non-uniform seafloor depth (Rhines, 1993), and/or non-uniform turbulent diffusivities $\kappa$ (Marotzke, 1997; Samelson, 1998). Third, the observation of bottom-enhanced mixing motivated the devel opment of progressively more sophisticated parameterizations of vertical (or diapycnal) turbulent diffusivities (Bryan and Lewis, 1979; St. Laurent and Garrett, 2002, Polzin, 2009; Lavergne et al., 2020 which have been subsequently implemented into general circulation models Huang and Jin, 2002, Jayne, 2009, Melet et al. 2016; Boucher et al., 2020). Fourth, the conundrum of interior downwelling implied by bottom-enhanced mixing was resolved by applying the watermass transformation framework to a downwelling interior layer of turbulent buoyancy flux divergence and an upwelling bottom boundary layer of turbulent buoyancy flux convergence, respectively (Ferrari et al., 2016, de Lavergne et al., 2016c; McDougall and Ferrari, 2017). Despite the direct relevance of all of these approaches to the abyssal circulation 1 , there has been little work done to unify them into a general

\footnotetext{
${ }^{1}$ Regional heat budgets and inverse models have also been used to estimate mixing rates and overturning circula tions from available observations (Hogg et al. 1982, Ledwell et al., 2000, St. Laurent et al., 2001, Morris et al. 2001), and deserving mentioning. While we do not engage much with this ideas in this Chapter, we revisit them in detail in
} 
theory of the abyssal circulation and stratifcation.

Building on the framework introduced by Callies and Ferrari (2018b) (hereafter, CF18), we present a unified prognostic model of the circulation in an abyssal basin forced by bottom-enhanced mixing along a mid-ocean ridge. We modify the geometry, buoyancy forcing, and initial condition of the CF18 model to include the effects of a smooth mid-ocean ridge (with the effects of local roughness parameterized by bottom-enhanced mixing) and of a non-uniform background stratification on the circulation. Our approach is to formulate the simplest possible model which captures what we believe to be the key aspects of the problem: 1) the transformation of abyssal bottom waters into relatively lighter deep waters by bottom-enhanced mixing on the flanks of a mid-ocean ridge, 2) frictional processes acting on boundary currents, 3) restratification of abyssal mixing layers by baroclinic turbulence (crudely parameterized as a linear drag on the horizontal flow), and 4) bottom water formation in the Southern Ocean.

The general structure of the abyssal circulation that emerges from the model consists of layered deep western boundary currents along the western continental slope which are connected by zonal flows to watermass transformations driven by bottom-enhanced mixing along a mid-ocean ridge, as schematized in Figure 2-1. The evolution of the interior stratification and the mixing layer watermass transformations are coupled by slope-normal exchange flows, with the vertically-varying equilibrium stratification being determined by a combination of the mixing layer dynamics and the formation of dense waters in the south. Finite net watermass transformations arise ubiquitously along the flanks of the mid-ocean ridge, supported by vertical variations in the interior stratification, such that the crest of the mid-ocean ridge determines the vertical extent of the abyssal overturning cell, in contrast to a previous constant-stratification interpretation in which finite net transformations are confined to the base of topographic slopes (CF18).

The paper is structured as follows. Section 2.2 reviews the results of several theories of abyssal stratification and circulation in the literature. Section 2.3 presents the formulation of the Planetary Geostrophic Circulation Model (PGCM) used to produce the simulation results presented in the paper. Section 2.4 describes the general structure of the abyssal circulation as it emerges in the PGCM. In Section 2.5 we use local solutions to the one-dimensional boundary layer equations to emulate the three-dimensional abyssal circulation in the PGCM. Section 2.6 describes the spin-up to equilibrium of the vertical structure of abyssal interior stratification and its influence on watermass transformations. Section 2.7 compares watermass transformations in our PGCM simulations with Chapters 3 and 4 


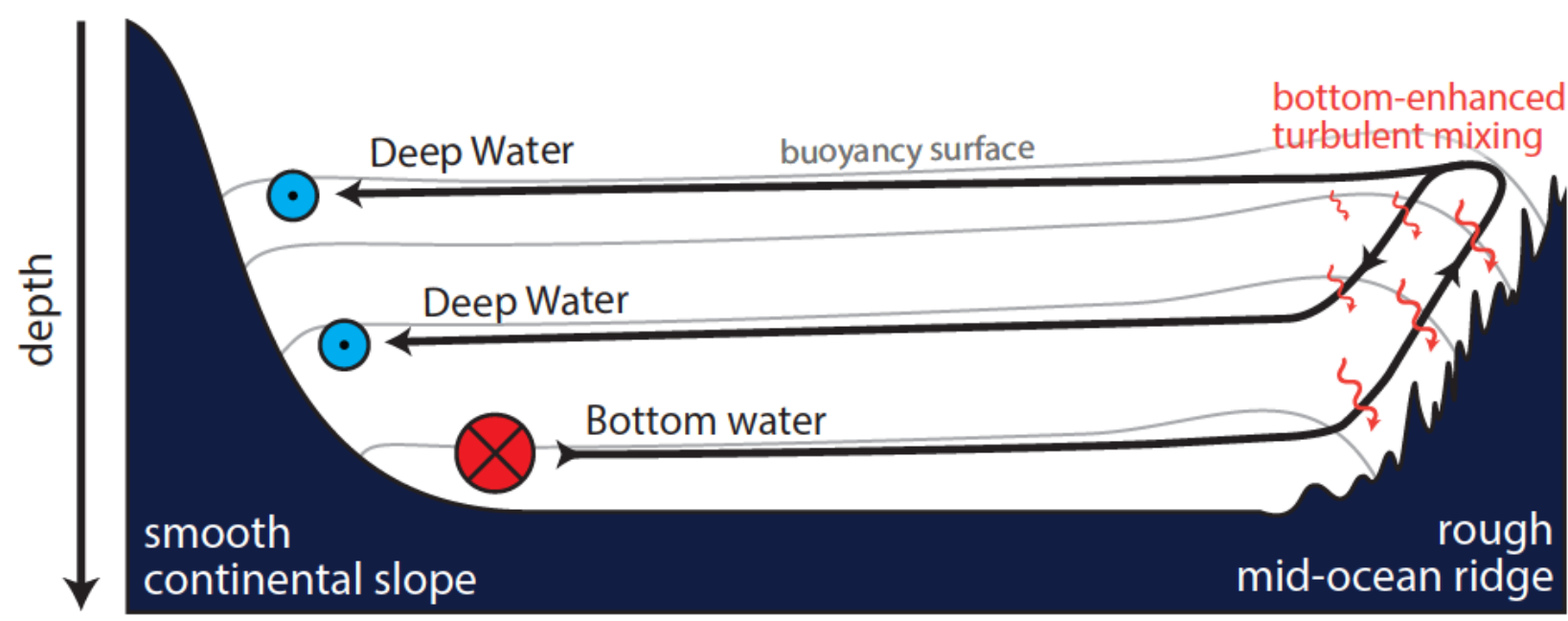

longitude / zonal distance

Figure 2-1: Schematic of a basin-scale abyssal circulation driven by near-boundary mixing. Dense bottom waters flow northward out of the Southern Ocean via a deep western boundary current (red circle) along the smooth and relatively quiescent continental slope, where little watermass transformation occurs. A cross-basin zonal flow feeds bottom waters from the deep western boundary current into a system of abyssal mixing layers driven by bottom-enhanced turbulent mixing over the rough topography of the mid-ocean ridge flanks (squiggly orange arrows). The turbulent buoyancy flux converges in a bottom boundary layer (BBL), driving vigorous diabatic upwelling across buoyancy surfaces (grey lines). In a stratified mixing layer (SML) above, the buoyancy flux diverges, driving diabatic downwelling. The net effect of the up- and down-welling in the abyssal mixing layers is a net transformation of bottom waters into deep waters. The newly formed deep waters return via cross-basin zonal flows to the smooth continental slope, wherein they flow southward in a deep western boundary current (blue circles) to close the abyssal circulation in the Southern Ocean. For simplicity, we omit the alternating along-ridge flows (see Figure 2-3b) that are in frictional thermal-wind balance with the plunging isopycnals.

estimates for the mid-ocean ridges of the Pacific, Atlantic, and Indian Ocean basins. Section 2.8 compares diagnostic estimates of abyssal upwelling from the watermass transformation framework with the classic vertical advection-diffusion framework and evaluates the relative contributions of various physical terms of the watermass transformation. Section 2.9 discusses the implications of our results, some key caveats, and some promising future directions. 


\subsection{Theoretical Background}

\subsubsection{Classical theories of abyssal stratification and circulation}

Modern theories of the abyssal circulation begin with a series of papers by Stommel and Arons (1959b a). In their theory, the circulation of a homogeneous abyssal layer is fed by high-latitude sources of abyssal water (diabatic downwelling) and driven by a uniformly-distributed sink (diabatic upwelling) of abyssal water. A uniform upwelling across the base of the thermocline is prescribed, inspired by the thermocline-thermohaline theory of Robinson and Stommel (1959). Munk (1966) further simplifies the Robinson and Stommel (1959) balance by restricting his attention to the deep ocean (i.e. below the thermocline) and by considering only vertical advection and diffusion,

$$
u^{z} \partial_{z} b=\partial_{z}\left(\kappa \partial_{z} b\right)
$$

where $b$ is buoyancy, $u^{z}$ is a uniform vertical velocity, $\kappa$ is a uniform turbulent diffusivity. The Munk formulation allows exponential solutions which can be fit to the observed temperature profiles and combined with fits of an advection-diffusion-decay equation to radiocarbon profiles to yield the canonical estimate of deep ocean mixing $\kappa \simeq 10^{-4} \mathrm{~m}^{2} \mathrm{~s}^{-1}$ for a uniform upwelling of $u^{z}=1.4 \times 10^{-7}$ $\mathrm{m} / \mathrm{s}$.

The horizontal abyssal circulation associated with the upwelling is described by Stommel and Arons (1959b a): interior flow is geostrophically-balanced and its meridional component $u^{y}$ is driven by vortex stretching, as shown by the vertically-integrated planetary-geostrophic vorticity balance

$$
\beta U^{y}=f \frac{u_{0}^{z}}{H}
$$

where $H$ is the thickness of the abyssal layer, $u_{0}^{z}>0$ is the upwelling across the base of the thermocline, $f$ is the Coriolis parameter, $\beta>0$ is the meridional gradient of the Coriolis parameter, and the vertically-integrated flow $U^{y}$ is thus poleward in both hemispheres (see Pedlosky 1996 for an elucidating derivation). Inspired by the success of analogous theories for the wind-driven gyre circulation (Stommel, 1948), Stommel and Arons (1959b a) suppose the existence of a deep western boundary current in which frictional effects allow the current to deviate from geostrophy and return the interior flow such that the abyss conserves mass. 


\subsubsection{Turning ocean mixing upside down}

The Stommel and Arons (1959ba) and Munk (1966) theories rely on the existence of a uniform turbulent diffusivity $\kappa \simeq 10^{-4} \mathrm{~m}^{2} \mathrm{~s}^{-1}$, roughly an order of magnitude larger than the interior ocean mixing inferred from observations (Gregg, 1989; Ledwell et al., 1993). While sufficiently vigorous mixing was eventually discovered deeper in the ocean near rough seafloor topography (Polzin et al., 1997, Ledwell et al., 2000, Sheen et al. 2013), the abyssal mixing problem only became more complicated: applying the vertical advection-diffusion balance (eq. 2.1) point-wise to mixing profiles $\kappa(z) \partial_{z} b$ that increase with depth implies diapycnal downwelling

$$
u^{z}=\left(\partial_{z} b\right)^{-1} \partial_{z}\left(\kappa \partial_{z} b\right)<0
$$

in contrast to the diapycnal upwelling required to balance diapycnal downwelling at high latitudes ${ }^{2}$.

This apparent conundrum is resolved by considering the insulating boundary condition at a sloping seafloor, which causes buoyancy convergence and hence diapycnal upwelling in a thin bottom boundary layer (Polzin et al., 1997; Ferrari et al., 2016; de Lavergne et al., 2016c). In this framework, the abyssal overturning is the net effect of downwelling driven by bottom-enhanced mixing in a stratified mixing layer and upwelling driven by buoyancy convergence in a bottom boundary layer, which we collectively refer to as abyssal mixing layers (CF18).

\subsubsection{A puzzling constraint from boundary layer theory}

Bottom boundary layer theory (see review of Garrett et al., 1993) is a useful dynamical approach to the problem of flow driven by near-boundary mixing on a slope, which exerts a strong control on the basin-scale abyssal circulation (CF18). Following Thorpe (1987), who built on the approaches of Wunsch (1970) and Phillips (1970), we rotate the Boussinesq equations into slope coordinates and assume the flow depends only on the slope-normal coordinate $z^{\prime}$, which gives the simplified buoyancy equation (see derivation of full equation set in Section 2.5.1):

$$
\partial_{t} b^{\prime}+u^{x^{\prime}} N_{0}^{2} \sin \theta=\partial_{z^{\prime}}\left[\kappa\left(N_{0}^{2} \cos \theta+\partial_{z^{\prime}} b^{\prime}\right)\right]
$$

where $u^{x^{\prime}}$ is the up-slope velocity, $\theta$ the slope angle, $\kappa=\kappa\left(z^{\prime}\right)$ the turbulent diffusivity, and we decompose the buoyancy field $b(x, y, z, t)=N_{0}^{2} z+b^{\prime}(x, y, z, t)$ into a background corresponding to a

\footnotetext{
${ }^{2}$ While the sign of the vertical velocity changes, we note that $\partial_{z} u^{z}>0$ and thus the interior geostrophic flow driven by vortex stretching is still of the same sign as in the Stommel-Arons solution.
} 
constant stratification $N_{0}^{2}$ and a buoyancy anomaly $b^{\prime}=b^{\prime}\left(z^{\prime}\right)$. The boundary conditions are a no flux condition $\partial_{z^{\prime}} b=\partial_{z^{\prime}} b^{\prime}+N_{0}^{2} \cos \theta=0$ at the seafloor $z^{\prime}=0$ and decay conditions $\partial_{z^{\prime}} u^{x^{\prime}}, \partial_{z^{\prime}} b^{\prime} \rightarrow 0$ as $z^{\prime} \rightarrow \infty$. At steady state, the boundary layer equation for the buoyancy anomaly (eq. 2.4) can be integrated from $z^{\prime}=0$ to $z^{\prime} \rightarrow \infty$, which yields an integral constraint

$$
\Psi_{\mathrm{bg}} \stackrel{\text { def }}{\equiv} \kappa_{\mathrm{bg}} \cot \theta
$$

for the net up-slope transport per unit length $\Psi_{\text {bg }}=\int_{0}^{\infty} u^{x^{\prime}} \mathrm{d} z^{\prime}$, where $\kappa_{\text {bg }} \stackrel{\text { def }}{\equiv} \kappa(z \rightarrow \infty)$ is the background diffusivity. The simplicity of this integral constraint is surprising: the net up-slope transport depends only on the background turbulent diffusivity $\kappa_{\mathrm{bg}}$ and the slope angle $\theta$, and is independent of other environmental parameters which might be expected to influence diapycnal transport, such as frictional parameters, the background stratification $N_{0}^{2}$, the Coriolis parameter $f$, and the vertical structure of the turbulent diffusivity $\kappa(z)$.

Integrating the prediction $\Psi_{\mathrm{bg}}$ for the diapycnal transport per unit length along the perimeter $L_{\text {global }} \simeq 10^{8} \mathrm{~m}$ of the global mid-ocean ridge system (Callies, 2018) for a typical ridge slope $\tan (\theta)=2 \times 10^{-3}$ and a background diffusivity of $\kappa_{\mathrm{bg}} \simeq 10^{-5} \mathrm{~m}^{2} \mathrm{~s}^{-1}$ produces a global mixingdriven diapycnal overturning transport of $L_{\text {global }} \kappa_{\mathrm{bg}} \cot \theta \simeq 0.5 \mathrm{~Sv}$, more than an order of magnitude smaller than the inferred abyssal diapycnal overturning transport of roughly $15 \mathrm{~Sv}$ (Lumpkin and Speer, 2007).

CF18 resolve this conundrum by using the magnitude of the upwelling-downwelling 'dipole' from boundary layer theory as a prediction for the net watermass transformation, since at the base of topographic slopes the flows in and out of the boundary layers occur at different density classes and thus drive a diabatic overturning. They find that the strictly upwelling transport in the bottom boundary layer accurately predicts the scaling of the maximum net diapycnal overturning transport, although the predicted overturning is unrealistically confined to the base of topographic slopes, where the constraints from one-dimensional boundary layer theory break down.

\subsubsection{Boundary-interior exchange}

The integral constraint $\Psi_{\mathrm{bg}} \equiv \kappa_{\mathrm{bg}} \cot \theta$ (eq. 2.5) relies on the assumption of constant background stratification $N_{0}^{2}$ and slope angle $\theta$. By construction, none of the other terms are assumed to vary in the plane of the slope $\left(x^{\prime}, y^{\prime}\right)$ either; it follows that there are no cross-slope convergences $\partial_{x^{\prime}} u^{x^{\prime}}=0$ and hence no slope-normal exchange between the abyssal mixing layers and the interior, $u^{z^{\prime}}=0$ 
(Wunsch, 1970).

With a vertically varying stratification $N^{2}(z)$, however, variations in the buoyancy gradient project onto the cross-slope direction $x^{\prime}=x \cos \theta+z \sin \theta$, introducing a second dimension to the problem (e.g. Phillips et al., 1986; Salmun et al., 1991) and permitting both slope-normal exchange

flows $u^{z^{\prime}} \neq 0$ and a net diapycnal transport $\Psi_{\infty} \stackrel{\text { def }}{=} \int_{0}^{\infty} u^{x^{\prime}} \mathrm{d} z^{\prime} \neq \Psi_{\mathrm{bg}}$. Heterogeneities can also arise due to cross-slope variations in the turbulent diffusivity $\kappa(x, y)$ or the slope angle $\theta(x, y)$ (Dell and Pratt, 2015), and have been argued to contribute significantly to oceanic watermass transformations (McDougall and Ferrari, 2017; de Lavergne et al., 2017; Holmes et al., 2018). These additional heterogeneities are both kept relatively small by construction in our idealized model configuration to keep the focus on the effects of variations in the basin stratification.

\subsubsection{Dynamics controlling the interior abyssal stratification}

The abyssal stratification is thought to be controlled by the combined effects of 1) diapycnal mixing in ocean basins and 2) the competing effects of winds and mesoscale eddies in setting the slope of isopycnals in the Southern Ocean. Diapycnal mixing maintains the stable stratification of the abyssal ocean by effectively diffusing buoyancy downwards, transforming dense abyssal waters into lighter deep waters (Munk, 1966). This vertical advection-diffusion model is an incomplete model of the abyssal stratification, however, as it omits the complementary process which closes the overturning circulation by transforming light deep waters into denser abyssal waters. Munk and Wunsch (1998) consider a heuristic correction to Munk (1966)'s vertical advection-diffusion equation for the effect of horizontal advection from regions of high mixing (or homogenization by convection), which acts to restratify regions of weak mixing. A breakthrough in understanding the abyssal stratification was the development of quasi-adiabatic theories of Southern Ocean circulation. In these theories, deep waters are upwelled adiabatically along sloping isopycnals in the Southern Ocean, are transformed into abyssal waters in the Southern Ocean mixed layer by a negative surface buoyancy flux, and return to the abyss adiabatically along isopycnals (Marshall and Speer, 2012, and references therein). The Southern Ocean isopycnal slope is determined by a balance between wind stress and stirring by mesoscale eddies, which steepen and flatten isopycnals, respectively (Marshall and Radko, 2003).

Building on these two independent theories, Nikurashin and Vallis (2011) develop an idealized model which couples quasi-adiabatic Southern Ocean dynamics to a diabatic abyssal ocean basin and predicts the abyssal stratification and circulation, given only surface boundary conditions and mixing coefficients. For moderate diapycnal mixing of $10^{-5} \mathrm{~m}^{2} \mathrm{~s}^{-1}<\kappa<10^{-3} \mathrm{~m}^{2} \mathrm{~s}^{-1}$, a regime 
applicable to both the Ocean and the model described here, the Nikurashin and Vallis (2011) model predicts that the interior abyssal stratification depends both on winds and eddies in the Southern Ocean and diapycnal mixing in the basin.

A promising aspect of zonally-integrated models of the meridional overturning circulation (e.g Nikurashin et al., 2012; Thompson et al., 2016) is that they accurately reproduce the overturning and stratification exhibited by idealized "box"-geometry general circulation models. The emerging view, however, is that the abyssal circulation of the ocean is controlled by mixing layer flows along sloping boundaries and thus that the commonly-used "box" geometry models may be a misleading point of reference for theories of the abyssal stratification and circulation (Ferrari et al., 2016). Building on CF18, we describe the formulation of an improved idealized general circulation model in a "bowl + ridge" geometry which accommodates the recent revisions to our theoretical understanding of the abyssal ocean circulation.

\subsection{Planetary Geostrophic Circulation Model (PGCM)}

The numerical model used here is the Planetary Geostrophic Circulation Model (PGCM) developed by CF18 to study how bottom-enhanced mixing on slopes drives an abyssal circulation. We describe the key elements of our PGCM configuration below, which closely follows the exposition of CF18. The main differences between the present study and CF18 are the inclusion of the mid-ocean ridge, the localization of vigorous bottom-enhanced mixing to a mid-ocean ridge, and the generalization to vertically-varying interior stratifications. Readers familiar with the methods of CF18 can skip Section 2.3 and simply consult Figure 2-2, which summarizes our changes to the configuration.

\subsubsection{Equations}

The model solves the Navier-Stokes equations under the Boussinesq and planetary-scale geostrophic approximations, with parameterizations for the frictional and diabatic effects of unresolved pro cesses, given by

$$
\begin{aligned}
f \mathbf{z} \times \mathbf{u} & =-\nabla p+b \mathbf{z}-r\left(u^{x} \mathbf{x}+u^{y} \mathbf{y}\right), \\
\nabla \cdot \mathbf{u} & =0, \text { and } \\
\frac{\partial b}{\partial t}+\mathbf{u} \cdot \nabla b & =\nabla \cdot(\kappa \nabla b)-\lambda(y)(b-B(z)),
\end{aligned}
$$


where $t$ is time; $\mathbf{x}, \mathbf{y}, \mathbf{z}$ are unit vectors pointing east, north, and up, respectively; $f=\beta y$ is the linearized Coriolis parameter ( $\beta$-plane approximation $\left.{ }^{3}\right) ; \mathbf{u}=\left(u^{x}, u^{y}, u^{z}\right)$ is the velocity vector; $p$ is the pressure divided by a reference density; $b$ is the buoyancy; $r$ is a frictional parameter; $\kappa=\kappa(x, y, z)$ is a spatially-dependent turbulent diffusivity; and $\lambda=\lambda(y)$ is a meridionally-varying restoring rate (see Section 2.3.3). The system of equations (2.6) - 2.8), with appropriate initial and boundary conditions, yields a self-consistent and prognostic model of abyssal circulation and stratification.

The Boussinesq approximation filters out acoustic waves while the planetary-geostrophic approx imation filters out gravity waves and geostrophic turbulence. The resulting planetary-geostrophic equations are appropriate for basin-scale oceanic circulations and are typically used for idealized studies of the abyssal circulation (e.g Pedlosky, 1996, and references therein) and intermediatecomplexity earth system models (e.g. Holden et al., 2016). While it is computationally and con ceptually useful that the planetary-geostrophic equations filter out the effects of fast waves and turbulence, the turbulent fluxes of these relatively small-scale flows are thought to have leading or der effects on abyssal mixing layers. We include their qualitative effects in the planetary-geostrophic formulation by way of two idealized parameterizations.

First, to include the effects of turbulent mixing produced by the local breaking of internal waves generated by flow over rough topography, we introduce a term for the turbulent buoyancy flux convergence $\nabla \cdot(\kappa \nabla b)$ to the buoyancy equation (e.g. as in St. Laurent and Garrett, 2002). The imposed spatially-dependent turbulent diffusivity $\kappa(x, y, z)$ approximates the leading-order spatial structure described by observational estimates (e.g. Polzin et al., 1997; Waterhouse et al., 2014) and is described in detail in Section 2.3.3

Second, to include the qualitative effects of isopycnal mixing by baroclinic turbulence in restrat ifying the abyssal mixing layers Callies 2018 and Chapter 3) and in thickening western boundary currents (e.g. Stommel, 1948), we introduce a dissipative term to the momentum equation. Great batch and Lamb (1990) show that introducing vertical momentum diffusion $\partial_{z}\left(\nu_{\text {eddy }} \partial_{z} \mathbf{u}\right)$ to the planetary geostrophic equations with an eddy viscosity $\nu_{\text {eddy }}=\kappa_{\mathrm{GM}} f^{2} / N^{2}$ is equivalent to intro ducing isopycnal diffusion of potential vorticity with an effective isopycnal diffusivity of $\kappa_{\mathrm{GM}}$ Gent

\begin{tabular}{l}
\hline${ }^{3}$ We consider an equatorial $\beta$-plane with $f_{0}=0$ because we are interesting in a hemispherically-symmetric ocean \\
basin. \\
${ }^{4}$ Quantitatively similar profiles of turbulent kinetic energy dissipation are reproduced in simulations of internal \\
wave turbulence above rough topography, wherein energy from a geostrophic mean flow (Nikurashin and Ferrari, \\
2009) or the barotropic tide (Nikurashin and Legg, 2011$)$ is converted into unstable high-mode internal waves via a \\
cascade of wave-wave interactions.
\end{tabular}


and McWilliams, 1990). Following Salmon (1992), we simplify the dynamics further by using a linear friction term (Rayleigh drag), $-r\left(u^{x} \mathbf{x}+u^{y} \mathbf{y}\right)$ and scale the frictional parameter $r$ according to the Greatbatch and Lamb (1990) parameterization,

$$
r=\kappa_{\mathrm{GM}} \frac{f^{2}}{\delta^{2} N^{2}} \approx 1.2 \times 10^{-5} \mathrm{~s}^{-1}
$$

where we choose $\delta=400 \mathrm{~m}$ to be roughly the thickness of the abyssal mixing layers observed in the Brazil Basin (Callies, 2018); typical abyssal mixing layer values of $f=5 \times 10^{-5} \mathrm{~s}^{-1}$ and $N^{2}=5 \times 10^{-7} \mathrm{~s}^{-1}$; and assume $\kappa_{\mathrm{GM}}=100 \mathrm{~m}^{2} \mathrm{~s}^{-1}$ based on the isopycnal diffusion inferred from the Brazil Basin Tracer Release Experiment (Ledwell; in prep), which yields a value $\nu_{\text {eddy }}=0.5$ $\mathrm{m}^{2} \mathrm{~s}^{-1}$ similar to the value $\nu_{\text {eddy }}=\sigma \kappa_{\text {bot }}=0.4 \mathrm{~m}^{2} \mathrm{~s}^{-1}$ proposed by Callies (2018) and Holmes et al. (2019), where $\sigma$ is the turbulent Prandtl number. We use a constant $r$ since the parameterization is meant to be a crude placeholder for boundary layer restratification. To our relief, supplementary sensitivity experiments showed that watermass transformations and the boundary layer structure are relatively insensitive to the friction parameter $r$, in agreement with CF18. The linear drag parameter is small enough that the frictional terms are negligible in the interior where the flow is approximately geostrophic and are important only in near-boundary flows (both the deep western boundary currents and the abyssal mixing layers along the mid-ocean ridge) where the horizontal velocities are large Salmon, 1992, CF18). The choice of $r=1.2 \times 10^{-5} \mathrm{~s}^{-1}$ gives a non-dimensional value $\hat{r}=\frac{r}{\beta L}=0.1$ such that the width of the Stommel and Arons (1959ba) deep western boundary currents is one-tenth of the domain width (see Section 2.3.4).

\subsubsection{Geometry and boundary conditions}

We configure the PGCM to approximate the leading-order structure of a typical cross-hemispheric abyssal ocean basin with a rectangular basin of zonal width $L=3000 \mathrm{~km}$ and meridional length $2 L=6000 \mathrm{~km}$. Our idealized basin contains a mid-ocean ridge caused by seafloor spreading in the middle and is bounded in the west, east, and north by continental slopes (Figure 2-2 a). Although the southern region in our configuration $(y<-L / 2=-3000 \mathrm{~km})$ is also zonally bounded, it should be thought of as a Southern Ocean-like sponge layer. In this southern region, the transformation of deep waters into bottom waters arising from complex circumpolar channel dynamics (e.g. as described in Marshall and Speer, 2012) are parameterized by an idealized buoyancy restoring forcing which pins the buoyancy field to a reference vertical profile (described in detail in the next Section 2.3.3). The 
model extends from $z=-2500 \mathrm{~m}$ at the upper boundary to a maximum depth of $z=-5000 \mathrm{~m}$ and should be interpreted as representing only the diabatic lower cell of the meridional overturning circulation. The idealized configuration can be thought to apply locally to the Atlantic, Pacific, and Indian Ocean basins below $z=-2500 \mathrm{~m}$, which in the present climate are all bounded by topography in the west, east, and north and have roughly meridionally-aligned mid-ocean ridges (e.g. those highlighted in Figure 2-12). The idealized continental slopes are half-Gaussian and the mid-ocean ridge is Gaussian in the zonal direction and tapers down to zero meridionally in the southern restoring region to allow unconstrained zonal flows to close the circulation of interest in the diffusively-forced basin to the north. The characteristic seafloor slopes of roughly $\tan \left(\theta_{\text {ridge }}\right) \simeq$ $2 \times 10^{-3}$ for the mid-ocean ridge and $\tan \left(\theta_{\text {cont. }}\right) \simeq 4 \times 10^{-3}$ for the continental slope are inspired by the South Atlantic, where the abyssal mixing layers and large-scale abyssal circulation are best constrained by existing observations (Hogg et al., 1982; Polzin et al., 1997; Ledwell et al., 2000, St. Laurent et al., 2001; Thurnherr et al., 2005). The PGCM is bounded from above by assuming isopycnals are flat, i.e. $b=0$ at $z=-2500 \mathrm{~m}$, which is approximately valid in all basins north of the Southern Ocean (Talley, 2007, Koltermann et al., 2011; Talley, 2013b). The PGCM is bounded from below by an insulating seafloor, $\mathbf{n} \cdot \nabla b=0$ at $z=-d(x, y)$, where $d(x, y)$ is the seafloor depth and $\mathbf{n}$ is the unit vector normal to the boundary.

\subsubsection{Buoyancy forcing}

The abyssal circulation in our model is forced by two competing diabatic terms in the buoyancy equation: minus the divergence of the turbulent buoyancy flux $-\nabla \cdot(-\kappa \nabla b)$, which has a positive integral contribution (diapycnal upwelling); and restoring to a reference buoyancy profile $-\lambda(b-B)$, which must necessarily have a negative integral contribution (diapycnal downwelling). Available potential energy is produced by parameterized turbulent mixing and converted into kinetic energy via the buoyancy production term $u^{z} b$ to drive a planetary-geostrophic abyssal circulation and balance the available potential energy loss due to restoring.

\section{Turbulent mixing}

The prescribed turbulent diffusivity $\kappa=\kappa(x, y, z)$ is everywhere bottom-enhanced with a contri bution equal to $\kappa_{\text {bot }} \exp \{-(z+d) / h\}$ over the mid-ocean ridge, where we choose $\kappa_{\text {bot }}=5 \times 10^{-3}$ $\mathrm{m}^{2} \mathrm{~s}^{-1}$ and $h=250 \mathrm{~m}$ to roughly match observations in the Brazil Basin (Figure 2-11). The bottom-enhanced contribution to $\kappa$ is reduced by a factor of 20 to $\frac{\kappa_{\text {bot }}}{20} \exp \{-(z+d) / h\}$ over the 
continental slopes to reflect the observed weakness of local wave-driven turbulence over smooth continental slopes (Figure 2-11 and Polzin et al. 1997). A uniform weak background diffusivity $\kappa_{\text {bg }}=\frac{\kappa_{\text {bot }}}{200}=2.5 \times 10^{-5} \mathrm{~m}^{2} \mathrm{~s}^{-1}$ is added to stabilize the numerical solution, yielding a total diffu sivity distribution

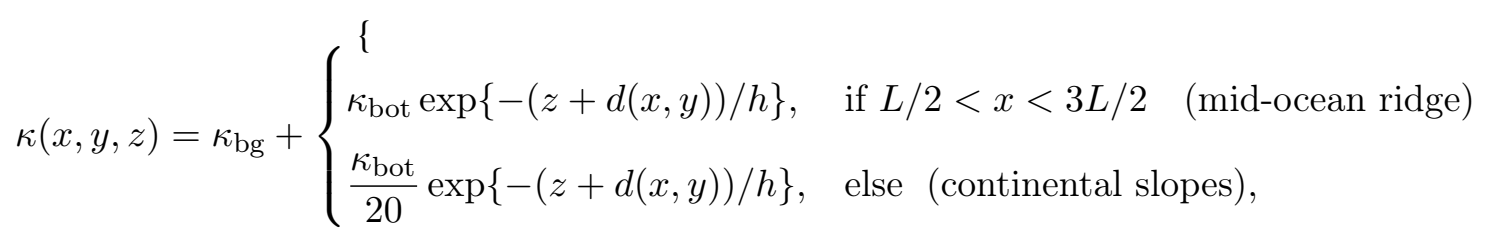

with a smoothing function applied over a horizontal distance of $L / 10$ near the transitions at $x=L / 2$ and $x=3 L / 2$. The net effect of this prescribed mixing is to power a diabatic upwelling along the mid-ocean ridge, where mixing is vigorous.

\section{Buoyancy restoring in the southern restoring region}

The prescribed restoring rate $\lambda$ has a meridional dependence

$$
\lambda(y)=\lambda_{0}\left[0.5\left(1-\tanh \left(\frac{\hat{y}+L / 2}{10 L}\right)\right)\right]^{\prime},
$$

which is equal to $\lambda_{0} \simeq(10 \text { years })^{-1}$ in the southern restoring region and vanishes rapidly northwards, $\lambda \rightarrow 0$ as $y>-L / 2$. The prescribed restoring rate is chosen based on the baroclinic adjustment timescale given by a lateral diffusive timescale $\tau_{S O}=L_{S O}^{2} / \kappa_{G M}=\frac{\left(10^{6} \mathrm{~m}\right)^{2}}{3000 \mathrm{~m}^{2} \mathrm{~s}^{-1}} \simeq 10$ years, determined for an isopycnal diffusivity $\kappa_{G M} \simeq 3000 \mathrm{~m}^{2} \mathrm{~s}^{-1}$ Abernathey et al. 2013) and a Southern Ocean cir cumpolar current of meridional width $L_{S O} \simeq 1000 \mathrm{~km}$. This restoring rate is much faster than the vertical diffusive timescale which spins up the overturning circulation $\tau_{\text {mix }}=H^{2} / \bar{\kappa} \simeq 1000$ years, where $H=2500 \mathrm{~m}$ is the maximum thickness of the abyssal ocean and $\bar{\kappa} \simeq 10^{-4} \mathrm{~m}^{2} \mathrm{~s}^{-1}$ is the volume-weighted mean diffusivity in the basin. Thus, the stratification in the southern restor ing region does not deviate much from the prescribed profile (see Figure 2-7). The net effect of this parameterized buoyancy forcing in the southern restoring region is to transform deep waters into bottom waters (diabatic downwelling) to balance the transformation of bottom waters into deep waters (diabatic upwelling) driven by mixing along the mid-ocean ridge in the basin to the north. In contrast to $\mathrm{CF} 18$, we allow reference buoyancy profiles $B(z)$ corresponding to verticallyvarying stratification $\partial_{z} B=N^{2}(z)$, complicating the interpretation of the solution in terms of one-dimensional boundary layer dynamics which require a constant interior stratification $N_{0}^{2}$. 

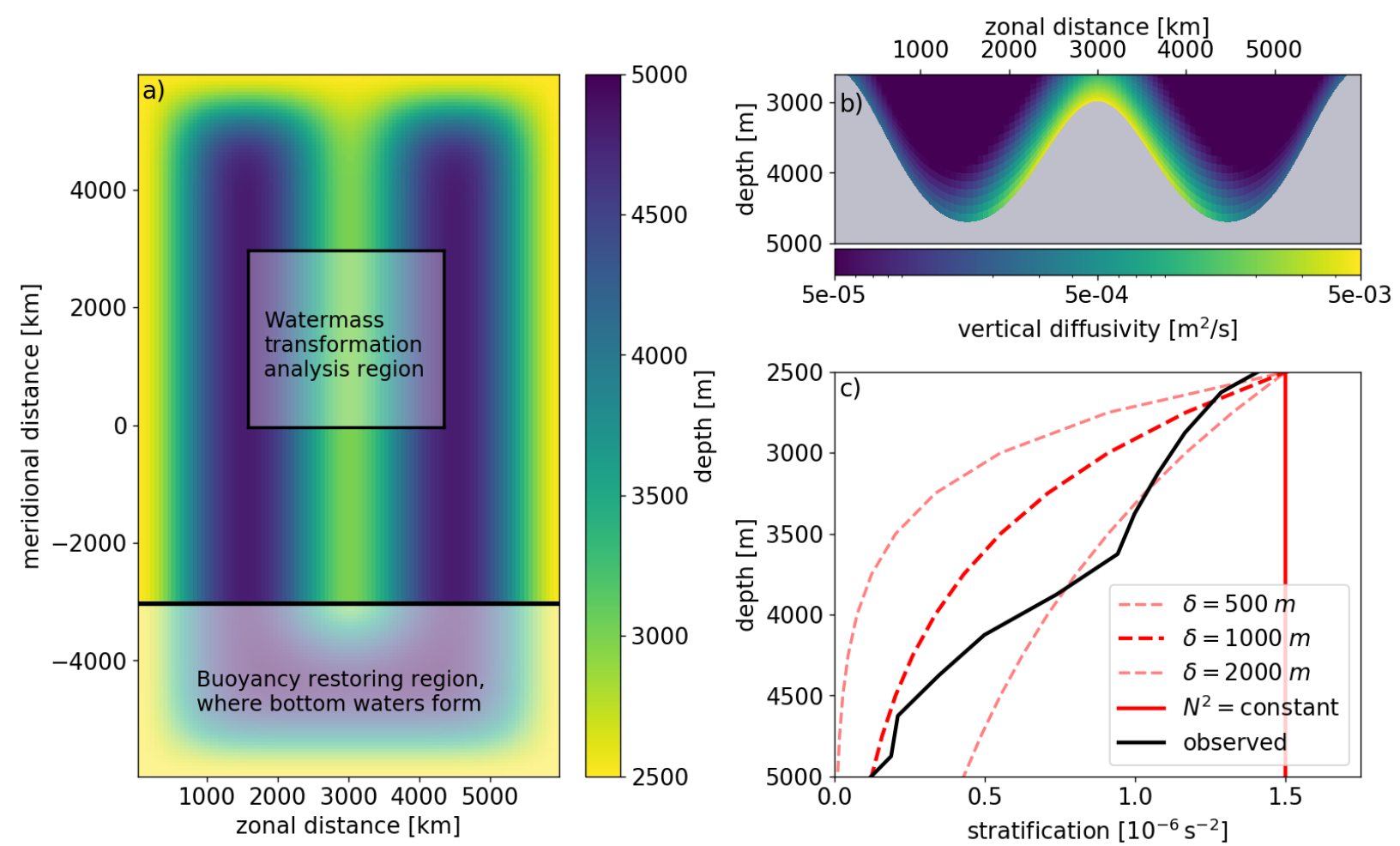

$-4000$

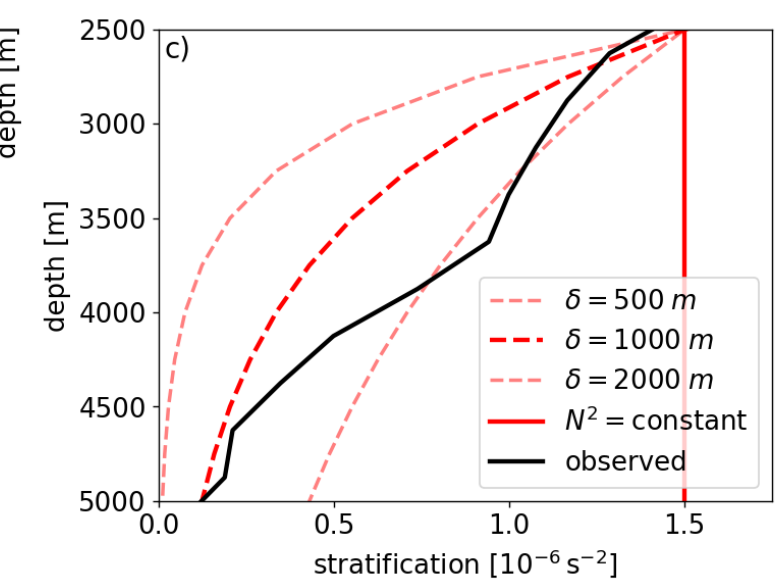

Figure 2-2: Configuration of the Planetary Geostrophic Circulation Model (PGCM). (a) Seafloor depth in the PGCM. We highlight the southern restoring region where we apply a buoyancy restor ing which acts to transform deep waters into bottom waters (see Section 2.3.3) and a northernhemisphere region in which we diagnose watermass transformations along the mid-ocean ridge (see Section 2.4.3). (b) Zonal section of the imposed turbulent diffusivity $\kappa$, which is bottom-enhanced over the mid-ocean ridge. (c) The red lines show the four stratification profiles $B_{z}$ used in the PGCM as both the initial condition and as the reference profile for buoyancy restoring in the southern restoring region. The black line shows the observed stratification profile from the World Ocean Circulation Experiment (Gouretski and Koltermann, 2004) in the South Pacific, averaged horizontally from $55^{\circ} \mathrm{S}$ to $45^{\circ} \mathrm{S}$ and $175^{\circ} \mathrm{E}$ to $115^{\circ} \mathrm{W}$.

\subsubsection{Dimensional parameters and scaling}

While the PGCM is discussed in dimensional terms, the PGCM is formulated and implemented non-dimensionally. The following dimensional scales,

$$
\begin{aligned}
L & =6000 \mathrm{~km} \\
H & =2500 \mathrm{~m}, \\
\beta & =2 \times 10^{-11} \mathrm{~m}^{-1} \mathrm{~s}^{-1}, \\
N^{2} & =1.5 \times 10^{-6} \mathrm{~s}^{-2}, \\
\kappa_{b o t} & =5 \times 10^{-3} \mathrm{~m}^{2} \mathrm{~s}^{-1}, \\
r & =1.2 \times 10^{-5} \mathrm{~s}^{-1},
\end{aligned}
$$

(basin width),

(abyssal ocean vertical extent), (meridional gradient of Coriolis parameter), (reference stratification at $z=-2500 \mathrm{~m}$ ), (diffusivity at the mid-ocean ridge seafloor),

(frictional parameter), 
are used to non-dimensionalize the system, with the coordinate transformation

$$
x=L \hat{x}, \quad y=L \hat{y}, \quad z=H \hat{z}
$$

and the substitutions

$$
\begin{aligned}
& t=\frac{\beta L^{3}}{N^{2} H^{2}} \hat{t}, \quad b=N^{2} H \hat{b}, \quad \quad p=N^{2} H^{2} \hat{p}, \\
& u^{x}=\frac{N^{2} H^{2}}{\beta L^{2}} \hat{u}^{\hat{x}}, \quad u^{y}=\frac{N^{2} H^{2}}{\beta L^{2}} \hat{u}^{\hat{y}}, \quad u^{z}=\frac{N^{2} H^{3}}{\beta L^{3}} \hat{u}^{\hat{z}} .
\end{aligned}
$$

For reference, the non-dimensional time $\hat{t}=1$ corresponds to $t=\tau \simeq 10$ years, where $\tau \stackrel{\text { def }}{\equiv}$ $\beta L^{3} / N^{2} H^{2}$. While the basin scale circulation takes a long time $\tau_{\text {mix }}=H^{2} / \bar{\kappa} \simeq 1000$ years $\gg \tau$ to spin up, the abyssal mixing layers are spun up on a fast timescale $\tau_{\mathrm{BL}}=q^{-2} / \kappa_{\text {bot }} \simeq 1$ year $\ll \tau$, where

$$
q^{-1}=\sqrt{\frac{\kappa_{\text {bot }}\left(f^{2}+r^{2}\right)}{r N^{2} \tan ^{2} \theta}} \simeq 400 \mathrm{~m}
$$

is the thickness of the mixing layer predicted by $1 \mathrm{D}$ theory (CF18), $\kappa_{\text {bot }}=5 \times 10^{-3} \mathrm{~m}^{2} / \mathrm{s}$ is the diffusivity at the seafloor, and $f=\beta L / 2$ is a representative value of the Coriolis parameter.

The non-dimensionalized equations (see CF18) depend only on the non-dimensional parameters

$$
\hat{\alpha}=\frac{H}{L}, \quad \hat{\kappa}=\frac{\kappa \beta L^{3}}{N^{2} H^{4}}, \quad \hat{r}=\frac{r}{\beta L}
$$

where $\hat{\alpha}$ is the aspect ratio of the basin; $\hat{\kappa}=\tau / \tau_{\text {mix }}$ is the ratio of the cross-basin propagation timescale of long Rossby waves (with $f=\beta L$ )

$$
\tau \equiv L / c_{g}=L / \frac{\beta L^{-2}}{(N H / f)^{2}}=\frac{\beta L^{3}}{N^{2} H^{2}}
$$

to the diffusive spin-up timescale $\tau_{\text {mix }} \stackrel{\text { def }}{\equiv} H^{2} / \bar{\kappa} . \hat{r}$ is the ratio of the Stommel (1948) western bound ary layer width $r / \beta$ to the basin width $L$. Since the prescribed $\kappa$ is spatially-dependent, the nondimensional diffusivity $\hat{\kappa}$ inherits its spatial dependence in the numerical implementation. Scaling $\kappa$ by using the volume-weighted average value $\bar{\kappa}$ in $\tau_{\text {mix }}$ gives $\hat{\kappa}=\tau / \tau_{\text {mix }} \simeq 0.01$. Because the imposed turbulent diffusivity is isotropic, the small aspect ratio $\hat{\alpha} \sim 5 \times 10^{-4}$ results in a non-dimensionalized horizontal diffusivity many orders of magnitude smaller than the non-dimensionalized vertical dif fusivity, which is difficult to implement numerically. Instead, we artificially increase the horizontal 
diffusivity for numerical stability by increasing the aspect ratio parameter to $\hat{\alpha}=0.2$. This param eter only enters in the horizontal diffusion term $\hat{\alpha}^{2}\left[\partial_{\hat{x}}\left(\hat{\kappa} \partial_{\hat{x}} \hat{b}\right)+\partial_{\hat{y}}\left(\hat{\kappa} \partial_{\hat{y}} \hat{b}\right)\right](\mathrm{CF} 18)$ and remains small enough that it does not qualitatively affect the results presented here, as evidenced by the negligible role of horizontal buoyancy fluxes in the watermass transformations (Figure 2-5].

\subsubsection{Numerical implementation}

The model is formulated in terrain-following coordinates to accurately resolve the thin mixingdriving flows along the sloped bottom boundary. The numerical implementation is described in CF18. The Julia (Bezanson et al., 2017) implementation is available at https://github.com/ joernc/pgcm. The input files, output files, and post-processing notebooks necessary to replicate the study are available at https://github.com/hdrake/AbyssalFlow (Drake, 2020).

\subsection{Abyssal Circulation Controlled By Mixing Layer Dynamics}

We begin by describing the general structure of the abyssal circulation at equilibrium in the PGCM, i.e. at $\hat{t}=50$ or $t \simeq 500$ years $\simeq \tau_{\text {mix }}$, when buoyancy tendencies have become sufficiently small (Figure 2-7a). The stratification in the PGCM solution presented in this section is restored to an exponential profile with a decay scale of $\delta=1000 \mathrm{~m}$ in the southern region (solid red dashed line in Figure 2-2, which exhibits vertical variations of similar magnitude to those observed in the Southern Ocean (black solid line). This is arguably our most "realistic" simulation of the abyssal ocean and hereafter we refer to it as PGCM-REAL.

\subsubsection{Abyssal Mixing Layers and Deep Western Boundary Currents}

Figure 2-3 (a-c) shows the three Cartesian components of the abyssal flow field along a zonal section $3000 \mathrm{~km}$ north of the equator. In the abyssal mixing layers spanning both flanks of the mid-ocean ridge, buoyancy surfaces plunge to intersect the seafloor at a right angle (visually distorted by the aspect ratio) to satisfy the no-flux boundary condition. As expected from 1D theory (CF18), the boundary flows are thicker and stronger over the mid-ocean ridge where mixing is strong than over the continental slopes where mixing is weak. In the bottom boundary layer (BBL), plunging buoyancy surfaces drive frictionally-balanced upwelling (Figure 2-3c) and frictional-geostrophic flow opposite the direction of Kelvin wave propagation (Figure 2-3b), i.e. anti-cyclonic in the northern hemisphere. In the stratified mixing layer (SML) just above the BBL, buoyancy surfaces are at 
leading order flat and the bottom-enhanced mixing drives downwelling (Figure 2-3e), as expected from the vertical advection-diffusion balance (eq. 2.3) reviewed in Section 2.2.
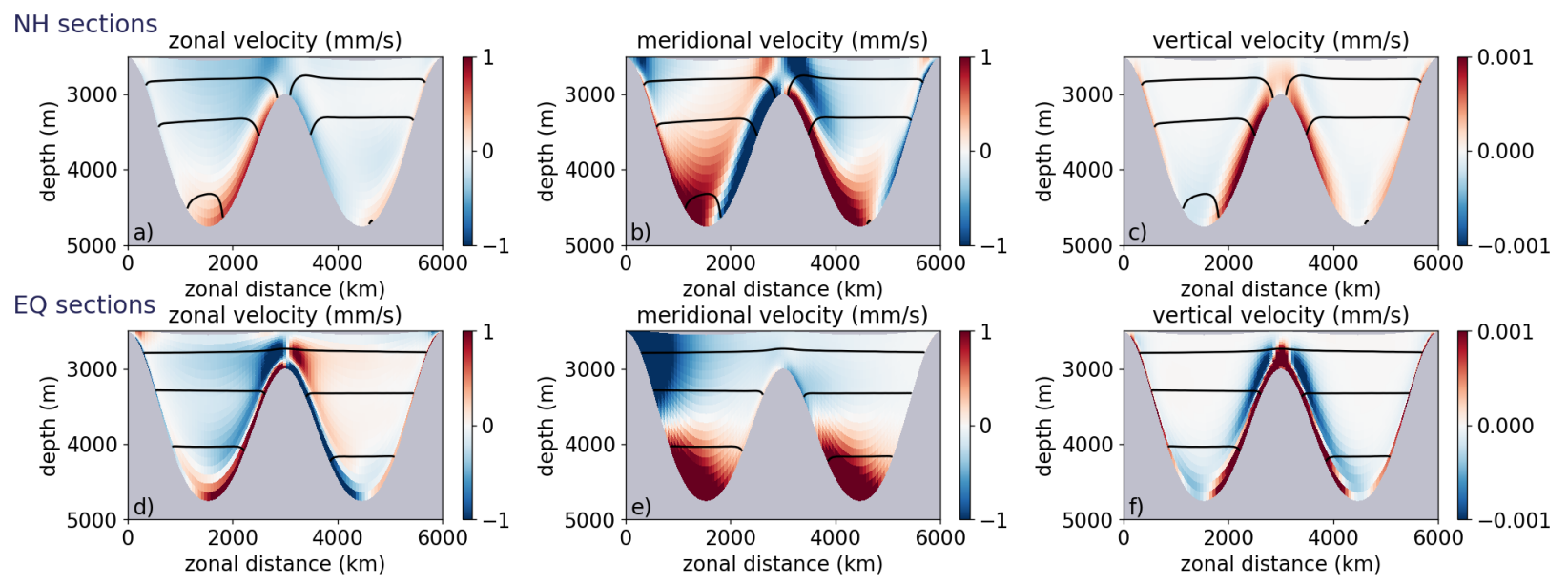

Figure 2-3: Cartesian components of the velocity vector in the PGCM-REAL simulation along: (a-c) a mid-latitude section in the northern hemisphere, $y=3000 \mathrm{~km}$, and (d-f) a section at the equator, $y=0 \mathrm{~km}$. Black lines show three equally spaced buoyancy surfaces.

Net diapycnal upwelling in the Northern Hemisphere can be inferred from the meridional flow field at the equator: dense bottom waters flow into the northern hemisphere and relatively lighter deep waters flow out (Figure 2-3 ). Since the Coriolis force vanishes at the equator, the buoyant force associated with the bending of buoyancy surfaces to satisfy the bottom-boundary condition can only be balanced by a cross-slope frictional flow (Figure 2-3 $\mathrm{d}, \mathrm{f}$ ) and any along-slope flows associated with the abyssal mixing layers vanishes (compare Figure 2-3 to Figure 2-3b). The only meridional flow are Stommel (1948)-like deep western boundary currents (DWBC) along the continental slope on the western side of the domain and the eastern flank of the ridge (Figure 2-3e). In this particular configuration, a southward-flowing DWBC develops on the eastern flank of the ridge near its crest and is much weaker than the DWBC on the western continental slope. The southward DWBC on the ridge is relatively intensified in simulations with a taller ridge.

\subsubsection{Depth-integrated and Overturning Circulations}

The global abyssal circulation is more intuitively visualized by considering the three Cartesian streamfunctions that describe the flow, which we compute by integrating the $u^{x}, u^{y}$, and $u^{z}$ velocities 
in the other two dimensions ${ }^{5}$ (Figure 2-4). Figure 2-4 shows the familiar streamfunction for the meridional overturning circulation (MOC) in the $y-z$ plane, which should be thought of as corresponding to the lower-cell of the global MOC. This circulation has a strength of about $1.6 \mathrm{~Sv}$ at the equator, with water 1) downwelling diabatically in the southern restoring region, 2) flowing northwards to fill the abyssal depths, 3) gradually upwelling along the length of the basin, and 4) returning to the southern restoring region to close the circulation. We note in particular that the MOC extends all the way from the ocean seafloor to the top of the mid-ocean ridge, in contrast to the MOC in the CF18 framework, in which significant overturning is confined to the base of topographic slopes (see Section 2.6.3 for a discussion on the role of the ridge height in setting the vertical extent of the MOC).
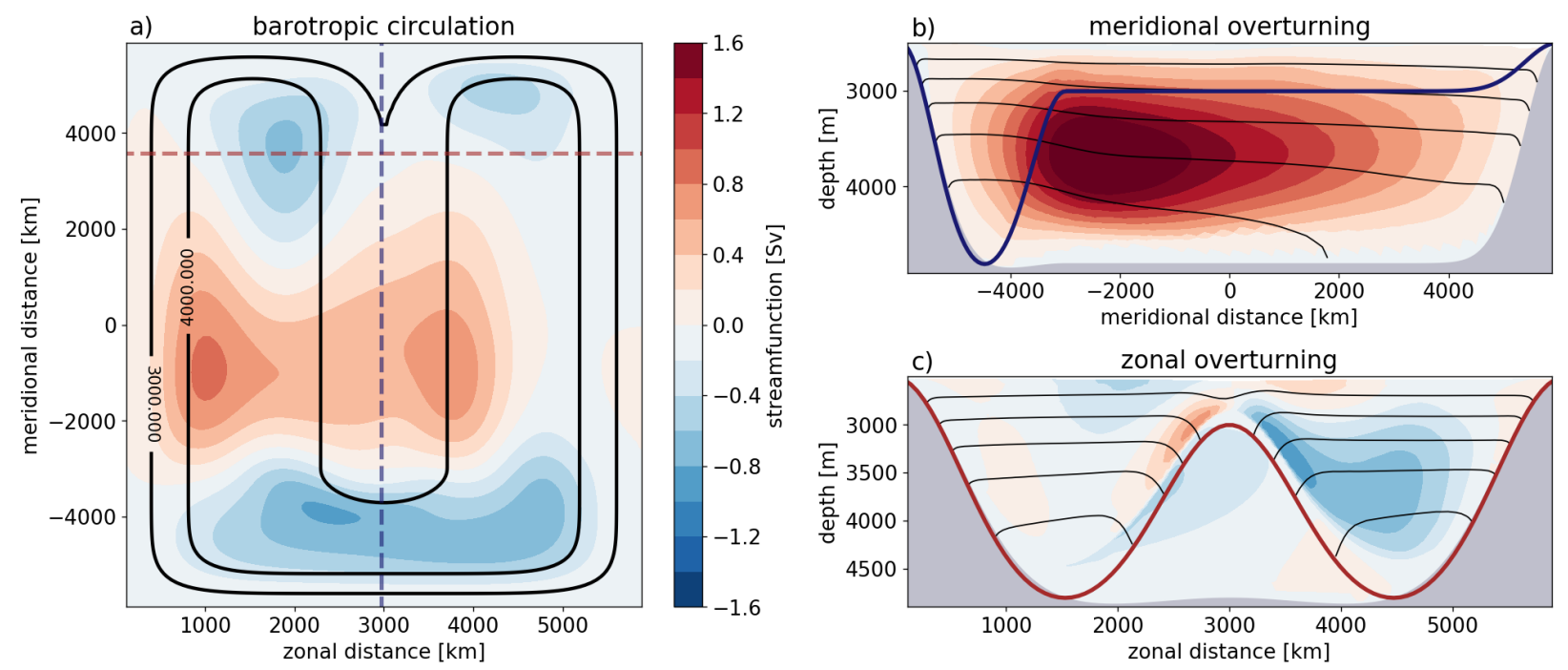

Figure 2-4: (a) Barotropic, (b) meridional, and (c) zonal overturning circulations in the PGCM REAL simulation (see definitions in 2.4.2). In all cases, positive values (red) correspond to counter clockwise circulations. The blue and red solid lines in (b) and (c), respectively, show the height of the mid-ocean ridge along the dashed lines of the same colors in (a). The thick black lines in (a) are the $3000 \mathrm{~m}$ and $4000 \mathrm{~m}$ isobaths, which highlight both the continental slopes and the mid-ocean ridge. The thin black lines in (b) and (c) are equally-spaced buoyancy surfaces, sampled at $x=L / 4$ and $y=L / 2$, respectively.

The up- and down-welling in the abyssal mixing layers is evident in the zonal overturning streamfunction in the $x-z$ plane, which shows upwelling in a thin BBL and broader downwelling ${ }^{5}$ Integrating the continuity equation in $\frac{\partial u^{x}}{\partial x}+\frac{\partial u^{y}}{\partial y}+\frac{\partial u^{z}}{\partial z}=0$ along any of the three directions $x, y$, or $z$ and imposing the no-normal flow boundary condition yields an equation of the form $\int\left(\frac{\partial u^{x_{1}}}{\partial x_{1}}+\frac{\partial u^{x_{2}}}{\partial x_{2}}+\frac{\partial u^{x_{3}}}{\partial x_{3}}\right) \mathrm{d} x_{3}=$ $\frac{\partial U^{x_{1}}}{\partial x_{1}}+\frac{\partial U^{x_{2}}}{\partial x_{2}}=0$, where $x_{1}, x_{2}, x_{3}$ are permutations of $x, y, z, U^{x_{1}}=\int u^{x_{1}} \mathrm{~d} x_{3}$ and $U^{x_{2}}=\int u^{x_{2}} \mathrm{~d} x_{3}$. The resulting non-divergent flow field can then be expressed as a streamfunction $\psi_{3}$ defined by $\mathbf{U}=U^{x_{1}} \mathbf{x}_{\mathbf{1}}+U^{x_{2}} \mathbf{x}_{\mathbf{2}}=\left(-\nabla \times \psi_{3} \mathbf{x}_{\mathbf{3}}\right)$. 
in the SML above (Figure 2-4c). The upwelling in bottom boundary layers is confined to the two flanks of the mid-ocean ridge, where mixing is vigorous and bottom-enhanced, and is negligible over the weakly-mixed continental slopes. In this case, the upwelling and downwelling transports are equal and opposite in strength, i.e. the circulation closes, because the downwelling flow includes both the residual diabatic upwelling along the ridge as well as the net diabatic downwelling by the restoring condition in the southern region, which is concentrated on the eastern continental slope. Nonetheless, the zonal overturning streamfunction provides a qualitative sense of the zonal overturning circulations driven by mixing layer dynamics along the mid-ocean ridge.

The depth-integrated circulation in our simulations stands in contrast to that of Stommel and Arons (1959a)'s barotropic model and is the expression of a combination of various baroclinic deep western boundary currents and mixing layer flows (Figure 2-4 $)$. Within $2000 \mathrm{~km}$ of the equator, the northward and southward components of the deep western boundary currents alternatively dominate (compare with the meridional velocity at the equator in Figure 2-3). North of $y=2000 \mathrm{~km}$, the depth-integrated circulation is dominated by the along-slope flow in the bottom boundary layer, which is opposite the direction of Kelvin wave propagation. The depth-integrated circulation is strongly influenced by mixing layer dynamics, both near the boundaries and in the interior, and is structurally distinct from that predicted by the linear response to vortex stretching alone (Stommel and Arons, 1959b; Pedlosky, 1992; Cember, 1998). Sverdrup balance only holds far from the boundaries and accounts for little of the net transport compared to the abyssal mixing layer and western boundary current flows, where friction is important.

\subsubsection{Partially-Compensating Watermass Transformations}

Watermass transformation represents the net flow across a buoyancy surface driven by diabatic forcing. Watermass transformation in the PGCM is driven by: 1) bottom-enhanced turbulent mixing (positive in the net) and 2) restoring to a reference buoyancy profile in the southern restoring region (negative in the net). In the northern hemisphere, the restoring rate vanishes by construction and watermass transformation is dominated by the mixing-driven component. Its calculation, following Walin (1982) and Ferrari et al. (2016), is given by

$$
T(b)=\frac{\partial}{\partial b} \int_{V_{b^{\prime}<b}} \nabla \cdot\left(\kappa \nabla b^{\prime}\right) \mathrm{d} V
$$


where $V_{b^{\prime}<b}$ is the volume of water less buoyant than $b$. Watermass transformation is conveniently expressed in units of volumetric transport $\left(\mathrm{m}^{3} / \mathrm{s}\right)$ and can be decomposed into various contributions. When applied to regions of bottom-enhanced mixing in the abyss, it is informative to decompose the net watermass transformation into the typically negative contribution (balanced by diapycnal downwelling) in the SML and the typically positive contribution (balanced by diapycnal upwelling) in the BBL (e.g. Ferrari et al., 2016; McDougall and Ferrari, 2017). For the purposes of watermass transformation calculations in this paper, we define the BBL as the layer with a convergent buoyancy flux, $\nabla \cdot(\kappa \nabla b)>0$, which extends upwards from the seafloor to the level at which the buoyancy flux attains its maximum magnitude; the remainder of the ocean is considered the SML and is dominated by a buoyancy flux divergence, $\nabla \cdot(\kappa \nabla b)<0$. For convenience, all watermass transformations in this paper are computed in buoyancy space and remapped into depth space according to the average depth of buoyancy surfaces,

$$
\bar{z}(b)=\frac{1}{A(b)} \int z(b) d A,
$$

which facilitates comparison across simulations with dramatically different stratifications and against the fixed depths of topographic features in the ocean.
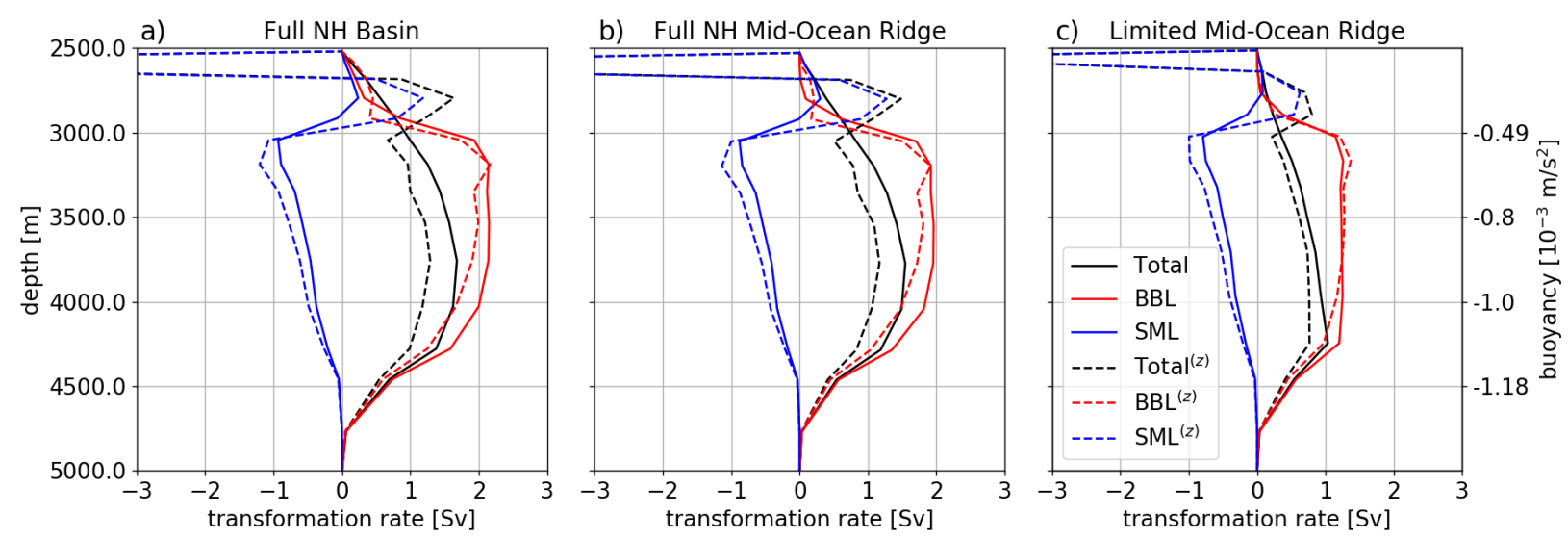

Figure 2-5: Watermass transformations from the PGCM-REAL simulation in (a) the full northern hemisphere basin $(y>0)$, (b) along the northern-hemisphere mid-ocean ridge $(y>0, L / 2<x<$ $3 L / 2$ ), and (c) in a limited watermass analysis region along the northern-hemisphere mid-ocean ridge $(0<y<L / 2, L / 2<x<3 L / 2)$, as highlighted in Figure 2-2. The black line shows the net watermass transformation, defined by equation (2.18). The red and blue lines show the contributions from the bottom boundary layer (BBL) and the stratified mixing layer above (SML). The dashed lines show the contributions from only the vertical component of the buoyancy flux. All watermass transformations in the paper are computed in buoyancy space and remapped into depth space (according to eq. 2.19.

The net northern hemisphere watermass transformation of $T_{\text {net }}=1.6 \mathrm{~Sv}$ at $3750 \mathrm{~m}$ (Figure 
2-5a), where it reaches its maximum, is consistent with the depth and magnitude of the maximum of the MOC streamfunction at the equator (Figure 2-4 $\mathrm{b}$ ). The net watermass transformation $T_{\text {net }}=$ $T_{\mathrm{BBL}}+T_{\mathrm{SML}}=1.6 \mathrm{~Sv}$ (black line) is the residual of a positive contribution of $T_{\mathrm{BBL}}=2.1 \mathrm{~Sv}$ from the $\mathrm{BBL}$ (red line) and a negative contribution of $T_{\mathrm{SML}}=-0.5 \mathrm{~Sv}$ from the SML (blue line), both of which are dominated by the vertical component of the buoyancy flux divergence (dashed lines), i.e.

$$
T(b)=\partial_{b} \int_{V_{b^{\prime}<b}} \nabla \cdot\left(\kappa \nabla b^{\prime}\right) \mathrm{d} V \simeq \partial_{b} \int_{V_{b^{\prime}<b}} \partial_{z}\left(\kappa \partial_{z} b^{\prime}\right) \mathrm{d} V .
$$

Virtually all of this transformation occurs on the flanks of the mid-ocean ridge (compare Figure $2-5 a, b)$.

For the convenience of being able to ignore meridional variations in the basin geometry (and their effects on watermass transformations via the "perimeter" effect, as described by Holmes et al. 2018), we limit the remaining discussion to a domain from $L / 2<x<3 L / 2$ and $0<y<L / 2$ along the north-hemisphere mid-ocean ridge, which is responsible for roughly 1 Sv of the full basin's transformation (Figure 2-5; limited domain outlined in Figure 2-2a).

The net watermass transformation $T_{\text {net }}=1 \mathrm{~Sv}$ at equilibrium is much larger than the $L \Psi_{\mathrm{bg}} \leq$ $0.1 \mathrm{~Sv}$ predicted by the integral constraint (eq. 2.5) from $1 \mathrm{D}$ boundary layer theory. To clarify the discrepancy between the watermass transformations that emerge from the 3D PGCM and the watermass transformations predicted by 1D dynamics, we emulate the 3D PGCM simulation by solving the 1D boundary layer equations locally and interpolating them onto the 3D PGCM grid.

\subsection{Emulating the 3D PGCM with local 1D boundary layer models}

\subsubsection{Boundary layer theory}

Following CF18, we transform the planetary-geostrophic equations 2.6 - 2.8 from the Cartesian coordinates $(x, y, z)$ to a coordinate system $\left(x^{\prime}, y^{\prime}, z^{\prime}\right)$ aligned with an infinitely extending sea floor at $z=x \tan \theta$, with slope angle $\theta$, and ignoring the southern region restoring condition on buoyancy. The transformation is given by $x^{\prime}=x \cos \theta+z \sin \theta, y^{\prime}=y, z^{\prime}=-x \sin \theta+z \cos \theta$. Buoyancy $b=B(z)+b^{\prime}$ is decomposed into a background $B(z)$ with constant stratification $\partial_{z} B=N_{0}^{2}$ and an 
anomaly $b^{\prime}\left(z^{\prime}\right)$. The planetary-geostrophic boundary layer equations are thus given by:

$$
\begin{aligned}
-f \cos \theta u^{y^{\prime}} & =b^{\prime} \sin \theta-r \cos \theta^{2} u^{x^{\prime}} \\
f \cos \theta u^{x^{\prime}} & =-r u^{y^{\prime}} \\
\partial_{t} b+u^{x^{\prime}} N_{0}^{2} \sin \theta & =\partial_{z^{\prime}}\left[\kappa\left(N^{2} \cos \theta+\partial_{z^{\prime}} b^{\prime}\right)\right],
\end{aligned}
$$

with a no-flux boundary condition $\partial_{z^{\prime}} b^{\prime}+N_{0}^{2} \cos \theta=0$ at the seafloor $z^{\prime}=0$ and decay conditions

$\partial_{z^{\prime}} u^{x^{\prime}}, \partial_{z^{\prime}} u^{x^{\prime}}, \partial_{z^{\prime}} b^{\prime} \rightarrow 0$ as $z^{\prime} \rightarrow 0$. At steady state, these equations yield exact analytical solutions for constant $\kappa(\mathrm{CF} 18)$ and approximate analytical solutions for elementary $\kappa(z)$ profiles $($ Dell, 2013 , Callies, 2018).

\subsubsection{Emulator setup}

We emulate the PGCM solution by using finite differences to solve the time-dependent boundarylayer equations 2.21) - 2.23) with the local Coriolis parameter $f(y)$ and slope angle $\theta(x, y)$ at each $(x, y)=(\xi, \eta)$ of the PGCM grid, which is a sensible approach given that the parameters $f(y)$ and $\theta(x, y)$ vary on scales larger than the those of the boundary-layer solutions (Dell and Pratt, 2015). Since these local boundary layer solutions are given in terms of the local slope-normal direction $z^{\prime}$ rather than the true vertical direction $z$, we project the solution onto the true vertical direction $z$ with the substitution $z^{\prime} \rightarrow z / \cos \theta$ and linearly interpolate from the projected $z$-levels of the boundary layer solution to the PGCM's local $\sigma$-levels. This process provides an emulator of the PGCM which is purely the result of local $1 \mathrm{D}$ dynamics but is re-gridded to the same grid as the 3D PGCM and can thus be directly compared. While the emulator does not conserve mass or other properties, it does yield insight into the structure and dynamics of the 3D solution.

\subsubsection{Emulator evaluation}

We evaluate the emulator against the spin-up of a PGCM simulation with a constant stratification initial condition $N_{0}^{2}$, hereafter PGCM-CONST. The 1D emulator accurately reproduces the initial spin-up of buoyancy and velocity fields of the PGCM-CONST simulation along most of the midocean ridge flanks, but fails at the top and bottom of the ridge where the topographic curvature is large and the cross-slope convergences omitted by 1D dynamics become important (Figure 2-6a,b).

As the solution nears equilibrium, however, the interior basin stratification drifts away from its constant initial value (compare gray and black contours in Figure 2-6 d) and the boundary layer 

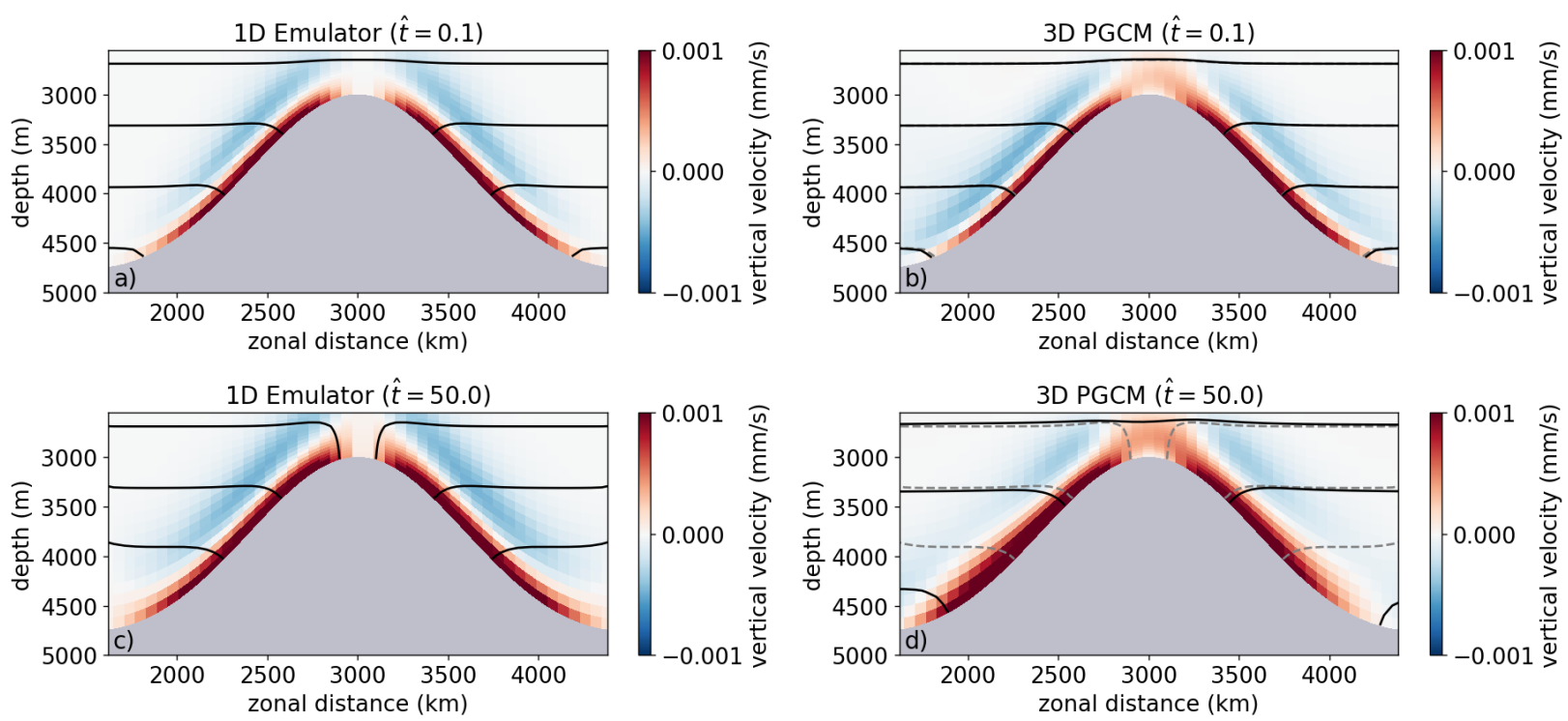

Figure 2-6: Vertical velocity (colors) and buoyancy surfaces (black lines) in abyssal mixing layers along a zonal section across the mid-ocean ridge at $y=L / 2$ in $(\mathrm{a}, \mathrm{c})$ the $1 \mathrm{D}$ PGCM emulator and (b,d) the full 3D PGCM, where both are initialized from identical constant stratification buoyancy fields. The top row shows the solutions at an initial time $\hat{t}=0.1$, at which point the abyssal mixing layers have spun up but the basin-scale circulation has not (see Section 2.3.4). The bottom row shows the solution at $\hat{t}=50$, at which point the full solution has roughly come to equilibrium with the buoyancy restoring in the Southern Ocean. The predicted buoyancy surfaces in (a,c) are reproduced as dashed grey lines in $(\mathrm{b}, \mathrm{d})$ to show how the $1 \mathrm{D}$ emulator predicts the buoyancy field well for short times but that the interior stratification in the PGCM drifts far from the 1D emulator's prediction as it approaches equilibrium.

flows diverge from the 1D emulator's prediction (Figure 2-6e,d). This is expected, as the basin stratification of PGCM-CONST is allowed to evolve in response to the 3D circulation while the background interior stratification $N_{0}^{2}$ is a constant parameter in the emulator 6 . Relative to the emulator, the equilibrium PGCM-CONST solution exhibits reduced downwelling in the SML and enhanced upwelling in the BBL, both of which contribute to enhancing the net diapycnal upwelling. In Section 2.6, we use the 1D emulator to identify properties of the watermass transformations in the PGCM that can be explained by one-dimensional dynamics alone.

\footnotetext{
${ }^{6}$ An attempt was made to correct for this by imposing the equilibrium stratification of the 3D model as the farfield stratification for the 1D emulator, but the results were underwhelming (not shown) and suggest instead that the coupling between boundary layers and the interior is at least a first-order if not leading order effect.
} 


\subsection{The Effect of Variable Interior Stratification on the Abyssal Circulation}

\subsubsection{What sets the abyssal stratification?}

In our PGCM simulations, the drift of interior buoyancy surfaces over time (Figure 2-6d) suggests that the interior stratification at equilibrium may differ substantially from the stratification of the southern region buoyancy profile. Figure 2-7a shows the temporal evolution of the horizontallyaveraged vertical stratification profile, averaged over the northern hemisphere basin in the PGCM, where darker greys represent later times. In PGCM-CONST, the abyssal stratification develops sub stantial vertical structure in the basin over time, despite being rapidly restored back to a constant stratification in the southern restoring region (solid lines, Figure 2-7 $\mathrm{a}$ ). Net watermass transfor mation is initially unbalanced by Eulerian diapycnal flow (compare Figure 2-8b,f) and thus drives changes in the volume of buoyancy layers, which can be interpreted as a component of the diapy cnal transport due to the velocity of buoyancy surfaces (Marshall et al., 1999). Excess watermass transformations near the base of the slope destroy the densest layers and expand the deep layers, which translate into a reduction of the stratification that originates at the bottom of the ridge and propagates upwards over time. The details of the vertical structure of the equilibrium basin stratification depend on ridge height (not shown), but in all cases the basin stratification increases from zero at the maximum depth (imposed by the no-flux condition at the flat bottom) up to near the restoring reference value of $N_{0}^{2}$ at the top boundary.

The zonal-mean basin stratification develops a significant meridional structure, wherein the zonal-mean stratification along the ridge weakens with distance from the Southern restoring region (Figure 2-7b,c). In contrast to the mixing layer stratification, which is strongest at the equator and weaker polewards (Callies, 2018, CF18), the zonal-mean stratification decreases roughly monotoni cally with increasing latitude.

Simulations using a reference buoyancy profile that corresponds to an exponential stratification

with decay scale of $\delta=1000 \mathrm{~m}$ exhibit much less drift in their stratifications over time (dashed lines, Figure 2-7a,b). Although the equilibrium basin stratifications in all of the different PGCM experiments develop vertical structure, there does not seem to be a single preferred equilibrium stratification that depends only on the mixing: both the geometry of the abyssal topography and the restoring profile in the southern restoring region influence the interior stratification at equilibrium. 

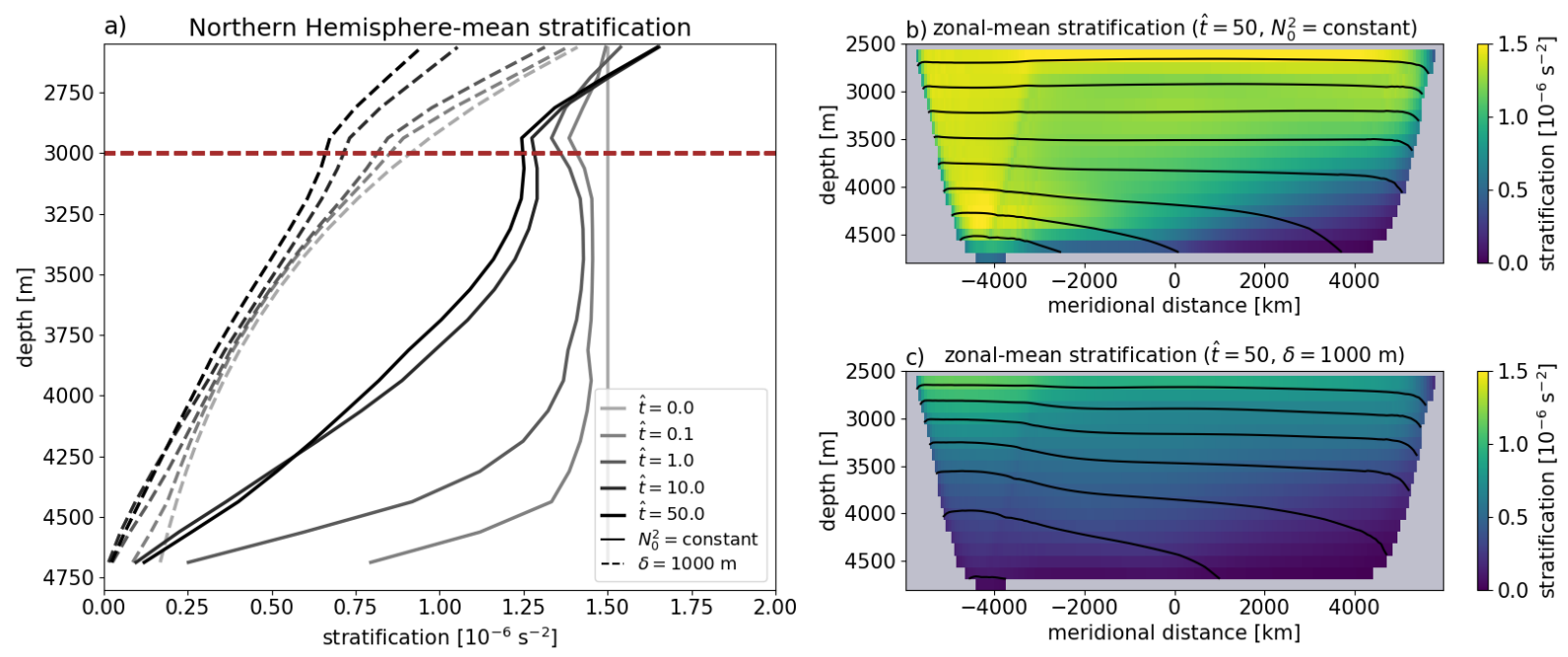

Figure 2-7: (a) Temporal evolution and (b,c) meridional structure of the stratification in PGCM simulations. Grey-scale lines in (a) show the temporal evolution of the horizontal-mean stratification in the Northern Hemisphere for simulations with a constant stratification restoring buoyancy profile (solid lines) and a restoring buoyancy profile corresponding to stratification that decays with depth with a scale height of $\delta=1000 \mathrm{~m}$ (dashed lines). The dashed brown line delineates the height of the ridge crest. Panels (b) and (c) show the zonal-mean stratification at equilibrium $\hat{t}=50$ (colors) and equally-spaced buoyancy surfaces (black lines) for experiments with restoring to constant and exponential stratification, respectively.

\subsubsection{Effect of variable stratification on watermass transformations}

We begin by considering the case of transient spin-up from a reference buoyancy profile with con stant stratification $N_{0}^{2}$, PGCM-CONST. It is useful to consider the evolution of the PGCM during its initial spin-up $\left(\hat{t} \simeq \tau_{\mathrm{BL}} / \tau=0.1\right)$ when only mixing layer dynamics are relevant and the solution is thus well-predicted by the 1D Emulator (Figure 2-6). Figure 2-8a shows the watermass transforma tions in the 1D Emulator at $\hat{t}=0.1$, which almost exactly predicts the watermass transformations in the full 3D PGCM (Figure 2-8p).

Between $-4200 \mathrm{~m}<z<-3000 \mathrm{~m}$, where the slope of the mid-ocean ridge is roughly constant, the near-boundary flow exhibits a vanishingly small net transport (solid black line in Figure 2-8a), which is approximately equal to the integral constraint $T_{\text {net }} \simeq L \Psi_{\text {bg }}=L \kappa_{\text {bg }} \cot \theta \leq 0.1 \mathrm{~Sv}$ predicted by $1 \mathrm{D}$ boundary layer theory (dashed black line in Figure 2-8 a). This vanishingly small net transport is the result of large positive transformation $T_{\mathrm{BBL}}$ (diabatic upwelling, in red) in the $\mathrm{BBL}$ and almost-as-large negative transformation $T_{\mathrm{SML}}$ (diabatic downwelling, in blue) in the SML. Below $z=-4200 \mathrm{~m}$, at the base of the topographic slope, abyssal bottom waters feed the upwelling in the BBL and the maximum net watermass transformation is well predicted by the strictly upwelling 

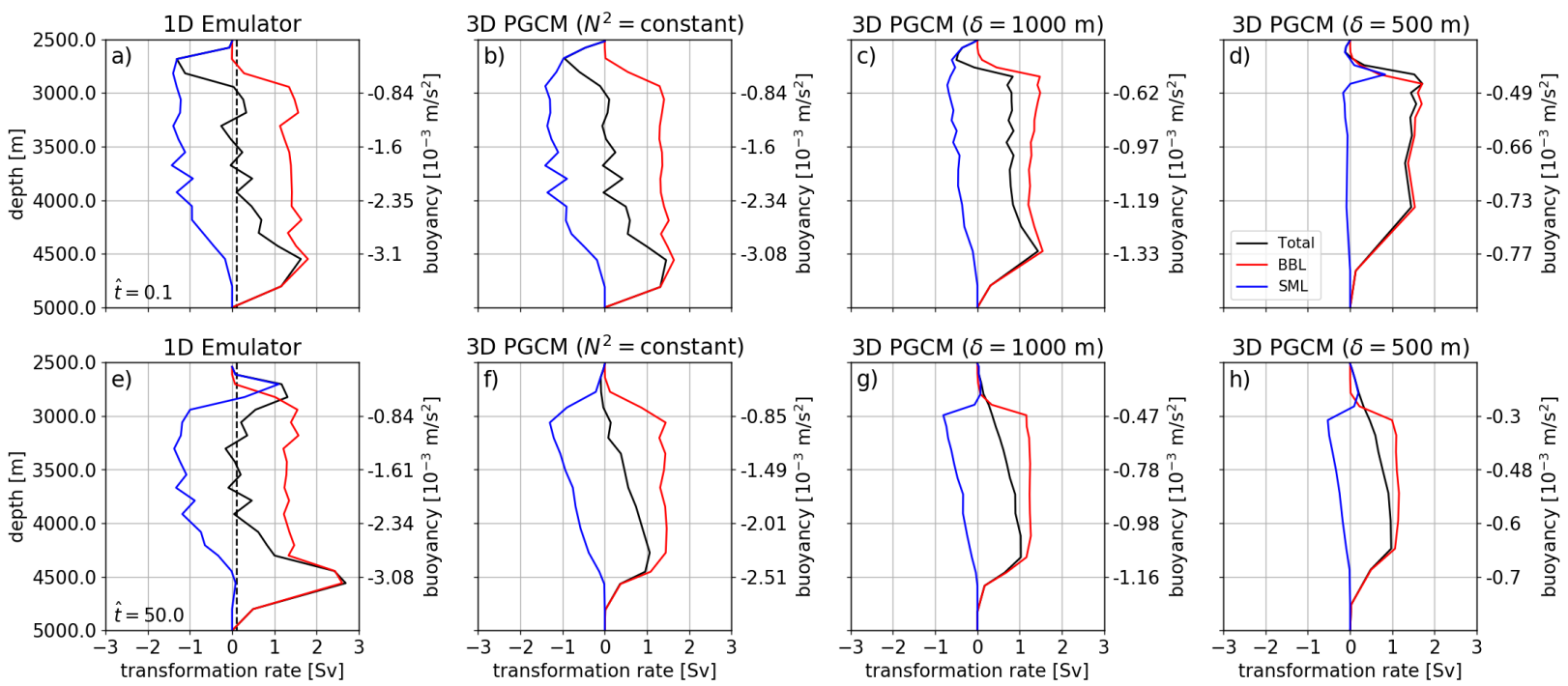

Figure 2-8: Watermass transformations in: (a, e) a 1D emulator of the PGCM and (b-d, f-h) the 3D PGCM simulations with restoring buoyancy profiles corresponding to stratification profiles with various exponential scale heights $\delta$ (we recover $N^{2}=$ constant as $\delta \rightarrow \infty$ ). The initial spin-up at $\hat{t}=0.1$ is shown in (a-d) and the equilibrium state at $\hat{t}=50$ is show in (e-h). Black, red, and blue lines show the net, bottom boundary layer (BBL), and stratified mixing layer (SML) contributions to the watermass transformations, respectively. The black dashed line in (a,e) shows the integral constraint $L \Psi_{\infty}=L \kappa_{\text {bg }} \cot \theta_{\max }$ derived from boundary layer theory, where we take $\theta_{\max }$ as the maximum slope angle of the mid-ocean ridge flank.

transport in the bottom boundary layer from 1D theory (Figure 2-8 a,b), as suggested by CF18.

For the spin-up from a reference stratification that increases exponentially with height (as is almost ubiquitously the case in the abyssal ocean), the integral constraint (eq. 2.5) no longer holds at $\hat{t}=0.1$ and the solution already exhibits a net transformation much larger than $L \Psi_{\mathrm{bg}}$ at all depths from the base of the slope to the ridge crest (Figure 2-8c,d). The increase in the net transformation, which spans the full vertical extent of the ridge, is primarily due to a decrease in the downwelling in the SML which, in the extreme case of an exponential scale height of $\delta=$ $500 \mathrm{~m}$ for the restoring stratification, vanishes completely (Figure 2-8 d). The strongly positive net transformation is primarily due to the buoyancy convergence driven by the rapid increase of the initial stratification with height, i.e. $\kappa \partial_{z z} B>0$ reduces the divergence $\nabla \cdot(\kappa \nabla b)<0$ due to $\partial_{z} \kappa<0$ in the SML (see also Figure 2-14).

As these solutions reach equilibrium, they retain a finite net transformation at all depths from the base of the slope to the ridge crest, slightly reduced by gradually strengthening negative trans formations in the SML (compare Figure $2-8 \mathrm{~s}, \mathrm{~h}$ to Figure 2-8 $\mathrm{k}, \mathrm{d}$ ). At equilibrium, we find the degree of compensation near the ridge crest depends on the vertical scale over which the restoring stratifi 
cation varies (within a range applicable to the ocean): the more rapidly the stratification increases with height, the less upwelling in the BBL is compensated by downwelling in the SML (Figure 2-8f-h and Figure 2-9a). In contrast, upwelling in the BBL is remarkably invariant to vertical variations in the stratification and remains a reasonable prediction for the maximum net transformation (Figure 2-9 a,b), which occurs at the base of the slope where the compensating downwelling contribution from the SML vanishes (Figure 2-9 ). Thus, while the maximum net watermass transformation is accurately predicted by upwelling in the BBL alone, the vertical structure and extent of watermass transformations depend also on downwelling in the SML, which itself is strongly dependent on the vertical stratification, and is not predicted by $1 \mathrm{D}$ theory.
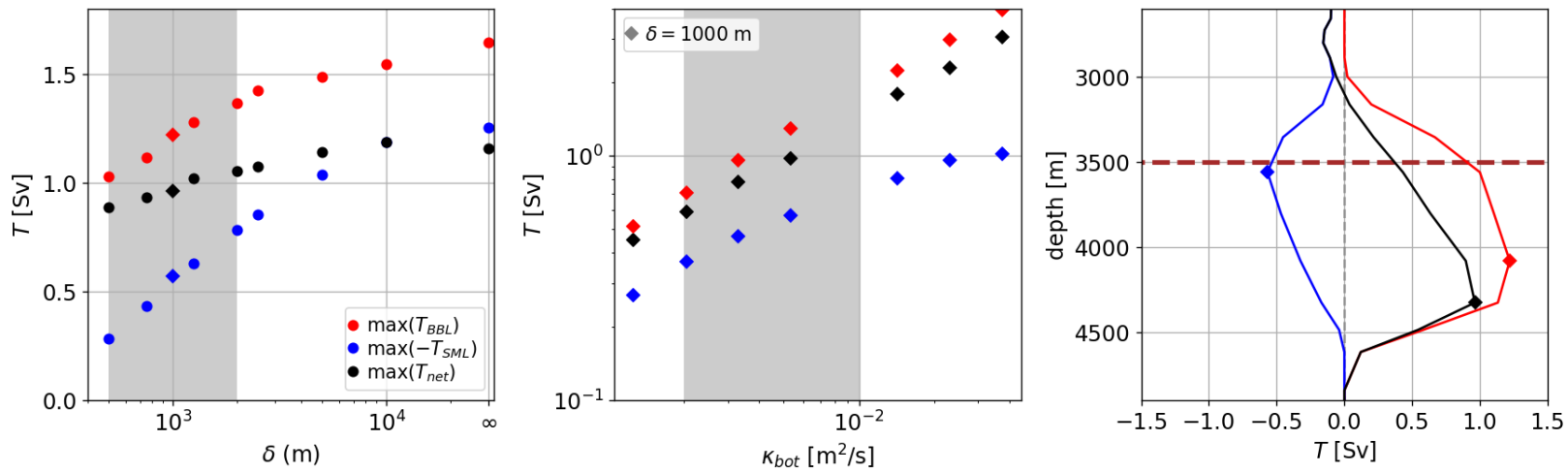

Figure 2-9: Scaling of watermass transformations in mixing layers with (a) the height $\delta$ over which the restoring stratification varies and (b) the bottom diffusivity $\kappa_{\text {bot }}$. Colored symbols represent the absolute value of the maximum transport in the BBL (red), the SML (blue), and the net (black), with diamonds representing the PGCM-REAL simulations. The grey shading in (a) represents realistic vertical scales $\delta$ over which abyssal stratification varies and in (b) represents plausible values of the bottom diffusivity $\kappa_{\text {bot }}$. Panel (c) provides an example of the watermass transformations for a bottom diffusivity $\kappa_{\text {bot }}=5 \times 10^{-3} \mathrm{~m}^{2} \mathrm{~s}^{-1}$, a stratification height scale $\delta=1000 \mathrm{~m}$, and a ridge height of $r_{h}=1500 \mathrm{~m}$, where the corresponding maxima are marked by diamonds and the dashed brown line represents the ridge crest. In all experiments, the maximum net and BBL transformations occur at depths of roughly $4250 \mathrm{~m}$, at the base of the ridge slope, while the maximum SML transformation occurs at the ridge crest.

\subsubsection{Vertical extent of overturning set by ridge height}

We have shown that most of the watermass transformation occurs within abyssal mixing layers along the mid-ocean ridge (Figure 2-5a,b). We further hypothesize that variations in the height of the ridge modulate the vertical extent of abyssal watermass transformations and thus the vertical extent of the abyssal overturning cell. We test this hypothesis by running variations of the PGCM CONST where we vary the ridge height from $500 \mathrm{~m}$ to $2000 \mathrm{~m}$, in increments of $500 \mathrm{~m}$. In the 
initial spin-up, largely compensating positive and negative transformations develop in the BBL and SML, respectively, from the base of the ridge slope up to the ridge crest (Figure 2-10a-d). The net transformation below the ridge crest vanishes according to the integral constraint (eq. 2.5), except near at the base of the ridge where bottom water feeds into the BBL. At equilibrium, however, the stratification drifts away from its constant reference state (e.g. Figure 2-7) and permits a finite net transformation (Figure 2-10 -h), which spans the full vertical extent of the ridge. The result that the vertical extent of the abyssal MOC follows the vertical extent of the mid-ocean ridge is consistent with Lumpkin and Speer (2007)'s global inversion for the MOC, which shows that the vertical extent of the Atlantic and Indo-Pacific lower MOC cells appear to closely follow the vertical extent of their respective major bathymetric features (i.e. mid-ocean ridges).
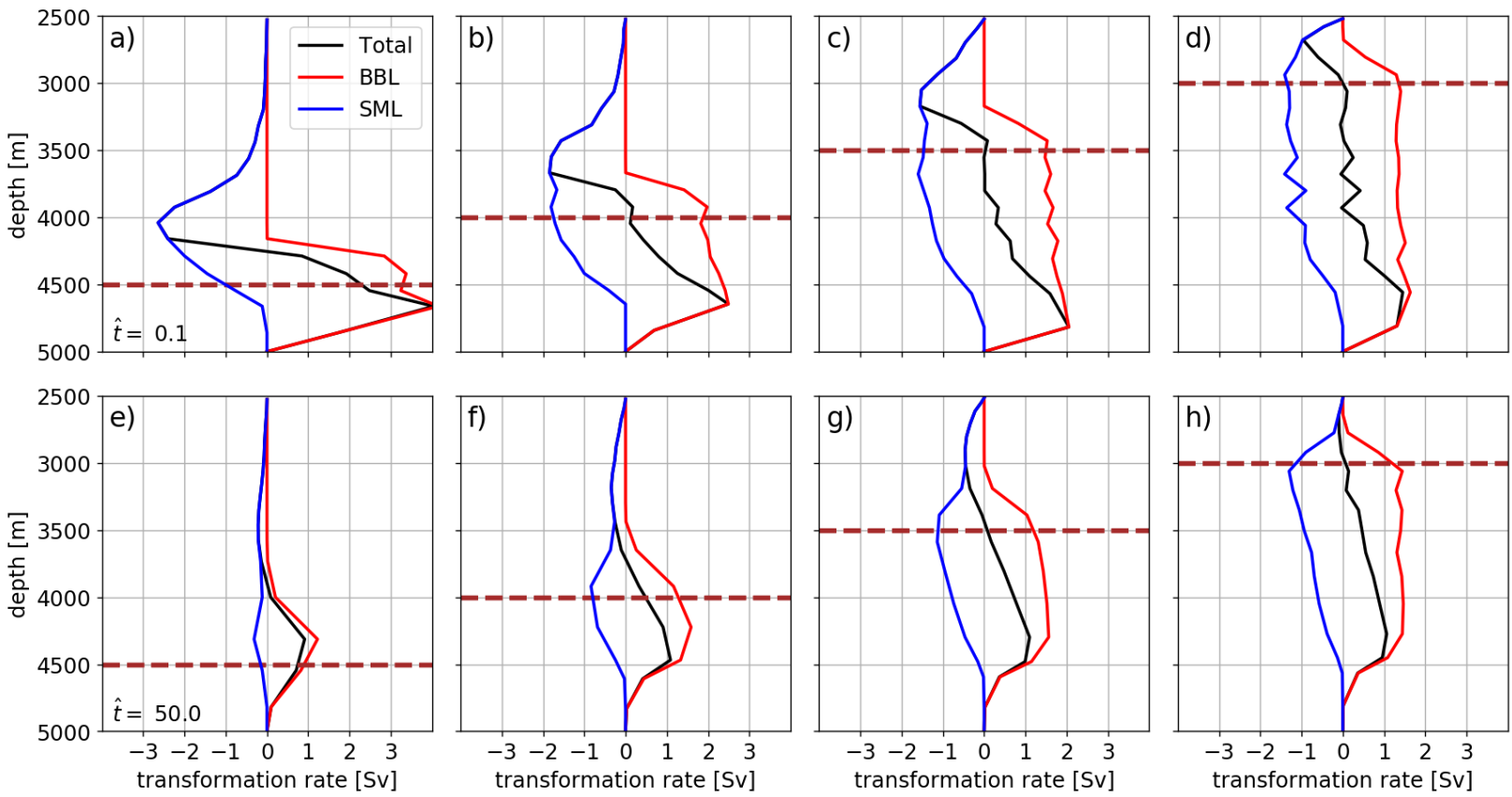

Figure 2-10: Watermass transformations in PGCM simulations as a function of ridge height. All panels are for a fixed constant stratification restoring buoyancy profile. The initial spin-up at $\hat{t}=0.1$ is shown in (a-d) and the equilibrium state at $\hat{t}=50$ is shown in (e-h). Mid-ocean ridge height increases in increments of $500 \mathrm{~m}$ from left to right, as indicated by the dashed brown lines. Black, red, and blue lines show the net, bottom boundary layer (BBL), and stratified mixing layer (SML) contributions to the watermass transformations, respectively.

\subsection{Comparison with realistic mid-ocean ridges}

The topography and mixing in the PGCM is inspired by observations from the Brazil Basin (Figure 2-11), one of the regions of the abyssal ocean best characterized by observations (e.g. St. Laurent 
et al. 2001; Thurnherr and Speer, 2003). The circulation that emerges from the PGCM-REAL simulation (Figure 2-11 b) is qualitatively similar to the circulation inferred from observations using an inverse model (Figure 2-11a, based on St. Laurent et al. 2001): bottom-enhanced mixing along the slope of the mid-ocean ridge drives upwelling in a bottom boundary layer and downwelling in a stratified mixing layer above.

To contextualize our simulated watermass transformations, we estimate watermass transforma tions in the ocean based on hydrography and a commonly-used mixing parameterization, following Ferrari et al. (2016)'s modifications of Nikurashin and Ferrari (2013) (see Cimoli et al. 2019 for the sensitivity to the results to assumptions about functional dependence of the flux coefficient and the partitioning of local vs. remote internal tide dissipation). The buoyancy flux is approxi mated by $\overline{w^{\prime} b^{\prime}}=-\Gamma \epsilon$, where $\epsilon$ is the kinetic energy dissipation and $\Gamma$ is a 'mixing efficiency' set to $\Gamma=0.2$ (Osborn, 1980); the buoyancy field (computed from the neutral density $\gamma$ ) is taken from a gridded product derived from hydrographic sections of the World Ocean Circulation Experi ment (Gouretski and Koltermann, 2004); and we impose the insulating bottom boundary condition $\mathbf{n} \cdot \overline{\mathbf{u}^{\prime} b^{\prime}} \simeq \overline{w^{\prime} b^{\prime}}=0$ (where $\mathbf{n} \simeq \mathbf{z}$ for typical bathymetric slopes of $\tan \theta \ll 1$ ). The dissipation rate $\epsilon$ is parameterized by applying linear wave radiation theory for internal tides (Nycander, 2005) and lee waves (Nikurashin and Ferrari, 2011) and assuming a fraction $q=0.3$ of the radiated en ergy is locally dissipated according to a bottom-enhanced structure function with a height scale of $500 \mathrm{~m}$ (St. Laurent and Garrett, 2002). We compare watermass transformation estimates from the ocean with estimates from PGCM-REAL, a simulation with restoring to an exponential refer ence stratification with a decay scale of $1000 \mathrm{~m}$ and which is our simulation with a stratification in the southern restoring region most similar to the Southern Ocean's (Figure 2-2 ). We focus on rectangular regions with dimensions $3000 \mathrm{~km}$ by $3000 \mathrm{~km}$ (in the $\mathrm{PGCM}$ ) or $30^{\circ}$ longitude by $30^{\circ}$ latitude (in the ocean), which encompass comparable ridge lengths and surface areas at subtropical latitudes. Watermass transformations in the PGCM-REAL simulation (Figure 2-12 a) are the result of partially compensating buoyancy flux convergence (Figure 2-12 ) in the BBL (red colors) and buoyancy flux divergence in the SML (blue colors). Qualitatively similar (but noisier) watermass transformations emerge for the mid-ocean ridge regions in the Pacific, Atlantic, and Indian (Figure $2-12 \mathrm{~b}-\mathrm{d}$, regions delineated by boxes in panels e,f). While the net transformation varies from $0.5 \mathrm{~Sv}$ in the limited-area South Pacific region to $2 \mathrm{~Sv}$ in the Indian Ocean region, the net transformation is always the result of partially compensating upwelling and downwelling. This qualitative similarity emerges in the large-scale watermass diagnostic, despite the relatively heterogeneous nature of the 

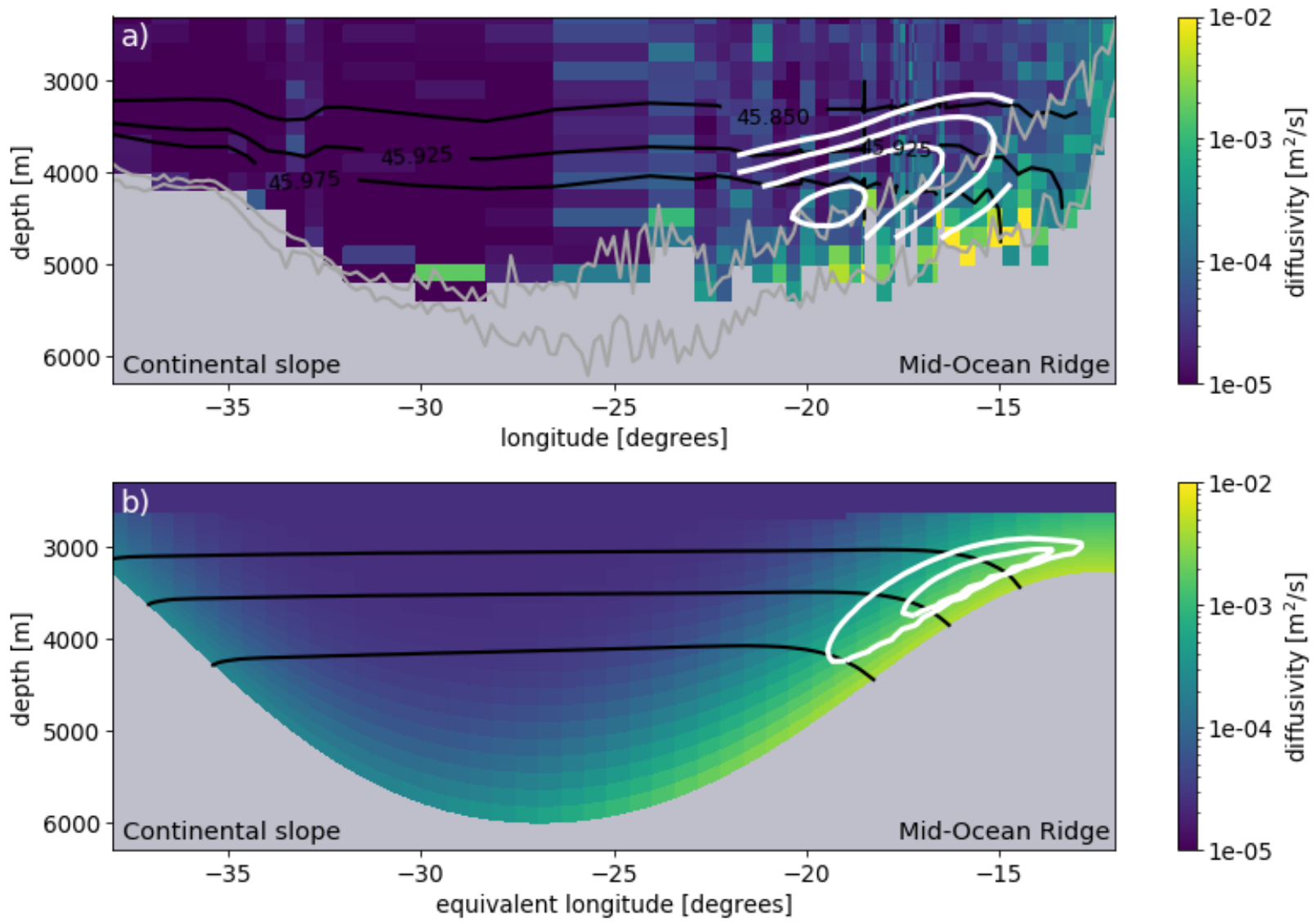

Figure 2-11: Diabatic zonal overturning circulation driven by bottom-enhanced mixing on the west ern flank of a mid-ocean ridge in (a) the South Atlantic Ocean and (b) the western half of the PGCM-REAL simulation domain. White lines show arbitrariy chosen contours of the counter clockwise zonal overturning streamfunction, where the values for (a) are digitized from Figure 14 of St. Laurent et al. (2001) and for (b) are diagnosed from PGCM-REAL simulation. Coloring shows the vertical diffusivity in log-scale (light-grey shading represents depths with no microstructure mea surements and does not necessarily represent topography), where panel (a) is inspired by Figure 2 of Polzin et al. (1997) and the diffusivity is calculated with microstructure profiles from the BBTRE experiment (Polzin et al. 1997, St. Laurent et al. 2001, archived at microstructure.ucsd.edu). Black lines are: (a) potential density $\sigma_{4}$ surfaces (referenced to $4000 \mathrm{~m}$ ) from the microstructure profiles and (b) buoyancy surfaces from the PGCM solution, chosen arbitrarily to show that the zonal over turning circulation is indeed diabatic. The dark grey lines in (a) show the depth minimum (canyon thalweg) and maximum (canyon crest) seafloor depth within $0.5^{\circ}$ latitude of the microstructure pro files. In (b), zonal distance along the PGCM section has been converted to an equivalent longitude at $25^{\circ} \mathrm{S}$ so that length scales can be directly compared between the two panels.

estimated buoyancy flux and topography in the ocean basins (compare Figure 2-12; e), because in all cases the turbulent buoyancy flux is bottom-enhanced (driving downwelling) and tapers to zero over the last grid cell to meet the insulating boundary condition within some bottom boundary layer (driving upwelling). This property of compensating watermass transformations is in contrast 
to the case of a constant buoyancy flux (Ferrari et al., 2016; Holmes et al., 2018), in which there is no compensating downwelling. Estimates of global abyssal watermass transformations, however, exhibit stronger compensation by downwelling in the SML than shown here for mid-ocean ridge regions (by factors of 2 and 3 for Ferrari et al. 2016 and McDougall and Ferrari 2017, respectively). In Section 2.8 we present evidence in support of McDougall and Ferrari (2017)'s speculation that much of this discrepancy arises due to the effects of correlations between the buoyancy flux and the stratification, which are omitted in their calculations. Ferrari et al. (2016)'s estimate includes these correlation terms but relies on poorly-sampled knowledge of the buoyancy flux and stratification close to the seafloor, which likely introduces substantial uncertainty in their estimate. While much work has gone into understanding how the compensation factor depends on various parameters of the diagnostic approach based on climatological observations and parameterized mixing (McDougall and Ferrari, 2017; Holmes et al., 2018, Cimoli et al., 2019), the functional dependence of the com pensation factor in the prognostic dynamic approach has received comparably little attention and is not well known.

\subsection{Classic recipes and new trends in abyssal cuisine}

Quantitative study of the abyssal stratification began with the classic study of Munk (1966): a pointwise theory in which the observed abyssal stratification is the result of a balance between uniform upwelling and a uniform turbulent vertical mixing. As anticipated by Munk (1966), subsequent observations show turbulent mixing to be strongly heterogeneous, with an emerging pattern of weak background mixing and vigorous mixing near rough topography (Polzin et al., 1997; Waterhouse et al. 2014). In light of these observations, Munk and Wunsch (1998) revisited Munk (1966)'s theorized point-wise vertical balance and re-derive it as a horizontally-averaged buoyancy budget, which we transcribe as

$$
\langle w\rangle A \simeq\left\langle N^{2}\right\rangle^{-1} \frac{d}{d z}\left[A(z)\langle\kappa\rangle\left\langle N^{2}\right\rangle\right]
$$

in our notation, where the key assumption is that correlations between the turbulent diffusivity $\kappa$, the stratification $N^{2}$, and the vertical velocity $w$ are all assumed to be negligible, such that $\left\langle w N^{2}\right\rangle=\langle w\rangle\left\langle N^{2}\right\rangle$ and $\left\langle\kappa N^{2}\right\rangle=\langle\kappa\rangle\left\langle N^{2}\right\rangle$. In Figure 2-13a,b,c, we show, respectively, the three terms in eq. 2.24 the horizontally-averaged stratification $\left\langle N^{2}\right\rangle$, the turbulent buoyancy flux $\langle\kappa\rangle\left\langle N^{2}\right\rangle$, and the isobath surface area (ocean area at a fixed depth) $A(z)$. In Figure 2-13d we show the leftand right-hand sides of eq. 2.24 in the PGCM-REAL simulation at equilibrium. The horizontally 

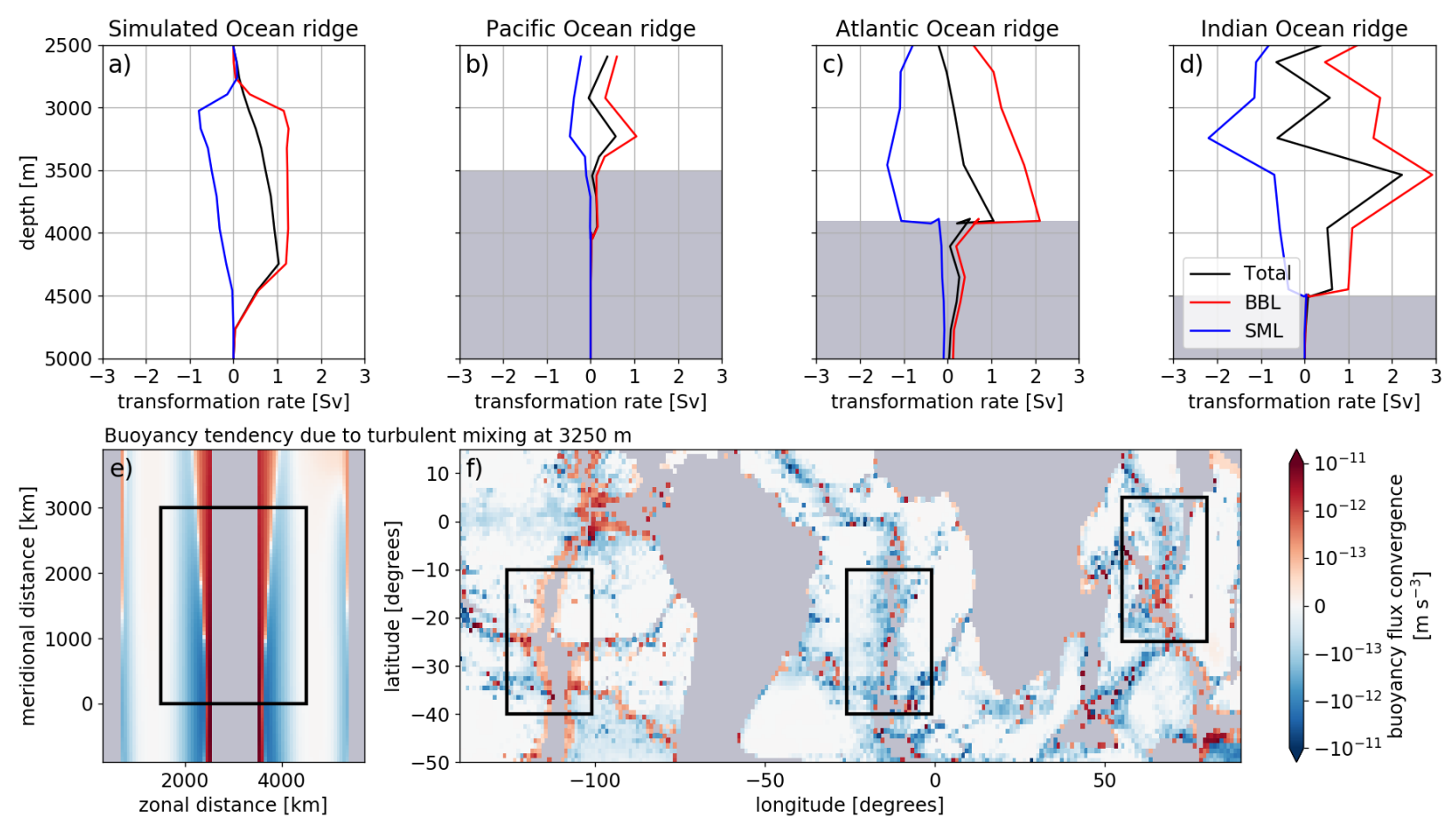

Figure 2-12: (a-d) Watermass transformations at all abyssal depths and (e,f) buoyancy flux con vergence at $3250 \mathrm{~m}$ depth in similarly-sized domains containing mid-ocean ridges, as diagnosed from (a,e) the PGCM-REAL simulation and estimated for the (b,f) Pacific, (c,f) Atlantic, and $(\mathrm{d}, \mathrm{f})$ Indian Oceans. In (a-d), the black, red, and blue lines show the net, bottom boundary layer $(\mathrm{BBL})$, and stratified mixing layer (SML) contributions to the watermass transformations, respec tively (grey shaded indicates depths representing very little ocean volume). The black boxes in $(\mathrm{e}, \mathrm{f})$ delineate the similarly-sized regions (each with dimensions of roughly $3000 \mathrm{~km} \times 3000 \mathrm{~km}$ ) for which we compute the watermass transformations. In (e,f), red and blue show regions of buoyancy flux convergence (positive buoyancy tendency) and buoyancy flux divergence (negative buoyancy tendency), respectively.

averaged vertical flux divergence (right-hand side of eq. 2.24) is a poor prediction for the diagnosed vertical transport. This is not surprising, given that 1) $w, N^{2}$, and $\kappa$ are spatially-correlated in our solutions and 2) that density surfaces are strongly sloping near boundaries. Analysis in buoyancy coordinates, such as either the thickness-weighted average framework (De Szoeke and Bennett, 1993 , Young, 2011) or the watermass transformation framework (Walin, 1982), are more appropriate. The mixing-driven watermass transformation (solid black line) equals the diapycnal transport (the diabatic MOC of interest here), by definition, but also serves as a better approximation of the vertical transport $\langle w\rangle A$ than the right hand side of eq. 2.24 .

In Figure 2-13, we show that ignoring correlations within the buoyancy flux $\left\langle\kappa N^{2}\right\rangle \approx\langle\kappa\rangle\left\langle N^{2}\right\rangle$ introduces large biases relative to the full horizontal-mean buoyancy flux, which results in even larger biases in the flux divergence (Figure $2-13 \mathrm{~d}$ ). To investigate the role of these spatial correlations 

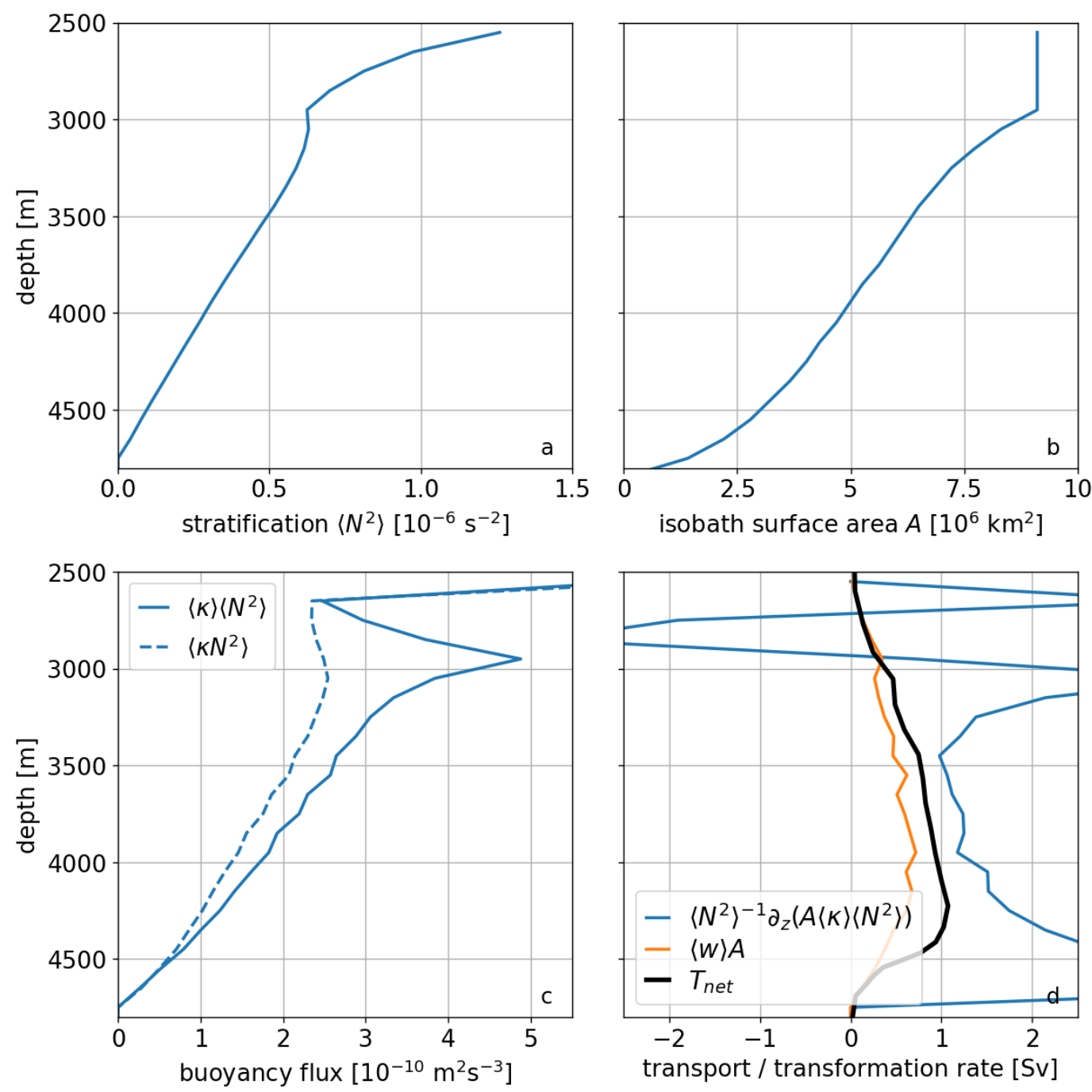

Figure 2-13: Comparison of abyssal upwelling diagnostics in the PGCM-REAL simulation for the watermass transformation analysis region highlighted in Figure 2-2 a. The orange and blue lines in panel (d) represent the left- and right-hand sides, respectively, of the horizontally-averaged advection-diffusion balance $\langle w\rangle A \simeq\left\langle N^{2}\right\rangle^{-1} \frac{d}{d z}\left[A(z)\langle\kappa\rangle\left\langle N^{2}\right\rangle\right]$ (eq. 2.24), which accounts for changes in isobath surface area $A(z)$ with depth but ignores correlations between $w, \kappa$, and $N^{2}$ and excludes the horizontal advection. The solid blue lines in panels (a), (b), and (c) show the individual compo nents of the expressions: (a) the horizontally-averaged stratification $\left\langle N^{2}\right\rangle$, (b) the isobath surface area $A(z)$, and (c) the horizontally-averaged buoyancy flux $\langle\kappa\rangle\left\langle N^{2}\right\rangle$. The dashed line in (b) shows the role of correlation terms $\left\langle\kappa N^{2}\right\rangle-\langle\kappa\rangle\left\langle N^{2}\right\rangle$ in setting the vertical structure of the buoyancy flux. Finally, the solid black line in (d) shows the net watermass transformation, where its native density coordinate has been mapped into a pseudo-depth coordinate by taking the average depth of a given buoyancy surface (eq. 2.19p. For all of our simulations, vertical advection-diffusion bulk models are poor approximations of diapycnal abyssal upwelling.

between $\kappa$ and $N^{2}$ more exactly, we return to the watermass transformation framework, where we now define $\langle\cdot\rangle \stackrel{\text { def }}{\equiv} A_{b}^{-1} \int_{A_{b}} \cdot \mathrm{d} A$ as the average along a buoyancy surface. We can thus decompose the 
vertical component of the watermass transformation into uncorrelated and correlated components, respectively:

$$
T_{\text {net }} \approx \partial_{b}\left(A\left\langle\kappa \partial_{z} b\right\rangle\right)=\partial_{b}\left(A\langle\kappa\rangle\left\langle\partial_{z} b\right\rangle\right)+\partial_{b}\left(A\left\langle\kappa^{\prime} \partial_{z} b^{\prime}\right\rangle\right)
$$

where $\kappa^{\prime}=\kappa-\langle\kappa\rangle$ and $\partial_{z} b^{\prime}=\partial_{z} b-\left\langle\partial_{z} b\right\rangle$ are deviations from the mean along a buoyancy surface. Figures 2-14a,c show that ignoring the correlation terms in the watermass transformation results in an overestimation of the net transformation by $20 \%$ to $200 \%$ because the stratification $\partial_{z} b^{\prime}$ is locally reduced in the abyssal mixing layers where $\kappa^{\prime}$ is high (orange lines in Figure 2-14a,c), with the magnitude of this bias varying dramatically across simulations with different topographic geometries and restoring profiles.

To support our hypothesis that vertical variations in the stratification are necessary to support large net watermass transformations, we further decompose the uncorrelated component into a component related to the change in the mean stratification and a residual component related to changes in both the area of the buoyancy surface and the mean diffusivity, respectively:

$$
\partial_{b}\left(A\langle\kappa\rangle\left\langle\partial_{z} b\right\rangle\right)=A\langle\kappa\rangle \partial_{b}\left\langle\partial_{z} b\right\rangle+\left(\partial_{z} b\right) \partial_{b}(A\langle\kappa\rangle)
$$

In the experiment shown in Figure 2-14a,b, where the uncorrelated component is a reasonable approximation of the net transformation, we find the net transformation to be largely driven by variations of the stratification with buoyancy, $A\langle\kappa\rangle \partial_{b}\left\langle\partial_{z} b\right\rangle$, although variations in the diffusivity integrated along a buoyancy surface are also important. Variations in the stratification also appear important in the experiment shown in Figure 2-14d, although this decomposition is more difficult to interpret since the uncorrelated component overestimates the net transformation by a factor of 3 (Figure 2-14c).

\subsection{Discussion}

The idealized numerical model presented here describes an abyssal circulation and stratification controlled by mixing-driven flows along a mid-ocean ridge in a cross-equatorial basin (Figure 2-1). By initializing with- and restoring to- a series of reference buoyancy profiles in the south of the basin, we investigate transient and equilibrium coupling between the basin stratification and the mixing-driven boundary flows. At equilibrium, dense abyssal waters form in the southern restoring region and flow north via adiabatic deep western boundary currents (red circle), filling the abyssal 

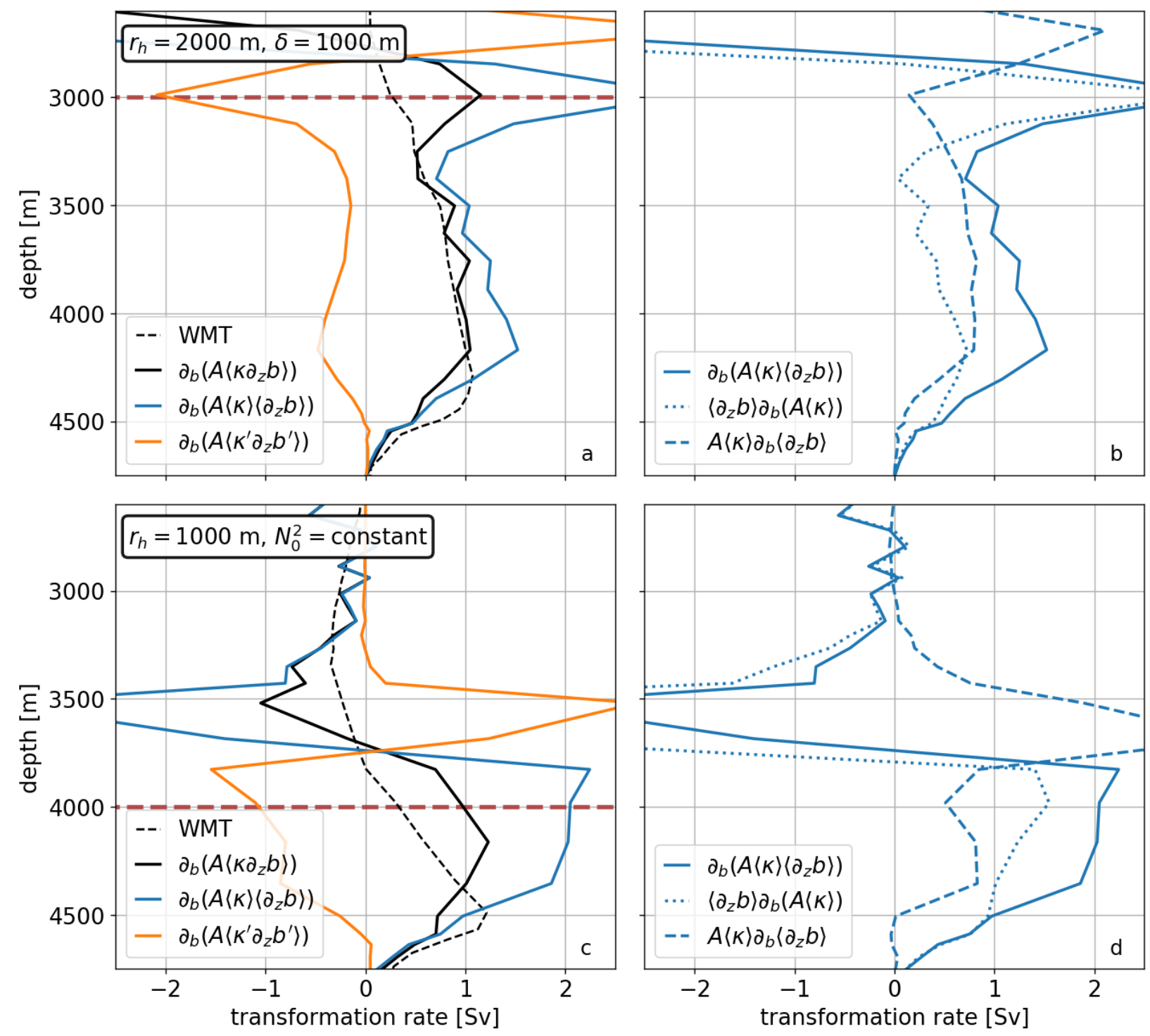

Figure 2-14: Decomposition of the watermass transformation into various physical components in the PGCM-REAL experiment (top row) and an experiment with a shorter ridge and constant reference stratification (bottom row). In panels (a,c), we approximate the full watermass transformation (dashed black) by the contribution from the vertical buoyancy flux $\partial_{b}\left(A\left\langle\kappa \partial_{z} b\right\rangle\right)$ (solid black), which we then decompose into an uncorrelated component $\partial_{b}\left(A\langle\kappa\rangle\left\langle\partial_{z} b\right\rangle\right)$ (solid blue) and a residual $\partial_{b}\left(A\left\langle\kappa^{\prime} \partial_{z} b^{\prime}\right\rangle\right)$ (solid orange), where $\langle\cdot\rangle$ denotes averaging along a buoyancy surface. In panels $(\mathrm{b}, \mathrm{d})$, we further decompose the uncorrelated component into contributions due to the buoyancy derivative of the mean stratification $\partial_{b}\left\langle\partial_{z} b\right\rangle$ (dashed) and the integrated diffusivity along a buoyancy surface $\partial_{b}(A\langle\kappa\rangle)$ (dotted).

depths in both hemispheres. Along the mid-ocean ridge, bottom-enhanced mixing (squiggly lines) drives a net transformation of dense abyssal waters into lighter deep waters, the residual of partiallycompensating upwelling in a bottom boundary layer (BBL) and downwelling in a stratified mixing 
layer (SML) right above it. The newly formed light deep waters flow zonally towards the western continental slope (solid arrow; reminiscent of Spall 2001/s two-layer model), returning southward via an adiabatic deep western boundary current to the restoring region (blue circles), and closing the abyssal overturning circulation as they are once again transformed into dense abyssal waters.

Despite the extreme degree of idealization in our formulation of the Planetary Geostrophic Circulation Model (PGCM), the watermass transformations that emerge at equilibrium are quali tatively similar to diagnostic estimates of watermass transformations near mid-ocean ridges in the Pacific, Atlantic, and Indian Oceans (Figure 2-12), which are themselves fairly uncertain (Cimoli et al., 2019; de Lavergne et al., 2016c). Similarly, the zonal overturning that emerges within bot tom mixing-driven flows along the mid-ocean ridge are qualitatively similar to that described by an inverse model of the abyssal Brazil Basin based on in-situ measurements (St. Laurent et al., 2001, and Figure 11). Remaining differences between our simulations and observations are likely due to the crude nature of our parameterizations for the restratification by submesoscale turbulence (and other unresolved effects on the stratification, such as topography blocking/steering; see Chapter 3) and for the formation of bottom waters in the Southern Ocean, as well as uncertainties in the observational estimates.

The equilibrium interior stratification in the PGCM always exhibits dynamically significant vertical variations, the structure of which is determined by a combination of mixing layer dynamics and the restoring condition in the south. Even in our simulations that are initialized from- and restored to- a constant stratification reference buoyancy profile, heterogeneities in the topographic slope cause cross-slope divergence and a corresponding exchange flow between the abyssal mixing layers and the interior. Over time, these exchange flows modify the interior stratification and associated watermass transformations.

As in CF18, we find the prediction of upwelling in the BBL by one-dimensional (1D) boundary layer theory provides a reasonable approximation to the maximum net transformation or, equiva lently, the strength of the diabatic meridional overturning circulation (Figures 2-8 and 2-9). While this interpretation provides a useful prediction for the maximum net transformation which occurs at the base of topographic slopes, it does not inform the net transformation along the flanks of the mid-ocean ridge, where upwelling in the BBL is instead partially compensated by downwelling in a SML. At depths where both the BBL and the SML are active, 1D theory predicts almost per fect compensation and a resulting net transformation that is vanishingly small (eq. 2.5 and Figure 2-8a,e). In contrast, our PGCM simulations exhibit finite net watermass transformations that ex 
tend from the base of the ridge slopes all the way up to the ridge crest (Figure 2-10), consistent with both our oceanic estimates (Figure 2-12) and inverse models of the Indo-Pacific overturning circulation (Lumpkin and Speer, 2007). We attribute the existence of a finite net transformation to vertical variations in the basin stratification 7 (Figures 2-8 and 2-14). As we increase the degree to which the restoring stratification varies in the vertical, the compensation of BBL upwelling by SML downwelling (evaluated near the depth of the ridge crest) ranges from nearly-perfect compensation to nearly-zero compensation (Figure 2-9 a). Thus, while 1D bottom boundary layer theory provides a reasonable approximation to maximum net watermass transformation, the vertical extent and structure of watermass transformations depends on the degree of compensation by downwelling in the SML, which is itself coupled to the vertically-varying basin stratification.

Callies (2018) show that 1D boundary layer models with realistic inputs produce weak nearboundary stratification and correspondingly weak BBL upwelling transports. In both the 3D PGCM and its 1D emulator, this problem has been abstracted away by tuning the simple linear momentum drag term to effectively produce a BBL stratification consistent with observations. While we justify our parameter choice a posteriori in terms of a crude parameterization for slumping of isopycnals by finite-amplitude submesoscale instabilities (proposed by Callies 2018 as a key restratification process), this should be considered little more than a placeholder until these restratification pro cesses are better understood. Thus, disentangling the processes responsible for maintaining the stratification of abyssal mixing layers over rough topography is the focus of Chapter 3 .

Our simulations show that correlations between mixing and stratification (Figure 2-14a,c), which are typically ignored in idealized models of the zonal-mean abyssal overturning (Nikurashin and Vallis, 2011; Jansen and Nadeau, 2019), can be of leading-order importance in abyssal watermass transformations; whether these correlations are important in the ocean remains an open question. Despite our improved understanding of the roles of bottom mixing and the interior basin stratifica tion on the abyssal watermass transformations and circulation, we fall short of a predictive analytical theory for the abyssal overturning and stratification that couples boundary layer dynamics with a model for the evolution of the interior stratification. Recent and ongoing work in 1) observing abyssal mixing layers (e.g. Garabato et al., 2019), 2) investigating their dynamics with idealized theory and simulations (Wenegrat et al., 2018; Callies, 2018, Holmes et al., 2019), 3) developing and evaluating parameterizations of their turbulent fluxes (Yankovsky et al., 2020), 4) and coupling

\footnotetext{
${ }^{7}$ Since the publication of this article, this basin-scale result has been confirmed by a regional study of mixingdriven watermass transformations in the Orkney Passage (Spingys et al., 2021), which finds a mixing-driven diapycnal upwelling of $0.5 \mathrm{~Sv}$ is almost entirely due to to the increase of the average stratification with buoyancy (or height).
} 
them to the basin stratification (e.g. refining the approach of Salmun et al. 1991; see my preliminary attempt in Appendix A will all be key ingredients for cooking up a revised theory of the abyssal circulation and stratification. 


\section{Chapter 3}

\section{Dynamics of eddying abyssal mixing layers over rough topography}

\subsection{Introduction}

Below the oceanic pycnocline, the vast volumes of the deep ocean are ventilated by two intercon nected cells of a global meridional overturning circulation (Gordon, 1986). The lower cell of this circulation is sourced along the coast of Antarctica, where atmospheric cooling and brine rejec tion transform surface waters into the dense Antarctic Bottom Waters (AABW) that fill the global abyssal ocean at a rate of approximately $30 \mathrm{~Sv}\left(1 \mathrm{~Sv} \stackrel{\text { def }}{\equiv} 10^{6} \mathrm{~m}^{3} / \mathrm{s}\right)$ (Talley, 2013a). Since the buoy ancy surface bounding AABW from above does not outcrop elsewhere in the ocean, conservation of mass implies that an equal amount of AABW must upwell across buoyancy surfaces (diabatically) in the abyss. Isolated from surface exchange with the atmosphere, waters below about $2000 \mathrm{~m}$ depth (corresponding to the crests of major topographic features, such as mid-ocean ridges) can only be upwelled diabatically by interior watermass transformations (e.g. small-scale turbulent mixing) or fluxes across the seafloor boundary (geothermal heating) (Walin, 1982, Tziperman, 1986; Ferrari, 2014 ).

These basic inferences of a global diabatic upwelling in the abyss (e.g. Sverdrup et al., 1942) are also consistent with more detailed inverse modelling at regional scales (e.g. Talley et al., 2003). Most notably, Hogg et al. (1982) consider the fate of $4 \mathrm{~Sv}$ of AABW (colder than $0{ }^{\circ} \mathrm{C}$ ) that enters the Brazil Basin from the Southern Ocean through the Vema Channel; since there are no other exits from the basin and since geothermal fluxes are relatively weak, they infer a basin-averaged turbulent diffusivity of $\mathcal{O}\left(3 \mathrm{~cm}^{2} / \mathrm{s}\right)$ is required to upwell these waters across the $0{ }^{\circ} \mathrm{C}$ isotherm. 
Early measurements of in-situ turbulence in the upper $\sim 1000 \mathrm{~m}$ of the interior ocean find diffusivities more than an order of magnitude smaller than those predicted by the large-scale abyssal tracer budgets described above (Gregg, 1987; Ledwell et al., 1993). A subsequent field campaign in the abyssal waters of the Brazil Basin finds similarly weak background diffusivities over the smooth topography of the abyssal plains, but reveals diffusivities that increase downwards by several orders of magnitude over the rough topography of the Mid-Atlantic Ridge (Polzin et al., 1997, Ledwell et al., 2000). Huang and Jin (2002) simulate how this observed bottom-enhanced mixing modifies the classical theories of basin-scale abyssal circulation: bottom-enhanced mixing drives interior downwelling as opposed to upwelling (Munk, 1966) and the basin-scale horizontal circulation (Stommel, 1958) is instead dominated by narrow mixing-driven flows along the ridge flanks.

The bottom-enhancement of mixing over rough topography, confirmed by many subsequent measurements (Waterhouse et al. 2014), is thought to be largely due to the local breaking of internal waves generated by the sloshing of the barotropic tide over rough abyssal topography St. Laurent et al. 2001; Nikurashin and Legg, 2011). Theoretical understanding of internal-wave generation, propagation, and breaking processes has enabled the development of increasingly sophisticated internal wave mixing parameterizations (St. Laurent and Garrett, 2002; Polzin, 2009; Melet et al., 2014 Lavergne et al., 2020), which have been used to extrapolate mixing estimates globally.

Combined with hydrographic measurements of the buoyancy field, these global maps of param eterized mixing rates provide the first quantitative estimates of global abyssal watermass transfor mations (Nikurashin and Ferrari, 2013; de Lavergne et al., 2016c). Based on these estimates, and inspired by the pioneering studies of mixing in the Brazil Basin, Ferrari et al. (2016) and McDougall and Ferrari (2017) develop a revised conceptual model of the global mixing-driven abyssal upwelling: mixing-driven diabatic upwelling is confined to a thin Bottom Boundary Layer (BBL) just above insulating (or geothermally heated) seafloor while bottom-enhanced mixing drives diabatic downwelling in the Stratified Mixing Layer (SML) above; the net diabatic overturning is the residual of these two opposing mixing layer flows. In this emerging framework, the global overturning cir culation is modulated by the dynamics of thin BBLs; however, since these abyssal boundary layer flows are challenging to observe (Garabato et al., 2019; Spingys et al., 2021) and are too small to be resolved by the coarse abyssal vertical resolution in conventional general circulation models (Callies and Ferrari, 2018b), they remain poorly understood (Callies, 2018; Wenegrat et al., 2018; Drake et al., 2020a).

The interpretation of the role of boundary mixing in the abyssal overturning circulation has a 
contentious history: on the one hand, in-situ observations of weakly-stratified BBLs would seem to imply the existence of vigorous boundary mixing (Armi, 1978); on the other hand, mixing of already well-mixed water is inefficient ${ }^{1}$ and does not lead to significant watermass transformation (Garrett, 1979). These two arguments are based on two fundamentally different views about the restratification processes that sustain the stratification and mixing at equilibrium:

1. an observation-based three-dimensional (3D) perspective in which vigorous mixing creates transient well-mixed BBLs; a finite near-bottom stratification is maintained by exchange with the well-stratified SML due to topographic separation or baroclinic eddies, as evidenced by weakly-stratified step-like layers carrying suspended sediment and other anomalies into the interior (Armi, 1977, Armi and D'Asaro, 1980, Kunze et al., 2012, McPhee-Shaw et al., 2021)

2. a theory-based one-dimensional (1D) perspective in which a buoyancy-driven up-slope flow maintains the near-bottom stratification (Garrett, 1990, 1991, 2001).

The two views are not inherently contradictory: even when the flow may be described as $1 \mathrm{D}$ at leading order, cross-slope variations in the stratification (Salmun et al., 1991), topography (Dell and Pratt, 2015), and mixing rates (Kunze et al. 2012) may drive slope-normal exchanges between the interior and the boundary at higher order (McDougall, 1989, Garrett et al., 1993).

Sloping boundary layer theory, by virtue of its simplicity and generality, provides a useful ground ing for the study of mixing layer flows. The key idea is to approach the problem from a coordinate frame aligned with the mean topographic slope and to assume uniformity along the plane of the slope, aside from a constantly stratified and hydrostatic background state. Wunsch (1970) de rives one-dimensional solutions in which slope-normal mixing causes buoyancy convergence at the seafloor boundary and the resulting buoyancy force drives up-slope flow. Thorpe (1987) extends the theory to account for rotation, and shows that an along-slope thermal wind shear develops in response to horizontal buoyancy gradients, which drives a corresponding Ekman-like modification to the cross-slope boundary layer flow. Using the same model, Garrett (1990) clarifies the role of the buoyancy-driven up-slope "secondary" circulation in maintaining a finite near-bottom equilib rium stratification upon which mixing can efficiently act. In light of Toole et al. (1994)'s pioneering

\footnotetext{
${ }^{1}$ Throughout the thesis, we take the perspective that turbulent buoyancy fluxes can be parameterized as a downgradient vertical diffusive flux $-\kappa b_{z}$. In this framing, we assume the diffusivity increases towards the bottom and the no-flux boundary condition on buoyancy is instead satisfied by vanishing of the stratification; alternatively, this condition can be implicit in the diffusivity profile, as in Holmes and McDougall (2020). Analogously, in dissipationbased closures of the buoyancy flux $\Gamma \epsilon$, it is instead typically assumed that the flux coefficient $\Gamma$ vanishes near the boundary; in recent parameterizations, for example, this is achieved by a decrease of $\Gamma$ with the buoyancy Reynolds number $R e_{b}=\frac{\epsilon}{\nu b_{z}}$ at high $R e_{b}$ or, equivalently, a linear scaling $\Gamma \propto b_{z}$ (Melet et al. 2013, Mashayek et al. 2017b).
} 
observations, Thompson and Johnson (1996) explore transient numerical solutions for the case of bottom-enhanced mixing and present qualitative evidence of their relevance to the Pacific Ocean abyssal circulation.

In light of diagnostic evidence for boundary-control on the abyssal circulation (Ferrari et al., 2016), Callies (2018) tests whether sloping BBL theory is quantitatively consistent with observa tions. In his analysis of the sloping flank of the Mid-Atlantic Ridge in the Brazil Basin (where co-located measurements of both abyssal mixing rates and stratification are available), he finds that the steady-state 1D boundary layer solution forced by the observed mixing exhibits a stratification an order of magnitude weaker than observed. Thus, the watermass transformations sustained by the 1D "secondary" circulation are too inefficient to contribute significantly to the global abyssal over turning circulation. In Chapter 2, we used a simple Rayleigh drag parameterization as a placeholder for the yet unknown physical processes that maintain a realistically high mixing layer stratification; here, we tackle this restratification problem head on.

To reconcile boundary layer dynamics with observations, Callies (2018) argues the stratification of abyssal mixing layers is instead maintained by submesoscale baroclinic eddies, which act to slump sloping buoyancy surfaces back to the horizontal. Indeed, Wenegrat et al. (2018) demonstrate that mixing-driven 1D boundary layers solutions are linearly unstable to submesoscale baroclinic modes, in a manner similar to the well-studied analagous problem in the surface mixed layer (Boccaletti et al. 2007). Callies (2018) simulates the finite amplitude evolution of these instabilities in a 3D generalization of the 1D boundary layer framework and show that the solutions converge on a quasi-equilibrium stratification consistent with observations.

While Callies (2018)'s 3D solutions for a smoothly sloping plane appear consistent with avail able observations of the stratification, this is a relatively weak constraint; as will become clear later on, different combinations of various dynamical processes can produce similar stratifications. As discussed by Callies (2018), it is not clear to what extent such idealized solutions are directly applicable to the mid-ocean ridge, which is famously characterized by particularly rough topogra phy. For example, any of the observations of bottom-enhanced mixing, stratification, and diabatic upwelling from the region are confined to $\mathcal{O}(500 \mathrm{~m})$-deep fracture zone canyons which cut across the ridge (Polzin et al., 1997, Ledwell et al., 2000; St. Laurent et al., 2001; Thurnherr et al., 2020). To account for these leading-order topographic features, Ruan and Callies (2020) run simulations of mixing-driven flow over a sinusoidal mid-ocean ridge incised by an idealized Gaussian fracture zone canyon. They find that the canyon sidewalls suppress along-slope (or cross-canyon) flow and 
thus support a vigorous up-canyon (or cross-slope) mean flow; the restratifying tendency of this up-canyon mean flow is much stronger than that of either the 1D up-slope flow or the submesoscale eddies on the smooth ridge flanks, implying that abyssal watermass transformations are, per unit area, much larger within the canyons than on the ridge flanks. However, they acknowledge that a major caveat of their results is that the simulated stratification in the canyon is orders of magnitude larger than is observed, suggesting they are still missing important physics. In addition to frac ture zone canyons, mid-ocean ridges are also characterized by smaller-scale anisotropic abyssal hills; these features have characteristic scales taller than 1D BBLs and comparable to those of the fastest growing baroclinic mode (Wenegrat et al., 2018), so we would expect them to affect both mean and eddying circulations. Within the fracture zone canyons, abyssal hills often manifest as sills that substantially block or constrain the up-slope flow, leading to hydraulic acceleration of mean flows and locally enhanced turbulence (Thurnherr et al., 2005, Dell, 2013; Dell and Pratt, 2015; Clément et al., 2017). The complexity induced by the boundary conditions at a rough seafloor prevents the direct application of existing idealized theories to in-situ observations (see also Polzin, 2009), as one might do for the surface mixed layer. This complexity is exacerbated by the sparsity of observa tional constraints (de Lavergne et al., 2016a); as a result, our understanding of abyssal mixing layer dynamics remains comparatively poor.

Here, we use a quasi-realistic "slope-native" numerical simulation to bridge the gap between idealized 1D BBLs and the complexity of observed flows in a region scarred by a fracture zone canyon and dotted with abyssal hills. In Section 3.2, we review key insights from the 1D BBL buoyancy budget and derive a generalized buoyancy budget that permits topographic variations and spatio-temporal eddy correlations. In Section 3.3. we describe the simulation configuration, which leverages a coordinate frame aligned with the mean topographic slope to apply doublyperiodic boundary conditions that allows mean up-slope flow across background buoyancy gradient to support a non-trivial equilibrium solution. In Section 3.4, we describe the simulated mixing layer flows and show they are qualitatively consistent with available observations. In Section 3.5 . we analyze the simulated buoyancy budget, and show a three-way balance between 1) bottomenhanced mixing, 2) cross-slope mean flow, and a 3) slope-normal eddy flux. By progressively simplifying the configuration in a hierarchy of models framework (Held, 2005), we quantify the role of particular physical processes in setting the near-boundary stratification; in the process, we reproduce the qualitative results of all of the idealized simulations discussed above. In Section 3.6, we explore the magnitude and structure of mixing-driven watermass transformations in the BBL 
and SML. In Section 3.7, we discuss how our results bridge the gap between in-situ observations Armi, 1978, Thurnherr and Speer, 2003, Polzin, 2009) and 1D BBL theory (Garrett, 1979), and how they illustrate - at a regional scale- the control of abyssal mixing layers on an "upside-down" abyssal overturning circulation (Ferrari et al., 2016). We conclude that a combination of both submesoscale eddies and topographically-controlled secondary circulations are required to maintain a steady-state near-boundary stratification consistent with in-situ observations and a finite abyssal overturning circulation.

\subsection{Theory}

\subsubsection{Slope-native equations}

In sloping boundary layer theory (e.g. Wunsch, 1970, Garrett et al., 1993, Callies, 2018), analytical progress is achieved by modelling the system in a more natural coordinate frame aligned with its mean topographic slope, rather than the typical coordinate frame $(\hat{x}, \hat{y}, \hat{z})$ with $\hat{z}$ aligned with gravity. It is useful to decompose the buoyancy $b=B+b^{\prime}$ into a background component $B \stackrel{\text { def }}{\equiv} N^{2} \hat{z}$, where $N^{2}$ is a constant vertical buoyancy gradient, and a perturbation component $b^{\prime}(\hat{x}, \hat{y}, \hat{z}, t)$; the background buoyancy is assumed to be in hydrostatic balance with a background pressure, $\frac{\mathrm{d} P}{\mathrm{~d} \tilde{z}}=B$, and we similarly decompose $p=P+p^{\prime}$. Then, we rotate the coordinate system from the gravity-aligned frame to the coordinate frame aligned with the mean-slope $(x, y, z) \stackrel{\text { def }}{\equiv}(\hat{x} \cos \theta+$ $\hat{z} \sin \theta, \hat{y}, \hat{z} \cos \theta-\hat{x} \sin \theta$ ), where $\theta$ is the region's average slope angle in the $\hat{x}$-direction (e.g. dashed black lines in Figure $3-2 \mathrm{~b}$ ). For small slopes $\tan \theta \ll 1$, the hydrostatic Boussinesq equations in the mean-slope coordinates are, at leading order, given by ${ }^{2}$

$$
\begin{gathered}
u_{t}+\mathbf{u} \cdot \nabla u-f v=-p_{x}^{\prime}+b^{\prime} \sin \theta+\nabla \cdot(\nu \nabla u), \\
v_{t}+\mathbf{u} \cdot \nabla v+f u=-p_{y}^{\prime}+\nabla \cdot(\nu \nabla v), \\
p_{z}^{\prime}=b^{\prime} \cos \theta \\
\nabla \cdot \mathbf{u}=0 \\
b_{t}^{\prime}+\mathbf{u} \cdot \nabla b^{\prime}+N^{2}(w \cos \theta+u \sin \theta)=\nabla \cdot\left[\kappa\left(N^{2} \cos \theta \mathbf{z}+\nabla b^{\prime}\right)\right],
\end{gathered}
$$

where subscripts represent partial derivatives, $\nabla$ is the gradient operator, $u$ is the along-canyon (or cross-slope) velocity, $v$ is the cross-canyon (or along-slope) velocity, $w$ is the slope-normal velocity,

\footnotetext{
${ }^{2}$ While $\sin \theta \simeq \theta$ and $\cos \theta \simeq 1$ in this limit, we retain these geometric terms explicitly so they are not forgotten.
} 
$f$ is a constant Coriolis parameter ( $f$-plane approximation), $\kappa$ is an isotropic eddy diffusivity, and $\nu=\sigma \kappa$ is an isotropic eddy viscosity determined by the turbulent Prandtl number $\sigma$. The alongcanyon $\mathbf{x}$-momentum equation is identical in form to the zonal $\hat{\mathbf{x}}$-momentum equation with the exception of the small but dynamically significant projection of the perturbation buoyancy force $b^{\prime} \hat{\mathbf{z}}$ on $\mathbf{x}$. Advection and diffusion across the background buoyancy gradient effectively enter as forcing terms in the perturbation buoyancy equation.

The anomalous seafloor depth, relative to the mean slope, is given by

$$
d(x, y)=\hat{d}(\hat{x}, \hat{y})+\hat{x} \tan \theta
$$

We set $z=0$ along the sloping plane that intersects the point with the greatest anomalous seafloor depth, $\max (d)$. Boundary conditions at the seafloor, $z=\max (d)-d(x, y)$, are $\mathbf{u}=0$ (no-slip and no-normal-flow) and $\mathbf{n} \cdot(\kappa \nabla b)=0$ (insulating), where $\mathbf{n}$ is a unit vector normal to the boundary.

\subsubsection{Smooth planar slopes and steady 1D dynamics}

Assuming a smoothly sloping infinite plane $(d \equiv 0)$ and mixing rates that vary only in the slopenormal direction, the equilibrium solution also varies only in the slope-normal direction and the equations dramatically reduce down to

$$
\begin{gathered}
-f v=b^{\prime} \sin \theta+\partial_{z}\left(\nu u_{z}\right), \\
f u=\partial_{z}\left(\nu v_{z}\right), \\
p_{z}^{\prime}=b^{\prime} \cos \theta \\
u N^{2} \sin \theta=\partial_{z}\left[\kappa\left(N^{2} \cos \theta+b_{z}^{\prime}\right)\right],
\end{gathered}
$$

where the continuity equation $w_{z}=0$ combines with the no-normal-flow bottom boundary condition at $z=0$ to require $w \equiv 0$ everywhere (no slope-normal exchange). These equations can be solved analytically in the case of constant parameter values (Wunsch, 1970; Phillips et al., 1986, Garrett, 1990), or approximately in the case for varying parameters in some asymptotic limits (Salmun et al. 1991; Callies, 2018). In either case, the slope Burger number $S \stackrel{\text { def }}{\equiv} N^{2} \sin ^{2} \theta / f^{2}$ and the BBL thickness

$$
\delta \stackrel{\text { def }}{\equiv} q^{-1}=\sqrt{\frac{2 \nu}{f}}(1+S \sigma)^{-\frac{1}{4}}
$$


emerge as key parameters; we recognize $\delta$ as the Ekman layer thickness $\delta_{E} \stackrel{\text { def }}{=} \sqrt{\frac{2 \nu}{f}}$ modified by buoyancy effects at the sloping boundary; for typical abyssal values, $S \ll 1$ and buoyancy effects are weak.

We briefly review key results from sloping BBL theory; we closely follow the modern presentation of Callies (2018), who uses boundary layer analysis to derive insightful approximate analytical solutions to the steady 1D system (eqs. 3.7 3.10 forced by exponentially bottom-enhanced mixing, $\kappa(z ; d)=\kappa_{\mathrm{BG}}+\kappa_{\mathrm{BOT}} e^{-(z-d) / h}$ with $\kappa_{\mathrm{BOT}} / \kappa_{\mathrm{BG}} \gg 1$. These approximate solutions are valid in the limit that the inner BBL thickness $\delta$ is much thinner than the decay scale $h$ of the diffusivity, i.e. $h / \delta \gg 1$, as is the case for the rough mid-ocean ridge flanks characterized by a small slope Burger number (see Polzin 2009 for a theoretical parameterization of the decay-scale based on heuristic internal wave dynamics). For transient solutions or equilibrium solutions for which $h / \delta \ngtr 1$, we present the results of numerical solutions instead, also following the methods of Callies (2018).

Under the crucial assumption of a constant background vertical stratification $N^{2}$, the slopenative buoyancy equation (3.5) reduces to (3.10), a direct balance between slope-normal diffusion of heat downwards towards the boundary and cross-slope advection against the constant background buoyancy gradient; this balance is a near-boundary analog of Munk (1966)'s classic interior ocean vertical balance. It is also useful to integrate 3.10 in the slope-normal direction,

$$
\psi(z) \stackrel{\text { def }}{\equiv} \int_{0}^{z} u \mathrm{~d} z=\kappa \cot \theta\left(b_{z} / N^{2} \cos \theta\right)^{\beth}=\kappa \cot \theta\left(1+b_{z}^{\prime} / N^{2} \cos \theta\right)
$$

where we define $\psi$ as the up-slope transport (per along-slope unit length) and we have invoked the insulating bottom boundary condition on the full stratification, $b_{z}=0$ at $z=0$. Equation (3.12) reveals two keys insights:

1. The net up-slope transport, integrated over both the upwelling BBL and the downwelling SML, converges to the negligibly small value

$$
\psi_{\infty} \stackrel{\text { def }}{\equiv} \psi(z \rightarrow \infty)=\kappa_{\mathrm{BG}} \cot \theta \quad \text { (the } 1 \mathrm{D} \text { integral constraint) }
$$

since far from the boundary $b_{z}^{\prime} \rightarrow 0$ and $\kappa \rightarrow \kappa_{\mathrm{BG}}$ (Thorpe, 1987).

2. Maximal up-slope transport in the BBL is achieved when both $\kappa$ is large (i.e. near the boundary) and $b_{z}$ is large (strong restratification). If the stratification is maintained near the large background value $N^{2} \cos \theta$ where the diffusivity is largest (i.e. for $z \ll h$ ) then the up 
slope transport in the $\mathrm{BBL}$ reaches an upper $\operatorname{limit}]^{3} \max \{\psi\} \simeq \kappa_{\mathrm{BOT}} \cot \theta=\frac{\kappa_{\mathrm{BOT}}}{\kappa_{\mathrm{BG}}} \psi_{\infty} \gg \psi_{\infty}$.

In Callies (2018)'s solution, the equilibrium stratification $b_{z}$ is approximately inversely proportional to $\kappa$ in the SML (their eq. 10; Figure 3-8a, solid lines), such that the turbulent buoyancy flux $\kappa b_{z}$ is constant and finite buoyancy flux convergence occurs only within the thin BBL. Since the near-boundary stratification is reduced to roughly $b_{z} \approx \frac{\kappa_{\mathrm{BG}}}{\kappa_{\mathrm{BOT}}} N^{2} \cos \theta$ (Figure 3-8a, solid lines) and near-boundary mixing is thus inefficient, up-slope BBL transport is roughly equal to the negligibly small integral constraint (3.13), $\max \{\psi\} \simeq \kappa_{\mathrm{BG}} \cot \theta$ (Figure 3-7a, dotted and dashed lines). This weak BBL upwelling and irrelevant SML downwelling contrasts with the strong bi-directional flows inferred from watermass transformation analyses (Ferrari et al., 2016; McDougall and Ferrari, 2017).

Callies (2018) proposes a simple parameterization of restratification by $3 \mathrm{D}$ submesoscale baro clinic eddies as a way to improve the $1 \mathrm{D}$ boundary layer solution such that it can maintain a realistically-large near-bottom stratification. The main effect of baroclinic eddies is to extract avail able potential energy from the mean flow by slumping sloping buoyancy surfaces back towards the horizontal; this adiabatic process is most conventionally parameterized as an eddy overturning cir culation (Gent and McWilliams, 1990; Fox-Kemper et al., 2008). Taking advantage of thermal wind balance $\left(f v_{\hat{z}}=b_{\hat{x}}\right)$, the slumping of isopycnals by baroclinic instability- which decreases horizontal buoyancy gradients $b_{\hat{x}}$ - can equivalently be parameterized as a reduction in the vertical shear $v_{\hat{z}}$, e.g. by enhanced vertical momentum diffusion (Rhines and Young, 1982, Greatbatch and Lamb, 1990, Young, 2011). Since vertical momentum diffusion is the simplest parameterization to imple ment and interpret, we follow Callies (2018) in parameterizing submesoscale eddy restratification by artificially increasing the vertical eddy viscosity $\nu=\sigma \kappa$, setting $\sigma=230$ as a best fit to the observed stratification. This parameterization results in weakening the slope-normal shear of the along-slope flow and, because of the approximate thermal wind balance $f v_{z} \simeq b_{z}^{\prime} \sin \theta$ that holds in the SML (eq. 3.8), results in a corresponding weakening of the negative perturbation stratification $b_{z}^{\prime}$ (equivalent to a strengthening of the total stratification $b_{z}$; compare dash-dotted and solid lines in Figure 3-8a). At equilibrium, this parameterized eddy restratification triples $b_{z}, \kappa b_{z}$, and $\psi$ at the top of the 1D solution's BBL (Figure 3-7b, dotted lines), bringing the watermass transformations of the 1D BBL more in line with the global overturning (Callies, 2018).

\footnotetext{
${ }^{3}$ While one can imagine scenarios in which the stratification could be locally much larger than the background value, we only find small overshoots $(<20 \%)$ across all of our experiments.
} 


\subsubsection{Rough topography and eddy fluxes}

We aim to derive a generalized version of the 1D steady buoyancy budgets $3.10,3.12$ for slop ing boundary layers that allows for topographic and flow variations along the plane of the slope. Consider the buoyancy budget for a volume $\mathcal{V}$ within a height $z$ above the mean slope:

$$
\iiint_{\mathcal{V}\left(z^{\prime}<z\right)} b_{t} \mathrm{~d} V=\iint_{\mathcal{A} \stackrel{\text { def }}{=} \partial \mathcal{V}\left(z^{\prime}<z\right)}(-\mathbf{u} b+\kappa \nabla b) \cdot \mathbf{n} \mathrm{d} A
$$

where we use the divergence theorem to rewrite the right-hand side terms in terms of fluxes normal to the bounding surface $\partial \mathcal{V}$ (Figure 3-1 $)$. Fluxes through the seafloor at $z^{\prime}=\max d-d(x, y)$ vanish due to the no-flow and insulating bottom boundary conditions. Motivated by the simulations in Section 3.3. we presume fluxes through cross-slope and along-slope boundaries cancel due to periodicity (e.g. $b^{\prime}(x)=b^{\prime}\left(x+L_{x}\right)$ ), except for the up-slope flow across the background buoyancy gradient $\left(\right.$ recall $\left.b=b^{\prime}+N^{2} \hat{z}\right)$,

$$
\iint_{\mathcal{A}\left(x+L_{x} ; z^{\prime} \leq z\right)}(-u b) \mathrm{d} y \mathrm{~d} z^{\prime}-\iint_{\mathcal{A}\left(x ; z^{\prime} \leq z\right)}(-u b) \mathrm{d} y \mathrm{~d} z^{\prime}=-N^{2} L_{x} \sin \theta \iint_{\mathcal{A}\left(x ; z^{\prime} \leq z\right)} u \mathrm{~d} y \mathrm{~d} z^{\prime}
$$

This, combined with the slope-normal component of the flux through the $z^{\prime}=z$ surface, gives

$$
\underbrace{\iiint_{\mathcal{V}\left(z^{\prime} \leq z\right)} b_{t} \mathrm{~d} V}_{\text {LHS }}=\underbrace{\underbrace{\left\langle\kappa b_{z}\right\rangle}_{\text {Mixing }} \underbrace{-\langle w b\rangle}_{\text {Eddies }} \underbrace{-N^{2} L_{x} \sin \theta \Psi}_{\text {Mean Flow }}}_{\text {RHS }}
$$

where we define $\langle\cdot\rangle \stackrel{\text { def }}{\equiv} \iint_{\mathcal{A}(z)} \cdot \mathrm{d} x \mathrm{~d} y$ as the slope-integral operation and $\Psi(z) \stackrel{\text { def }}{\equiv} \iint_{\mathcal{A}\left(x ; z^{\prime} \leq z\right)} u \mathrm{~d} y \mathrm{~d} z^{\prime}$ as the up-slope transport across the periodic boundary (Figure 3-1a). At equilibrium, the form of the generalized volume-integral buoyancy equation (3.16) is similar to the 1D transport equation (3.12), although there is now an additional eddy flux of buoyancy towards or away from the boundary, and the turbulent buoyancy flux may be modified by along- and cross-slope correlations between $\kappa$ and $b_{z}$. Assuming a steady state and integrating up far enough into the interior that $\kappa \rightarrow \kappa_{\mathrm{BG}}$ and the perturbations vanish, we recover the integral constraint $(3.13)$ on the net up-slope transport from the $1 \mathrm{D}$ solution, $\Psi_{\infty} / L_{y}=\kappa_{\mathrm{BG}} \cot \theta$.

The phenomenology of idealized bottom mixed layer eddies, which are trapped within a hew hundred meters of the bottom, suggests that their buoyancy flux reaches a maximum a few meters above the bottom, implying they have a tendency to cool the BBL, warm the SML, and restratify 
(a) generalized slope-normal buoyancy budget

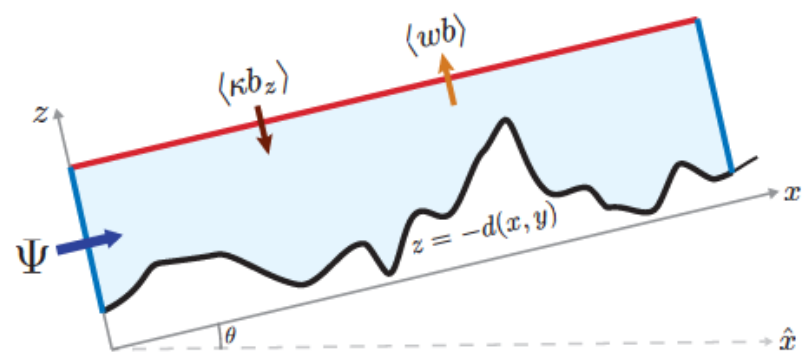

(b) height-above-bottom watermass transformations

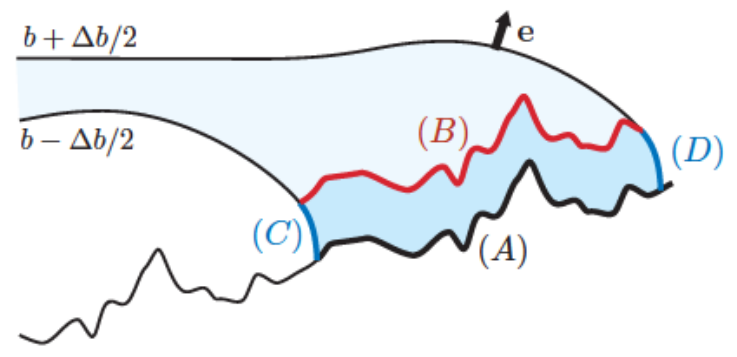

Figure 3-1: Two methods for quantifying drivers of diabatic transport in abyssal mixing layers. (a) A generalized slope-normal buoyancy budget (3.16), derived by integrating the buoyancy equation below a given height above the mean slope $z$ (volume shown in blue); at equilibrium, the upslope transport is given by $\Psi \propto\left\langle\kappa b_{z}\right\rangle-\langle w b\rangle$. (b) Watermass transformation analysis, derived by integrating the buoyancy budget along a buoyancy surface (or over a buoyancy layer, e.g. $\left\{b-\frac{\Delta b}{2}, b+\right.$ $\left.\frac{\Delta b}{2}\right\}$, colored in blue). It is useful to decompose the integrand to quantify the spatial structure of watermass transformations; for example, the watermass transformations for the thin BBL (darker blue) is given by the divergence theorem as the sum of the fluxes through its bounding surfaces: (A)-(D).

the near-boundary water (Callies, 2018; Wenegrat et al., 2018).

\subsection{Numerical model setup}

We simulate 3D mixing-driven flows using the hydrostatic Boussinesq equations in the MIT General Circulation Model (MITgcm; Marshall et al., 1997), across a hierarchy of increasingly idealized configurations. For simplicity, we assume a linear equation of state and ignore salinity variations, such that potential temperature $T$ and buoyancy $b \stackrel{\text { def }}{=} g \alpha T$ are directly proportional and are used interchangeably throughout, where $g=9.81 \mathrm{~m} / \mathrm{s}^{2}$ is the gravitational acceleration and $\alpha=2 \times 10^{-4{ }^{\circ} \mathrm{C}^{-1}}$ is a typical thermal expansion coefficient.

\subsubsection{Realistic bathymetry}

Most of the results describe a core realistic-bathymetry simulation of the Brazil Basin sub-region sampled by both the Brazil Basin Tracer Release Experiment ("BBTRE", Ledwell et al. 2000) and Dynamics of the Mid-Ocean Ridge Experiment ("DoMORE", Clément et al. 2017). We extract the Brazil Basin's seafloor topography from the Global Bathymetry and Topography at 15 Arc Sec dataset (SRTM15+; Tozer et al., 2019), which includes many more multibeam measurements than previous products (e.g. Smith and Sandwell, 1997) and thus better resolves both the BBTRE 
fracture zone canyon and the smaller-scale abyssal hills characteristic of mid-ocean ridges 4 . We interpolate the bathymetry onto a locally-tangent Cartesian grid $(\hat{x}, \hat{y}, \hat{z})$ aligned with the BBTRE Canyon, where $\hat{x}$ denotes the along-canyon dimension and $\hat{y}$ denotes the cross-canyon dimension (Figure 3-2 a), and produce a gridded bathymetry field $\hat{d}(\hat{x}, \hat{y})$. The simulated canyon stretches from a few $\mathrm{km}$ west of the Tracer Release Experiment site around $18.5^{\circ} \mathrm{W}$ (Ledwell et al., 2000) to a few $\mathrm{km}$ east of the DoMORE sill that dramatically constrains the up-canyon flow at $14.5^{\circ} \mathrm{W}$ Clément et al., 2017). Thus, our simulation domain contains several of the best-studied dynamical features of the Brazil Basin (Figure 3-2a,b).

\subsubsection{Implementing the perturbation Boussinesq equations in the mean-slope coordinate frame}

Following Section 3.2.1, we transform equations (3.1 3.5) into a coordinate frame aligned with the domain's mean slope. Equations (3.1 3.5) are solved in terms of the perturbation variables, with the background buoyancy field $N^{2} \hat{z}$ entering only indirectly via linear and inhomogeneous terms in the perturbation buoyancy equation, implemented as additional explicit tendency terms in the MITgcm equations. To stabilize the numerical solution in a way that allows submesoscale eddies to grow, we additionally implement biharmonic hyper-diffusion of momentum and buoyancy along the plane of the slope. Hyper-diffusive tendencies are sufficiently small to not alter the budgets presented here at leading order, so we omit them in all of our analyses. We enforce an insulating 5 boundary condition on the full buoyancy at the seafloor: $\mathbf{n} \cdot(\kappa \nabla b)=0$.

Relative to the mean slope, the anomalous seafloor topography $d(x, y) \stackrel{\text { def }}{\equiv} \hat{d}(\hat{x}, \hat{y})-\hat{x} \tan \theta$ is by construction nearly continuous across the periodic boundaries in the along-canyon direction $\mathbf{x}$ and in the cross-canyon direction $\mathbf{y}$; to avoid any remaining discontinuities across these boundaries due to aliasing of abyssal hills, we join the two boundaries smoothly by linear interpolation over 7 grid cells in $\mathbf{x}$ and 11 grid cells in $\mathbf{y}$, chosen such that the resulting artificial slopes do not exceed those typical of the region.

By 1) removing the constantly-stratified background state from the prognostic variables, 2) formulating the model in the slope coordinate frame, and 3) making the boundary conditions and forcing terms periodic in the $(x, y)$ plane, we are free to apply periodic boundary conditions to state

\footnotetext{
${ }^{4}$ Polzin (2009) caution that errors in bathymetric products result in mischaracterizations of mixing hotspots, e.g. whether mixing is higher on the hilly ridge flanks or along the slopes of fracture zone canyons.

${ }^{5}$ Geothermal heating is thought to contribute negligibly to abyssal watermass transformations in the BBTRE canyon region (Thurnherr et al., 2020), so we ignore it for simplicity here.
} 
a) Numerical simulation domain $\&$ points of interest

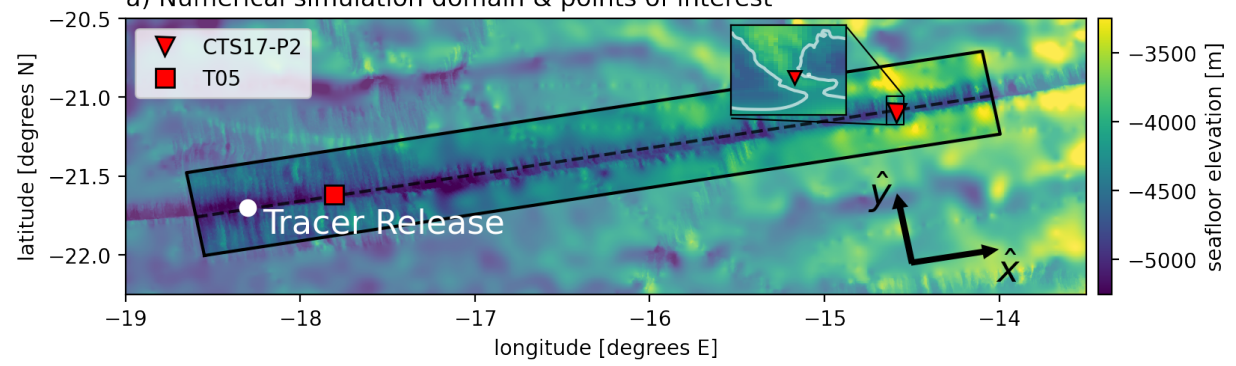

b) Prescribed bottom-enhanced mixing in a mean-slope coordinate frame
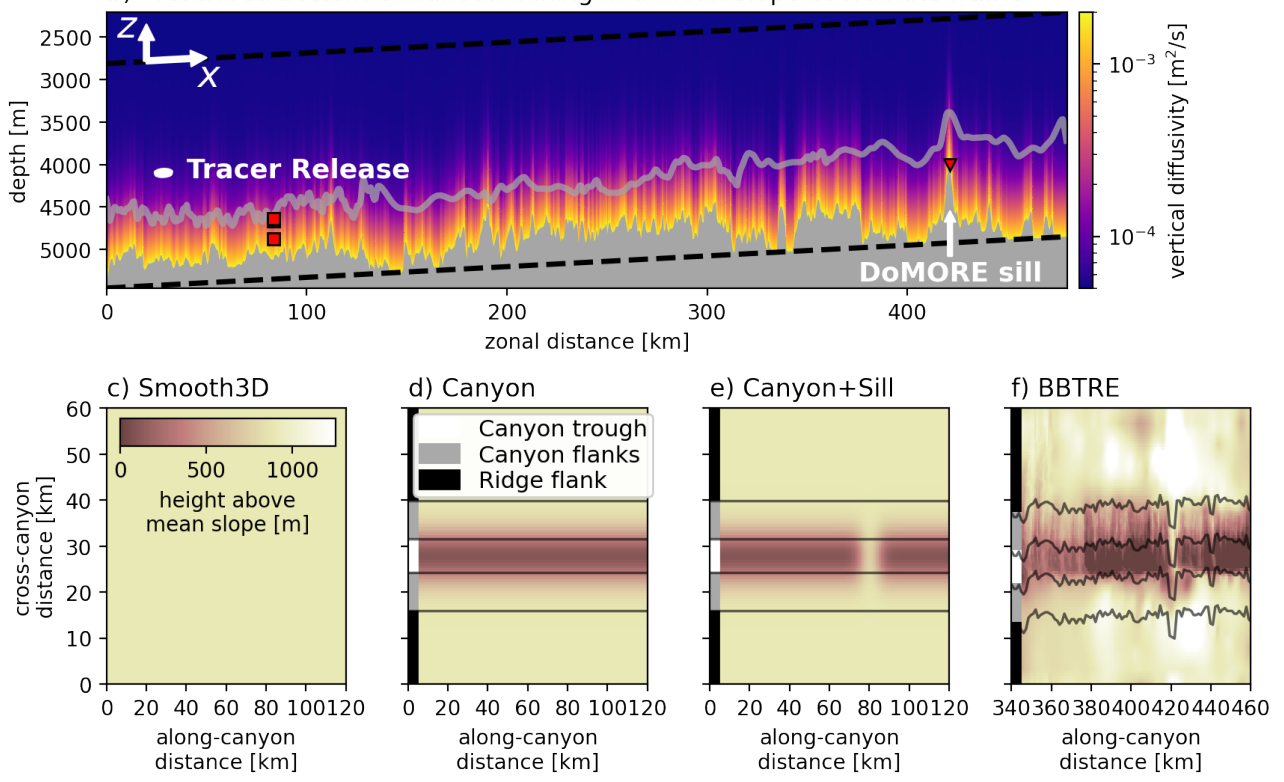

Figure 3-2: Numerical model domains. (a) Seafloor elevation $-\hat{d}(\hat{x}, \hat{y})$, including the doubly-periodic simulation domain centered on the Brazil Basin Tracer Release Experiment (BBTRE) canyon. Red markers show the locations of moorings from Clément et al. (2017) (CTS17) and Thurnherr et al. (2005) (T05). The inset highlights the DoMORE sill that dramatically constrains up-canyon flow. White markers mark the injection location from the BBTRE (Ledwell et al., 2000). (b) Imposed slope-normal diffusivity field, the result of applying a self-similar exponential profile as a function of the height-above-bottom (eq. 3.17) to variable topography. Arrows show the Cartesian along-canyon $\hat{\mathbf{y}}$ and cross-canyon $\hat{\mathbf{x}}$, as well as the transformed slope-normal $\mathbf{z}$ and along-canyon $\mathbf{x}$ coordinate vectors, which appear distorted because the vertical dimension is exaggerated. (c-f) A hierarchy of simulations with progressively complex seafloor bathymetry geometries (relative to a constant mean slope of angle $\theta$; see dashed lines in panel b). Thin black lines distinguish three sub-regions: the canyon trough, the canyon's flanks, and the ridge flank surrounding the canyon.

variables $\mathbf{u}, b^{\prime}$, and $p^{\prime}$ in both $\mathbf{x}$ and $\mathbf{y}$.

\subsubsection{Forcing by observed bottom-enhanced turbulent mixing}

Following the classic one-dimensional boundary layer configuration (Wunsch, 1970), we inject energy into the system only through small scale turbulent mixing, which is parameterized as a slope-normal 
diffusive buoyancy flux $\left(-\kappa \partial_{z} b\right) \mathbf{z}$. We use Callies (2018)'s self-similar height-above-bottom profile

$$
\kappa(x, y, z)=\kappa(z ; d)=\kappa_{\mathrm{BG}}+\kappa_{\mathrm{BOT}} \exp \left(-\frac{z+d}{h}\right)
$$

with $\kappa_{\mathrm{BOT}}=1.8 \times 10^{-3} \mathrm{~m}^{2} / \mathrm{s}, \kappa_{\mathrm{BG}}=5.3 \times 10^{-5} \mathrm{~m}^{2} / \mathrm{s}$, and $h=230 \mathrm{~m}$; these parameter values are chosen by performing a least-squares fit to the height-above-bottom-average of 126 microstructure profiles in the BBTRE region. While turbulent mixing in this region is known to be intermittent in both time and space (Clément et al., 2017), the sparsity and noisiness of individual profiles does not provide sufficient detail to formulate a parameterization with a richer spatial structure. We imagine this imposed bottom-enhanced mixing to represent a variety of turbulent ocean processes, but especially the breaking of internal waves (Whalen et al., 2020). We assume that small-scale turbulent mixing acts similarly to mix momentum, i.e. we assume a turbulent Prandtl number of $\sigma \stackrel{\text { def }}{\equiv} \frac{\nu}{\kappa}=1$.

This configuration implies a directionality to the simulated flow of energy: local mixing provides available potential energy, some of which is converted into kinetic energy by a mean flow and its instabilities, and all of which is eventually dissipated by the imposed eddy viscosity; there is no explicit external forcing by winds, tides, or non-local mean flows.

\subsubsection{Parameter regime}

Following Callies (2018), we assume a background far-field stratification $N=1.3 \times 10^{-3} \mathrm{~s}^{-1}$ and a local Coriolis parameter $f=-5.3 \times 10^{-5} \mathrm{~s}^{-1}$ characteristic of the BBTRE region above the weakly stratified BBLs. A linear fit to the bathymetry $d(x, y$,$) yields the domain's average topographic$ slope angle $\theta=1.26 \times 10^{-3}$ in $\hat{\mathbf{x}}$. Mixing layers are thus characterized by weak stratification and gentle large-scale slopes, equating to a small Burger number, $S \stackrel{\text { def }}{\equiv} N^{2} \tan ^{2} \theta / f^{2}=10^{-3} \ll 1$ and BBL thickness $\delta \approx 8 \mathrm{~m}$ (eq. 3.11).

\subsubsection{Numerics}

The horizontal grid spacing of $\Delta x=\Delta y=600 \mathrm{~m}$ is fine enough to permit the anticipated sub mesoscale baroclinic turbulence, which for the 1D sloping BBL problem has a maximum linear growth rate near the local deformation radius $L \sim \frac{N H_{M L}}{f}=6 \mathrm{~km}$ (Wenegrat et al., 2018), where $H_{M L} \approx 250 \mathrm{~m}$ is the thickness of the weakly-stratified bottom layer (Callies, 2018). Yet, the grid spacing is also coarse enough for three-dimensional modelling of the entire $480 \mathrm{~km}$ by $60 \mathrm{~km}$ region 
to be computationally feasible. We set $\kappa_{4} \equiv \nu_{4}=2 \times 10^{4} \mathrm{~m}^{4} / \mathrm{s}$, the smallest value that maintains a stable solution, so that the hyper-diffusion interferes minimally with diapycnal buoyancy fluxes and the growth of submesoscale instabilities (Callies and Ferrari, 2018b; Ruan and Callies, 2020). In the vertical, a cell thickness of $\Delta z=6 \mathrm{~m}$ (with partial cells down to $1.2 \mathrm{~m}$ ) marginally resolves the predicted $\mathcal{O}(10 \mathrm{~m})$-thick BBL. A 1D spin-up experiment with these parameters confirmed this ver tical resolution is sufficient to accurately reproduce the analytical solution for BBL upwelling (not shown). Far above the sloping sea floor, the cell thickness $\Delta z$ is stretched up to $50 \mathrm{~m}$ to efficiently fit the several-kilometer vertical scale of abyssal mixing layers (Callies, 2018) into a domain that spans a height $H=2700 \mathrm{~m}$ above the mean slope.

We spin up the simulations from a constantly-stratified rest state $\left(b^{\prime} \equiv 0, \mathbf{u} \equiv \mathbf{0}\right)$. The BBL adjusts rapidly on a timescale $\tau_{\mathrm{BBL}}=\delta^{2} / \kappa_{\mathrm{BOT}}=10$ hours. While the full equilibration of the solution occurs over a prohibitively long diffusive timescale characteristic of the abyssal ocean inte rior, $\tau_{\mathrm{INT}}=H^{2} / \kappa_{\mathrm{BG}} \approx 5000$ years, buoyancy tendencies are small enough by $t=5000$ days in the bottom $1000 \mathrm{~m}$ (see Section 3.5) that we consider the solution sufficiently quasi-equilibrated for the analyses presented here. For the initial spin-up, we use a centered second order advection scheme with as large a timestep as possible under the CFL constraint $(\Delta t=60 \mathrm{~s})$ for efficiency and accu rate conservation of tracers. At quasi-equilibrium, we switch to a higher-order non-linear advection scheme and a smaller timestep of $\Delta t=15 \mathrm{~s}$ for more stable and accurate tracer diagnostics.

\subsubsection{Hierarchy of progressively idealized simulations}

The simulations in our model hierarchy differ only in their seafloor topography, domain length, and dimensionality. We progressively idealize the BBTRE canyon configuration (Figure 3-2f): first, we remove the abyssal hills along the ridge flank and idealize the geometry of the remaining canyon and sill features ("Canyon+Sill"; Figure 3-2 ); second, we remove the sill ("Canyon"; 3-2d); third, we remove the canyon entirely ("Smooth3D"; Figure 3-2 ); and finally, we eliminate variations along the plane of the slope, collapsing the solution onto a single slope-normal dimension as in classical BBL theory ("1D"). For reference, we also include some additional variants on the 1D model where we vary one parameter at a time: non-rotating ( $1 \mathrm{D}_{f=0}$ "), non-sloping ( $\left.1 \mathrm{D}_{\theta=0} "\right)$, and parameterized eddies ("1D $\left.\mathrm{D}_{\sigma=230} "\right)$. Unless we specify otherwise, results refer to the default BBTRE simulation. 


\subsection{Mixing-driven up-canyon flow, submesoscale turbulence, and stratification}

At quasi-equilibrium, the time-mean flow (averaged over days 5000 to 5500) is dominated by a vigorous up-canyon jet along the canyon thalweg, banked along the steeper southern flank of the canyon (as in Dell, 2013, Ruan and Callies, 2020). The up-canyon jet exhibits a maximum along canyon-averaged velocity of $\bar{u}^{x}=0.75 \mathrm{~cm} / \mathrm{s}$ about $400 \mathrm{~m}$ above the seafloor (Figure 3-3a). This up-slope jet is partially compensated by a down-slope jet on the gentler northern flank, such that the cross-canyon-averaged velocity is reduced to $\bar{u}^{y}=\mathcal{O}(0.1 \mathrm{~cm} / \mathrm{s})$ (Figure 3-3a,b). The up-stream jet accelerates as it spills over two major cross-canyon sills: the BBTRE sill at $x=110 \mathrm{~km}$ and the DoMORE sill at $x=420 \mathrm{~km}$ (Figure 3-3a,b), both suggestive of hydraulic control (Pratt and White head, 2008). The vertically-integrated cross-slope transport $\int_{z=0}^{H} u \mathrm{~d} z$ is dominated by standing eddy features above the canyon (Figure 3-3, recall $z=0$ at the deepest point relative to the mean slope), but prominently features meandering up- and down-canyon jets when integration is restricted to

just the canyon itself, $\int_{z=0}^{800 \mathrm{~m}} u \mathrm{~d} z$ (Figure 3-3d). These simulated mixing-driven means flows can be compared against two in-situ mooring observations: the BBTRE mooring at $x=110 \mathrm{~km}$ just upstream of the BBTRE sill (Toole 2007; also analyzed by Thurnherr et al.2005) and the DoMORE mooring at $x=420 \mathrm{~km}$ atop the DoMORE sill (Clément et al., 2017). At the DoMORE sill, hor izontal and vertical constrictions accelerate the up-canyon flow to $5 \mathrm{~cm} / \mathrm{s}$ over a layer $\delta z=150 \mathrm{~m}$ thick and $\delta x=2.5 \mathrm{~km}$ wide (Figure 3-4a). The simulated transport at the DoMORE sill is roughly constant in time, also suggestive of hydraulic control (Pratt and Whitehead, 2008), and is about $25 \%$ that suggested by the mooring observations (half as fast and half as thick; Figure 3-4b). By contrast, at the BBTRE mooring, which is a few $\mathrm{km}$ upstream of the BBTRE sill, the up-canyon flow is much weaker $(u \approx 0.75 \mathrm{~cm} / \mathrm{s})$ but spread over a thicker $(\delta z \approx 600 \mathrm{~m})$ and wider $(\delta x \approx 5 \mathrm{~km})$ layer, such that the total up-canyon transport at the two sections are similar (Figure 3-4 ); such a comparison is impossible from observations since the single BBTRE mooring profile cannot be reasonably extrapolated across the canyon (Thurnherr et al. 2005). The simulated flow at the BBTRE mooring has roughly the same vertical structure as in the moored current meter velocities, but about half their magnitude (Figure $3-4 \mathrm{~d}$ ). The relative weakness of the simulated flows suggest that either the imposed microstructure-based mixing rates are too biased low (Thurnherr et al. 2005, as hypothesized by) or that we have omitted important physics, such as tidal rectification effects or large-scale external forces. 

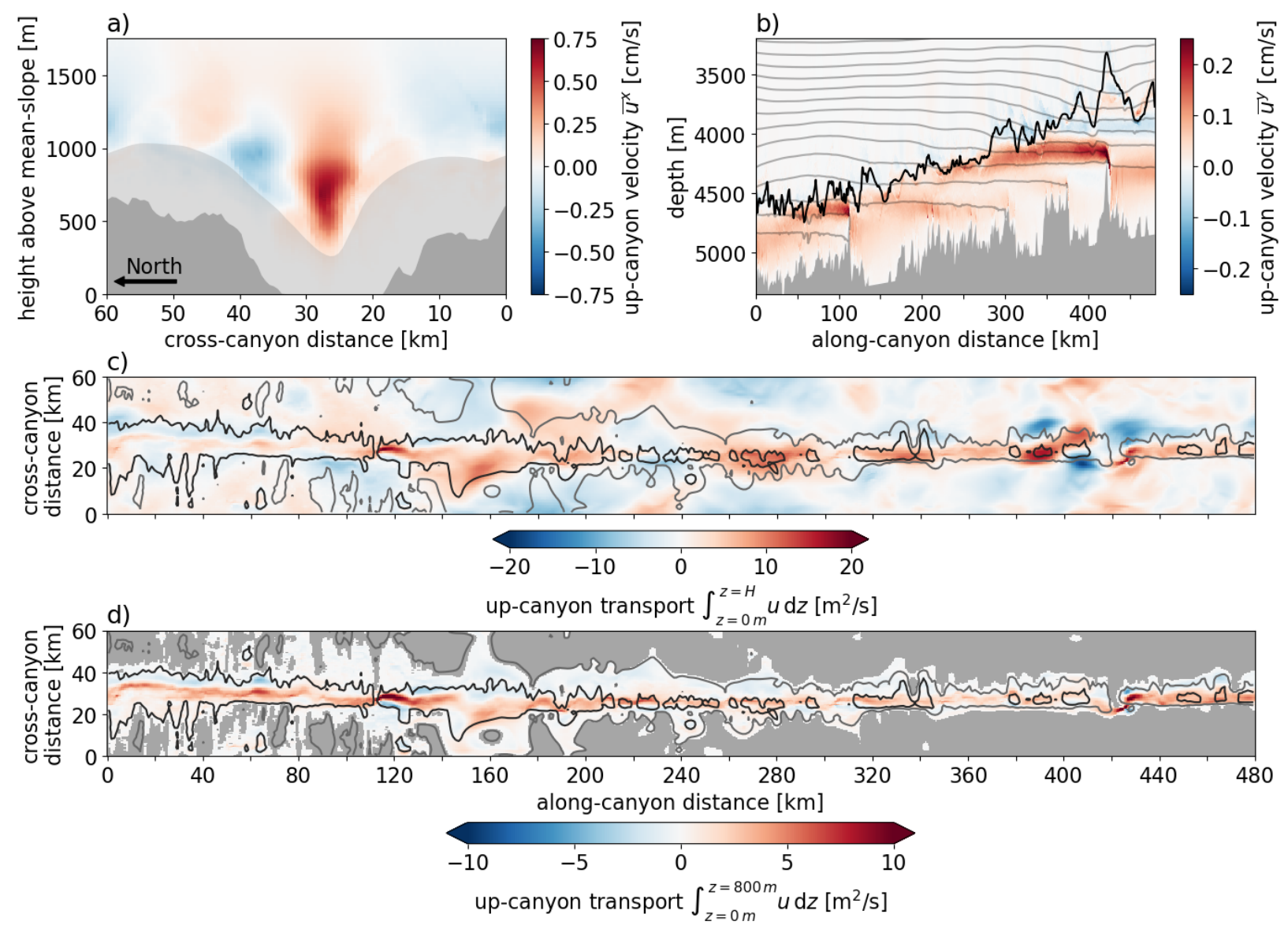

Figure 3-3: Structure of up-canyon mean flow in the BBTRE Canyon. (a) Along-canyon-averaged up-canyon flow $\bar{u}^{x}$, with the mean canyon seafloor outlined in transparent grey shading and crosscanyon thalweg shown in the dark gray shading. (b) Cross-canyon-averaged up-canyon flow $\bar{u}^{y}$ in the Cartesian coordinate frame $(\hat{x}, \hat{z})$. Grey lines represent equally-spaced buoyancy surfaces, with dashed lines showing the full buoyancy field (800 grid column profiles) and solid lines showing the buoyancy field subsampled like the hydrographic observations (11 equally-spaced profiles Thurnherr et al., 2020), which gives the misleading appearance of buoyancy surfaces gently impinging into the bottom. The black line marks the mean seafloor depth of the half of the domain furthest from the canyon thalweg and acts as a proxy for the crest of the canyon. (c) Up-canyon flux, integrated in the slope-normal direction $z$. Black and grey contours show a deep and shallow isobath, respectively, to highlight the canyon topography that shallows to the right. (d) Same as (c), but integrated only from $z=0 \mathrm{~m}$ to $z=800 \mathrm{~m}$ to highlight the core up-canyon jet within the canyon.

Averaging the BBTRE simulation in height-above-bottom (hab) coordinates reveals that the stratification generally remains close to its background value, except in the $\mathcal{O}(10 \mathrm{~m})$-thick BBL (Figure 3-5a, solid blue line). Upon first inspection, this result appears inconsistent with observa tions which, when averaged in hab, exhibit much weaker stratification up to $1 \mathrm{~km}$ above the seafloor (Figure 3-5, red lines). However, much of this discrepancy is resolved by sampling the simulation at the exact locations of the observational profiles (Figure 3-5b), and comparing their sample-mean 

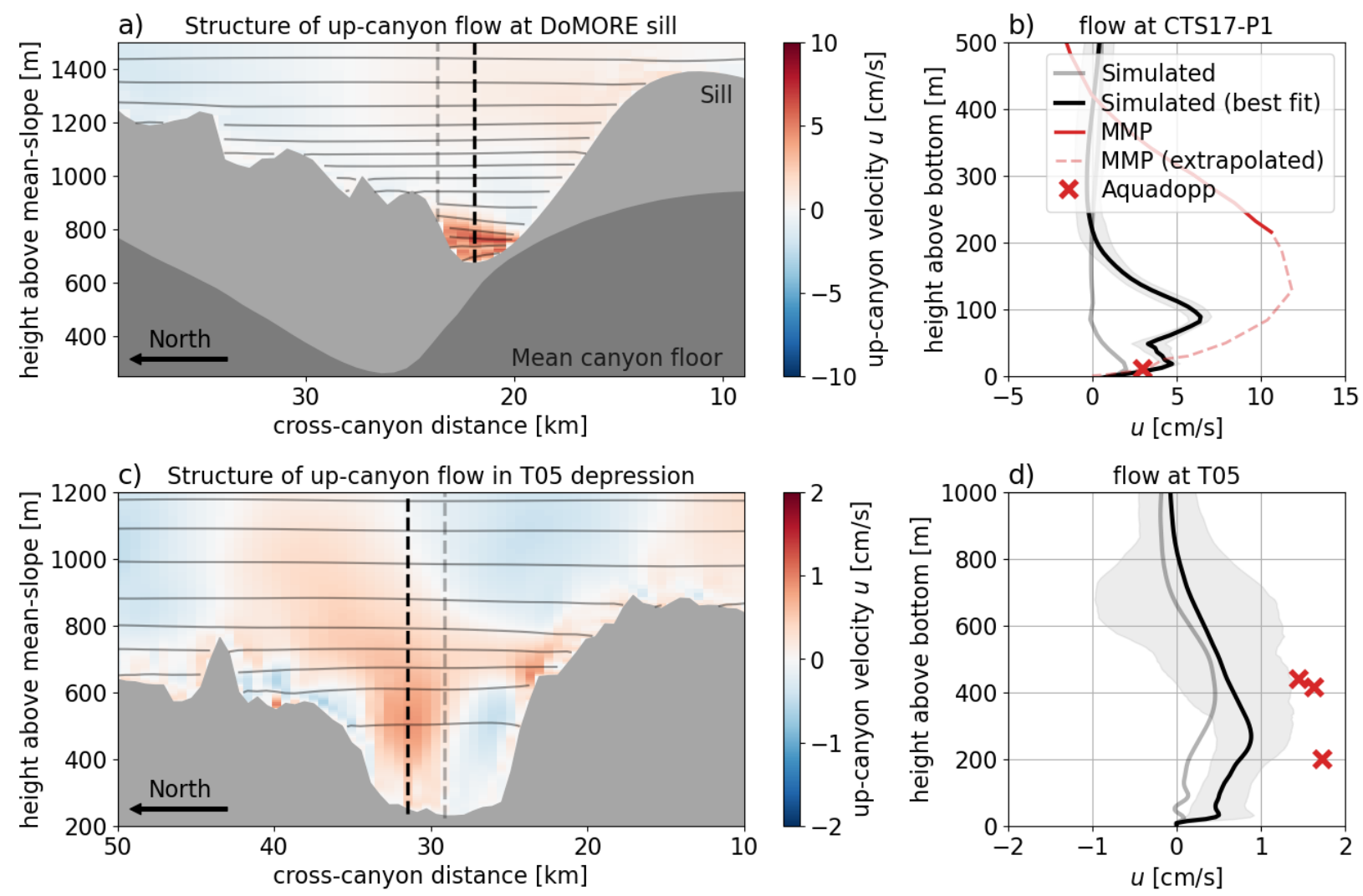

Figure 3-4: Structure of up-canyon flow at two mooring sites. (a,c) Cross-canyon sections of the upcanyon flow at locations of DoMORE sill mooring (Clément et al., 2017) (CTS17-P1) and BBTRE mooring Thurnherr et al. (2005) (T05). Light grey shading shows the local seafloor depth while the dark grey shading in (a) shows the mean height of the canyon floor above the mean slope, highlighting the significant vertical and cross-canyon constriction introduced by the sill. (b,d) Height-above-bottom profiles of the up-canyon flow at the locations of the two moorings (light grey lines) and shifted a few grid columns over to improve the fit.

to that of the observations (Figure 3-5a). Since the BBTRE sampling strategy was to find as much tracer as possible, the field campaign specifically focused on sampling the deep depressions in the BBTRE canyon, which appear to exhibit unusually weak stratification compared to the canyon flanks, sills, and the surrounding ridge flank. However, even with this like comparison, the simula tion is still too stratified by about a factor of two relative to the observations; we attribute this to 1) the possibility that the turbulent mixing we prescribe is too weak, 2) residual buoyancy tendencies, 3) the omission of large-scale vertical gradients in the background stratification $N^{2}$ (see Chapter 2), or 4) other unknown effects. We discuss these points further in Section 3.7 .

The time-mean view of the up-canyon circulation above betrays a rich field of submesoscale eddies which have radii comparable to the deformation radius and are trapped within a few hundred meters of the seafloor, including within the $\mathcal{O}(10 \mathrm{~km})$-wide canyon (Figure 3-6). These eddies 

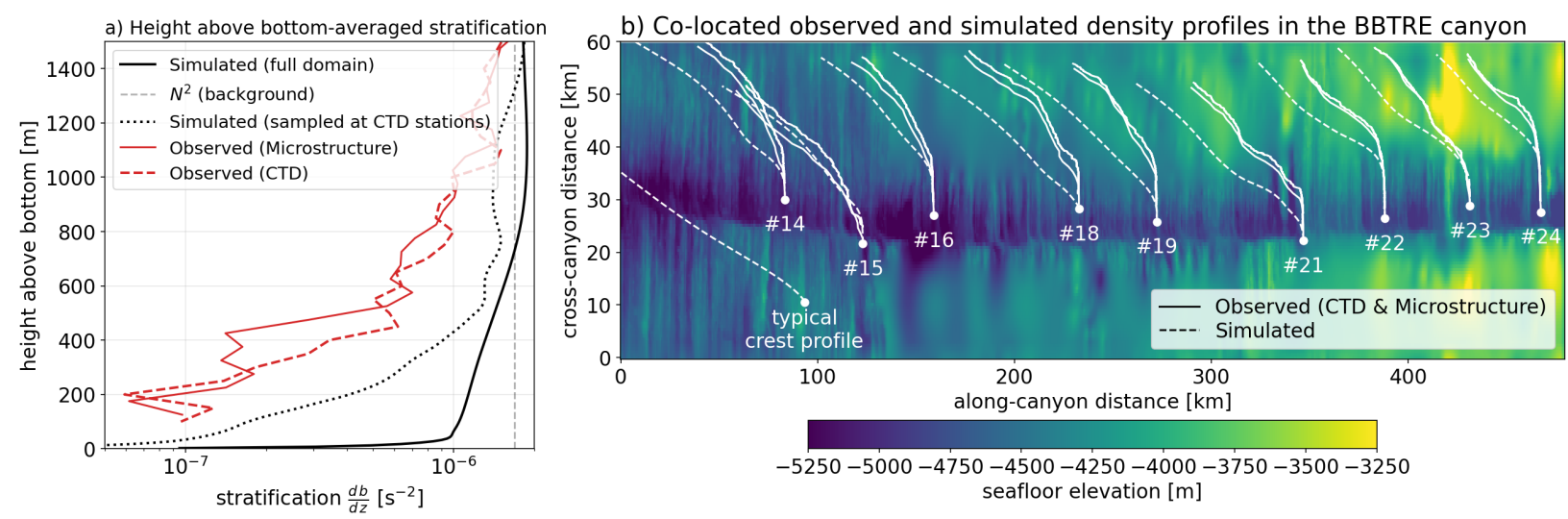

Figure 3-5: Comparison between observed and simulated stratification in the BBTRE Canyon re gion. (a) Height above bottom-averaged profile of stratification for the full simulation domain (solid black), for the sample-mean of nine co-located CTD casts (dashed red) and free-falling microstruc ture profilers (solid red), and for the sample-mean of nine simulated profiles sampled at the locations of the observations (dotted black). (b) Observed (solid) and simulated (dashed) stratification pro files at the nine locations sampled by the BBTRE observational campaign, overlaid on a map of the seafloor-elevation. An additional simulated profile typical of the crest region outside of the canyon is also shown, revealing an apparent sampling bias due to the strategy of measuring weakly-stratified deep depressions along the trough of the canyon in search of the released tracer (Ledwell et al., 2000 ).

manifest themselves as spatial and temporal meanders of the mean up-canyon jet, which we show in the following section contribute significantly to the simulation's buoyancy budget and likely contribute to maintaining its strong stratification.

\subsection{Buoyancy budgets: mixing, mean flow, and eddies}

In Figure 3-7, we present volume-integrated buoyancy budgets (eq. 3.16) across our model hierarchy, time-averaged over days 5000 to 5200. All of the solutions exhibit substantial residual tendencies in the interior, but tendencies are an order of magnitude smaller than other terms in the budgets within a few hundred meters of the ridge flanks and within the canyons (Figure 3-7, black). The 1D and $1 \mathrm{D}_{\sigma=230}$ simulations are computationally inexpensive, so we also provide their fully-equilibrated solutions for context (Figure 3-7, b; dotted).

In the classical 1D solution, a weak up-slope transport in the BBL (Figure $3-7$ a, blue line) maintains a weak near-boundary stratification (Figure 3-8, solid black/grey), as discussed in Section 3.2.2. The evolution of the Smooth3D solution follows the 1D solution closely until about 800 days, at which point the laminar solution becomes unstable to submesoscale baroclinic modes (see linear 


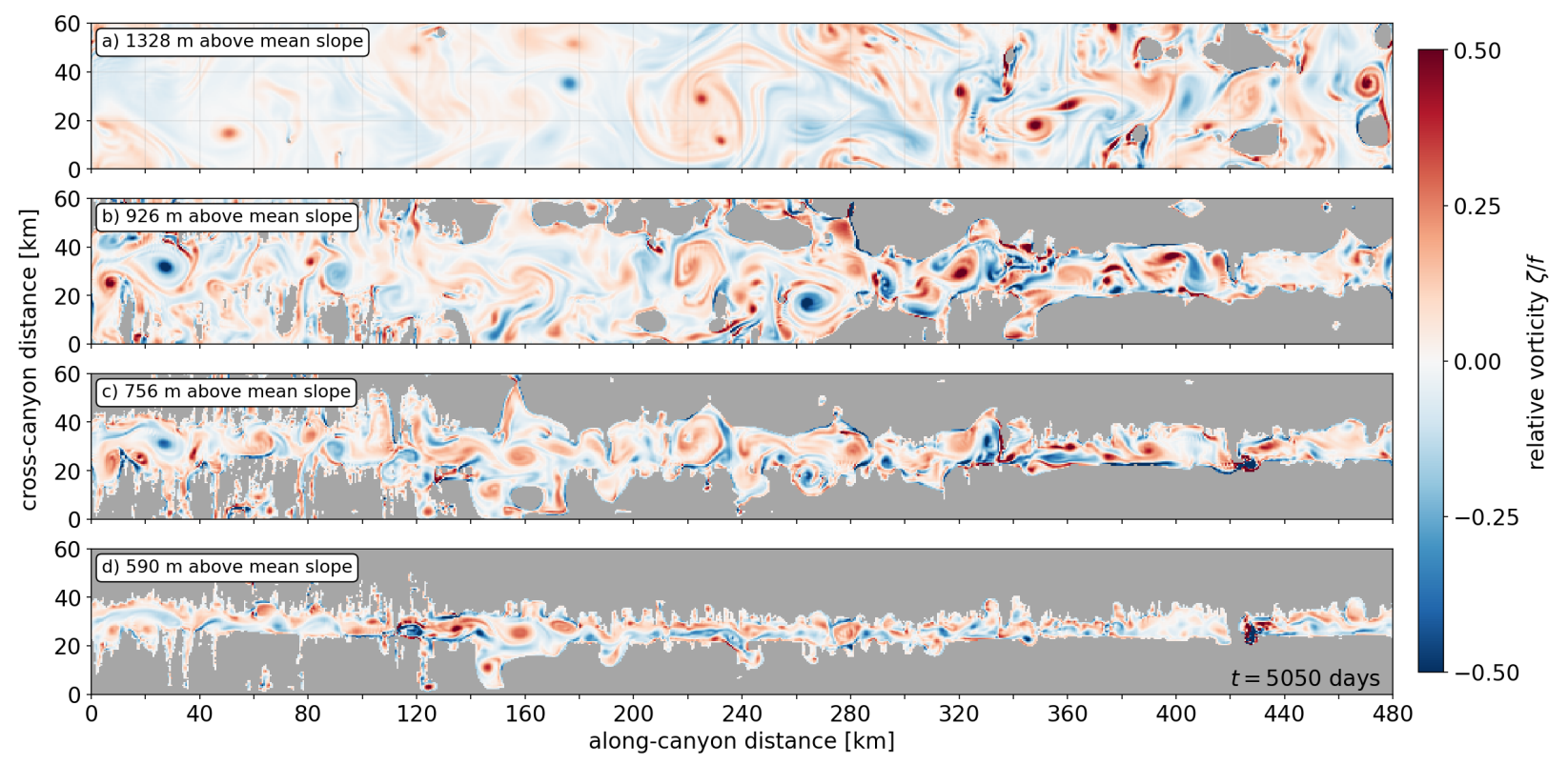

Figure 3-6: Instantaneous normalized relative vorticity $\zeta / f$, or local Rossby number, in and above the BBTRE Canyon at four different heights above the mean slope, at $t=5050$ days.

stability calculations in Wenegrat et al. 2018) which rapidly grow and equilibrate at finite amplitude (see Callies, 2018). At quasi-equilibrium, these transient eddies transport buoyancy from the BBL to the SML (Figure 3-7b, orange), effectively restratifying the BBL (Figure 3-8, blue) and thus strengthening the maximum turbulent buoyancy flux (Figure 3-7b, red). Relative to the 1D model, these additional eddy and turbulent buoyancy fluxes exactly cancel, such that the transient upslope transport and residual tendencies remain identical to those of the 1D model (Figure 3-7a,b; blue and black lines, respectively); it is unclear whether this unexpected result is just a transient coincidence or a fundamental property of the equilibrium solution. The crude eddy parameterization in $1 \mathrm{D}_{\sigma=230}$ qualitatively captures this restratifying effect ${ }^{6}$ (Figure 3-8 , compare dash-dotted and blue with solid grey) and enhances the maximum buoyancy flux into the BBL by a factor of 3 relative to the $1 \mathrm{D}$ model, both transiently and at equilibrium (Figure 3-7b,c; solid and dotted red lines, respectively). However, since the $1 \mathrm{D}_{\sigma=230}$ model does not permit explicit eddy fluxes, the parameterized effects of eddy fluxes are instead represented as an enhanced cross-slope transport due to less energy being partitioned to the along-slope thermal wind shear (Figure 3-7k; blue).

The volume-integrated buoyancy budget is more complicated to interpret in the presence of variable topography. In the Canyon solution, a substantial turbulent buoyancy flux convergence

\footnotetext{
${ }^{6}$ The available potential energy in our simulations has to build up from zero, in contrast to Callies (2018)'s solutions seeded with substantial available potential energy; thus, the parameterized restratification in $1 \mathrm{D}_{\sigma=230}$ is too strong and causes us to overestimate Smooth3D's transient restratifying effect.
} 

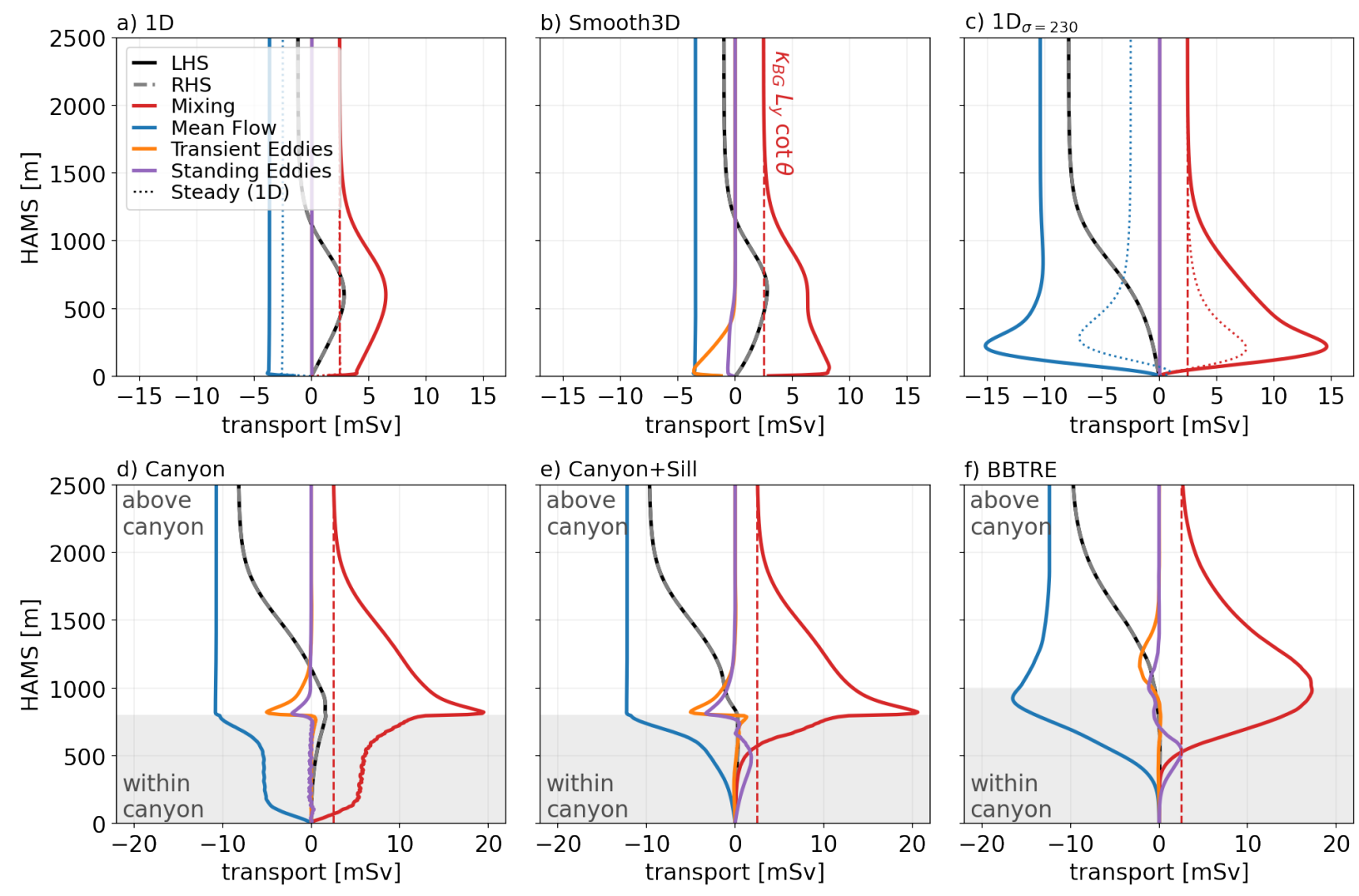

Figure 3-7: Generalized integral buoyancy budget in a hierarchy of increasingly complex simulations of mixing-driven flows up a mean slope of angle $\theta$ : (a) 1D, (b) Smooth3D, (c) $1 \mathrm{D}_{\sigma=230}$, (d) Canyon, (e) Canyon+Sill, (f) BBTRE. Solid lines show terms of the volume-integrated buoyancy budget (eq. 3.16) for a layer bounded by a given Height Above the Mean Slope (HAMS): residual tendencies are the result of a three-way balance between mean cross-slope advection, slope-normal turbulent mixing, and vertical eddy fluxes. We divide by the factor $N^{2} L_{x} \sin \theta$ to conveniently express the budget in terms of the quantity of interest, the up-slope volume transport $\Psi$ with units of mSv $\stackrel{\text { def }}{=}$ $10^{3} \mathrm{~m}^{3} / \mathrm{s}$. Dotted lines in (a,c) show 1D steady state solutions and the dashed red line shows the integral constraint (eq. 3.13); in panel a, the dotted and dashed red lines overlap. Grey shading shows the HAMS range spanned by the canyon, if present.

drives a vigorous up-slope mean flow within the bottom $200 \mathrm{~m}$ along the narrow trough of the canyon $5 \mathrm{mSv}$ (Figure 3-7 d, blue), which is already larger than the total BBL transport in the 1D model (Figure 3-7a, blue). The stratification in the trough of the Canyon is efficiently maintained near the large background value (Figures 3-9b; 3-8f, orange line). Thurnherr and Speer (2003) and Ruan and Callies (2020) hypothesize that this efficient restratification is due to the canyon sidewalls blocking the along-ridge thermal wind, such that the momentum is redirected into the cross-slope flow. The $1 \mathrm{D}_{f=0}$ model's excellent agreement with the stratification in the Canyon trough supports this hypothesis (Figure 3-8; orange and dotted lines). 

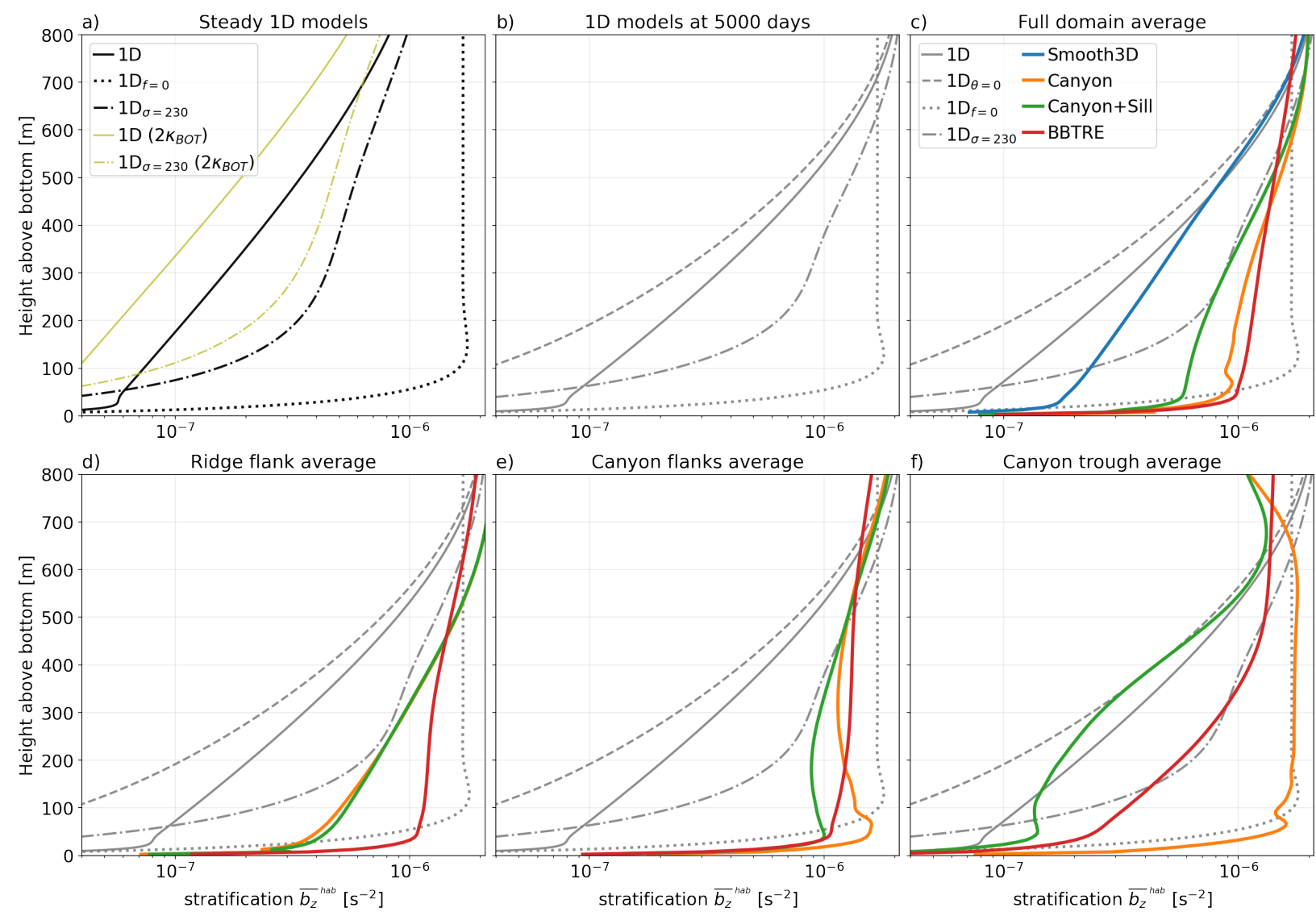

Figure 3-8: Height above bottom-averaged stratification profiles at steady state (a) and at $t=$ 5000 days (b-f), as a function of model complexity (lines) and domain sub-region (lower row). Panels $(\mathrm{a}, \mathrm{b})$ and grey lines in (d-f) show one-dimensional Dedalus solutions with: the same parameters as the BBTRE simulations (solid), with zero mean-slope $(\theta=0$; dashed), without rotation $(f=0$; dotted), and with a high turbulent Prandtl number $(\sigma=230$, crude proxy for restratification by baroclinic eddies; dash-dotted). Colored lines show a hiearchy of three-dimensional simulations with increasingly complex topographies (see Figure 3-2 -f).

The turbulent buoyancy flux also converges around a Height Above the Mean Slope (HAMS) of $z=800 \mathrm{~m}$, at the transition between the canyon flanks and the smooth ridge flank, driving a substantial additional upwelling of about $15 \mathrm{mSv}$ from $z=600 \mathrm{~m}$ to $800 \mathrm{~m}$ (Figure 3-7 $\mathrm{d}$ ). Upwelling along the smooth ridge flank of the Canyon simulation is about twice as large as that of the Smooth3D simulation, despite covering a smaller area, because some of the high stratification at the crest of the canyon flanks is exported out of the canyon and onto the BBL on the smooth ridge flank (Figure 3-9b,d) by an isopycnal eddy thickness flux and maintains a much larger stratification there (Figures 3-8c,d,e). In the Canyon simulation's quasi-equilibrium state, virtually all of the turbulent buoyancy flux divergence in the upper SML (far above the seafloor) is not yet equilibrated: the bottom-enhanced diffusion of buoyancy towards the boundary slowly cools the interior (Figure 3 
7d, red and black lines).
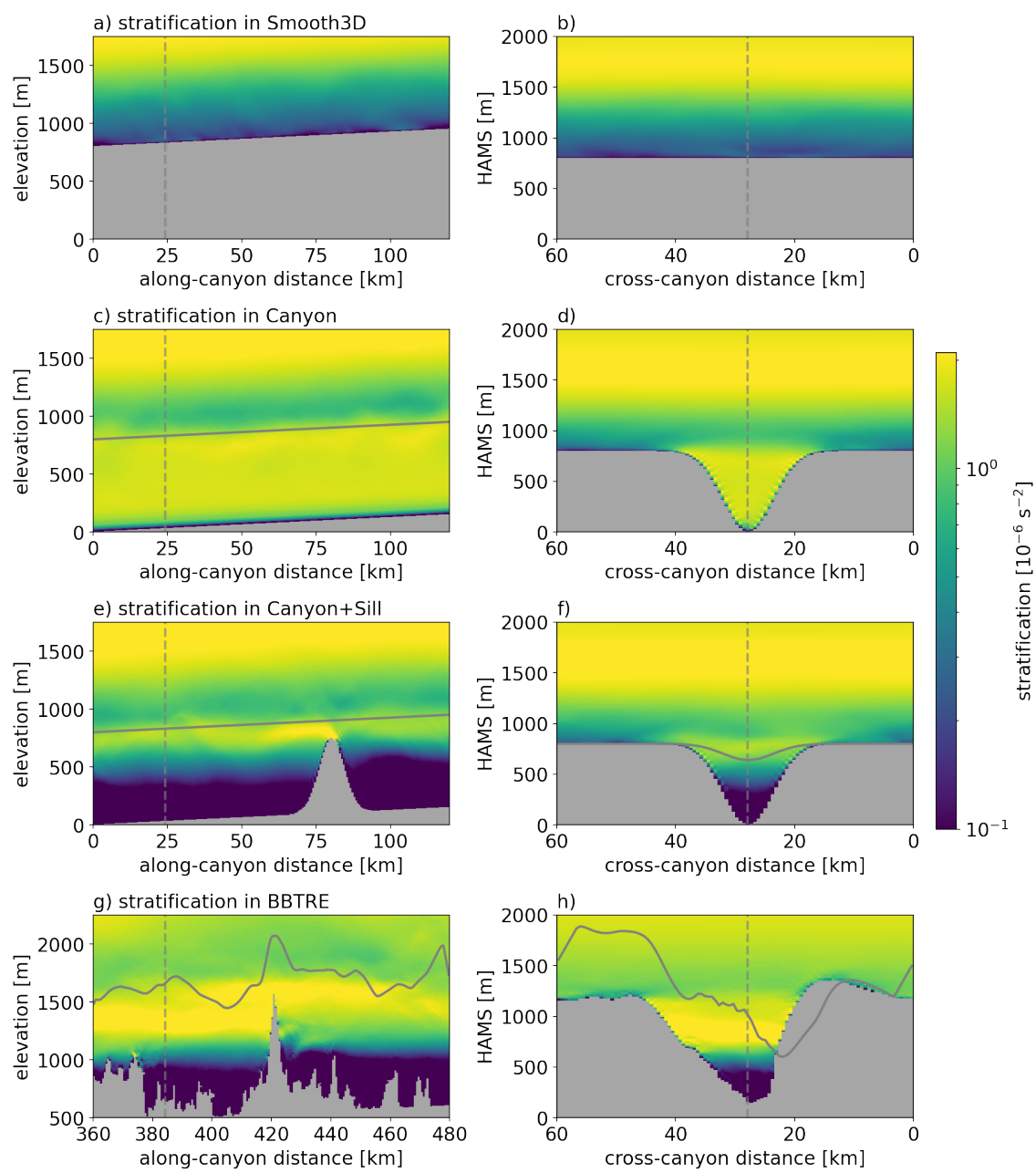

Figure 3-9: Cross-slope (left) and along-slope (right) sections of the stratification along the trough of a canyon in a hierarchy of numerical simulations (Smooth3D has no canyon, so the section is arbitrary). Solid grey lines in the left column show the approximate elevation of the ridge flanks surrounding the canyon while in the right column they show HAMS of the topographic sill (if present). Dashed grey lines show the locations of the respective sections.

In the Canyon + Sill simulation, the sill blocks up-slope flow within the trough of the canyon (Figure 3-7, e vs. d). This is expected, since the up-canyon flows of $\mathcal{O}(1 \mathrm{~cm} / \mathrm{s})$ only carry sufficient kinetic energy to lift a parcel across a stratification of $N \sim \mathcal{O}\left(10^{-4}-10^{-3} \mathrm{~s}^{-1}\right)$ by a height $\delta_{\mathrm{Fr}}=$ $U / N \sim 20-200 \mathrm{~m}$ (based on a topographic Froude number of $\mathrm{Fr} \stackrel{\text { def }}{\equiv} N \delta_{\mathrm{Fr}} / U \sim 2$ ), much smaller than the sill height of $h_{\text {sill }}=800 \mathrm{~m}$ and resulting in a blocked flow layer of thickness $h_{\text {sill }}-\delta_{\mathrm{Fr}}$ Baines, 1979. Winters and Armi, 2012), both up- and down-stream of the sill (recall also the periodic boundary conditions). No up-slope mean flow is available to restratify the trough of the Canyon, so it slowly homogenizes due to mixing (Figure 3-9 ); the evolution of the trough's stratification 
closely follows that of a diffusely-growing boundary layer over a flat bottom (compare with our $1 \mathrm{D}_{\theta=0}$ simulation; green and dashed grey lines in Figure $3-8 \mathrm{f}$ ), suggesting that, in the absence of additional restratification processes, the trough will eventually completely homogenize $\underbrace{7}$ (as in Dell 2013). In contrast, within a slope-normal displacement $\delta_{F r}$ of the sill, mean flows along the two flanks of the canyon are able to maintain a layer of strong stratification (Figures 3-7e, 3-9e,f, and $3-8 \mathrm{e})$; as in the Canyon simulation, the strong stratification in this thin layer spills over onto the smooth ridge flanks and maintains a larger stratification there than in the Smooth3D case (Figure $3-9$,, $\mathrm{b}$ and $3-8 \mathrm{e}, \mathrm{d})$.

The structure of the stratification in the BBTRE simulation is qualitatively similar to that of the Canyon+Sill simulation, although the rougher abyssal hill topography acts to thicken the layer of enhanced stratification near the DoMORE sill height and supports a large near-bottom stratification on the hilly ridge flanks surrounding the canyon (Figure $3-9 \mathrm{~g}, \mathrm{~h}, 3-8 \mathrm{~d}$ ). The slopenormal structure of the BBTRE canyon's buoyancy budgets (Figure 3-7f) is remarkably similar to that of the Canyon+Sill simulation, and can be broken down into several key elements:

1. cross-canyon sills block up-slope flow along the canyon trough, such that mixing slowly erodes its stratification;

2. along the crests of the canyon flanks $\left(\delta_{F r}\right.$ below the sill height and above), a significant upslope transport maintains a layer of strong stratification that separates the weakly-stratified canyon trough from the interior;

3. a combination of transient and standing submesoscale eddies maintain a strong near-bottom stratification along the ridge flanks, which supports a large turbulent buoyancy flux conver gence in BBLs;

4. bottom-enhanced mixing drives a large buoyancy flux divergence in the SML, which compen sates for most of the convergence in the canyon and the BBLs (by construction, see eq 3.13); however, due to its slow diffusive timescale, by day 5000 only a small fraction of this buoyancy flux divergence is balanced by down-slope transport.

\footnotetext{
${ }^{7}$ Tidal velocities, omitted for simplicity here, would imply a larger excursion height, a thinner blocked flow layer, and the potential for restratification processes to penetrate deeper into the canyon trough (as hypothesized by Clément et al., 2017).
} 


\subsection{Mixing-driven watermass transformations}

A more natural framework for understanding the drivers of diapycnal transport is watermass trans formation analysis (Walin, 1982, Marshall et al., 1999), which reframes the buoyancy budget in buoyancy space by integrating along buoyancy surfaces (or over buoyancy classes). Following Fer rari et al. (2016), the diapycnal transport $\mathcal{E}(b, t)$ across a buoyancy surface $\mathcal{A}(b, t)$ is given by

$$
\mathcal{E}(b, t) \stackrel{\text { def }}{=} \iint_{\mathcal{A}(b, t)} \mathbf{e} \cdot \mathbf{n} \mathrm{d} A=\partial_{b} \int_{\mathcal{V}(\tilde{b}<b)} \nabla \cdot(\kappa \nabla \tilde{b}) \mathrm{d} V,
$$

where $\mathbf{e} \stackrel{\text { def }}{\equiv}\left(\mathbf{u} \cdot \mathbf{n}-\frac{b_{t}}{|\nabla b|}\right) \mathbf{n}$ is the diapycnal velocity and $\mathcal{V}(\tilde{b}<b)$ is the volume enclosing any water denser than $b$. Since our simulations have not fully equilibrated in the SML, diapycnal transports include two components: flow across a buoyancy surface and the movement of the buoyancy surfaces themselves. In the present context, it is useful to decompose the diapycnal transport into a strictly upwelling BBL component $\mathcal{E}_{\mathrm{BBL}}$, where the integral is only evaluated over the strictly upwelling volume $\mathcal{V}(\tilde{b}<b ; \nabla \cdot(\kappa \nabla \tilde{b})>0)$, and a strictly downwelling SML component $\mathcal{E}_{S M L}$, similarly defined.

In practice, meaningful evaluation of this integral in the slope-native configuration requires stitching together $H /\left(L_{x} \tan \theta\right) \approx \mathcal{O}(5)$ periodic copies of the domain before adding in the back ground buoyancy field $B$, so that each isopycnal can be followed all the way from its incrop at the seafloor to the interior far-field where mixing is weak (Figure 3-10). Further, because our simulation is periodic in the cross-slope direction (and thus in mean buoyancy), the resulting watermass trans formations are periodic over a buoyancy interval $\Delta B=N^{2} L_{x} \tan \theta \approx 0.52{ }^{\circ} \mathrm{C}$. Temporal variability of watermass transformations is small relative to the other variations we focus on, so all results hereafter refer to their time-mean.

Net watermass transformations computed from $(3.18)$ are small $(\approx 2 \mathrm{mSv})$ and when averaged over the periodic buoyancy integral $\Delta B$ converge on the integral constraint $\kappa_{\mathrm{BG}} \cot \theta L_{y}$ (Figure 3-11a, black and dashed grey). However, this small net transformation is the residual of a large BBL upwelling $(60 \mathrm{mSv})$ and an almost-as-large SML downwelling (Figure 3-11a; solid red and blue squares, respectively). The ratio $\mathcal{E}_{\mathrm{BBL}} / \mathcal{E} \approx 30$, known as the amplification factor, quantifies the magnitude of upwelling in the BBL relative to the net overturning. Despite forcing watermass transformations by a uniform height-above-bottom diffusivity, $\mathcal{E}_{\mathrm{BBL}}(b)$ varies from 45 to $70 \mathrm{mSv}$ (Figure 3-11a; solid red and blue lines). 


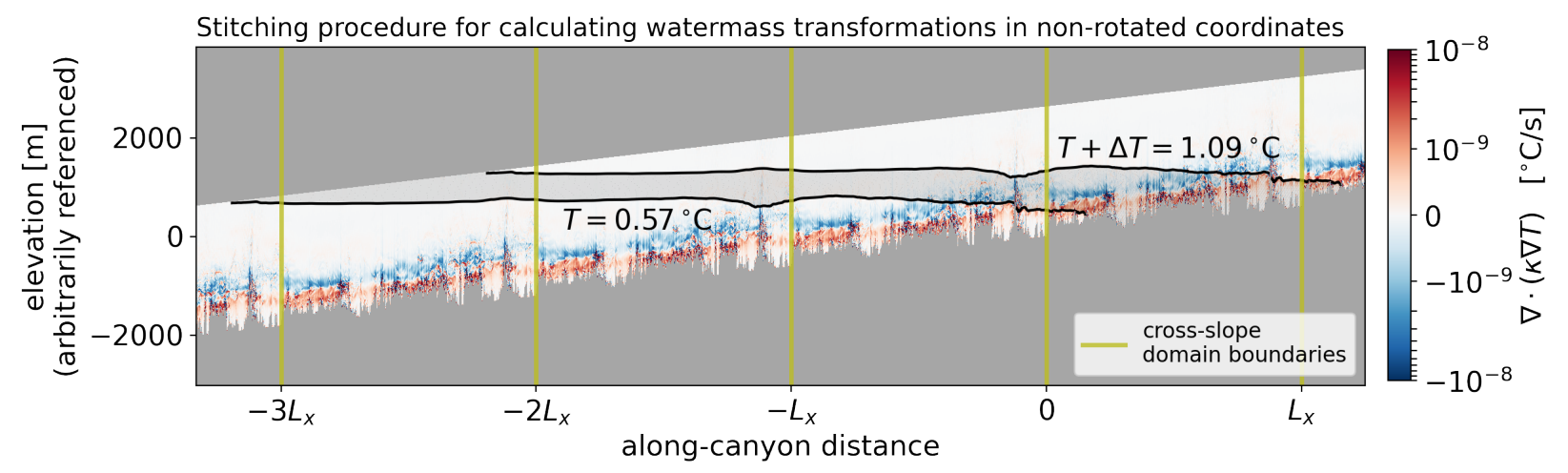

Figure 3-10: Turbulent buoyancy (temperature) flux convergence along the trough of the BBTRE canyon. Vertical olive lines show along-canyon boundaries of the simulation domain; the solution is doubly periodic in buoyancy perturbations, but discontinuous in the total buoyancy in the alongcanyon direction due to a constant background mean slope and stratification. For the small mean slopes considered here, computing watermass transformations thus requires reconstructing the full extent of buoyancy surfaces by stitching together multiple copies of the domain, each translated by a multiple of the domain extent $L_{x}$ and background temperature jump $\Delta T=\frac{N^{2}}{g \alpha} L_{x} \tan \theta \approx 0.52{ }^{\circ} \mathrm{C}$.

To understand these variations, we invoke the divergence theorem to rewrite the BBL watermass transformations in terms of fluxes across bounding surfaces,

$$
\mathcal{E}_{\mathrm{BBL}}(b)=\partial_{b} \iint_{\partial \mathcal{V}(\tilde{b}<b ; \nabla \cdot(\kappa \nabla b)>0)} \kappa \nabla \tilde{b} \cdot \mathbf{n} \mathrm{d} A
$$

Averaging over an arbitrary buoyancy interval $\left\{b-\frac{\Delta b}{2} ; b+\frac{\Delta b}{2}\right\}$, we find

$$
\overline{\mathcal{E}}_{\mathrm{BBL}}^{b \pm \Delta b}(b) \stackrel{\text { def }}{=} \frac{1}{\Delta b} \int_{b-\frac{\Delta b}{2}}^{b+\frac{\Delta b}{2}} \mathcal{E}_{\mathrm{BBL}} \mathrm{d} \tilde{b}=\frac{1}{\Delta b} \iint_{\partial \mathcal{V}\left(b-\frac{\Delta b}{2}<\tilde{b}<b+\frac{\Delta b}{2} ; \nabla \cdot(\kappa \nabla b)>0\right)} \kappa \nabla \tilde{b} \cdot \mathbf{n} \mathrm{d} A
$$

The right hand side of 3.20 can be broken into four different components (see schematic in Figure 3-1b): (A) the flux through the seafloor, which vanishes due to the no-flux boundary condition; (B) the flux across the transitional surface that separates the upwelling BBL from the downwelling SML, defined by $\nabla \cdot(\kappa \nabla b)=0$; (C) the flux across the $b-\frac{\Delta b}{2}$ surface intersected with the BBL; and (D) the flux across the $b+\frac{\Delta b}{2}$ surface intersected with the BBL. The contributions of (C) and (D) are much smaller than (B), both because the roughly horizontal surfaces of (B) cover a much larger area than the vertical surfaces of $(\mathrm{C})$ and $(\mathrm{D})$ (for the choice of $\Delta b \approx 0.05^{\circ} \mathrm{C}$ shown here) and because (C) and (D) are in opposite directions and largely cancel each other. A reasonable approximation of (B), the turbulent flux into the layer's BBL, is provided by approximating the transitional surface as a constant height-above-bottom surface, with hab ${ }_{\mathrm{BBL}}>\delta$ slightly larger than 
the typical BBL thickness; in practice, we find that hab $\mathrm{BBL}_{\mathrm{BB}}=40 \mathrm{~m}$ yields reasonable results. Since hab BBL $\ll h$ implies $\kappa($ hab $\mathrm{BBL}) \approx \kappa_{\mathrm{BOT}}$, we can pull $\kappa$ out of the integral in $(3.20)$, such that

$$
{\overline{\mathcal{E}_{\mathrm{BBL}}}}^{b \pm \Delta b} \approx \frac{1}{\Delta b} \kappa_{\mathrm{BOT}} \iint_{\mathcal{A}\left(\mathrm{hab}_{\mathrm{BBL}}\right)} \nabla \tilde{b} \cdot \mathbf{n} \mathrm{d} A \approx \frac{1}{\Delta b} \kappa_{\mathrm{BOT}}{\frac{\overline{\partial b}^{\text {hab }}}{\mathrm{BBL}}} A_{\text {incrop }}
$$

where we assume the integrated buoyancy gradient term is dominated by the slope-normal (or vertical) component and that the slopes of the $b \pm \frac{\Delta b}{2}$ buoyancy surfaces are sufficiently similar in the BBL that the $\mathcal{A}\left(\right.$ hab $\left._{\mathrm{BBL}}\right)$ surface at the top of the BBL is well approximated by the more readily available seafloor incrop area, $\iint_{\mathcal{A}_{\text {hab }_{\text {BBL }}}} 1 \mathrm{~d} A \approx A_{\text {incrop }}$ (as in de Lavergne et al. 2016c; see also schematic Figure $3-1 \mathrm{~b}$ ). This approximation reproduces the variations of $\overline{\mathcal{E}}_{\mathrm{BBL}}^{b \pm \Delta b}$ with buoyancy (Figure 3-11, red) and allows us to attribute them to two related effects: buoyancy variations in 1) the average near-bottom stratification (Figure 3-11b) and 2) the incrop area (Figure 3-11c). In particular, we highlight the $\left\{0.25^{\circ} \mathrm{C}, 0.35^{\circ} \mathrm{C}\right\}$ layer, which covers a large incrop area (including a large depression in the canyon trough) and exhibits weak mean stratification, and the $\left\{0.6^{\circ} \mathrm{C}, 0.7^{\circ} \mathrm{C}\right\}$ layer, which has a much smaller incrop area which mostly covers the stronglystratified canyon flanks (Figure 3-11d). The observed negative correlation between stratification and incrop area is expected, since large stratification (or small layer thickness $\frac{\mathrm{d} z}{\mathrm{~d} b}$ ) projects onto the local topogaphic slope $\nabla \hat{d}$ as a small area.

A plausible upper bound for BBL upwelling is given by

$$
{\overline{\mathcal{E}_{\mathrm{BBL}}}}^{b \pm \Delta b}<\kappa_{\mathrm{BOT}} \cot \theta L_{y}=85 \mathrm{mSv}
$$

where we assume the background stratification $N^{2}$ is a reasonable upper bound for the near boundary stratification and $A_{\text {incrop }} \approx \frac{L_{y} \Delta b}{N^{2} \sin \theta}$ is a geometrical estimate of the incrop area of a buoyancy layer with thickness $\Delta b$ given the background vertical stratification $N^{2}$ and slope angle $\theta$. Diapycnal upwelling in the BBL varies from $60 \%$ to $90 \%$ of this upper bound (Figure 3-11 ), a testa ment to the remarkable efficiency of the processes that maintain strong near-boundary stratification throughout most of the domain in the BBTRE simulation (Figure 3-11 p, d).

The detailed vertical (or HAB) structure of upwelling and downwelling watermass transforma tions are also of interest, since these are more directly comparable with measurements from vertical profilers and 1D BBL theory. Building upon Holmes and McDougall (2020), we define the height 

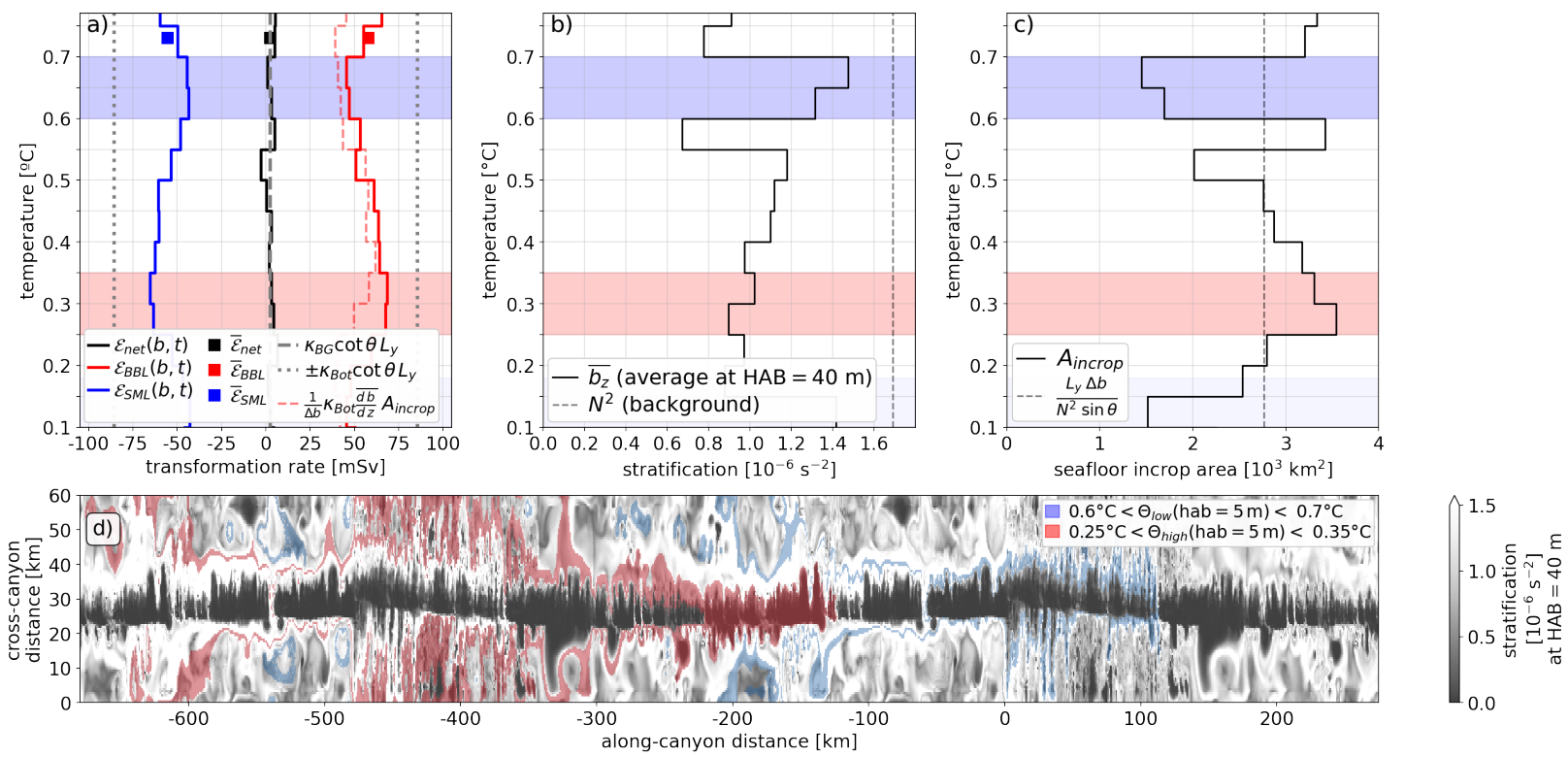

Figure 3-11: Drivers of watermass transformations in the BBTRE Canyon. (a) Positive, negative, and net watermass transformation rates as a function of temperature, averaged over many snapshots between days 5000 and 5500, in the BBTRE Canyon simulation. The dashed grey line represents the integral constraint on the net watermass transformation, which is set by the far-field flux of buoyancy into the domain. The dotted grey lines show an approximate upper bound on the positive and negative components of the watermass transformations, which is set by the maximum buoyancy flux $\kappa_{\text {Bot }} N^{2}$ expected in the domain. The red and blue shading in all panels delineate two temperature classes that exhibit particularly strong and weak positive transformation rates, respectively. We note that by construction the watermass transformations are periodic in temperature, such that the temperature class shaded in blue shows up again as a colder temperature class shaded in light blue (their average values across all times and temperatures are shown as squares). The dashed red line in (a) shows a simple scaling for the positive component of the watermass transformation rate, which approximates the total integrated buoyancy flux at the top of the BBL by a product of the bottom diffusivity $\kappa_{\text {Bot }}$, the average stratification $\frac{\overline{\partial b}}{\partial z}$ at the top of the thin BBL (b, d), and the area $A_{\text {incrop }}$ over which a temperature class incrops into the seafloor $(\mathrm{c}, \mathrm{d})$.

above-bottom watermass transformation structure as:

$$
\frac{\partial \mathcal{E}}{\partial \text { hab }} \stackrel{\text { def }}{=} \frac{\partial}{\partial \text { hab }}\left[\frac{\partial}{\partial b} \int_{\mathcal{V}\left(\tilde{b}<b ; \text { hab }^{\prime}<\text { hab }\right)} \nabla \cdot(\kappa \nabla \tilde{b}) \bar{d} V\right] .
$$

Consistent with the HAB-averaged stratification profiles in Figure 3-8, vigorous upwelling is on average confined to the $40 \mathrm{~m}$-thick BBL, with compensating weak but broad downwelling in the SML above; the notable exception is the canyon trough, whose maximum buoyancy flux is achieved $250 \mathrm{~m}$ above the bottom and which exhibits much weaker upwelling per unit area (Figure 3-12a). 


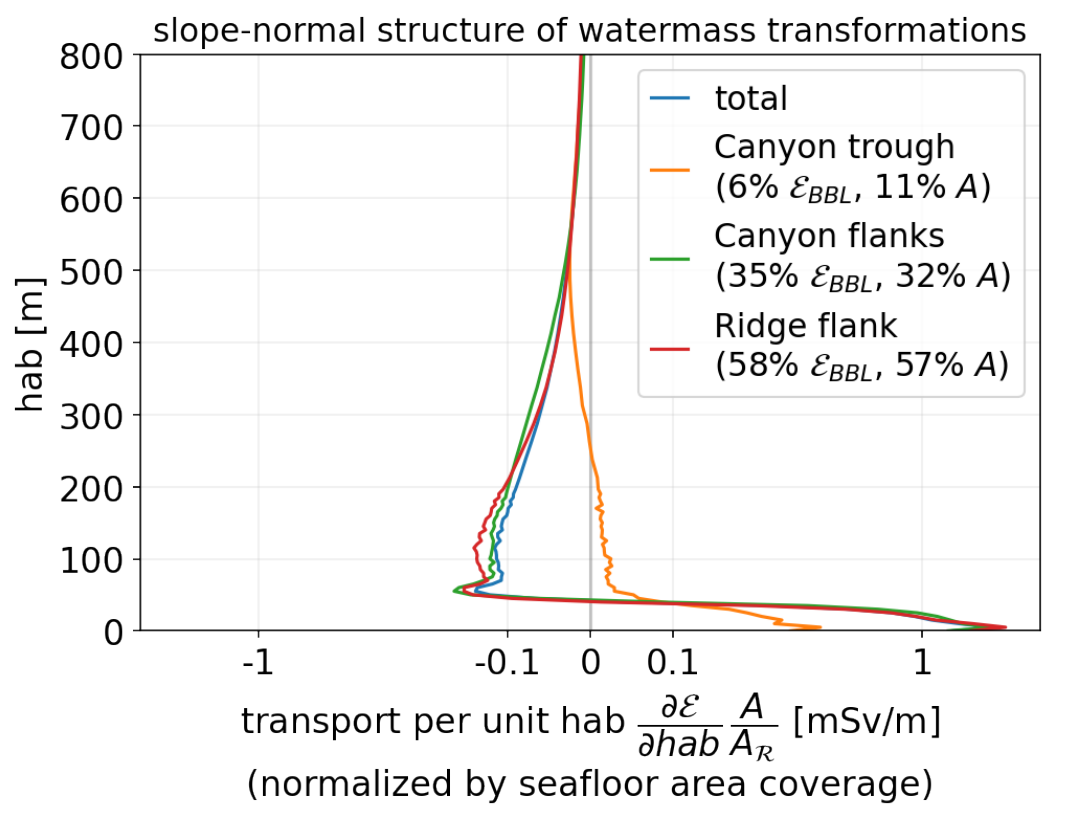

Figure 3-12: Height-above-bottom structure of watermass transformations (eq. 3.23) in three dynamically-distinct regions: the canyon trough, the canyon's sloping flanks, and the ridge flanks surrounding the canyon. To directly compare watermass transformations from differently-sized re gions, we normalize by their area $A_{\mathcal{R}}$ and scale them up to the domain area $A=L_{x} L_{y}$. Percentages reported in the legend quantify the relative contributions of BBL upwelling and surface area of each region, highlighting the inefficiency of upwelling in the weakly-stratified canyon trough.

\subsection{Conclusions and Discussion}

By generalizing the methods of classical 1D sloping Bottom Boundary Layer (BBL) theory, we con struct simulations of 3D mixing-driven flows above sloping rough topography that permit non-trivial equilibrium states: the homogenizing tendency due to mixing is balanced by cross-slope advection across a background stratification, effectively providing an infinite reservoir of dense bottom waters. These slope-native solutions are more interpretable than previous model 4 , which either require aphysical sponge layers at distant horizontal boundaries (Dell, 2013) or can only be analyzed tran siently due to their eventual homogenization by mixing (Ruan and Callies, 2020). In particular, the slope-native model converges exactly upon the classical 1D model in the limit of smoothly sloping topography (and suppressed variations along the plane of the slope), allowing a robust hierarchy of models approach. While the local nature of the sloping BBL framework is conceptually convenient,

\footnotetext{
${ }^{8} \mathrm{~A}$ second advantage is that the large-scale slope is implicitly imposed and does not need to be explicitly resolved, permitting much high-resolution simulations without sacrificing the approximate effects of a large-scale mean slope. In the atmosphere, a somewhat similar approach applies the "Weak Temperature Gradient" approximation of the large-scale tropical circulation (Sobel et al., 2001) to cloud-resolving simulations of radiative-convective equilibria (e.g. Abbott and Cronin 2021).
} 
ignored non-local factors may be equally important. For example, externally-imposed along-slope flows (e.g. boundary currents) spin-up bottom Ekman layers which are arrested by buoyancy forces (MacCready and Rhines, 1991; Garabato et al., 2019). Additionally, imposing a external cross-slope pressure gradient that ensures the net up-slope transport vanishes (as required by continuity in a finite-depth ocean) fundamentally alters the transient spin-up problem, motivating a transportconstrained reformulation of sloping 1D BBL theory (Peterson and Callies, in prep).

The results of our quasi-realistic simulation of the Brazil Basin Tracer Release Experiment (BBTRE) unifies two dominant boundary mixing paradigms: yes, bottom-enhanced mixing drives up-slope flow in the BBL; but, this flow is much stronger than predicted by 1D theory (Garrett, 1979 , 1990) due to the net restratification by transient baroclinic eddies and topographic steering/blocking Armi, 1978; Thurnherr and Speer, 2003, Callies, 2018; Ruan and Callies, 2020). These restratifying effects can to a large extent be attributed to three distinct physical processes:

1. slumping of isopycnals by finite-amplitude submesoscale baroclinic instabilities (Wenegrat et al., 2018; Callies, 2018),

2. the blocking of rotational effects within narrow fracture zone canyons (Thurnherr and Speer, 2003; Dell, 2013; Ruan and Callies, 2020, and

3. the effect of sills in blocking up-canyon mean flows and homogenizing depressions well below the sill height (Baines, 1979; Winters and Armi, 2012, Dell, 2013).

An idealized Canyon + Sill simulation, which minimally permits all three of these processes, qualita tively reproduces the flow structures of the realistic-topography BBTRE simulation; quantitatively, however, the addition of rough abyssal hills in the BBTRE simulation results in a near-bottom stratification that is twice as large as in Canyon+Sill. Attributing this additional restratification to a specific process is challenging because abyssal hill roughness affects both the mean mixing-driven boundary layer flow (Dell, 2013) and the properties (e.g. growth rates and length scales) of the mean flow's instabilities (e.g. Szoeke, 1983; Wenegrat et al., 2018; Radko, 2020); we leave this to future work.

Our quasi-realistic simulations provide the first BBL-resolving simulations of the mixing-driven abyssal overturning in the Brazil Basin, complementing Huang and Jin (2002) and Ogden and Fer rari (in prep)'s coarser-resolution basin-scale simulations. Despite the extreme idealization of the numerical set-up (spin-up from rest, only forced by mixing across background stratification), we qualitatively reproduce key features of the observations: broad up-slope flow and near-boundary 
stratification of $b_{z} \approx \mathcal{O}\left(10^{-7} \mathrm{~s}^{-2}\right)$ along the canyon trough (Toole, 2007; Polzin et al., 1997); hydraulically-accelerated flow over blocking sills (Clément et al., 2017); and the mean diapycnal downwelling and spreading of a tracer released in the SML (Ledwell et al. 2000; see also Chapter 4 for an updated discussion of these estimates). Despite this qualitative agreement, the simulated diapycnal transports are too weak-and the stratification too strong-by roughly a factor of 2 . There are several possible reasons for these remaining discrepancies. First, we speculate that the specu late that the observed dissipation rates may be biased low due to sampling biases (e.g., see Watson et al. 1988; Voet et al., 2015, Cael and Mashayek, 2021); biases in the mixing parameterization (Ijichi et al. 2020), such as the conventional assumption that the flux coefficient is $\Gamma=0.2$ (Osborn, 1980). Indeed, based on observations and basin-scale simulations of tracer spreading, both Ledwell (in prep) and Ogden and Ferrari (in prep), respectively, similarly conclude that tracer observations are more consistent with a diffusivities about 2 times larger than those inferred from microstructure 9 Ignored external factors such as along-slope currents (MacCready and Rhines, 1991; Benthuysen and Thomas, 2013) or cross-slope pressure gradients could also explain the remaining discrepancies (Peterson and Callies, in prep.).

The relative contributions of canyon troughs, canyon flanks, and the hilly ridge flanks surround ing the canyons (or "crests") to basin-wide abyssal watermass transformations are the subject of a long-standing debate (e.g. St. Laurent et al., 2001; Thurnherr and Speer, 2003; Thurnherr et al., 2005, Polzin, 2009: Dell, 2013, Callies, 2018; Thurnherr et al., 2020; Ruan and Callies, 2020). Based on observations of large dissipation rates and strong up-canyon flows at the DoMORE sill and similar hydrographic features in other fracture zone canyons, Thurnherr et al. (2020) argues that virtually all lightening of bottom waters occurs in fracture zones. Ruan and Callies (2020)'s idealized simula tions suggest that watermass transformations may be disproportionately large within canyons due to strong restratification by up-canyon jets, although they acknowledge that this is a direct result of unrealistically large stratification in the canyons. Our more realistic simulations show that sills block restratification of the canyon trough, resulting in much weaker watermass transformations. Combined with the more expansive and strongly-stratified canyon flanks, however, the full canyon system (trough and flanks) contributes just as much watermass transformation, per unit area, as the surrounding ridge flank (a bit less, in absolute terms, since the canyon covers only $40 \%$ of the total domain area). These results only hold if mixing is uniformly bottom-enhanced; if mixing is

\footnotetext{
${ }^{9}$ Given the uncertainties of the microstructure methods, agreement within a factor of 2 is generally considered to be good (e.g. Gregg et al. 2018).
} 
stronger in the canyons, as suggested by some available observations (e.g. St. Laurent et al. 2001; Clément et al. 2017; Ijichi et al. 2020), then their watermass transformations would be likely be proportionally stronger (e.g. following eq. 3.21). This characterization of strong mixing in canyons is not uniformly accepted, however, with others claiming the exact opposite pattern (Polzin, 2009).

Because of the geological origins of the characteristic topographic features in the BBTRE (large scale slope, canyon, and hills), the dynamics described can be thought to apply to the global midocean ridge system (with the steepness of slopes and hills modulated by the age of the rift valley). Extrapolating the $60 \mathrm{mSv}$ of diapycnal upwelling implied by watermass transformations (Figure 3-11) to the length of the Mid-Atlantic Ridge in the Brazil Basin (30 to 40 times the domain width $L_{y}=60 \mathrm{~km}$ ), the upwelling transport of $\mathcal{E}_{B B L}=1.8-2.4 \mathrm{~Sv}$ is comparable to $4 \mathrm{~Sv}$ inflow of Antarctic Bottom Water into the Brazil Basin (Hogg et al., 1982, Morris et al., 2001, Polzin, 2009. Clément et al. 2017); extrapolating to a global mid-ocean ridge system of length $40 \times 10^{3} \mathrm{~km}-$ $100 \times 10^{3} \mathrm{~km}$ (including both flanks of the ridge) leads to a global BBL upwelling of $40 \mathrm{~Sv}-100 \mathrm{~Sv}$ due to upwelling along mid-ocean ridges (Thurnherr et al., 2005, Callies, 2018, respectively), much larger than estimates of the net diabatic overturning and consistent with global diagnostic estimates of BBL upwelling (Ferrari et al., 2016, McDougall and Ferrari, 2017).

Global extrapolations of localized estimates of BBL upwelling, such as the above, have been used to attribute the net abyssal overturning to specific processes (Thurnherr et al., 2020; Spingys et al., 2021; Callies, 2018); these arguments, however, ignore the possibility that this localized upwelling is largely offset by related downwelling elsewhere along the same buoyancy surface, which would render these quantitative comparisons meaningless. For example, Thurnherr et al. (2020) argue that the observed turbulent buoyancy flux convergence in the BBTRE canyon, extrapolated to all of the fracture canyons in the Brazil Basin, is sufficient to transform "the total inflow of AABW". Above the canyon, however, their own observations imply an opposing buoyancy flux divergence of comparable magnitude: following a single buoyancy surface in their Figure 3, we infer from the buoyancy flux profiles in their Figure 7 that diapycnal upwelling within the Canyon at the DoMORE site will be significantly offset by diapycnal downwelling above the canyon at the BBTRE site. In a similar vein, Callies (2018) argues that submesoscale eddies maintain a sufficiently large stratification at the top of the BBL to support a BBL upwelling comparable to the observed $\mathcal{O}(30 \mathrm{~Sv})$ of abyssal overturning. This argument neglects, however, that the 1D integral constraint implies SML downwelling of a similar magnitude, such that the net transport reduces to the negligiblysmall integral constraint $\psi_{\infty}=\kappa_{\mathrm{BOT}} \cot \theta$ (eq. 3.13. At a global scale, diagnostics estimates of 
watermass transformations exhibit typical amplification factors $\mathcal{E}_{\mathrm{BBL}} / \mathcal{E}$ of 2 to 5 Ferrari et al. 2016; McDougall and Ferrari, 2017; Cimoli et al., 2019), suggesting that significant compensation is the norm 10 . Since the quantity of interest- the net diabatic overturning- is the small residual of two large compensating components, errors in either component risk dramatically biasing estimates. The two examples above are perhaps extreme, since we expect the SML downwelling to eventually vanish at the base of the mid-ocean ridge, where the sloping flanks give way to the abyssal plains Callies and Ferrari 2018b; Ruan and Callies 2020; Chapter 2) but they nevertheless illustrate the risk of misinterpretation.

Relatedly, the gravest caveat of this work is the assumption of a constant background stratifica tion. We know from Salmun et al. (1991)'s asymptotic analysis that small perturbations away from a constant background stratification drive a "tertiary circulation" that exchanges fluid between the boundary and the interior. In the context of the abyssal ocean, vertical variations in the basin-scale interior stratification are relatively large (Spingys et al., 2021); they enter as leading order terms in watermass transformations and drive substantial exchange between the mixing layers and the interior (Holmes et al. 2018). In the idealized basin-scale simulations of Chapter 1, we found that the decrease of stratification with buoyancy (or depth) offsets much of the sinking in the SML for realistic abyssal values, such that the net overturning scales roughly with upwelling in the BBL (with amplification factors of $\mathcal{E} / \mathcal{E}_{\mathrm{BBL}}=1.5$ to 2 ), in contrast to the negligibly small integral con straint implied by $1 \mathrm{D}$ theory ( $\mathcal{E}$ is so small that amplification factors become meaningless). The dynamical coupling between mixing layers and the interior remains poorly understood; improved understanding of this process seems to be a pre-requisite of developing improved models of the abyssal overturning circulation. The asymptotic model of Salmun et al. (1991), though an impres sive exercise, is sufficiently complicated and opaque to interpretation that it is not clear whether it yields any additional insights relative to a conceptually simpler configuration in a general circulation model (such as the PGCM used in Chapter 2, or the MITgcm used here); for completeness, I include in Appendix A my preliminary attempts at deriving a minimal asymptotic model (using the simpler Rayleigh drag parameterization of Chapter 2 that might clarify the dynamical coupling between abyssal mixing layers and the interior.

\footnotetext{
${ }^{10}$ Full range goes towards $\pm \infty$, since the amplification factor blows up as the net overturning goes to \pm 0 (e.g. Figure 9 of Ferrari et al. 2016).
} 


\section{Chapter 4}

\section{Diapycnal motion, diffusion, and stretching of tracers above rough topography}

\subsection{Introduction}

The lower limb of the ocean's meridional overturning circulation traces the diabatic life cycle of abyssal bottom waters (Talley, 2013a), which store vast quantities of climatically-active tracers like heat and carbon. Bottom waters are formed at the surface of the Southern Ocean by atmospheric cooling and brine rejection and are consumed in the deep ocean by buoyancy-flux convergence due to small-scale mixing and geothermal heating (Abernathey et al., 2016; de Lavergne et al., 2016c). Since mixing processes are too small to be resolved by large-scale ocean models, the rate at which tracers are mixed across density surfaces- the diapycnal diffusivity- enters as a key free parameter in ocean and climate models (Bryan and Lewis, 1979, Simmons et al., 2004, Lavergne et al., 2020). While early models of the abyssal circulation assume this mixing to be spatially uniform Munk, 1966, Stommel and Arons, 1959a), subsequent in-situ observations reveal a complex geography of mixing processes (e.g. Polzin et al., 1997; Waterhouse et al., 2014). A robust pattern that emerges from these in-situ mixing observations is the bottom-enhancement of mixing over rough topography, consistent with theoretical arguments that this mixing is predominantly caused by breaking internal waves radiating from flow over topography (Polzin, 2009, Nikurashin and Ferrari, 2009, Nikurashin and Legg, 2011; MacKinnon et al., 2017).

The observed bottom-enhancement of deep mixing demands a revision to classic abyssal circu lation theory: bottom-enhanced mixing above rough topography drives diapycnal downwelling in the stratified interior- or Stratified Mixing Layer (SML)- and an even larger diapycnal upwelling 
in the Bottom Boundary Layer (BBL) due to convergence of buoyancy at the seafloor Huang and Jin, 2002, Ferrari, 2014; Ferrari et al., 2016; de Lavergne et al., 2016c; McDougall and Ferrari, 2017, Callies and Ferrari, 2018b). Geothermal heat flux into the BBL acts to amplify upwelling, but is thought to be secondary to mixing globally (de Lavergne et al., 2016b) and is negligible in the Brazil Basin subregion considered here (Thurnherr et al., 2020). The global diabatic overturning circulation is the small residual of substantial downwelling in the SML and even larger upwelling in the BBL (Ferrari et al. 2016 and Chapter 2). While the existence of these upwelling/downwelling flows is virtually guaranteed by the combination of a bottom-enhanced turbulent buoyancy flux and an insulating boundary condition along a sloping seafloor, their structure, magnitudes, and underlying dynamics remain poorly understood (Callies 2018 and Chapter 2). Since diapycnal (or vertical) velocities and fluxes are challenging to measure directly due to the ocean's small aspect ratio, indirect methods must be used to infer the flow, such as volume or buoyancy budgets. Watermass transformation analysis is a commonly-used framework which combines volume and buoyancy budgets to express diapycnal transport across a buoyancy surface in terms of the average turbulent buoyancy flux along the surface, which can be inferred from indirect observations (Walin, 1982 , Marshall et al., 1999; de Lavergne et al., 2016c; Ferrari et al., 2016).

There are several observational methods for estimating in-situ turbulent buoyancy fluxes and their corresponding diffusivities: 1) velocity variance microstructure based on an approximate tur bulent kinetic energy budget (Osborn, 1980), 2) temperature variance microstructure based on an approximate temperature variance budget (Osborn and Cox, 1972), 3) shear/strain variance finestructure based on idealized spectral models of internal wave dynamics (e.g. Garrett and Munk, 1972, 1975, Henyey et al., 1986; Polzin et al., 1995, Gregg et al., 2003), and 4) scaling analysis based on the Thorpe scale of density overturns (Dillon, 1982), listed roughly in order of decreasing accuracy and generality (Gregg et al. 2018). All of these methods are indirect and require some degree of approximation to convert the measured quantity into a diffusivity. Furthermore, they provide only localized snapshots of spatially and temporally intermittent mixing events, and thus may provide biased estimates of the mean diffusivity.

Tracer Release Experiments (TREs; Watson et al. 1988) are considered by many (e.g. Gregg et al., 2018) to provide the gold standard of mixing rate estimates. In TREs, an assumed in ert chemical tracer (or fluorescent dye) is deliberately injected into the ocean and its distribution is surveyed over time. The evolution of the tracer is then typically inverted (using approximate advection-diffusion models) to yield estimates of the diffusivity and velocity, which can be com 
pared to other in-situ turbulence measurements. Localized TREs are experimental analogues of the Green's function and thus anticipate a strong diapycnal mixing signal by construction, especially in contrast to thermodynamic or biogeochemical tracers with more complicated dynamics and mix ing histories (e.g. Hogg et al., 1982; Lumpkin and Speer, 2007; Trossman et al., 2020). However, the results of the inversion process still depend upon the validity of the simple advection-diffusion forward models.

Watson et al. (1988) pitch TREs in the ocean as an "unambiguous measure of the diapycnal mixing rate" since tracers average over the spatial and temporal variability that challenges the inter pretation of in-situ mixing estimates; for example, they hypothesize that the sparse in-situ sampling of log-normally distributed (or worse, log-skew-normal) mixing events risks under-estimating mean mixing rates (Cael and Mashayek, 2021). Superficially, observations from two deep-ocean TREs seem to corroborate this hypothesis: tracer-based estimates of mixing rates are ubiquitously 1.510 times larger than co-located in-situ microstructure measurements (Ledwell et al., 2000; Watson et al. 2013, Mashayek et al., 2017a). While there are ad-hoc and site-specific explanations for each of these discrepancies, there is no consensus on how to compare tracer-based and microstructurebased estimates (Gregg et al., 2018), nor what to make of the fact that tracer-based estimates seem to always exceed microstructure-based estimates.

Recent advances in the numerical modelling and theory of TREs provide some guidance for in terpreting tracer-based estimates of mixing and comparing them to microstructure-based estimates. For example, Mashayek et al. (2017a) use a realistic regional simulation to argue that the mixing inferred from the DIMES TRE (Watson et al., 2013) was an order-of-magnitude larger than the in-situ diffusivity estimated from microstructure because the average diapycnal spreading of the tracer was dominated by a small fraction the tracer distribution that resided in regions of strong mixing near rough topography. Ruan and Ferrari (2021) derive exact evolution equations for the first and second tracer-weighted buoyancy moments (see Section 4.2.1), which confirm Mashayek et al. (2017a)'s speculation that the tracer's diapycnal variance grows with the tracer-weighted insitu diffusivity. However, Ruan and Ferrari (2021) also identify a second "diapycnal stretching" term through which the bottom-enhancement of the buoyancy velocity in the SML further acceler ates the diapycnal spreading of the tracer. Holmes et al. (2019) use a similar approach but include the effects of a sloping bottom boundary, and find that the diapycnal stretching in the SML is compensated for by a diapycnal contraction effect due to upwelling of relatively dense tracer in the BBL. However, since both of these analyses use extremely idealized models, it remains unclear to 
what extent diapycnal stretching affects tracers in realistic conditions.

In this paper, we present the first application of the buoyancy moments method in a quasirealistic TRE simulation. We use tracer-weighted buoyancy moments diagnosed from the simulation to explain the diapycnal downwelling and spreading observed in the Brazil Basin TRE (BBTRE; Ledwell et al. 2000) in the context of an emerging paradigm of bottom mixing layer control of the abyssal circulation (Ferrari et al., 2016, McDougall and Ferrari, 2017). We also provide guidance for the interpretation of past (e.g. Ledwell and Hickey, 1995; Ledwell et al., 2004, 2016, Visbeck et al., 2020) and future TREs near topography.

\subsection{Theory}

We briefly review a recently proposed framework for comparing tracer-based and microstructurebased mixing estimates, based on exact evolution equations for tracer moments in buoyancy space (Ruan and Ferrari, 2021). The derivation begins with the conservation equations for tracer concen tration $c$ and buoyancy $b$,

$$
\begin{aligned}
& \frac{\partial c}{\partial t}+\mathbf{u} \cdot \nabla c=\nabla \cdot(\kappa \nabla c) \\
& \frac{\partial b}{\partial t}+\mathbf{u} \cdot \nabla b=\nabla \cdot(\kappa \nabla b),
\end{aligned}
$$

where $\mathbf{u}$ is the velocity vector, $\nabla=\left(\frac{\partial}{\partial x}, \frac{\partial}{\partial y}, \frac{\partial}{\partial z}\right)$ is the gradient operator, and $\kappa$ is an isotropic turbulent diffusivity. Buoyancy, tracer concentrations, and velocity have been filtered on spatial and temporal scales larger than those associated with small-scale turbulence, and the filtered scalar fluxes are parameterized as an enhanced diffusive flux $\mathbf{F}_{\phi}=-\kappa \nabla \phi$, where the turbulent diffusivity is much larger than the molecular diffusivity. For simplicity of exposition, we here approximate density as a linear function of temperature; thus, density $\rho$, buoyancy $b$, and temperature $T$ are all proportional and will be used interchangeably throughout: $b \stackrel{\text { def }}{=}-g \frac{\rho}{\rho_{0}} \approx g \alpha T$, where $\rho_{0}$ is a reference density and $\alpha$ is the thermal contraction coefficient.

\subsubsection{Exact tracer-weighted buoyancy-moment models}

In his classic paper, Taylor (1922) demonstrates that the growth rate of half the second moment of a 1D tracer distribution in physical space is exactly equal to its diffusivity. Ruan and Ferrari (2021) generalize this theory to the case of variable diffusivity in a stably stratified fluid by considering 
moments in buoyancy space. By cross-multiplying the passive tracer 4.1 and buoyancy 4.2 equations and integrating over a volume $\mathcal{V}$ containing the tracer (or bounded by insulating and impermeable boundaries), they derive straightforward and exact evolution equations for the first and second tracer-weighted buoyancy moments,

$$
\begin{aligned}
& w_{\text {tracer }} \stackrel{\text { def }}{=} \frac{\partial_{t} \bar{T}}{\overline{|\nabla T|}}=\frac{2 \bar{\omega}}{\overline{|\nabla T|}} \quad \text { and } \\
& \underbrace{\frac{1}{2} \frac{\partial_{t} \overline{(T-\bar{T})^{2}}}{\overline{|\nabla T|^{2}}}}_{\begin{array}{c}
\kappa_{\text {tracer }} \\
\text { (spreading) }
\end{array}}=\underbrace{\frac{\overline{\left.|\nabla| \nabla T\right|^{2}}}{\overline{|\nabla T|^{2}}}}_{\begin{array}{c}
\kappa_{\text {Taylor }} \\
\text { (diffusion) }
\end{array}}+\underbrace{2 \frac{\overline{\omega^{\prime} T^{\prime}}}{\overline{|\nabla T|^{2}}}}_{\begin{array}{c}
\kappa_{\omega} \\
\text { (stretching) }
\end{array}}
\end{aligned}
$$

respectively, where overlines denote the tracer-weighted average, $-\stackrel{\text { def }}{=} \frac{\int_{\mathcal{V}} \cdot c \mathrm{~d} V}{\int_{\mathcal{V}} c \mathrm{~d} V} ;$ primes denote variations from the tracer average,,$\stackrel{\text { def }}{\equiv}---;$ and $\omega \stackrel{\text { def }}{\equiv} \nabla \cdot(\kappa \nabla T)$ is the in-situ buoyancy velocity, which is the magnitude of the diapycnal velocity in buoyancy space (Marshall et al., 1999). We have taken an additional step of converting to physical velocity and diffusivity units by normalizing by the appropriate tracer-weighted powers of the buoyancy gradient.
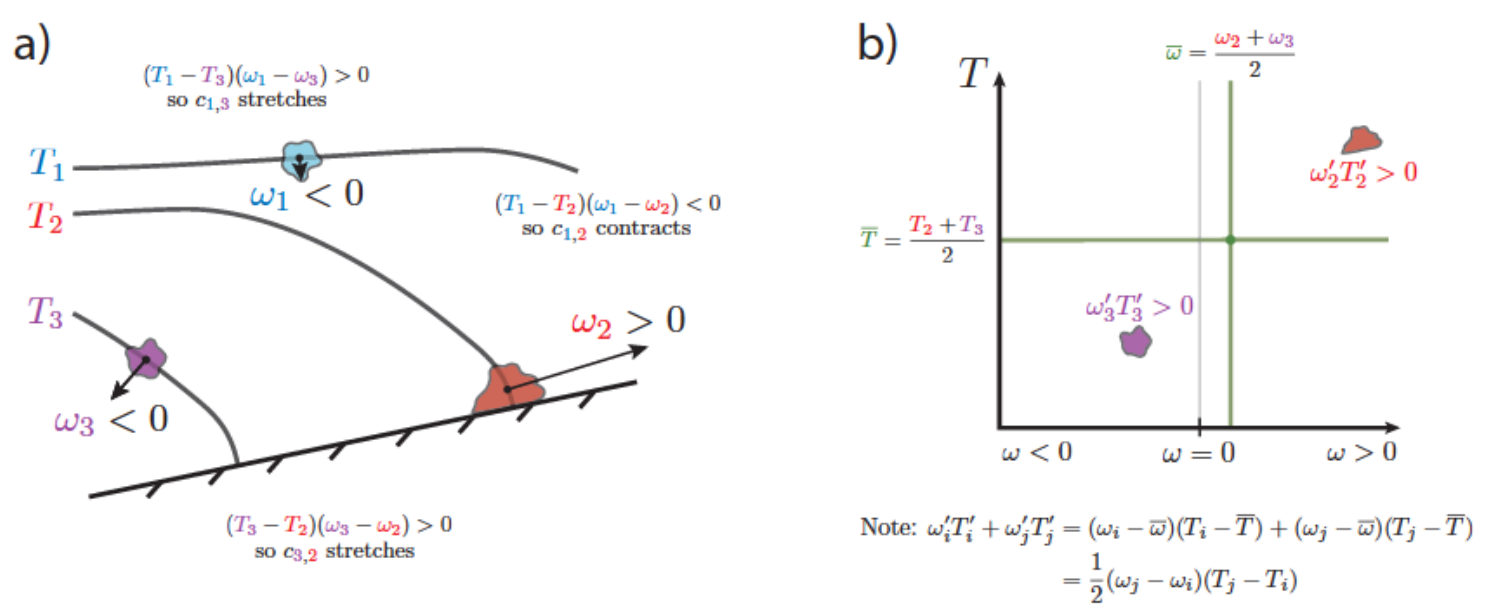

Figure 4-1: Examples of diapycnal stretching and contraction of bi-modal tracer distributions (eq. 4.5) in physical space (a) and in $(\omega, T)$ space (b). a) The three tracer distributions $c_{1,3}, c_{1,2}$, and $c_{3,2}$ experience diapycnal spreading or contraction effects due to temperature and buoyancy velocity differences. Gray lines show the equally-spaced temperature surfaces corresponding to the three tracer patches. Arrows represent the magnitude of the buoyancy velocity $\omega$ and are oriented normal to temperature surfaces. b) PDF of contributions to $c_{3,2}$ 's net diapycnal stretching effects both tracer patches. Olive lines mark the average buoyancy velocity and temperature of the tracer. 


\subsubsection{Interpreting the tracer-weighted buoyancy moments}

Consider the extreme example of a tracer distribution $c_{i, j}$ consisting of two infinitesimal patches at locations $\mathbf{x}_{i}$ and $\mathbf{x}_{j}$ and with equal mass,

$$
c_{i, j}(\mathbf{x})=\delta\left(\mathbf{x}_{i}\right)+\delta\left(\mathbf{x}_{j}\right)
$$

where $\delta(\mathbf{x})$ is the Kronecker delta. The evolution of the first moment (4.3) is simply given by the twice the average buoyancy velocity of the two patches,

$$
\partial_{t} \bar{T}=2 \bar{\omega}=\omega_{i}+\omega_{j}
$$

where we use the shorthand $\phi_{k} \stackrel{\text { def }}{\equiv} \phi\left(\mathbf{x}_{k}\right)$. The evolution of the centered second moment 4.4 , is given by

$$
\frac{1}{2} \partial_{t} \overline{(T-\bar{T})^{2}}=\overline{\kappa|\nabla T|^{2}}+\frac{1}{2} \Delta \omega \Delta T
$$

where $\Delta \omega \stackrel{\text { def }}{=} \omega_{j}-\omega_{i}$ and $\Delta T \stackrel{\text { def }}{=} T_{j}-T_{i}$ are buoyancy velocity and temperature differences between the two patches, respectively. While the first moment tendency is simply given by the average of the two patches' tendencies, the centered second moment tendency includes an additional non-linear interaction term. If the warmer patch upwells faster than the colder patch $(\Delta \omega \Delta T>0)$, this term drives diapycnal stretching (e.g. $c_{1,3}$ and $c_{3,2}$ in Figure 4-1); conversely, $\Delta \omega \Delta T<0$ corresponds to diapycnal contraction (e.g. $c_{1,2}$ in Figure 4-1).

A corollary of (4.7) is the fact that estimates of the in-situ diffusivity are most reliable when the injected tracer distribution is compact in buoyancy space (i.e. $\Delta T \approx 0$ ), an implicit assumption underlying the practice of localized TREs. Conversely, this scaling can be exploited to construct tracer distributions with arbitrarily small or large diapycnal spreading rates which have no clear relationship to the in-situ diffusivity, e.g. $\pm \kappa_{\text {Tracer }} \gg \max (\kappa)$. Even for an initially compact tracer injection, diapycnal stretching effects may become significant over time as $\Delta T$ increases due to diapycnal diffusion; on the other hand, while isopycnal stirring does not increase $\Delta T$ (by definition), it can increase $\Delta \omega$ by spreading tracer into regions with varying buoyancy velocities; while the former effect is likely to be well represented in 1D advection-diffusion models, the latter is not. 


\subsection{Numerical methods overview: simulated TREs}

We use the MITgcm to simulate mixing-driven flow in the BBTRE region, as described in detail in Chapter 3.3 After spinning up the flow to a quasi-equilibrium state at $t=5000$ days, we release three Gaussian blobs of tracer,

$$
c_{n}(x, y, z, t=0)=c_{0} \exp \left\{\frac{2}{-}\left(\frac{\left(x-x_{0}\right)^{2}}{\sigma_{x}^{2}}+\frac{\left(y-y_{0}\right)^{2}}{\sigma_{y}^{2}}+\frac{\left(z-z_{n}\right)^{2}}{\sigma_{z}^{2}}\right)\right\}
$$

with horizontal widths of $\sigma_{x}=\sigma_{y}=10 \mathrm{~km}$, a thickness $\sigma_{z}=100 \mathrm{~m}$, and horizontal release co ordinates $\left(x_{0}, y_{0}\right)$ corresponding to the oceanic BBTRE coordinates of $\left(18.3^{\circ} \mathrm{W}, 21.7^{\circ} \mathrm{S}\right)$ (Figure 4-2). The tracers are released at three different heights $z_{n}$ corresponding to distinct dynamically interesting regimes: far above the topography, $z_{\mathrm{BBTRE}}-d\left(x_{0}, y_{0}\right)=1050 \mathrm{~m}$ above the seafloor (actual BBTRE release location; hereafter the "BBTRE" tracer); roughly at the height of the canyon crests, $z_{\text {Crest }}-d\left(x_{0}, y_{0}\right)=600 \mathrm{~m}$ ("Crest"); and within the thick BBL of the canyon trough, $z_{\text {Bottom }}-d\left(x_{0}, y_{0}\right)=150 \mathrm{~m}($ "Bottom").

We follow the evolution of the released tracers until $t_{f}=440$ days after release, roughly corre sponding to the first survey in the BBTRE at 14 months. In our slope-native configuration, one should imagine infinitely many copies of TREs, each separated by a horizontal distance $L_{x}=480 \mathrm{~km}$ (domain length) and vertical height $L_{x} \tan \theta \approx 1000 \mathrm{~m}$ (corresponding to a background tempera ture difference $\Delta T \approx \frac{\mathrm{d} T_{0}}{\mathrm{~d} \tilde{z}} L_{x} \sin \theta=0.52{ }^{\circ} \mathrm{C}$ ). A limitation of this configuration is that by 1000 days enough of the tracer crosses the periodic boundaries that the copies of the tracers begin significantly interfering with each other and the temperature moment calculations become meaningless, which is why we truncate the simulations after the timing of the first BBTRE survey $(14$ months $\approx 440$ days). For all of the analysis presented here, we re-center our periodic domain on the center of mass of a single copy of the tracer cloud before adding the background state (i.e. the constantly stratified background temperature field $T_{b}$ ) back in and then we crop the infinite domain to ignore other copies of the tracer (similar to the approach used to compute watermass transformations in Chapter 3.6. 
a) Numerical simulation domain $\&$ tracer release location $\left(x_{0}, y_{0}\right)$

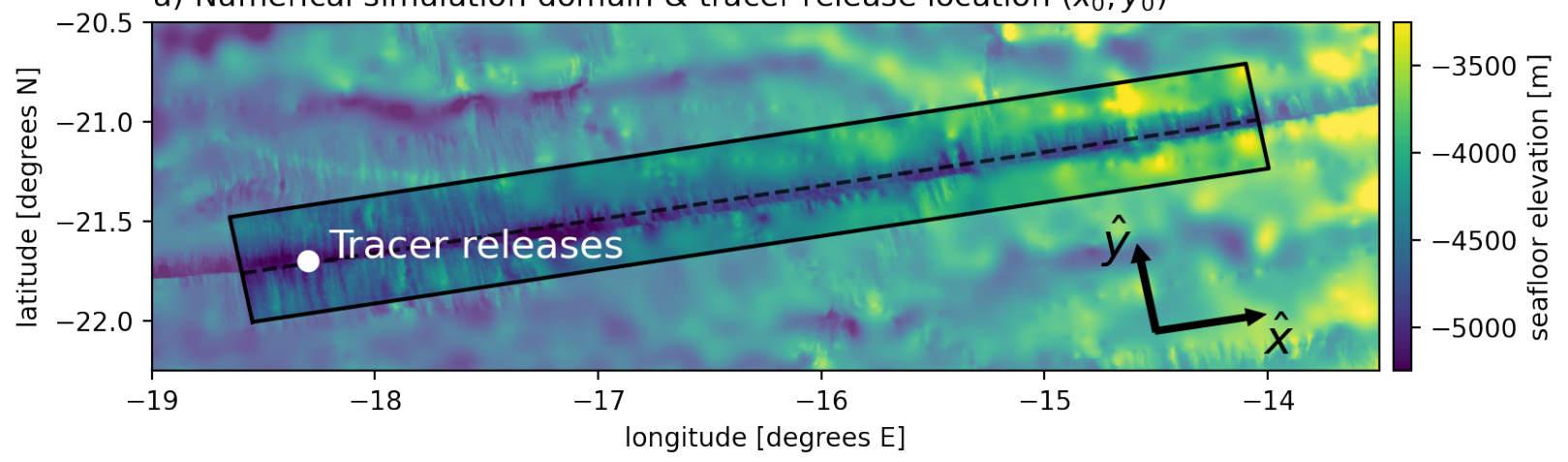

b) Prescribed bottom-enhanced mixing in a mean-slope coordinate frame

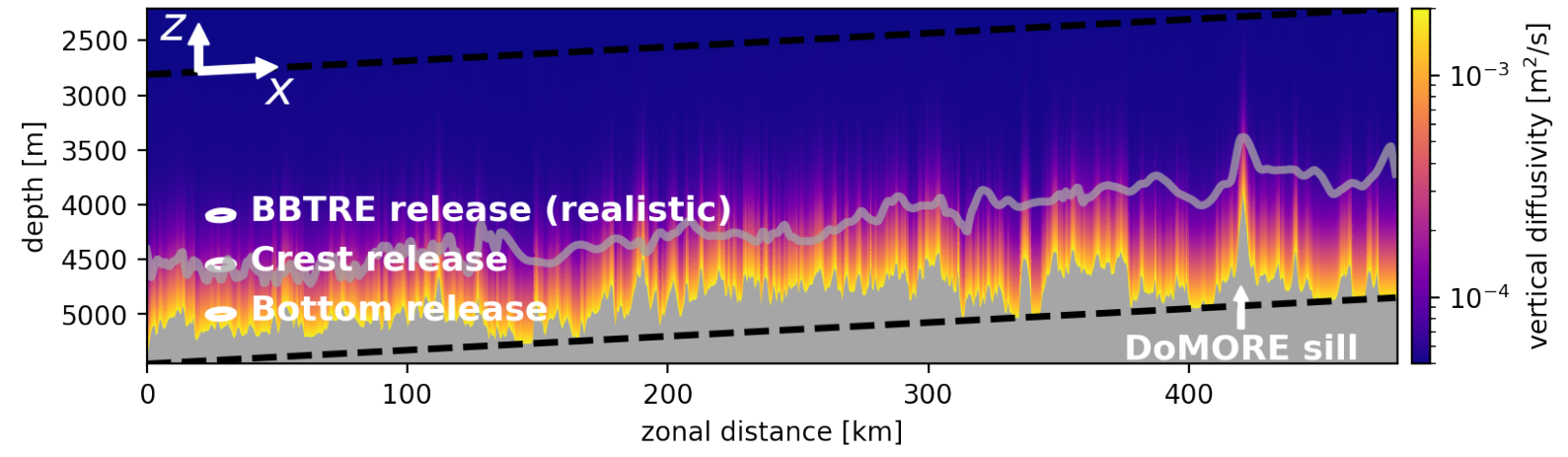

Figure 4-2: Numerical model configuration: domain geometry, prescribed forcing, and key features. a) Rectangular domain (solid lines) centered along the BBTRE canyon thalweg (dashed line) and interpolated onto locally-tangent cartesian coordinates $(\hat{x}, \hat{y}))$. b) Prescribed bottom-enhanced mix ing (colors) along the canyon thalweg (grey shading). Dashed black lines show the domain limits in the slope-native coordinate frame $(x, z)$. The transparent grey line shows the average height of the canyon crests, which rise $500 \mathrm{~m}$ to $1000 \mathrm{~m}$ above the thalweg. White dots/contours show the locations of the simulated tracer releases.

\subsection{Results}

\subsubsection{Temporal evolution of the released tracer distributions}

Within the first few eddy turnover timescales, the released tracer blobs are stirred into a web of filaments along isopycnals by submesoscale eddies (Figure 4-3a,b). While the BBTRE and Crest tracers are released well above the canyon thalweg (deepest section; Figure 4-2a), vigorous alongridge mean flow (Figure B-1) and isopycnal stirring by submesoscale eddies spread them to shallower regions (Figure 4-4).

The tracers are diffused diapycnally by the prescribed bottom-enhanced turbulent mixing (e.g. Figure 4-5b,d). For the BBTRE tracer, which mostly remains well above the bottom, its distribu tion in temperature space remains reasonably Gaussian, reminiscent of diffusion with a constant 

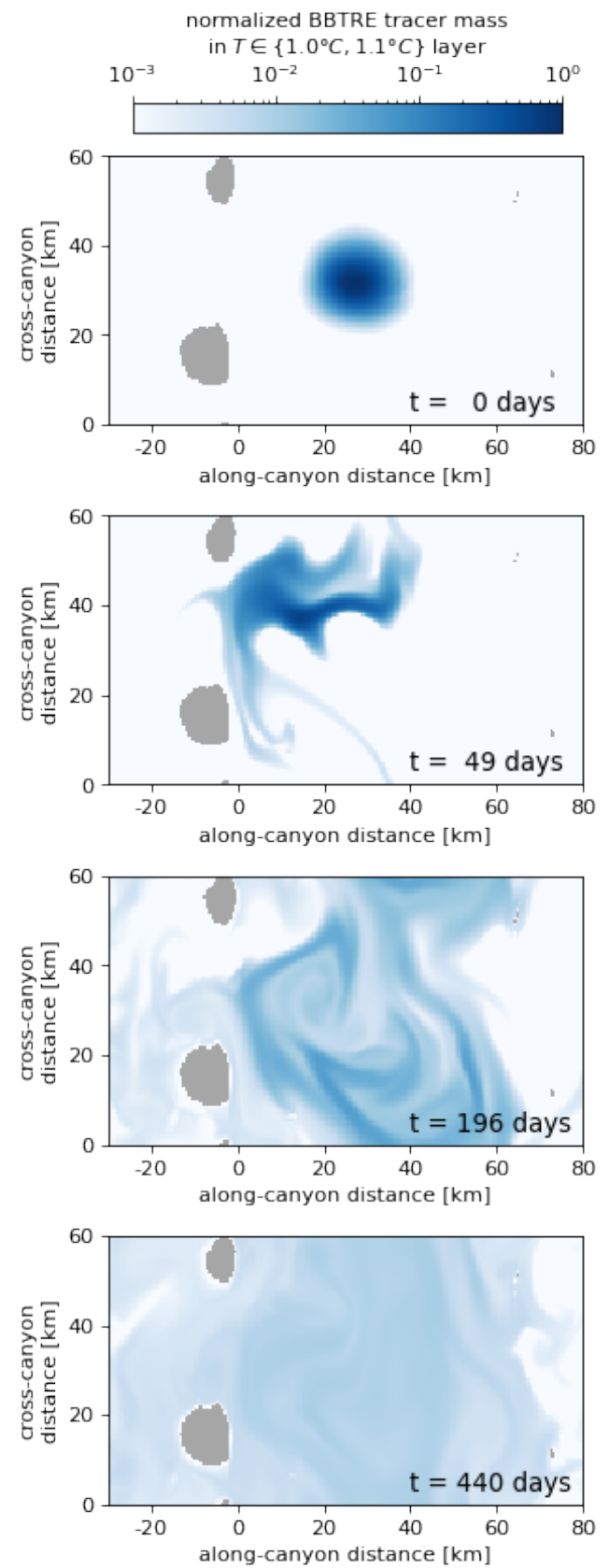

Figure 4-3: Instantaneous snapshots of the BBTRE tracer mass, vertically-integrated over the $\left\{1.0^{\mathrm{O}} \mathrm{C}, 1.1^{\mathrm{O}} \mathrm{C}\right\}$ temperature layer, and normalized by the maximum initial tracer mass. The grey shading represents two major topographic obstacles, where the temperature layer in-crops.

diffusivity and in the absence of boundaries; for the Crest and Bottom tracers, however, the tracer distributions depart significantly from Gaussianity (Figure 4-5h,l), suggesting the importance of variations in the diffusivity or boundary effects. Most notably, the Bottom tracer develops a bi modal distribution in temperature space as some of the tracer spills over the sill at $x=120 \mathrm{~km}$ 


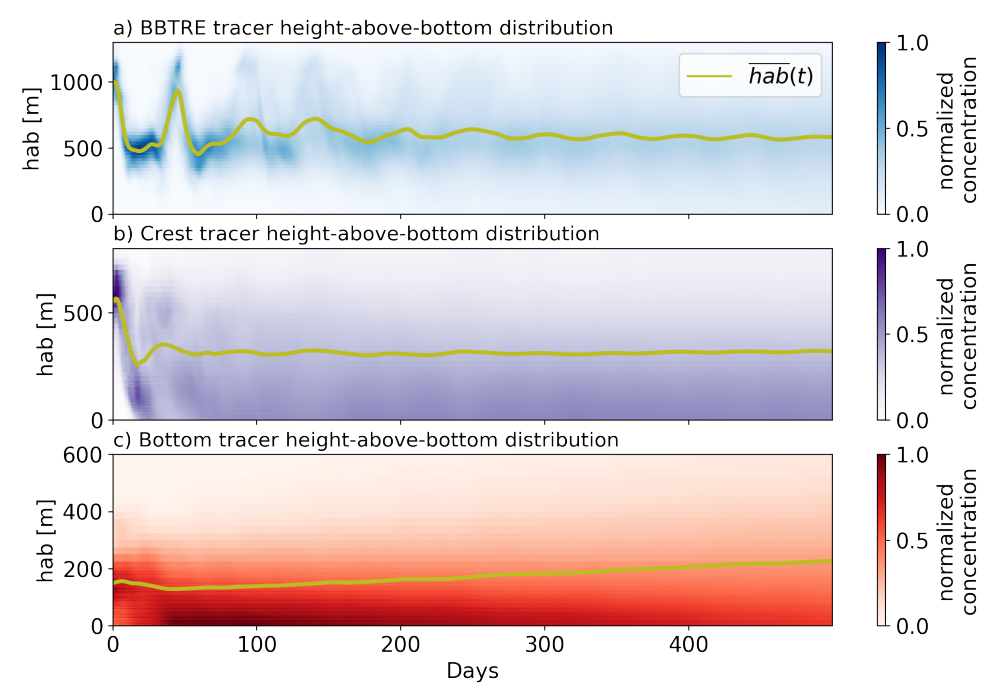

Figure 4-4: Height above bottom distributions of the three tracers over time, normalized by the maximum bin value. Olive lines show the trajectory of the average height-above-bottom of the tracer distribution.

and crosses the $T=0.7^{\circ} \mathrm{C}$ surface (Figure 4-5j, l). By the end of the experiment at 440 days, most of the Bottom tracer has spilled over the sill and its bi-modal distribution collapses onto a single, warmer, peak. While only the Bottom tracer exhibits a tracer-weighted diapycnal motion that is discernible by visually inspecting the tracer distributions in temperature space, the BBTRE and Crest tracers do exhibit slow mean diapycnal downwelling and upwelling, respectively.

\subsubsection{Diapycnal interior downwelling and near-boundary upwelling driven by bottom-enhanced mixing}

As described in Section 4.2.1, the mean diapycnal motion of the tracer is directly driven by the tracer-weighted buoyancy velocity $\bar{\omega} \stackrel{\text { def }}{\equiv} \overline{\nabla \cdot(\kappa \nabla T)}$ (eq. 4.3). In the SML, well above the seafloor, the bottom-enhancement of the diffusivity $\kappa$ dominates the buoyancy velocity, resulting in diapycnal downwelling, $\omega<0$ (Figure 4-7a; grey line). Closer to the seafloor, the temperature flux must vanish to satisfy the insulating bottom boundary condition, resulting in vigorous diapycnal upwelling, $\omega>0$, in the BBL (Figure 4-7a; grey line). Thus, by definition a tracer entirely in the SML will sink, while a tracer entirely in the BBL will upwell. Since the upwelling velocity in the BBL is roughly an order of magnitude larger than the maximum downwelling velocity in the SML, at least ten times as much tracer must be in the SML than in the BBL for the tracer to downwell on average.

The BBTRE tracer, which is released in the SML, exhibits diapycnal downwelling throughout the experiment (Figure 4-6.), consistent with the heuristic argument above. The magnitude of 

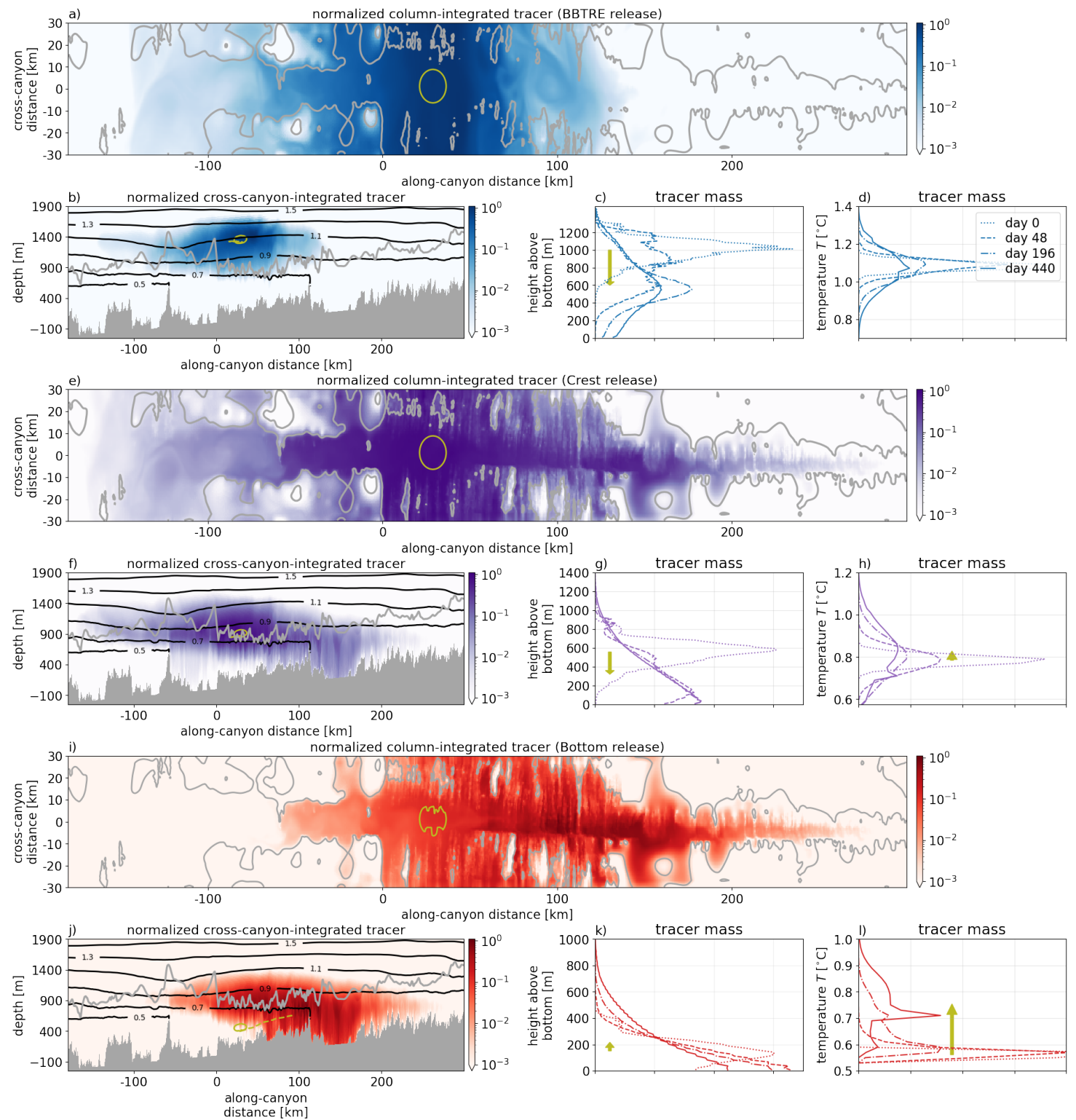

Figure 4-5: Temporal evolution of the tracer distributions in Cartesian, height-above-bottom, and temperature coordinates for the BBTRE (a-d; blues), Crest (e-h; purples), and Bottom releases (i-l; reds). (a,e,i) Slope-normal column-integrated tracer concentrations and $(\mathrm{b}, \mathrm{f}, \mathrm{j})$ cross-canyon integrated tracer concentrations, normalized by their maximum value (logarithmic scale). Grey contours in $(\mathrm{a}, \mathrm{e}, \mathrm{f})$ show two representative isobaths of ocean depth $\hat{d}(\hat{x}, \hat{y})$. Black lines in $(\mathrm{b}, \mathrm{f}, \mathrm{j})$ show equally-spaced cross-canyon-averaged temperature surfaces. Tracer distributions in (c,g,k) height-above-bottom and $(\mathrm{d}, \mathrm{h}, \mathrm{l})$ temperature coordinates, normalized by their maximum initial values (linear scale). Olive contours show the $10 \%$ contour for the initial tracer distribution; olive dashed and arrows show the temporal evolution of the tracer's center of mass (first moments).

downwelling, however, is modulated by a 45-day damped oscillation due to along-ridge advection by a mean-flow of speed $U \approx 15 \mathrm{~mm} / \mathrm{s}$ (Figure B-1) across a periodic domain of width $L_{y}=60 \mathrm{~km}$, such that the tracer aliases the roughly sinusoidal trough-crest topography on a timescale of $\tau=L_{y} / U \approx$ 
45 days (Figure B-1). This modulation is damped over time as the tracer spreads isopycnally and spans a region wider than the typical trough-crest separation (Figure 4-5a).

In the first 100 days, interpreting the mean diapycnal sinking of the BBTRE tracer is straight forward: the entire tracer distribution experiences a negative buoyancy velocity and so the tracer sinks diapycnally (Figure 4-6 c), i.e. $\bar{\omega} \approx \overline{\omega_{<0}}$, where we define:

$$
\overline{\omega_{<0}} \stackrel{\text { def }}{\equiv} \frac{\int_{\mathcal{V}_{\omega<0}} \omega c \mathrm{~d} V}{\int_{\mathcal{V}} c \mathrm{~d} V}
$$

as the strictly downwelling buoyancy velocity. While this strictly downwelling contribution to the tracer-weighted buoyancy velocity increases slightly over the remainder of the experiment as the tracer sinks towards larger diffusivities, sufficient tracer is entrained into the BBL that a strictly upwelling contribution $\overline{\omega_{>0}}$ (similarly defined) grows at an even faster rate, such that the net diapycnal sinking of the tracer weakens over the last few hundred days of the experiment.

At the other extreme, the Bottom tracer is released entirely in the BBL and thus upwells vigorously upon release, with $\bar{\omega} \approx \overline{\omega_{>0}}$ (Figure $4-6 \mathrm{~g}$ ). As some of the tracer eventually spreads into the SML above and the strictly negative contribution $\overline{\omega_{<0}}$ grows, the net upwelling of the tracer weakens over time 1 .

The Crest release is perhaps the most interesting: at first, the Crest tracer is in the SML far above the canyon thalweg and thus downwells similarly to the BBTRE tracer; after roughly $\tau=46$ days, however, enough of the Crest tracer is advected into the BBLs along the crest of the canyon that the strictly upwelling component wins out and the tracer begins upwelling in the net (Figure 4-6). Over the last few hundred days of the experiment, the weak net upwelling of the tracer is the small residual of a substantial compensation between strictly upwelling and strictly downwelling contributions.

Plotting the evolution of the temperature moment tendency as a function of the tracer-weighted height-above-bottom, we recover a blurred view of the height-above-bottom structure of the in-situ buoyancy velocity (Figure 4-7 a, colored circles). In practice, however, only a time-averaged view of the first temperature moment tendencies is possible: time-averaged tendencies are calculated by differencing snapshots of a tracer moments at two different times (i.e. by integrating eq. 4.3 , $\left.\frac{1}{t_{1}-t_{0}} \int_{t_{0}}^{t_{1}} \partial_{t} \bar{T} \mathrm{dt}=\frac{\bar{T}\left(t_{1}\right)-\bar{T}\left(t_{0}\right)}{t_{1}-t_{0}}\right)$, and provide an even more coarse-grained view of the in-situ vertical

\footnotetext{
${ }^{1}$ The Bottom tracer is released near the bottom of a weakly stratified depression along the canyon thalweg, and its average stratification increases dramatically over the first 200 days (Figure 4-6a,b). Thus, the early diapycnal upwelling and spreading is enhanced when converting to physical space because the buoyancy surfaces are on average much further apart than they are later on (see normalization in equations 4.3 and 4.4 and Figure B-2.
} 
structure than that provided by many instantaneous snapshots of the first moment tendencies (Figure 4-7a; stars).

\subsubsection{Bottom-enhanced diapycnal tracer spreading}

Over the course of the experiment, all three tracers spread across isopycnals on average. As antici pated from the prescribed bottom-enhanced diffusivity profile, the closer a tracer is released to the bottom the faster it spreads (Figure 4-7 a; stars). However, this time-mean view betrays a surprising temporal variability (Figure 4-6, h). This tendency is particularly dramatic for the Bottom tracer, which experiences extremely rapid diapycnal spreading in the first 150 days but, by day 350, stops spreading entirely and even begins temporarily contracting in buoyancy space (Figure 4-6h)! The interpretation of diapycnal tracer spreading is more subtle than that of the mean diapycnal motion of the tracer, since two separate terms contribute to spreading: the tracer-weighted effective dif fusivity $\kappa_{\text {Taylor }}$ and diapycnal stretching $\kappa_{\omega}$ (eq. 4.4). The contribution from the tracer-weighted effective diffusivity is familiar from Taylor (1922)'s classic derivation, and is well approximated by the tracer-weighted in-situ diffusivity $\bar{\kappa}$ since tracer-weighted variations in the squared temperature gradients are small (Figure $4-6 \mathrm{~d}, \mathrm{f}, \mathrm{h} ; \mathrm{B}-2 \mathrm{~b}, \mathrm{~d}, \mathrm{f}$ ),

$$
\kappa_{\text {Taylor }} \stackrel{\text { def }}{=} \frac{\overline{\kappa|\nabla T|^{2}}}{\overline{|\nabla T|^{2}}} \approx \bar{\kappa} .
$$

This contribution to diapycnal spreading from the tracer-weighted diffusivity remains roughly con stant in time, aside from an initial transient as the tracer spreads towards shallower topographic features (Figures 4-7b and 4-4). In contrast, the diapycnal stretching effect drives the substantial temporal variability in the diapycnal spreading experienced by the tracers (Figure 4-6d,f). De pending on the instantaneous distribution of the tracer, this term can vary substantially both in magnitude and sign, either amplifying the tracer-weighted diffusivity by up to $100 \%$ or off-setting it entirely (Figure 4-7b,c and B-2; at 40 and 400 days, respectively).

\subsubsection{Disentangling diapycnal stretching and contraction effects}

Motivated by the bi-modal example in Section 4.2.2, we decompose the diapycnal stretching term

$2 \overline{\omega^{\prime} T^{\prime}}$ by binning its contributions in $(\omega, T)$ space (Figure 4-8, as in Figure 4-1 b), i.e. decomposing 

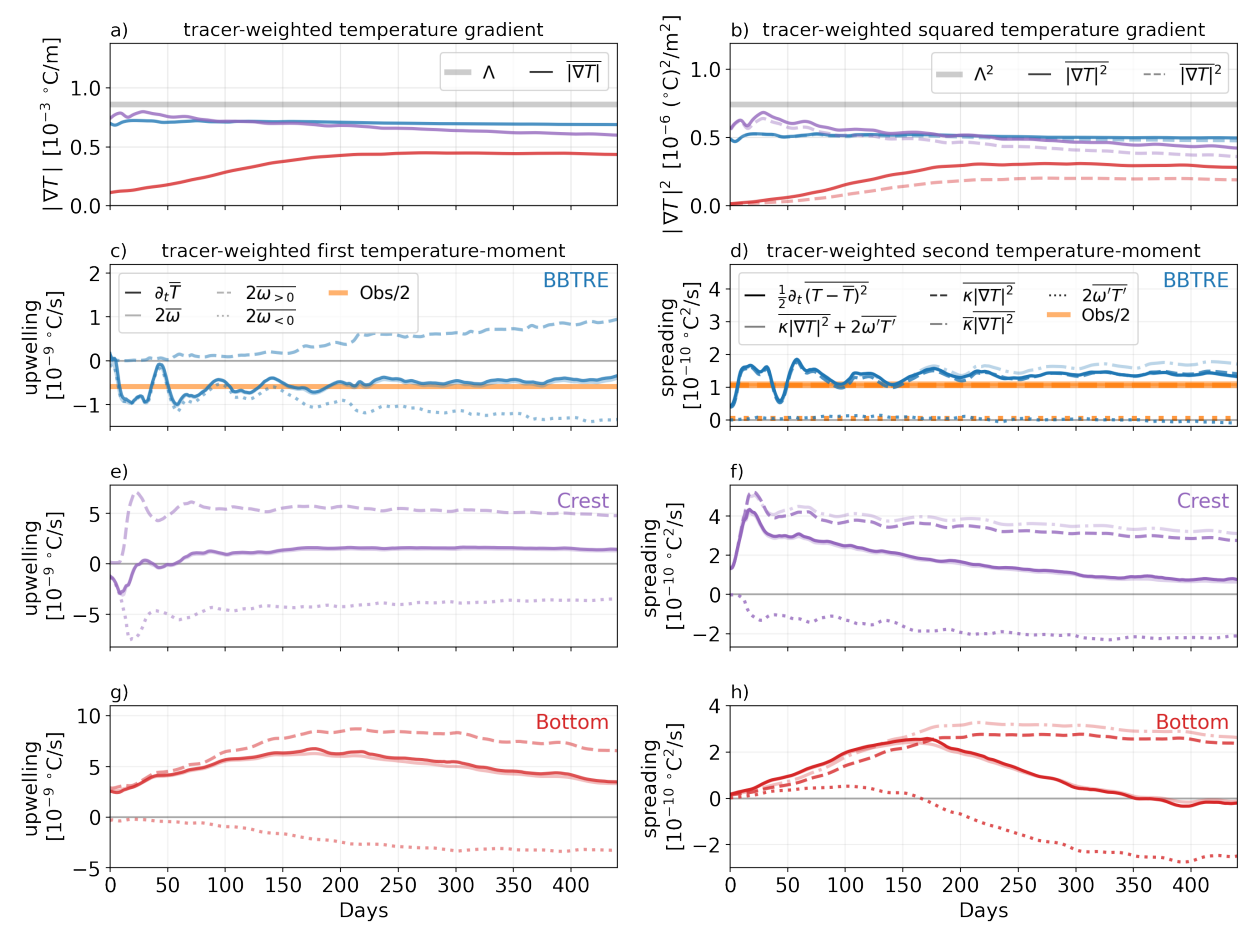

Figure 4-6: Decomposition of the first and second tracer-weighted temperature moment tendencies, i.e. the rates of diapycnal motion and spreading, in terms of their driving mixing processes. The realistic BBTRE release is shown in blue, the Crest release in purple, and the Bottom release in red. (a-b) show the temporal evolution of the tracer-weighted stratification and stratification squared, with the background stratification $\Lambda \stackrel{\text { def }}{\equiv} \frac{\mathrm{d} T_{b}}{\mathrm{~d} \tilde{z}}$ for reference. (c-h) show the temporal evolution of the first and second moment tendencies (opaque solid lines), and the sum of the contributing mixing processes (transparent solid lines), as described by the left- and right-hand-sides of equations (4.3), $\partial_{t} \bar{T}=2 \bar{\omega}$, and $(4.4), \frac{1}{2} \partial_{t} \overline{(T-\bar{T})^{2}}=\overline{\kappa|\nabla T|^{2}}+2 \overline{\omega^{\prime} T^{\prime}}$, respectively. Orange lines in (c,d) show the time-averaged BBTRE moment tendencies from observations, reduced by a factor of 2 for ease of comparison. (c,e,g) Dashed and dotted lines show the contributions from strictly upwelling and strictly downwelling regions, respectively. (d,f,h) Dashed lines show the contribution from tracerweighted in-situ diffusion $\kappa_{\text {Taylor }}$ (approximately equal to the tracer-weighted in-situ diffusivity $\bar{\kappa}$; dash-dotted lines) and dotted lines show the contribution from diapycnal stretching $\kappa_{\omega}$.

the sum

$$
2 \overline{\omega^{\prime} T^{\prime}}=\sum_{i, j}\left(2 \frac{\int_{\mathcal{V}_{\left\{\omega_{i}\right\} \cap\left\{T_{j}\right\}}} \omega^{\prime} T^{\prime} c \mathrm{~d} V}{\int c \mathrm{~d} V}\right) / \overline{|\nabla T|^{2}},
$$

where the intersection of subsets $\left\{\omega_{i}\right\}$ and $\left\{T_{j}\right\}$ correspond to distinct sub-volumes $\mathcal{V}_{\left\{\omega_{i}\right\},\left\{T_{j}\right\}}$ of the tracer distribution (e.g. where $T>\bar{T}$ ). Throughout this section, we quantify diapycnal stretching in terms of percentages of the effective diapycnal diffusion $\kappa_{\text {Taylor }}$ (i.e., we normalize eq. 4.11 by $\left.\kappa_{\text {Taylor }}\right)$, where positive percentages denote stretching and negative percentages denote contraction.

We begin by exploring why the BBTRE tracer experiences very little net diapycnal stretching, 

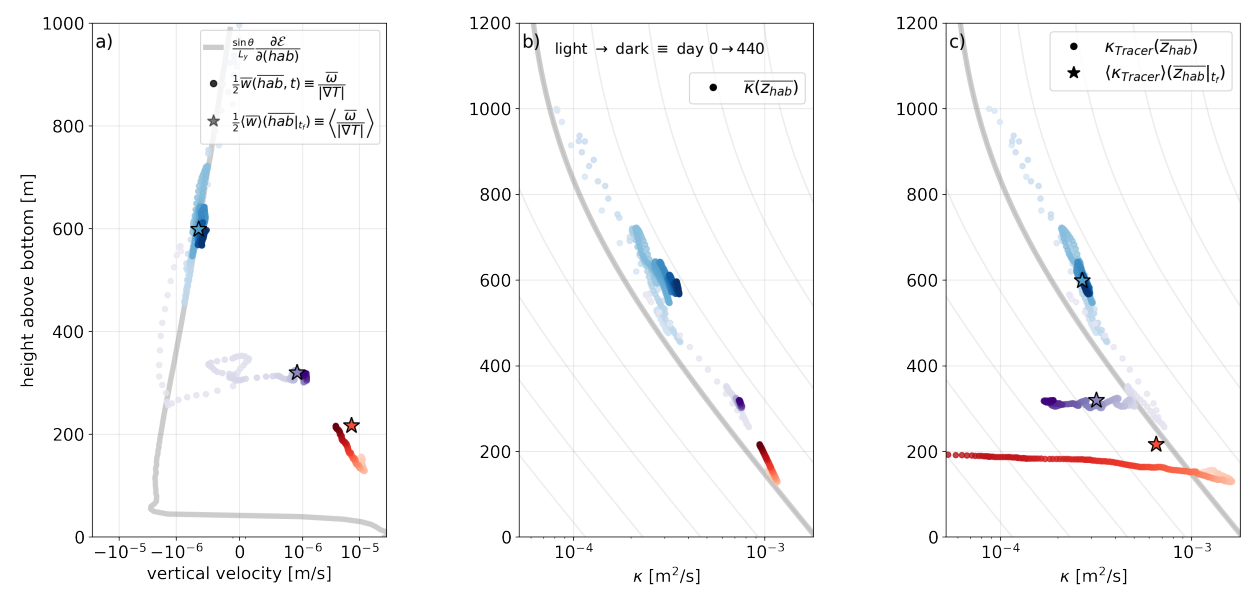

Figure 4-7: (a) Height-above-bottom-averaged buoyancy velocity (grey) and tracer-weighted buoy ancy velocity (colors). (b,c) As in (a), but for the tracer-weighted in-situ diffusivity $\bar{\kappa}$ and tracer diffusivity $\kappa_{\text {Tracer }}$. Dots show trajectories (colors darken over time) in terms of the magnitude of moment tendency terms and the tracer-weighted height-above-bottom $(\overline{\mathrm{hab}}(t))$. Stars in (a,c) show the time-averaged moments, as they would be estimated from a TRE survey 440 days after release (assuming perfect spatial coverage).

in contrast to the idealized SML simulation of Ruan and Ferrari (2021). At day 100 (Figure 4-8b), for example, the tracer is diapycnally stretched $\left(\overline{\omega^{\prime} T^{\prime}}>0\right)$ by an additional $23 \% \kappa_{\text {Taylor }}$ as relatively cold tracer relatively downwells $\left(T^{\prime}<0, \omega^{\prime}<0\right)$ and relatively warm tracer relatively upwells $\left(T^{\prime}>0, \omega^{\prime}>0\right)$, so far consistent with Ruan and Ferrari (2021)'s idealized result of $18 \%$ additional stretching. However, a very small amount of cold tracer has made it close enough to the seafloor to be entrained in the BBL, where it upwells vigorously and results in a contraction effect of $-9 \%$ which, supplemented by an additional $-2 \%$ contraction from warm downwelling tracer, results in a reduction of the net diapycnal stretching to only $12 \%$. By day 440 , this patch of tracer is pulled further towards the bottom and its stretching effect grows to $69 \%$, but is offset by an even larger diapycnal contraction of $-76 \%$ in the BBL (Figure 4-8c); combined, these diapycnal stretching effects have a negligible effect of reducing diapycnal spreading by only $-6 \%$.

At the other extreme, we aim to understand how the Bottom tracer undergoes first a large net diapycnal stretching effect and then an even larger net diapycnal contraction effect. Over the first 100 days, most of the tracer upwells in the BBL and warms (Figure 4-6s). Some of the warmest tracer remains in the BBL, where its upwelling drives a substantial diapycnal stretching of $91 \%$ (Figure 4-8a). However, another relatively warm branch of the tracer is entrained into the SML, where its downwelling drives a largely compensating diapycnal contraction effect of $-80 \%$. The relatively cold patch of tracer that is left behind contributes a stretching of $41 \%-15 \%=26 \%$, 

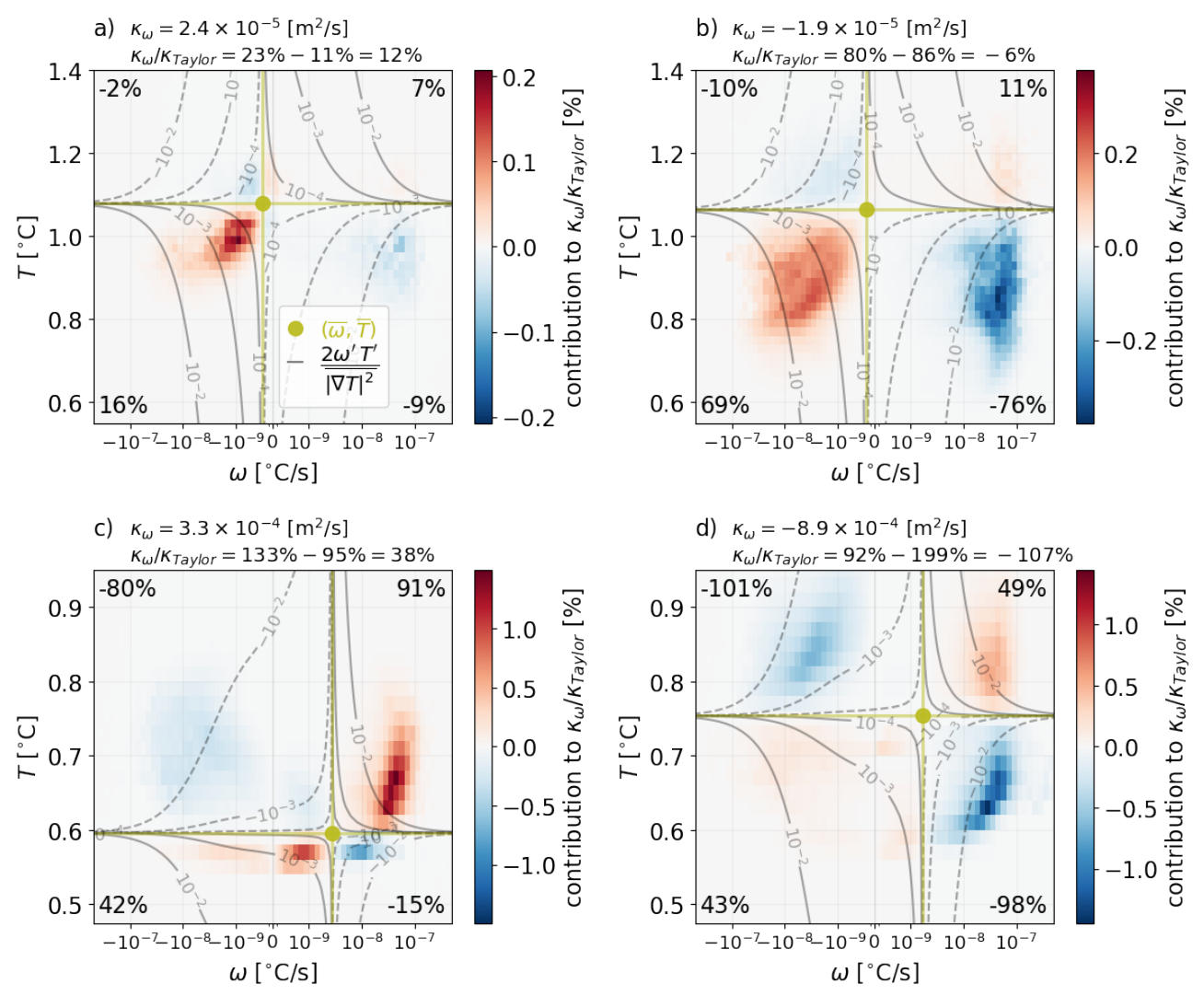

Figure 4-8: Percentage contributions to the net diapycnal stretching, effect (eq. 4.11, normalized by $\left.\kappa_{\text {Taylor }}\right)$ from each $(\omega, T)$ bin, as a percentage, for the BBTRE (top) and Bottom (bottom) tracers at 100 days (left) and 440 days (right). Numbers in the four corners of each panel show the total contribution from each of the four quadrants defined by the signs of $\omega^{\prime} \stackrel{\text { def }}{\equiv} \omega-\bar{\omega}$ and $T^{\prime} \stackrel{\text { def }}{\equiv} T-\bar{T}$, expressed as a percentage of the effective in-situ diffusivity, $\kappa_{\text {Taylor }}$. The $\omega^{\prime}=0$ and $T^{\prime}=0$ lines in olive delineate the four quadrants. For reference, grey contours show the effective diapycnal stretching diffusivity, $\kappa_{\omega} \stackrel{\text { def }}{=} 2 \frac{\overline{\omega^{\prime} T^{\prime}}}{\mid \overline{\left.\nabla T\right|^{2}}}$, that corresponds to each $(\omega, T)$ bin. Sub-titles decompose the net diapycnal stretching effect into strictly stretching $\left(\omega^{\prime} T^{\prime}>0\right)$ and strictly contracting components $\left(\omega^{\prime} T^{\prime}<0\right)$. PDFs of tracer mass show that a large portion of the tracers' mass does not contribute significantly to these stretching effects (Figure B-3).

dominated by its relatively slow upwelling $\left(\omega>0\right.$ but $\left.\omega^{\prime}<0\right)$, bringing the net diapycnal stretching to $91 \%-81 \%+26 \%=37 \%$. By day 440 , however, both modes of the tracer distribution (Figure 4-5.) drive large diapycnal contraction effects: $-98 \%$ due to cold upwelling upstream of the sill and $-101 \%$ due to warm downwelling downstream of the sill (Figures 4-5,, $14-8$ ). Diapycnal stretching of $93 \%$ from the other quadrants offset about half of this diapycnal stretching effect, but the net diapycnal contraction of $-106 \%$ still overwhelms the spreading due to the in-situ diffusivity, causing the Bottom tracer to temporarily contract in buoyancy space. 


\subsection{Discussion and Conclusion}

By applying Ruan and Ferrari (2021)'s buoyancy-moment diagnostics to our quasi-realistic regional simulation of mixing-driven abyssal flows (described in detail in Chapter 3), we confirm the qualita tive results of Holmes et al. (2019)'s idealized analysis of the BBTRE release in the SML: over time, boundary suppression in the BBL almost exactly compensates for vertical stretching in the SML, such that the net diapycnal spreading of the BBTRE tracer coincidentally provides a reasonably accurate $( \pm 20 \%)$ estimate of the tracer-weighted in-situ diffusivity (Figure 4-6d). These simulation results are supported by a recent re-analysis of the BBTRE observations, which reveal a similarly negligible diapycnal stretching effect of $<5 \% \kappa_{\text {Taylor }}$ (Ledwell, in prep). Quantitatively, however, the diapycnal spreading we simulate for the BBTRE tracer is smaller than the observed spreading by a factor of 2 (Figures 4-6 d, 4-9), suggesting either the microstructure measurements we use to tune the prescribed diffusivity profile are biased low or our simulation is missing other unknown tracer dispersion processes. This is consistent with Ledwell (in prep)'s inversion of a 1D advection diffusion model, which produced optimal diffusivities about twice as large as the microstructure's sample-mean. These results are consistent with our conclusion from Chapter 3 that biases in the simulated flows and stratification of the BBTRE fracture zone canyon are consistent with the im posed microstructure mixing rates being biased too low by a factor of roughly 2. They are also consistent with the broader observational literature, which unanimously finds that mixing rates estimated from TREs are larger than those suggested by co-located microstructure measurements (Ruan and Ferrari, 2021).

In contrast to the BBTRE release, we find that for near-bottom tracers diapycnal stretching/contraction effects can be of either sign (depending on the tracer distribution) and of com parable magnitude to the tracer-weighted in-situ diffusivity, $\left|\kappa_{\omega}\right| \sim \bar{\kappa}$ (Figure 4-6h). Boundary suppression of diapycnal spreading, in particular, is even more dramatic in our simulation with real istically rough topography than in Holmes et al. (2019)'s model of tracer transport over a smoothly sloping bottom: for the Bottom tracer release, diapycnal stretching amplifies diapycnal spreading by $\mathcal{O}\left(100 \% \kappa_{\text {Taylor }}\right)$ in the first few dozen days of the simulation but suppresses diapycnal spreading by $\mathcal{O}\left(-100 \% \kappa_{\text {Taylor }}\right)$ for the last few dozen days (Figures $4-6 \mathrm{~h} ; 4-7 \mathrm{k} ; \mathrm{B}-2$; $)$. It has long been appre ciated that the interpretation of tracer spreading near topography requires greater care because of enhanced boundary mixing and hypsometric effects (e.g. Ledwell and Hickey, 1995). Attempts to modify the conventional 1D model to include these boundary effects are varied: by separating the 


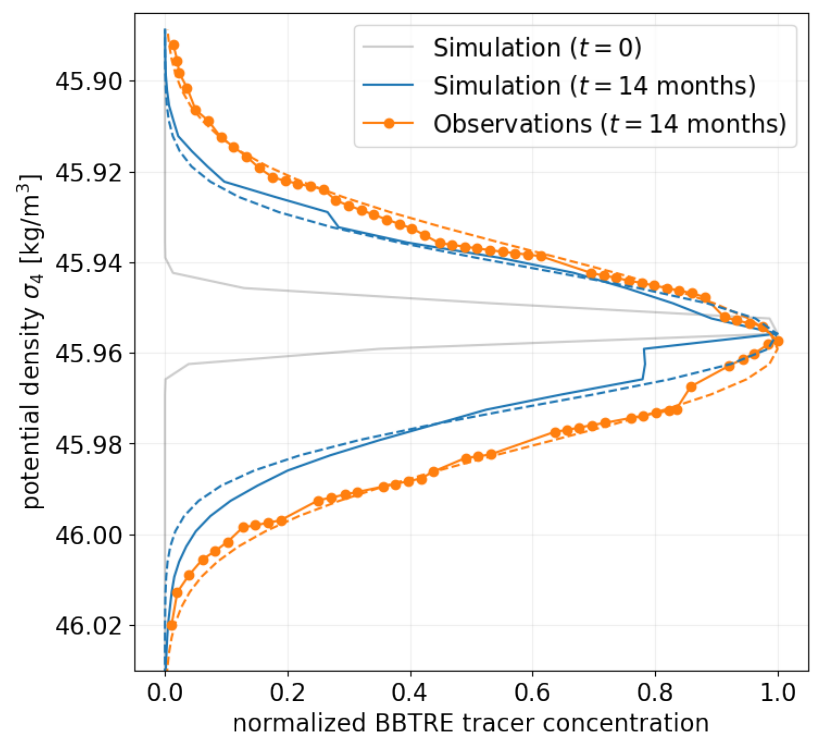

Figure 4-9: Simulated and observed tracer distributions in density space at 14 months. We fit a Gaussian distribution $\propto \exp \left\{\frac{-\left(\sigma_{4}-\sigma_{4,0}\right)^{2}}{\tilde{\kappa} t}\right\}$ to the observations by eye (dashed orange). The simulated distribution is reasonably well fit by a Gaussian distribution corresponding to a diffusivity reduced by a factor of 2 (dashed blue), consistent with the results of the buoyancy moments method (Figure 4-6, d).

tracer distribution into "boundary" and "interior" regions (Ledwell and Hickey, 1995, Ledwell et al., 2016), by allowing vertical structure in the diffusivity profile (Ledwell et al., 2000), or by extending the 1D model to a 2D (Watson et al., 2013) or 3D (Mackay et al., 2018) model to account for lateral transport into regions of strong mixing. However, the ad-hoc derivations of these models render them difficult to interpret and compare, suggesting the more general buoyancy-moment approach may prove more fruitful (Ruan and Ferrari, 2021).

Mesoscale/submesoscale-resolving regional simulations of TREs are now feasible thanks to ex ponential increases in computational power (Tulloch et al. 2014; Mashayek et al. 2017a; Ogden et al., in prep; this study) and have been used a posteriori to help interpret observations and explain differences between mixing rates inferred from TREs and microstructure profiles. However, such simulations have not yet been used to evaluate (or improve upon) operational methods for compar ing tracer and microstructure observations, such as by using a "perfect model" framework in which simulated tracer observations are inverted in an attempt to recover the prescribed "true" diffusivity field. Similarly, a priori or real-time numerical simulations could be used to inform future TRE sampling strategies (current best practice is to use real-time velocity estimates from ADCPs or altimetry; Ledwell, personal communication). To our knowledge, this has not yet been done, with 
the notable exception of Ruan et al. (in prep), who have simulated an upcoming Tracer Release Experiment and are advising the release and sampling strategies.

While our results suggest that estimates of in-situ diffusivity profiles from observations of a tracer's second buoyancy moment may be corrupted by complicated diapycnal stretching processes (Figure 4-7k; consistent with Holmes et al. 2019), they also suggest that the first buoyancy moment provides a robust and straight-forward estimate of the tracer-weighted in-situ turbulent buoyancy flux convergence (or buoyancy velocity $\omega$; Figure 4-7 a). As long as a sizable fraction (here $\gg$ $w_{\mathrm{SML}} / w_{\mathrm{BBL}} \approx 10 \%$; Figure $\left.4-7 \mathrm{a}\right)$ of the tracer remains in the BBL, the change in the first tracerweighted buoyancy-moment is likely to provide a reasonable lower-bound estimate of the average insitu buoyancy velocity in the BBL (Section 4.4.2). This is a promising result in light of the upcoming Bottom Boundary Layer Turbulence and Abyssal Recipes experiment (NSF Award \#1756251), which will attempt to estimate the BBL buoyancy velocity from TREs. In combination with previous observations of tracer-weighted diapycnal sinking in the SML above rough topography (Ledwell et al. 2000), observations of vigorous tracer-weighted diapycnal upwelling in the BBL would be compelling direct evidence for the emerging paradigm of bottom mixing layer control of the abyssal meridional overturning circulation (Ferrari et al., 2016; de Lavergne et al., 2016b; Callies and Ferrari, 2018a b; Drake et al., 2020a). 


\section{Chapter 5}

\section{Outlook}

Until the processes giving rise to [near-boundary] diffusion and advection are understood, the resulting differential equations governing the interior distribution, and their solutions, must remain what they have been for so long: a set of recipes.

Walter $\longdiv { \text { Munk } ( 1 9 6 6 ) }$

Below, I discuss implications of this work and some directions for future research on the topic.

\subsection{Observational evidence of mixing-driven bottom boundary layer upwelling}

The emerging paradigm of Bottom Boundary Layer (BBL) control on the abyssal overturning, as argued in this thesis, predicts extremely large upwelling transports in a thin BBL; major criticisms of the theory (aside from those addressed by this thesis) are that 1) this predicted large diapycnal BBL upwelling is not yet falsifiable with available observations and 2) it is inferred from uncertain parameterizations of the vertical turbulent buoyancy flux. Such parameterizations are sensitive to assumptions about the underlying dynamics (e.g. the fraction of internal waves which break near their generation site; Cimoli et al. 2019) and whose underlying assumptions may break down in weakly-stratified bottom boundary layers (e.g. assuming a constant mixing efficiency; Mashayek et al. 2017b), such that their magnitudes may have uncertainties of up to a factor of 2-3 (Ferrari et al., 2016; de Lavergne et al., 2016b; Cimoli et al., 2019).

The upcoming Bottom Boundary Layer Turbulence and Abyssal Recipes field campaign (or "BLT"; NSF Award \#1756251) aims to make the first comprehensive observations of abyssal bot 
tom boundary layer turbulence. Both the presence of a rough boundary and weak stratification raise questions about the adequacy of turbulence parameterizations that require assumptions about homogeneity, isotropy, or the "efficiency" of mixing (see Gregg et al. 2018). Thus, the plan for BLT is to use a plethora of methods to estimate the mixing as close to the bottom as possible: both shear and temperature microstructure, direct (but noisy) measurements of the turbulence flux, and localized releases of both dye (short-term) and tracer (long-term) followed by surveys of their threedimensional distributions. The hope is that these observations will not only provide the first reliable constraints on the near-boundary structure and magnitude of the turbulent buoyancy flux, but also that the observed average buoyancy gain of the released dyes and tracer will be generally consistent with the inferred buoyancy flux convergence.

One reason that $\mathrm{BBL}$ upwelling transports have not yet been directly observed is that the diapycnal velocity $\mathbf{e}$ is much smaller than the isopycnal velocities associated with adiabatic mean flows and geostrophic turbulence, so small errors in the projection of the measured components onto the diapycnal direction can corrupt the estimates. Even where isopycnals are roughly flat and $\mathbf{e} \simeq w \hat{z}$, estimates of the vertical velocity are small, noisy, and easily tainted by small errors in the instruments' pitch angle relative to gravity. Thurnherr et al. (2005) attempt to circumvent this problem by considering the heat budget for a single fracture zone canyon: observed alongcanyon flows enter a volume confined by the canyon trough and sloping flanks from below and thus must upwell across the bounding buoyancy surface above; however, they are unable to find sufficient mixing to quantitatively close the canyon's buoyancy budget (Thurnherr et al., 2020). Similar heat budgets in other regions (e.g. Hogg et al., 1982; Spingys et al., 2021) follow similar arguments, but the mean diapycnal upwelling is always inferred, never directly observed. Tracers naturally provide more reliable estimates of diapycnal velocities since they inherently integrate over spatial and temporal variability. In Chapter 4, for example, I re-interpret the slow tracer-weighted diapycnal sinking observed in Brazil Basin (Ledwell et al., 2000) as direct evidence of mixing-driven downwelling in the $\mathrm{SML}^{1}$

Tracer-based estimates of mixing rates and transports are typically provided by fitting surveyed tracer concentrations to relatively ad-hoc tracer advection-diffusion models. In contrast, the tracerweighted buoyancy moment method, proposed by Ruan and Ferrari (2021), provides an exact corre spondence (eq. 4.3 and 4.4 ) between tracer observations (the left-hand-side) and the tracer-weighted

\footnotetext{
${ }_{1}^{1}$ Ledwell et al. (2000) interprets it in this way too, but the theoretical scaffolding to contextualize it in the global abyssal circulation was not yet mature (except perhaps in the prescient minds of Munk 1966 and McDougall 1989).
} 
in-situ mixing rates (the right-hand-side). For the BBTRE, Ledwell (personal communication) made a crude first attempt at evaluating these terms, based on the mixing rates produced by a 1D model inversion and objective maps of the tracer and hydrographic observations. This exercise yielded estimates of the LHS and RHS terms that were remarkably similar- within just a few percent of each other (Figure 4-6 d) - suggesting that the two methods are at least generally consistent. When diapycnal stretching effects are large, however, as is expected for the near-bottom BLT release, I expect the tracer-weighted buoyancy moments method to allow more meaningful comparisons of in-situ turbulence estimates against tracer observations, since these diapycnal stretching effects are not easily included in 1D model formulations.

\subsection{Tidal effects on abyssal mixing layers}

To considerably simplify the problem (see Section 1.2), I have omitted ocean tides from all of my solutions and instead opted to parameterize their driving of abyssal turbulence through an imposed bottom-enhanced turbulent diffusivity, $\kappa_{T}$. Thus, I consider my solutions as having filtered out internal waves and as representing averages over spatial- and time-scales longer than those associated with breaking internal waves and other non-hydrostatic processes (e.g. hydraulic jumps). It is certainly possible that my simulations have not accurately represented the net effects of tides on abyssal mixing layers, for example due to: shear dispersion, tidal rectification effects, or turbulent fluxes that are poorly captured by my imposed bottom-enhanced mixing rates (e.g. because they are strongly anisotropic). For example, direct measurements of turbulent fluxes (enabled by remarkable technological advances) in the deep ocean internal tide "swash zone" reveal large cross-slope (nearly horizontal) turbulent fluxes that raise doubts about the conventional assumption of shear turbulence characterized by a bulk mixing coefficient of $\Gamma=0.2$ (Polzin et al., 2021). It is yet unclear what implications these observations have for the paradigm of a BBL-controlled abyssal circulation, but more comprehensive observations of deep BBLs will certainly help to shed light on the situation.

One approach to circumvent the ignorance required by large-scale modelling is to zoom so far in on these processes that they can be directly solved (i.e. accurate direct numerical solution of the full Navier-Stokes equations; see Orszag 1970), as in Kaiser (2020)'s thesis, wherein he uses direct numerical simulations to resolve tidally-driven bottom boundary layer turbulence all the way down to the Kolmogorov scale of molecular dissipation. He finds that the laminar solution becomes gravitationally unstable at one phase of the tide, resulting in asymmetric non-linearities that modify 
the time-mean near-boundary stratification and the up-slope flow relative to the classical laminar Phillips-Wunsch solution (Wunsch, 1970, Phillips, 1970). The trade-off of this setup, however, is that he is restricted to processes up to only a few meters above smoothly sloping seafloor, which contribute little turbulent dissipation and mixing compared to bottom-enhanced observations. Gayen and Sarkar (2011) simulate an analogous problem on the larger scale of the $\mathcal{O}(50 \mathrm{~m})$ bottom boundary layer using (non-rotating) Large Eddy Simulations (LES) for the specific case of critical reflection of an incident internal tide beam. It would be instructive to run similar simulations over sloping rough topography, as Lee et al. (2020) do for the rough stratified Ekman layer without a mean slope (in various parameter regimes relevant to the abyssal ocean).

At even larger scales, the entire life-cycle of the internal wave field can be simulated (again using an LES closure to arrest the turbulent cascade to unresolved small scales): conversion of the barotropic tide into internal tides at rough topography, internal wave propagation, wave-wave interactions, reflection, and breaking (Nikurashin and Legg, 2011; Yi et al., 2017, Jalali and Sarkar, 2017). All of these past studies, however, are inherently transient exercises since there is no forcing that restores the stratification; tidally-driven turbulence homogenizes the near-bottom layers until eventually the stratification becomes too weak to support propagating internal waves, i.e. $N<\omega$. (Transiently, however, internal waves are still generated from the interface at the top of the growing bottom mixed layer). In the present context of mixing-driven bottom boundary layer control on the abyssal circulation, we are more interested in the steady-state dynamics: What maintains the nearbottom stratification that allows internal tides to be generated in the first place (and subsequently drive efficient mixing)? What is the steady-state contribution of these processes to the abyssal overturning circulation?

The two flavors of simulations described above can be combined: Large Eddy Simulations of stratified flow on a slope, forced by an oscillating barotropic tide and where a mean cross-slope flow maintains the stratification of the bottom boundary layer (possibly supplemented by the other restratification processes described in Chapter 3) and allows the evolution towards a non-trivial statistical equilibrium solution. However, such Large Eddy Simulations would depend crucially on the adequacy of sub-grid scale closures for stratified near-boundary flows. I have recently prototyped a hydrostatic implementation of this model setup in the MIT General Circulation Model (Marshall et al. 1997) following the configurations of Chapter 3 and the set-up of Nikurashin and Legg (2011) (using the Klymak and Legg 2010 parameterization to parameterize wave breaking); a nonhydrostatic implementation would probably be preferred, but it may be worth instead implementing 
it in a more efficient and modern non-hydrostatic ocean model, such as SOMAR-LES Chalamalla et al., 2017) or Oceananigans.jl (Ramadhan et al., 2020).

\subsection{Adjustment to non-local interior flows}

Throughout the thesis, I focus on sloping BBL flows forced locally by small-scale turbulent mixing. In addition to generating a buoyancy-driven up-slope flow (the "secondary" circulation), mixing near the boundary causes a horizontal buoyancy gradient which "spins-up" an along-slope thermal wind (the "primary" circulation) (Thorpe, 1987; MacCready and Rhines, 1991). If the naming of these circulations seems backward, it is because their origin traces back to the classical problem of the "spin-down" of a geostrophic current (the "primary" circulation) by frictional Ekman layer flows (the "secondary" circulation). Indeed, a number of other oceanic processes besides local mixing can generate strong along-slope flows, perhaps the most important being boundary current:2 driven by non-local wind (Stommel, 1948) or mixing (Stommel, 1958). Here, I briefly review these Ekman adjustment dynamics and discuss their potential relevance for abyssal watermass transformations.

Let us consider the adjustment of a generic geostrophic flow to friction along a sloping no-slip boundary. As in the classical flat-bottom Ekman layer problem (see Pedlosky, 1987), this externallyimposed along-slope flow sets up a bottom Ekman transport (up- or down-slope, depending on the direction of the along-slope flow) on a fast timescale $f^{-1}$. By continuity, this frictional close-slope flow is returned in the interior, where it is turned into the along-slope direction by the Coriolis force and thus opposes the along-slope that drives it; as a result of this feedback process, the alongslope flow "spins down" on a timescale $\tau_{S}=f^{-1} \mathrm{Ek}^{-1 / 2}$, where Ek $\stackrel{\text { def }}{=} \frac{\nu}{f H^{2}}$ is the Ekman number (Peterson and Callies, in prep). Additionally, however, the cross-slope Ekman transport causes buoyancy surfaces to bend near the boundary, setting up an opposing buoyancy force on a timescale $\tau_{A} \stackrel{\text { def }}{\equiv}(S f)^{-1}$ where $S \stackrel{\text { def }}{=} N^{2} \tan ^{2} \theta / f^{2}$ is the slope Burger number for a fluid with stratification $N^{2}$ and topographic slope angle $\theta$. If $\tau_{A} \ll \tau_{S}$, then this opposing buoyancy force shuts down the cross-slope Ekman transport, dramatically reducing the bottom stress on the along-slope flow and thus "arresting" its spin-down. (MacCready and Rhines, 1991; Garrett et al., 1993). As a result of this buoyancy arrest process, sloping boundaries are more "slippery" than flat-bottom boundaries and are able to sustain stronger along-slope currents (Durrieu De Madron and Weatherly 1994 ,

\footnotetext{
${ }^{2}$ These are generally found on western boundaries due to vorticity constraints and Rossby wave dynamics (Ped losky, 1965, Kawase, 1987), but can be sustained by a combination of topographic vortex stretching and lateral eddy buoyancy fluxes (Yang et al. 2020, 2021).
} 
see also laboratory experiments by MacCready and Rhines 1991). In the canonical 1D sloping BBL initial value problem, the Ekman adjustment solution spins down to the same steady state as the solution that is spun-up from rest by mixing (Garrett et al., 1993). However, to the extent that external forces maintain an along-slope flow or that cross-slope pressure gradients modify the cross-slope transport, this canonical 1D model may not adequately characterize the near-boundary stratification and the resulting watermass transformations (Garabato et al. 2019, Spingys et al. 2021 Peterson and Callies, in prep.).

To further complicate matters, the linear sloping Ekman layer solutions discussed above are subject to various flow instabilities which substantially modify the canonical solution. The most basic of these processes is gravitational instability, which is especially common in the case of a downwelling-favorable along-slope flow that drives a down-slope transport of buoyant waters, re sulting in convective mixing that progressively thickens the bottom boundary layer (Garrett et al. 1993 Ruan et al. 2019). Whether the linear solution is originally gravitationally stable or only becomes so after a period of convective mixing (Ruan et al. 2019), it may yet exhibit negative Er tel potential vorticity that renders it susceptible to centrifugal, symmetric, and mixed instabilities Haine and Marshall, 1998; Garabato et al., 2019; Wenegrat et al., 2018; Wenegrat and Thomas, 2020). The effect of all of these instabilities is to restratify the bottom boundary layer and pro mote exchange with these interior, enhancing watermass transformations in the BBL. To the extent that these instabilities drive significant small scale turbulence (relative to other processes, such as boundary layer and internal wave shear turbulence), they may also modify the efficiency with which this turbulent production results in irreversible mixing (Garabato et al., 2019, Spingys et al., 2021). Finally, we note that in reality, along-slope flows have finite width and thus drive locally-closed sec ondary circulations (Benthuysen, 2010), which can evolve asymmetrically due to non-linear effects (Benthuysen and Thomas, 2013).

\subsection{Towards predictive models of boundary-interior exchange}

The simulation results in Chapters 2 and 3 provide some of the first quantitative evidence for the often-repeated speculation that boundary-interior exchange flows driven by along-slope heterogene ity (in mixing rates, the background stratification, or topographic slopes) contribute significantly to the abyssal overturning circulation (Armi, 1978; Phillips et al., 1986; McDougall, 1989, Garrett, 1990; Salmun et al., 1991; Kunze et al., 2012; Dell and Pratt, 2015). It is useful to separate these 
exchange flows into two categories: 1) basin-scale exchanges between the sloping mixing layers and the interior and 2) local slope-normal exchange flows within sloping mixing layers, e.g. eddy fluxes between the BBL and the SML.

Chapter 2 explores the first case, where an increasing slope at the base of the mid-ocean ridge causes buoyancy flux convergence in the BBL and a decreasing slope at the ridge crest causes buoyancy flux divergence; at equilibrium, however, vertical variations in the interior stratification drive leading-order convergences at all depths (see also Spingys et al., 2021; Holmes et al., 2018). The asymptotic model developed by Salmun et al. (1991) ${ }^{3}$ provides first-order corrections to the 1D boundary layer solutions due to pycnocline-induced convergences along the slope and frame the effects of these exchange flows in terms of an effective interior diffusivity. However, their poorlyjustified choices of non-dimensional parameters and the complexity of the resulting first-order system make the overall approach exceedingly difficult to follow and yield unlikely scaling laws (e.g. interior diffusivity scales like the inverse cube of the turbulent layer's diffusivity, $\kappa_{\mathrm{I}} \propto \kappa^{-3}$ ). In Appendix A I have made some first attempts at setting up a simpler problem (e.g. using Rayleigh drag instead of momentum diffusion and some convenient assumptions about the parameter regime that simplify the non-dimensionalization), but have not yet searched for analytical or numerical solutions. Given the importance of this boundary-interior coupling in the emerging conception of the abyssal circulation, this decades-old problem should be revisited, perhaps with a bit more guidance from heuristic or scaling arguments.

Chapter 3 explores the second case, where cross-slope (and along-slope) variations in the topo graphic slope drive slope-normal exchanges within the BBL and SML, and between the two. Dell (2013) describes these exchange flows extensively using various two- and three-dimensional simu lations; she provides some useful scaling laws that predict properties of the exchange flows and transitions from linear to non-linear to overflow regimes, but does not derive any general analytical solutions. In Dell and Pratt (2015), their simulation results for mixing-driven boundary layer flow over small-amplitude cross-slope corrugations are strongly suggestive of the classical (non-sloping) lee wave problem 4 , suggesting an asymptotic approach may be used to solve for: the smoothlysloping boundary layer solution, at leading order; the corrugation-induced exchange flows, at first

\footnotetext{
${ }^{3}$ The asymptotic models of Woods $(1991)$ and Imberger and Ivey (1993) are also noteworthy, but the problems they set up are not as relevant to abyssal mixing layers, in my opinion.

${ }^{4}$ The major complication seems to be that the leading-order boundary flows vary rapidly in the vertical (on the $\mathcal{O}(10 \mathrm{~m})$ scale of even quite small topography) and conventionally satisfy a no-slip condition at the seafloor, so it is not clear what "bottom" velocity to use to generate the lee waves (Legg, 2021). Polzin (2009)'s bottom-enhanced mixing recipe suffers from a similar problem since the stratification of the bottom grid cell is a key input which likely also depends strongly on the model's vertical resolution.
} 
order; and perhaps insightful wave-mean or wave-wave interactions, at higher order (e.g., following Nikurashin and Ferrari, 2009). In Chapter 3, I find topographically-induced standing eddies (devia tions from the slope-normal mean) contribute somewhat to the buoyancy budget of a fracture zone canyon, although the nature and impact of these topographic eddies remain unclear.

In Chapter 2, I test Munk 1966)'s classic vertical advection-diffusion model (re-interpreted as a basin-scale average as in Munk and Wunsch 1998) by comparing the left- and right-hand-side terms of their equations (Figure 2-13). The vertical turbulent buoyancy flux divergence provides a poor prediction of the upwelling transport in the basin. This has implications for the fidelity of zonallyintegrated theories of the meridional overturning circulation (Nikurashin et al., 2012, e.g.), which typically assume a Munk-like basin in the abyssal ocean (outside of the Southern Ocean). Reframing this vertical balance in buoyancy coordinates and accounting for hyposometric effects (e.g. Jansen and Nadeau, 2019) would likely improve the solution, but the decomposition in Figure 2-14 suggests that the omitted spatial correlation terms between the prescribed mixing $\kappa$ and the prognostic stratification $b_{z}$ lead to errors of up to $100 \%$ (the impact of these correlations is a bit muted in the supporting simulation results of Spingys et al. 2021, but are still as large as 50\%). It may be possible to develop an analytical (or idealized numerical model) for the abyssal overturning circulation based on the coupled evolution of bottom boundary layers and the interior, which would not suffer from these assumptions about horizontal homogeneity; alternatively, better understanding of these coupling processes could allow a simpler parameterization, such as a parameterized enhancement of the horizontally-averaged diffusivity that accounts for missing buoyancy fluxes in abyssal mixing layers (as in Salmun et al., 1991).

\subsection{Sub-gridscale parameterization in general circulation models}

The width of my simulation domain in Chapter 3 is comparable to the nominal width of a modern general circulation model's (GCMs) oceanic grid cell; thus, the processes described in Chapter 3 occur below the gridscale and thus can only be included in GCM solutions through parameteriza tion. The hierarchy of models approach in Chapter 3 revealed three key subgrid-scale processes that control the near bottom stratification (and thus, watermass transformations): finite-amplitude sub mesoscale baroclinic eddies, blocking of cross-canyon thermal wind by narrowly separated sidewalls, and blocking of up-slope restratification of deep depressions by topographic sills.

The restratification effect of submesoscale bottom mixing layer eddies, which we might expect 
to dominate over hilly ridge flanks, could conceivably be parameterized as an eddy overturning streamfunction, as has been successfully implemented for restratification by surface mixed layer eddies in GCMs (Boccaletti et al., 2007; Fox-Kemper et al., 2008, 2011). Reassuringly, a bottom mixing layers parameterization for submesoscale symmetric instability (intended for overflows) has also recently been implemented in a GCM (Yankovsky and Legg, 2019, Yankovsky et al., 2020).

To some extent, the effects of larger-scale topographic features, such as canyons and their block ing sills, can be resolved by simply increasing the resolution a bit; for example, high-resolution simulations with nominal resolutions of about $1 / 10^{\circ}$ or $10 \mathrm{~km}$, which likely marginally resolve most fracture zone canyons of widths of $\mathcal{O}(20 \mathrm{~km})$, are now increasingly feasible. If canyons are not explicitly resolved, but are thought to be sufficiently important that they should be included anyways, they can be "dug out" by altering the bathymetric input files; this is already a widely-used practice for including channels such as the Denmark Strait, the Faroe-Bank Channel, and the Strait of Gibraltar that have leading order effects on global ocean circulation and tracer distributions. Finally, if the dynamical effects of the topography on the flow are sufficiently well understood, they can be parameterized by numerical "piping", as in Polzin (2009)'s Abyssal Recipe for upwelling by mixing above unresolved abyssal hills or for narrow hydraulically-controlled channels and their associated downstream dense overflows (Danabasoglu et al., 2010; Legg et al., 2009).

\subsection{The role of abyssal mixing layers in climate}

Little is known about the role of abyssal mixing layers in climate. In part, this is because the emerging paradigm of boundary layer control of the abyssal circulation is relatively new and has not yet been analyzed in the context of a realistic global ocean or climate model. To the extent that abyssal mixing layer dynamics are resolved (or parameterized) in climate models, I expect them to have an impact on climate through their modulation of the large-scale abyssal circulation. In deed, we may get some hints from the substantial literature on the role of diapycnal mixing and the bottom-enhancement of diapycnal mixing on the abyssal circulation and various downstream climate impacts. Saenko and Merryfield (2005) show that, relative to the then-standard practice of assum ing a uniform (or piece-wise uniform, as in Bryan and Lewis 1979) diapycnal diffusivity, prescribing a realistically bottom-enhanced diffusivity improves the fidelity of the solution by strengthening the abyssal stratification and circulation in the Southern and Pacific Oceans. Friedrich et al. (2011) extend the impact of these oceanic changes by considering an ocean-atmosphere-biogeochemistry 
model, and show that the bottom-enhancement of mixing additionally results in significant changes to surface air temperatures, atmospheric circulation patterns, and global biogeochemical cycling. Melet et al. (2013) show that not only do the magnitude and horizontal structure of the parameter ized mixing matter, but its vertical distribution too (using Polzin 2009 s dynamical parameterization of vertical internal-tide propagation, interaction, and breaking).

To date, most studies of the impact of diapycnal mixing parameterizations on global climate have focused on the storage of atmospherically-sourced tracers (or out-gassing of stored tracers). Our emphasis on the role of bottom mixing layers, however, suggests we should turn to climatically-active tracers sourced from the bottom boundary. The climatically important hydrothermal fluxes of iron (Tagliabue et al., 2010), for example, are likely to be strongly modulated by the mixing and flows described in this thesis; imagine for a second that the tracers in Chapter 3 were hydrothermal plumes rich in nutrients (such as iron) that, although buoyant upon release, have settled at a neutrallybuoyant level near the boundary. Indeed, hydrographic observations of a particular hydrothermal iron plume show its spreading across from its localized release near the crest of a mid-ocean ridge (Resing et al., 2015). Modern ocean biogeochemistry models still struggle to reproduce dissolved iron distributions (Tagliabue et al., 2016), and only models that include hydrothermal iron sources have any resemblance to the observations in the abyss (but even those correlations are quite poor).

Mitigating human-induced climate change requires a broad portfolio of climate solutions. At least two important speculative climate solutions involve abyssal mixing layers: 1) enhancing the natural export of atmospheric carbon into the deep ocean (known as Ocean-based Carbon Dioxide Removal) and 2) the extraction of minerals required for renewable energy technologies (e.g. cobalt) from abundant reserves along the abyssal seafloor (known as Deep Sea Mining). The effectiveness of OCDR depends on the timescale over which the sequestered carbon (in the form of remineralized dissolved oceanic carbon in the BBL) remains isolated from the atmosphere (Siegel et al., 2021). Improved understanding of abyssal mixing layer dynamics, and their coupling to the large scale circulation, would improve estimates of the permanence of OCDR and allow for the identification of optimal sequestration sites. An appeal of DSM is that any environmental impacts would be far removed from humans and the familiar ecosystems that surround us, especially as compared to terrestrial mining of the same resources; but how remote are these impacts really? A major environmental impact of DSM is the dispersion of sediment plumes (both in the bottom mixing layers as a direct result of mining operations and in the interior as a result of unwanted discharged sediment) which potentially smother filter feeders and carry toxic metals (Peacock and Alford, 2018). 
Important inputs for environmental impact assessments, such as the seafloor area affected by these

plumes and the Lagrangian connectivity of benthic ecosystems, are poorly known but expected to be controlled by the dynamics of abyssal mixing layers.

\subsection{Towards an abyssal cookbook}

After six decades of progress on the problem of the mixing-driven abyssal overturning, it seems the recipes are getting delicious enough to soon publish a cookbook 5

${ }^{5}$ See the upcoming book Ocean Mixing: Drivers, Mechanisms and Impacts edited by Meredith and Garabato (2021) for a modern account, for example. 


\section{Appendix A}

\section{Asymptotics of pycnocline-induced boundary layer exchange flows}

\section{A.1 The planetary geostrophic equations in slope coordinates}

Motivated by the impressive but convoluted asymptotic approach of Salmun et al. (1991), we aim to derive a more minimal and more interpretable model of the coupling between abyssal mixing layers and the interior due to vertical variations in the interior stratification. Our set-up of the problem follows that of (Salmun et al. 1991), but we consider a specific parameter regime appropriate for abyssal mixing layers.

Following Chapter 2, the steady planetary geostrophic equations for a stratified, rotating fluid with friction provided by a linear (Rayleigh) drag are:

$$
\begin{array}{rlr}
-f \tilde{v} & =-p_{\tilde{x}}-r \tilde{u} & \text { zonal momentum } \\
f \tilde{u} & =-p_{\tilde{y}}-r \tilde{v} & \text { meridional momentum } \\
0 & =-p_{\tilde{z}}+b & \text { hydrostatic balance } \\
\tilde{u} b_{\tilde{x}}+\tilde{w} b_{\tilde{z}} & =\partial_{\tilde{x}}\left(\kappa b_{\tilde{x}}\right)+\partial_{\tilde{z}}\left(\kappa b_{\tilde{z}}\right) & \text { buoyancy, }
\end{array}
$$

where we set up the problem such that there are no variations in the meridional direction, i.e. $p_{\tilde{y}}=b_{\tilde{y}}=0$. Rotating into the slope reference frame $(x, y, z)=(\tilde{x} \cos \theta+\tilde{z} \sin \theta, \tilde{y}, \tilde{z} \cos \theta-\tilde{x} \sin \theta)$ 
where $(\tilde{x}, \tilde{y}, \tilde{z})=(x \cos \theta-z \sin \theta, y, z \cos \theta+x \sin \theta)$, we have

$$
\begin{array}{rlrl}
-f v \cos \theta & =-p_{x}+b \sin \theta-r \cos \theta(u \cos \theta-w \sin \theta) & & \text { cross-slope momentum } \\
f(u \cos \theta-w \sin \theta) & =-r v & & \text { along-slope momentum } \\
f v \sin \theta & =-p_{z}+b \cos \theta+r \sin \theta(u \cos \theta-w \sin \theta) & & \text { slope-normal momentum } \\
u b_{x}+w b_{z} & =\partial_{x}\left(\kappa b_{x}\right)+\partial_{z}\left(\kappa b_{z}\right) & \text { buoyancy. }
\end{array}
$$

Combining the cross-slope and slope-normal momentum equations into an equation for the slope-normal vorticity, we have

$$
\begin{aligned}
-f\left(v_{x} \sin \theta+v_{z} \cos \theta\right)= & b_{z} \sin \theta-b_{x} \cos \theta-r\left[\cos \theta\left(u_{z} \cos \theta-w_{z} \sin \theta\right)\right. \\
& \left.+\sin \theta\left(u_{x} \cos \theta-w_{x} \sin \theta\right)\right] \\
f(u \cos \theta-w \sin \theta)= & -r v \\
u b_{x}+w b_{z}= & \partial_{x}\left(\kappa b_{x}\right)+\partial_{z}\left(\kappa b_{z}\right)
\end{aligned}
$$$$
\text { along-slope vorticity }
$$

along-slope momentum

\section{A.2 The creeping flow equations in slope coordinates}

Since the coupling process is inherently buoyancy-driven, we begin with the simpler non-rotating case. For a non-rotating flow $(f=0)$, these equations simplify to

$$
\begin{aligned}
& b_{z} \sin \theta-b_{x} \cos \theta=r\left[\cos \theta\left(u_{z} \cos \theta-w_{z} \sin \theta\right)+\sin \theta\left(u_{x} \cos \theta-w_{x} \sin \theta\right)\right] \quad \text { along-slope vorticity } \\
& 0=-r v \\
& \text { along-slope momentum } \\
& u b_{x}+w b_{z}=\partial_{x}\left(\kappa b_{x}\right)+\partial_{z}\left(\kappa b_{z}\right)
\end{aligned}
$$


where the along-slope momentum equation implies that there is no along-slope velocity, $v=0$.

We now decompose the buoyancy field into a far-field component and a perturbation,

$$
b(x, z)=\bar{B}(\tilde{z})+b^{\prime}(x, z)
$$

where $\frac{\mathrm{d} \bar{B}}{\mathrm{~d} \tilde{z}}=N^{2}$. We define a streamfunction by $u=\psi_{z}$ and $w=-\psi_{x}$. In terms of the buoyancy perturbations and the streamfunction, the equations become

$$
\begin{aligned}
& b_{z}^{\prime} \sin \theta-b_{x}^{\prime} \cos \theta=r\left[\psi_{z z} \cos ^{2} \theta+2 \psi_{z x} \sin \theta \cos \theta+\psi_{x x} \sin ^{2} \theta\right] \\
& \psi_{z} b_{x}^{\prime}-\psi_{x} b_{z}^{\prime}+\psi_{z} N^{2} \sin \theta-\psi_{x} N^{2} \cos \theta=\partial_{\tilde{z}}\left(\kappa N^{2}\right)+\partial_{x}\left(\kappa b_{x}^{\prime}\right)+\partial_{z}\left(\kappa b_{z}^{\prime}\right)
\end{aligned}
$$

where we note that $\bar{B}_{z} \sin \theta-\bar{B}_{x} \cos \theta=N^{2} \cos \theta \sin \theta-N^{2} \sin \theta \cos \theta=0$. Notably, this differs from Imberger and Ivey (1993)'s model, since their background state only varies in the cross-slope direction $\bar{B} \equiv \bar{B}(x)$ and is apparently unbalanced; as a result, the background buoyancy gradient $\bar{B}_{x}$ enter their system at leading order.

We non-dimensionalize by $x=\hat{x} L, z=\hat{z} H$, and $\tilde{z}=\hat{z} h$, where $H$ is the thickness of the turbulent bottom boundary layer, $h$ is the height over which the stratification varies, and $L=h / \sin \theta$ is the projection of $h$ onto the cross-slope direction. We write $N^{2}=N_{0}^{2} \mathcal{G}(\tilde{z})$, where $N_{0}^{2}$ is a reference stratification and $\mathcal{G}(\tilde{z})$ is a non-dimensional function of $\mathcal{O}(1)$, and $b^{\prime}=\hat{b} N_{0}^{2} H$. The streamfunction is scaled as $\psi=\hat{\psi} Q$ where $Q$ is determined by asserting that the leading order buoyancy balance should be the Phillips (1970) balance between cross-slope advection of background buoyancy and the slope-normal diffusion of buoyancy,

$$
\psi_{z} N^{2} \sin \theta=\partial_{z}\left(\kappa b_{z}\right)
$$

which gives a scaling of $Q=\kappa \sin ^{-1} \theta$. Since we will only consider small angles $\theta \ll 1$, taking $\sin \theta \approx \theta$ and $\cos \theta \approx 1$ greatly simplifies our equations. For simplicitly, we assume the diffusivity $\kappa$ is constant within the turbulent layer of thickness $H$, and vanishes in the interior. 
In the turbulent layer, the non-dimensional equations are then given by

$$
\begin{gathered}
\hat{b}_{\hat{z}}^{\prime}-\left(\frac{H \theta}{h}\right) \hat{b}_{\hat{x}}^{\prime}=\left(\frac{r \kappa}{N_{0}^{2} H^{2}}\right)\left[\hat{\psi}_{\hat{z} \hat{z}}+2\left(\frac{H \theta^{2}}{h}\right) \hat{\psi}_{\hat{x} \hat{z}}+\left(\frac{H^{2} \theta^{6}}{h^{2}}\right) \hat{\psi}_{\hat{x} \hat{x}}\right] \\
\hat{\psi}_{\hat{z}} \mathcal{G}(\tilde{z})+\left(\frac{H}{h}\right)\left[\hat{\psi}_{\hat{z}} \hat{b}_{\hat{x}}^{\prime}-\hat{\psi}_{\hat{x}} \hat{b}_{\hat{z}}^{\prime}-\hat{\psi}_{\hat{x}} \mathcal{G}(\tilde{z})\right]=\hat{b}_{\hat{z} \hat{z}}^{\prime}+\mathcal{G}_{\hat{z}}+\left(\frac{H \theta^{2}}{h}\right) \hat{\mathcal{G}}_{\hat{x}}+\left(\frac{H^{2} \theta^{2}}{h^{2}}\right) \hat{b}_{\hat{x} \hat{x}}^{\prime},
\end{gathered}
$$

where we recognize $R a \equiv \frac{N_{0}^{2} H^{2}}{r \kappa}$ as the Rayleigh number. We now consider the limit $\epsilon \equiv H / h \ll 1$, where $\epsilon$ is the aspect ratio of bottom boundary layer flow; for a boundary layer thickness of $H=$ $100 \mathrm{~m}$ and a vertical stratification scale $h=1000 \mathrm{~m}$, the aspect ratio is indeed small, $\epsilon \approx 0.1 \ll 1$. For $R a=\mathcal{O}(1)$, we retrieve the $1 \mathrm{D}$ boundary layer model from Chapter 1 (eq. 2.21-2.23) at leading order (where we have set $f=0$ ):

$$
\begin{gathered}
\hat{b}_{\hat{z}}^{\prime} \simeq \hat{\psi}_{\hat{z} \hat{z}} \\
\hat{\psi}_{\hat{z}} \mathcal{G} \simeq \hat{b}_{\hat{z} \hat{z}}^{\prime}
\end{gathered}
$$

For simplicity, we set $R a \equiv 1$. As long as $\theta \leq \epsilon$, the leading order and first order equations are unchanged so without loss of generality we consider $\theta=\epsilon^{3} \approx 10^{-3}$ (appropriate for the large-scale mid-ocean ridges considered in this thesis). The equations can now be expressed in terms of a single non-dimensional parameter, the aspect ratio $\epsilon$

$$
\begin{gathered}
\hat{b}_{\hat{z}}^{\prime}-\epsilon^{4} \hat{b}_{\hat{x}}^{\prime}=\hat{\psi}_{\hat{z} \hat{z}}+2 \epsilon^{7} \hat{\psi}_{\hat{x} \hat{z}}+\epsilon^{20} \hat{\psi}_{\hat{x} \hat{x}} \\
\hat{\psi}_{\hat{z}} \mathcal{G}(\tilde{z})+\epsilon\left[\hat{\psi}_{\hat{z}} \hat{b}_{\hat{x}}^{\prime}-\hat{\psi}_{\hat{x}} \hat{b}_{\hat{z}}^{\prime}-\hat{\psi}_{\hat{x}} \mathcal{G}(\tilde{z})\right]=\hat{b}_{\hat{z} \hat{z}}^{\prime}+\mathcal{G}_{\hat{z}}+\epsilon^{7} \mathcal{G}_{\hat{x}}+\epsilon^{8} \hat{b}_{\hat{x} \hat{x}}^{\prime}
\end{gathered}
$$

Finally, we express the $\mathcal{O}(1)$ structure function for stratification as $\mathcal{G}(\tilde{z})=1+\epsilon g(\tilde{z})$, where $g(\tilde{z})$ is an $\mathcal{O}(1)$ structure function for the small perturbation away from a constant stratification. Expanding, we have

$$
\begin{gathered}
\hat{b}_{\hat{z}}^{\prime}-\epsilon^{4} \hat{b}_{\hat{x}}^{\prime}=\hat{\psi}_{\hat{z} \hat{z}}+2 \epsilon^{7} \hat{\psi}_{\hat{x} \hat{z}}+\epsilon^{20} \hat{\psi}_{\hat{x} \hat{x}} \\
\hat{\psi}_{\hat{z}}+\epsilon\left[\hat{\psi}_{\hat{z}} g(\tilde{z})+\hat{\psi}_{\hat{z}} \hat{b}_{\hat{x}}^{\prime}-\hat{\psi}_{\hat{x}} \hat{b}_{\hat{z}}^{\prime}-\hat{\psi}_{\hat{x}}\right]-\epsilon^{2} \hat{\psi}_{\hat{x}} g(\tilde{z})=\hat{b}_{\hat{z} \hat{z}}^{\prime}+\epsilon g_{\hat{z}}+\epsilon^{8} g_{\hat{x}}+\epsilon^{8} \hat{b}_{\hat{x} \hat{x}}^{\prime}
\end{gathered}
$$




\section{A.2.1 Boundary conditions}

\section{Bottom boundary condition}

The bottom boundary conditions are the conditions of no normal flow at the sloping boundary and no buoyancy flux across the sloping boundary,

$$
\begin{array}{lr}
\psi_{x}=0 & \text { at } z=0 \\
b_{z}^{\prime}+N_{0}^{2} \cos \theta=0 & \text { at } z=0 .
\end{array}
$$

Or, non-dimensionally,

$$
\begin{array}{lr}
\hat{\psi}_{\hat{x}}=0 & \text { at } \hat{z}=0 \\
\hat{b}_{\hat{z}}^{\prime}+1+\epsilon g(\tilde{z})=0 & \text { at } \hat{z}=0 .
\end{array}
$$

\section{Matching conditions with the quiescent interior}

To close the system of equations, we need two more boundary conditions. We find two boundary conditions at the top of the bottom boundary layer by matching the buoyancy and vorticity balances on the inside and outside of the layer.

Let $\delta \ll H$ be some small distance. We assert that the slope-normal velocity $w$ must be continuous in the slope normal direciton $z$ (otherwise $u$ is discontinuous in $x$ for all $x$ along the top of the boundary layer). However, we allow a jump in the buoyancy $b$ across the top of the boundary layer. Integrating the buoyancy equation from $z=H-\delta$ to $z=H+\delta$, we have

$$
\int_{H-\delta}^{H+\delta} \partial_{x}(u b)+\partial_{z}(w b)-\partial_{x}\left(\kappa b_{x}\right)-\partial_{z}\left(\kappa b_{z}\right) z=0
$$

As $\delta \rightarrow 0$, the cross-slope divergence terms are finite values that scale with $2 \delta$ and thus vanish. We are left with

$$
\left[w b-\kappa b_{z}\right]_{H-\delta}^{H+\delta}=0
$$

Since $w$ is continuous across the top of the boundary layer, $w(H+\delta)=w(H-\delta)=w$. Outside of the boundary layer, $\kappa=0, b=\bar{B}$, and $b_{z}=N^{2} \cos \theta$. Inside of the boundary layer, $\kappa=\kappa_{0}$, 
$b=\bar{B}+b^{\prime}$, and $b_{z}=N^{2} \cos \theta+b_{z}^{\prime}$. Thus, we have

$$
0=w b^{\prime}-\kappa\left(b_{z}^{\prime}+N^{2} \cos \theta\right)
$$

as a boundary condition at $z=H$. Integrating the vorticity equation, we have

$$
\int_{H-\delta}^{H+\delta} b_{z} \sin \theta-b_{x} \cos \theta-r\left[\cos \theta\left(u_{z} \cos \theta-w_{z} \sin \theta\right)+\sin \theta\left(u_{x} \cos \theta-w_{x} \sin \theta\right)\right] \mathrm{d} z=0
$$

which becomes

$$
[b \sin \theta-r \cos \theta(u \cos \theta-w \sin \theta)]_{H-\delta}^{H+\delta}=0 .
$$

Outside of the boundary layer, there is no diabatic source of buoyancy so $\tilde{w}=w \cos \theta+u \sin \theta=0$, which we rearrange to give $u=-w \tan ^{-1} \theta$ for $z>H$. Since the background buoyancy and slopenormal velocity are continuous across the top of the boundary, we are left with

$$
r w \cos ^{2} \theta \tan ^{-1} \theta=b^{\prime} \sin \theta-r u \cos \theta \sin \theta
$$

as our second boundary condition at $z=H$.

Note: We require the slope-normal velocity $w$ to be continuous in $z$ across the top of the boundary layer but this need not apply to the cross-slope velocity, $u$, the true horizontal velocity $\tilde{u}$, or the true vertical velocity $\tilde{w}$.

Non-dimensionally, the boundary conditions at the top of the boundary layer become

$$
\begin{array}{lr}
\hat{\psi}_{\hat{z}}-\hat{b}^{\prime}-\epsilon^{7} \hat{\psi}_{\hat{x}}=0 & \text { at } \hat{z}=1 \\
\hat{b}_{\hat{z}}^{\prime}+1+\epsilon g(\tilde{z})-\epsilon \hat{w} \hat{b}^{\prime}=0 & \text { at } \hat{z}=1
\end{array}
$$




\section{A.2.2 Non-dimensional problem}

All together, the non-dimensional problem is given by

$$
\begin{array}{ll}
\hat{b}_{\hat{z}}^{\prime}-\epsilon^{4} \hat{b}_{\hat{x}}^{\prime}=\hat{\psi}_{\hat{z} \hat{z}}+2 \epsilon^{7} \hat{\psi}_{\hat{x} \hat{z}}+\epsilon^{20} \hat{\psi}_{\hat{x} \hat{x}} & \\
\hat{\psi}_{\hat{z}}+\epsilon\left[\hat{\psi}_{\hat{z}} g(\tilde{z})+\hat{\psi}_{\hat{z}} \hat{b}_{\hat{x}}^{\prime}-\hat{\psi}_{\hat{x}} \hat{b}_{\hat{z}}^{\prime}-\hat{\psi}_{\hat{x}}\right]-\epsilon^{2} \hat{\psi}_{\hat{x}} g(\tilde{z})=\hat{b}_{\hat{z} \hat{z}}^{\prime}+\epsilon g_{\hat{z}}+\epsilon^{8} g_{\hat{x}}+\epsilon^{8} \hat{b}_{\hat{x} \hat{x}}^{\prime} . & \\
\hat{\psi}_{\hat{x}}=0 & \text { at } \hat{z}=0 \\
\hat{b}_{\hat{z}}^{\prime}+1+\epsilon g(\tilde{z})=0 & \text { at } \hat{z}=0 . \\
\hat{\psi}_{\hat{z}}-\hat{b}^{\prime}-\epsilon^{7} \hat{\psi}_{\hat{x}}=0 & \\
\hat{b}_{\hat{z}}^{\prime}+1+\epsilon g(\tilde{z})+\epsilon \hat{\psi}_{\hat{x}} \hat{b}^{\prime}=0 & \text { at } \hat{z}=1
\end{array}
$$

\section{A.3 Regular perturbation expansion in aspect ratio $\epsilon$}

We now consider a regular perturbation expansion in the small aspect ratio $\epsilon$ (and, for clarity, drop the hats that denote non-dimensional variables), such that $\psi=\psi_{0}+\epsilon \psi_{1}+\ldots$ and $b^{\prime}=b_{0}^{\prime}+\epsilon b_{1}^{\prime}+\ldots$

\section{A.3.1 $\mathcal{O}(1)$ solution}

We retrieve the $1 \mathrm{D}$ boundary layer equations from Chapter 1 for $f=0$, where only the top boundary condition has been modified

$$
\begin{array}{ll}
b_{0_{z}}^{\prime}=\psi_{0_{z z}} & \\
\psi_{0_{z}}=b_{0_{z z}}^{\prime} & \\
\psi_{0_{x}}=0 & \text { at } z=0 \\
b_{0_{z}}^{\prime}+1=0 & \text { at } z=0 \\
\psi_{0_{z}}-b_{0}^{\prime}=0 & \text { at } z=1 \\
b_{0_{z}}+1=0 & \text { at } z=1
\end{array}
$$


We note that there is no dependence on $x$ in the $\mathcal{O}(1)$ problem and so $b_{0}=b_{0}(z)$ and $\psi_{0}=\psi_{0}(z)$.

\section{A.3.2 $\mathcal{O}(\epsilon)$ solution}

The $\mathcal{O}(\epsilon)$ equations are identical to the $\mathcal{O}(1)$ equations except that the buoyancy equation contains two additional forcing terms corresponding to advection of the perturbed background stratification by the $\mathcal{O}(1)$ flow and turbulent mixing across the perturbed background stratification.

$$
\begin{array}{ll}
b_{1_{z}}^{\prime}=\psi_{1_{z z}} & \\
\psi_{1_{z}}+\psi_{0_{z}} g=b_{1_{z z}}^{\prime}+g_{z} & \\
\psi_{1_{x}}=0 & \text { at } z=0 \\
b_{1_{z}}^{\prime}+g=0 & \text { at } z=0 \\
& \\
\psi_{1_{z}}-b_{1}^{\prime}=0 & \text { at } z=1 \\
b_{1_{z}}^{\prime}+g=0 & \text { at } z=1
\end{array}
$$

Since $g=g(\tilde{z})=g(x, z)$, the $\mathcal{O}(\epsilon)$ solution will depend on $x$ and drives a 'tertiary' exchange flow $\left.w\right|_{z=1}=\left.\psi_{x}\right|_{z=1} \neq 0$ between the turbulent bottom boundary layer and the quiescent interior.

The general solution to the $\mathcal{O}(1)$ problem is a sum of exponentials, with coefficients determined by the boundary conditions. For elementary choices of the perturbed background stratification $g(\tilde{z})$ that are periodic in $x$, it seems plausible that there may be analytical solutions to the $\mathcal{O}(\epsilon)$ problem that provide insight into the coupling between the mixing layers and the interior. However, we expect that the exchange flows produced by the $\mathcal{O}(\epsilon)$ solution will inevitably feedback upon the interior solution on some slow timescale. Coupling the boundary layer model above to a model for the interior stratification (see Section 5 of Salmun et al. 1991 for a simple "filling-box" model) may provide insight into the long-term equilibration of the basin-wide stratification, as described for the idealized general circulation model Chapter 1. 


\section{Appendix B}

\section{Figures}
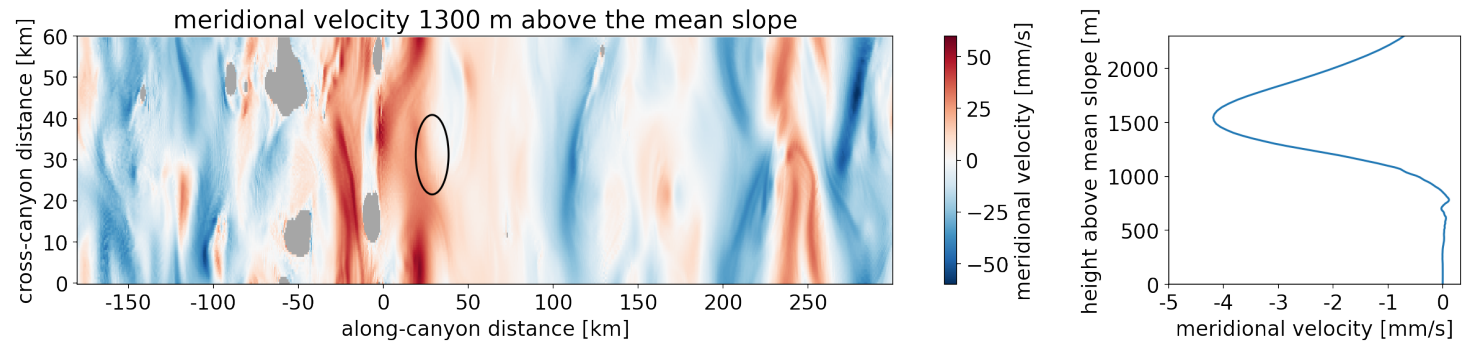

Figure B-1: (a) Cross-canyon meridional velocity $1300 \mathrm{~m}$ above the mean slope, i.e. at the release height of BBTRE tracer. (b) Same as a), but averaged across the whole domain. 

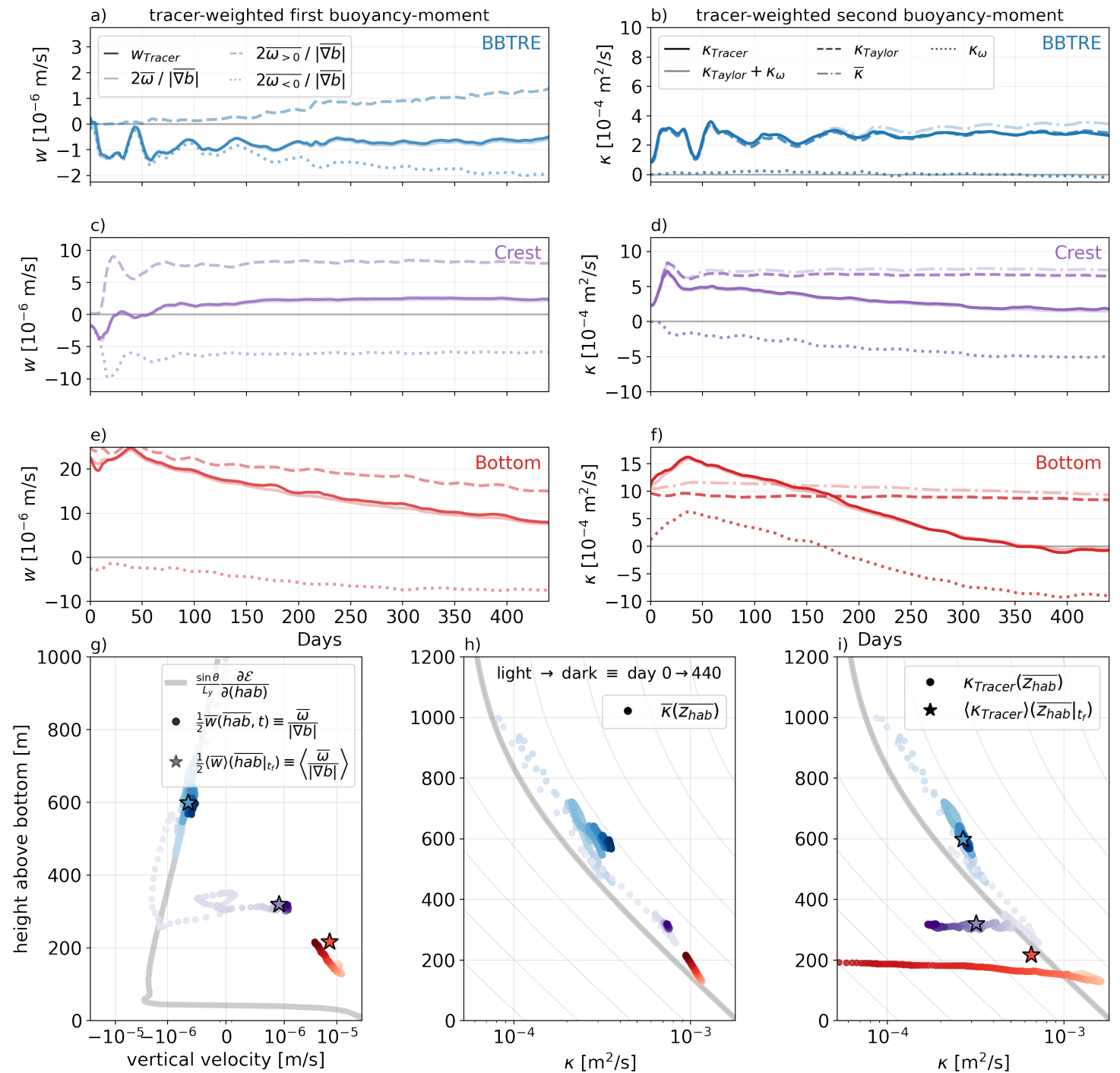

Figure B-2: As in Figure 4-6, but after normalizing (a,c,e) by $\overline{|\nabla T|}$ and (b,d,f) by $\overline{|\nabla T|^{2}}$ to convert to units of velocity and diffusivity, respectively, as in equations 4.3 and 4.4 . 

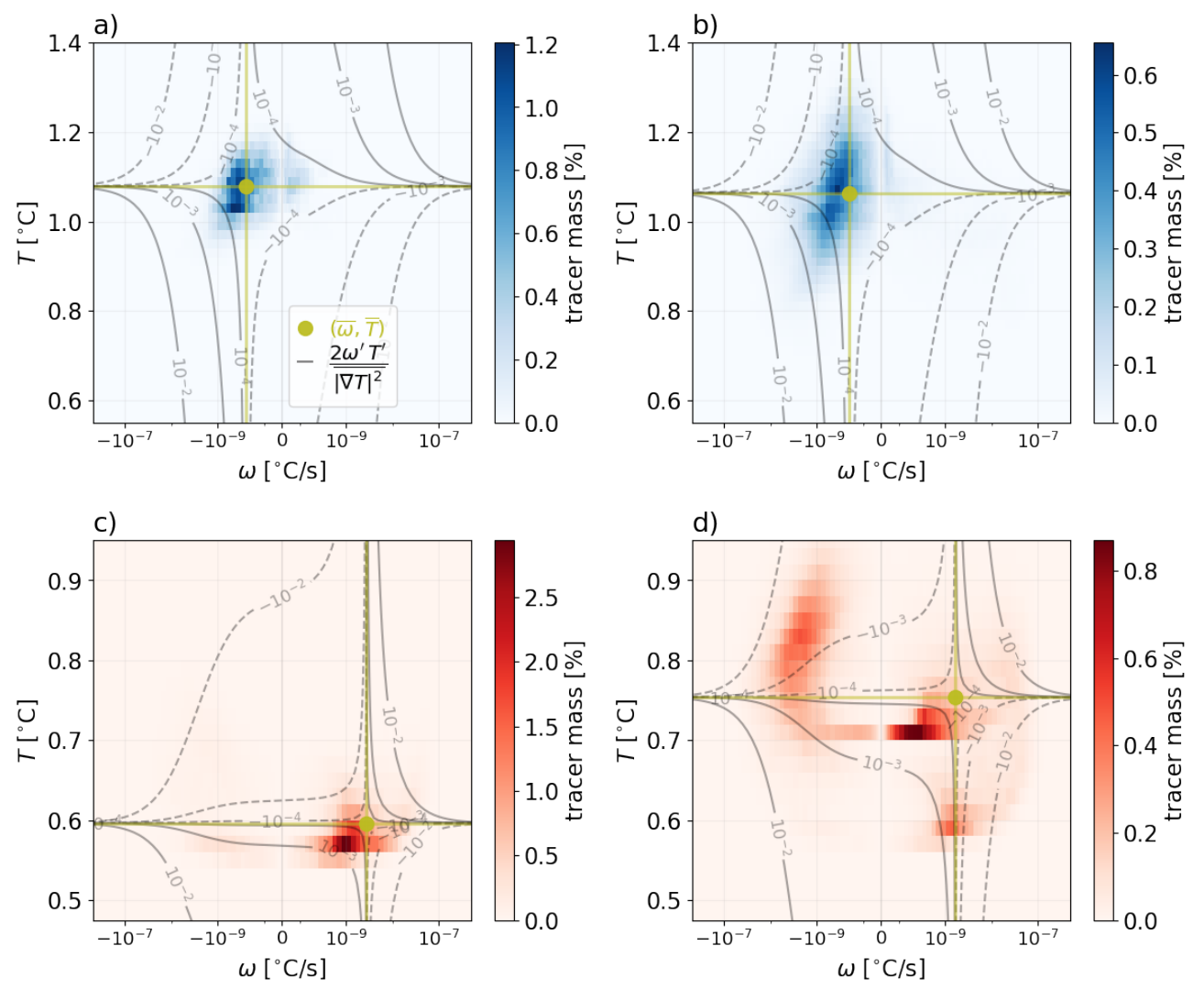

Figure B-3: Probability density function of tracer mass, as a percent contribution of each bin to the total tracer mass. Grey contours and olive lines as in Figure 4-8. 


\section{Bibliography}

Abbott, T. H. and Cronin, T. W. (2021). Aerosol invigoration of atmospheric convection through increases in humidity. Science, 371(6524):83-85. Publisher: American Association for the Ad vancement of Science Section: Report.

Abernathey, R., Ferreira, D., and Klocker, A. (2013). Diagnostics of isopycnal mixing in a circum polar channel. Ocean Modelling, 72:1-16.

Abernathey, R. P., Cerovecki, I., Holland, P. R., Newsom, E., Mazloff, M., and Talley, L. D. (2016). Water-mass transformation by sea ice in the upper branch of the Southern Ocean overturning. Nature Geoscience, 9(8):596-601. Publisher: Nature Publishing Group.

Alford, M. H., Girton, J. B., Voet, G., Carter, G. S., Mickett, J. B., and Klymak, J. M. (2013). Turbulent mixing and hydraulic control of abyssal water in the Samoan Passage: SAMOAN PASSAGE MIXING. Geophysical Research Letters, 40(17):4668-4674.

Alford, M. H., Peacock, T., MacKinnon, J. A., Nash, J. D., Buijsman, M. C., Centurioni, L. R., Chao, S.-Y., Chang, M.-H., Farmer, D. M., Fringer, O. B., Fu, K.-H., Gallacher, P. C., Graber, H. C., Helfrich, K. R., Jachec, S. M., Jackson, C. R., Klymak, J. M., Ko, D. S., Jan, S., Johnston, T. M. S., Legg, S., Lee, I.-H., Lien, R.-C., Mercier, M. J., Moum, J. N., Musgrave, R., Park, J.-H., Pickering, A. I., Pinkel, R., Rainville, L., Ramp, S. R., Rudnick, D. L., Sarkar, S., Scotti, A., Simmons, H. L., St Laurent, L. C., Venayagamoorthy, S. K., Wang, Y.-H., Wang, J., Yang, Y. J., Paluszkiewicz, T., and (David) Tang, T.-Y. (2015). The formation and fate of internal waves in the South China Sea. Nature, 521(7550):65-69. Bandiera_abtest: a Cg_type: Nature Research Journals Number: 7550 Primary_atype: Research Publisher: Nature Publishing Group Subject_term: Physical oceanography Subject_term_id: physical-oceanography.

Andersson, H. C. and Veronis, G. (2004). Thermohaline circulation in a two-layer model with sloping boundaries and a mid-ocean ridge. Deep Sea Research Part I: Oceanographic Research Papers, 51(1):93-106.

Archer, D., Kheshgi, H., and Maier-Reimer, E. (1998). Dynamics of fossil fuel CO 2 neutralization by marine $\mathrm{CaCO}_{3}$. Global Biogeochemical Cycles, 12(2):259-276.

Armi, L. (1977). The Dynamics of the Bottom Boundary Layer of the Deep Ocean*. In Nihoul, J. C. J., editor, Elsevier Oceanography Series, volume 19 of Bottom Turbulence, pages 153-164. Elsevier.

Armi, L. (1978). Some evidence for boundary mixing in the deep Ocean. Journal of Geophysical Research, 83(C4):1971.

Armi, L. (1979a). Effects of variations in eddy diffusivity on property distributions in the oceans. Journal of Marine Research, 37(3):515-530. 
Armi, L. (1979b). Reply to Comments by C. Garrett. Journal of Geophysical Research, 84(C8):5097. Publisher: Wiley-Blackwell.

Armi, L. and D'Asaro, E. (1980). Flow structures of the benthic ocean. Journal of Geophysical Research: Oceans, 85(C1):469-484. _eprint: https://agupubs.onlinelibrary.wiley.com/doi/pdf/10.1029/JC085iC01p00469.

Baines, P. G. (1979). Observations of stratified flow past Three-dimensional bar riers. Journal of Geophysical Research: Oceans, 84(C12):7834-7838. _eprint: https://agupubs.onlinelibrary.wiley.com/doi/pdf/10.1029/JC084iC12p07834.

Benthuysen, J. A. (2010). Linear and nonlinear stratified spindown over sloping topography. Mas sachusetts Institute of Technology and Woods Hole Oceanographic Institution, Woods Hole, MA.

Benthuysen, J. A. and Thomas, L. N. (2013). Nonlinear stratified spindown over a slope. Journal of Fluid Mechanics, 726:371-403.

Bernard, R. E. and Cooperdock, E. H. G. (2018). No progress on diversity in 40 years. Nature Geoscience, 11(5):292-295. Bandiera_abtest: a Cg_type: Nature Research Journals Number: 5 Primary_atype: Comments \& Opinion Publisher: Nature Publishing Group Subject_term: Atmospheric science;Careers;Education;Ocean sciences;Solid Earth sciences Subject_term_id: atmospheric-science;careers;education;ocean-sciences;solid-earth-sciences.

Bezanson, J., Edelman, A., Karpinski, S., and Shah, V. (2017). Julia: A Fresh Approach to Numerical Computing. SIAM Review, 59(1):65-98.

Boccaletti, G., Ferrari, R., and Fox-Kemper, B. (2007). Mixed Layer Instabilities and Restratifica tion. Journal of Physical Oceanography, 37(9):2228-2250.

Boucher, O., Servonnat, J., Albright, A. L., Aumont, O., Balkanski, Y., Bastrikov, V., Bekki, S., Bonnet, R., Bony, S., Bopp, L., Braconnot, P., Brockmann, P., Cadule, P., Caubel, A., Cheruy, F., Codron, F., Cozic, A., Cugnet, D., D'Andrea, F., Davini, P., Lavergne, C. d., Denvil, S., Deshayes, J., Devilliers, M., Ducharne, A., Dufresne, J.-L., Dupont, E., Éthé, C., Fairhead, L., Falletti, L., Flavoni, S., Foujols, M.-A., Gardoll, S., Gastineau, G., Ghattas, J., Grandpeix, J. Y., Guenet, B., Guez, E., L., Guilyardi, E., Guimberteau, M., Hauglustaine, D., Hourdin, F., Idelkadi, A., Joussaume, S., Kageyama, M., Khodri, M., Krinner, G., Lebas, N., Levavasseur, G., Lévy, C., Li, L., Lott, F., Lurton, T., Luyssaert, S., Madec, G., Madeleine, J.-B., Maig nan, F., Marchand, M., Marti, O., Mellul, L., Meurdesoif, Y., Mignot, J., Musat, I., Ottlé, C., Peylin, P., Planton, Y., Polcher, J., Rio, C., Rochetin, N., Rousset, C., Sepulchre, P., Sima, A., Swingedouw, D., Thiéblemont, R., Traore, A. K., Vancoppenolle, M., Vial, J., Vialard, J., Viovy, N., and Vuichard, N. (2020). Presentation and Evaluation of the IPSL-CM6A-LR Cli mate Model. Journal of Advances in Modeling Earth Systems, 12(7):e2019MS002010. _eprint: https://agupubs.onlinelibrary.wiley.com/doi/pdf/10.1029/2019MS002010.

Broecker, W. S. and Peng, T.-H. (1982). Tracers in the Sea. Lamont-Doherty Geological Observa tory, Palisades, New York.

Bryan, K. and Lewis, L. J. (1979). A water mass model of the world ocean. Journal of Geophysical Research, 84(C5):2503-2517. ISBN: 0148-0227.

Bryden, H. L. and Nurser, A. J. G. (2003). Effects of Strait Mixing on Ocean Stratification. Journal of Physical Oceanography, 33(8):1870-1872. Publisher: American Meteorological Society Section: Journal of Physical Oceanography. 
Burns, K. J., Vasil, G. M., Oishi, J. S., Lecoanet, D., and Brown, B. (2016). Dedalus: Flexible framework for spectrally solving differential equations. Astrophysics Source Code Library, record ascl:1603.015.

Cael, B. and Mashayek, A. (2021). Log-Skew-Normality of Ocean Turbulence. Physical Review Letters, 126(22):224502. Publisher: American Physical Society.

Callies, J. (2018). Restratification of Abyssal Mixing Layers by Submesoscale Baroclinic Eddies. Journal of Physical Oceanography, pages JPO-D-18-0082.1.

Callies, J. and Ferrari, R. (2018a). Baroclinic Instability in the Presence of Convection. Journal of Physical Oceanography, 48(1):45-60. Publisher: American Meteorological Society.

Callies, J. and Ferrari, R. (2018b). Dynamics of an Abyssal Circulation Driven by Bottom-Intensified Mixing on Slopes. Journal of Physical Oceanography, 48(6):1257-1282.

Callies, J. and Marotzke, J. (2012). A simple and self-consistent geostrophic-force-balance model of the thermohaline circulation with boundary mixing. Ocean Science, 8(1):49-63.

Cember, R. P. (1998). On deep western boundary currents. Journal of Geophysical Research: Oceans, 103(C3):5397-5417.

Chalamalla, V. K., Santilli, E., Scotti, A., Jalali, M., and Sarkar, S. (2017). SOMAR-LES: A frame work for multi-scale modeling of turbulent stratified oceanic flows. Ocean Modelling, 120:101-119.

Cimoli, L., Caulfield, C.-c. P., Johnson, H. L., Marshall, D. P., Mashayek, A., Garabato, A. C. N., and Vic, C. (2019). Sensitivity of deep ocean mixing to local internal tide breaking and mixing efficiency. Geophysical Research Letters, n/a(n/a).

Clément, L., Thurnherr, A. M., and St. Laurent, L. C. (2017). Turbulent Mixing in a Deep Fracture Zone on the Mid-Atlantic Ridge. Journal of Physical Oceanography, 47(8):1873-1896.

Danabasoglu, G., Large, W. G., and Briegleb, B. P. (2010). Climate impacts of parameter ized Nordic Sea overflows. Journal of Geophysical Research: Oceans, 115(C11). _eprint: https://agupubs.onlinelibrary.wiley.com/doi/pdf/10.1029/2010JC006243.

D'Asaro, E. (1982). Velocity Structure of the Benthic Ocean. Journal of Physical Oceanography, 12(4):313-322. Publisher: American Meteorological Society Section: Journal of Physical Oceanography.

de Lavergne, C., Madec, G., Capet, X., Maze, G., and Roquet, F. (2016a). Getting to the bottom of the ocean. Nature Geoscience, 9(12):857-858. Number: 12 Publisher: Nature Publishing Group.

de Lavergne, C., Madec, G., Le Sommer, J., Nurser, A. J. G., and Naveira Garabato, A. C. (2016b). The Impact of a Variable Mixing Efficiency on the Abyssal Overturning. Journal of Physical Oceanography, 46(2):663-681.

de Lavergne, C., Madec, G., Le Sommer, J., Nurser, A. J. G., and Naveira Garabato, A. C. (2016c). On the Consumption of Antarctic Bottom Water in the Abyssal Ocean. Journal of Physical Oceanography, 46(2):635-661.

de Lavergne, C., Madec, G., Roquet, F., Holmes, R. M., and McDougall, T. J. (2017). Abyssal ocean overturning shaped by seafloor distribution. Nature, 551(7679):181-186. Publisher: Nature Publishing Group. 
De Szoeke, R. A. and Bennett, A. F. (1993). Microstructure Fluxes across Density Surfaces. Journal of Physical Oceanography, 23(10):2254-2264. Publisher: American Meteorological Society.

Dell, R. and Pratt, L. (2015). Diffusive boundary layers over varying topography. Journal of Fluid Mechanics, 769:635-653.

Dell, R. W. (2013). Boundary layer dynamics and deep ocean mixing in Mid-Atlantic Ridge canyons. Massachusetts Institute of Technology and Woods Hole Oceanographic Institution, Woods Hole, MA.

Dillon, T. M. (1982). Vertical overturns: A comparison of Thorpe and Ozmidov length scales. Journal of Geophysical Research: Oceans, 87(C12):9601-9613. _eprint: https://agupubs.onlinelibrary.wiley.com/doi/pdf/10.1029/JC087iC12p09601.

Drake, H. (2020). hdrake/abyssalflow: First set of revisions.

Drake, H. F., Ferrari, R., and Callies, J. (2020a). Abyssal Circulation Driven by Near-Boundary Mixing: Water Mass Transformations and Interior Stratification. Journal of Physical Oceanography, 50(8):2203-2226. Publisher: American Meteorological Society.

Drake, H. F., Rivest, R. L., Deutch, J., and Edelman, A. (2020b). A multi-control climate policy process for a trusted decision maker. Publisher: EarthArXiv.

Durrieu De Madron, X. and Weatherly, G. (1994). Circulation, transport and bottom boundary layers of the deep currents in the Brazil Basin. Journal of Marine Research, 52(4):583-638.

Emile-Geay, J. and Madec, G. (2009). Geothermal heating, diapycnal mixing and the abyssal circulation. Ocean Science, 5(2):203-217.

Ferrari, R. (2014). What goes down must come up. Nature, 513:179-180.

Ferrari, R., Mashayek, A., McDougall, T. J., Nikurashin, M., and Campin, J.-M. (2016). Turning Ocean Mixing Upside Down. Journal of Physical Oceanography, 46(7):2239-2261.

Fox-Kemper, B., Danabasoglu, G., Ferrari, R., Griffies, S. M., Hallberg, R. W., Holland, M. M., Maltrud, M. E., Peacock, S., and Samuels, B. L. (2011). Parameterization of mixed layer eddies. III: Implementation and impact in global ocean climate simulations. Ocean Modelling, 39(1):6178.

Fox-Kemper, B., Ferrari, R., and Hallberg, R. (2008). Parameterization of Mixed Layer Eddies. Part I: Theory and Diagnosis. Journal of Physical Oceanography, 38(6):1145-1165.

Friedrich, T., Timmermann, A., Decloedt, T., Luther, D. S., and Mouchet, A. (2011). The ef fect of topography-enhanced diapycnal mixing on ocean and atmospheric circulation and marine biogeochemistry. Ocean Modelling, 39(3):262-274.

Garabato, A. C. N., Frajka-Williams, E. E., Spingys, C. P., Legg, S., Polzin, K. L., Forryan, A., Abrahamsen, E. P., Buckingham, C. E., Griffies, S. M., McPhail, S. D., Nicholls, K. W., Thomas, L. N., and Meredith, M. P. (2019). Rapid mixing and exchange of deep-ocean waters in an abyssal boundary current. Proceedings of the National Academy of Sciences, 116(27):13233-13238. ISBN: 9781904087113 Publisher: National Academy of Sciences Section: Physical Sciences.

Garrett, C. (1979). Comment on 'Some evidence for boundary mixing in the deep ocean' by Laurence Armi. Journal of Geophysical Research, 84(C8):5095. Publisher: Wiley-Blackwell. 
Garrett, C. (1990). The role of secondary circulation in boundary mixing. Journal of Geophysical Research, 95(C3):3181. Publisher: Wiley-Blackwell.

Garrett, C. (1991). Marginal mixing theories. Atmosphere-Ocean, 29(2):313-339.

Garrett, C. (2001). An Isopycnal View of Near-Boundary Mixing and Associated Flows. Journal of Physical Oceanography, 31(1):138-142.

Garrett, C., MacCready, P., and Rhines, P. (1993). Boundary Mixing and Arrested Ekman Layers: Rotating Stratified Flow Near a Sloping Boundary. Annual Review of Fluid Mechanics, 25(1):291323. Publisher: Annual Reviews 4139 El Camino Way, P.O. Box 10139, Palo Alto, CA 94303-0139, USA.

Garrett, C. and Munk, W. (1972). Space-Time scales of internal waves. Geophysical Fluid Dynamics, 3(3):225-264. Publisher: Taylor \& Francis_eprint: https://doi.org/10.1080/03091927208236082.

Garrett, C. and Munk, W. (1975). Space-time scales of internal waves: A progress report. Journal of Geophysical Research (1896-1977), 80(3):291-297. _eprint: https://agupubs.onlinelibrary.wiley.com/doi/pdf/10.1029/JC080i003p00291.

Gayen, B. and Sarkar, S. (2011). Boundary mixing by density overturns in an internal tidal beam. Geophysical Research Letters, 38(14). _eprint: https://agupubs.onlinelibrary.wiley.com/doi/pdf/10.1029/2011GL048135.

Gent, P. R. and McWilliams, J. C. (1990). Isopycnal Mixing in Ocean Circulation Models. Journal of Physical Oceanography, 20(1):150-155. ISBN: 0022-3670.

Geoffroy, O., Saint-Martin, D., Olivié, D. J. L., Voldoire, A., Bellon, G., and Tytéca, S. (2012). Transient Climate Response in a Two-Layer Energy-Balance Model. Part I: Analytical Solution and Parameter Calibration Using CMIP5 AOGCM Experiments. Journal of Climate, 26(6):18411857. Publisher: American Meteorological Society.

Gordon, A. L. (1986). Is there a global scale ocean circulation? Eos, Transactions American Geophysical Union, 67(9):109-110. _eprint: https://agupubs.onlinelibrary.wiley.com/doi/pdf/10.1029/EO067i009p00109.

Gouretski, V. and Koltermann, K. P. (2004). WOCE global hydrographic climatology. Berichte des BSH, 35:1-52.

Greatbatch, R. J. and Lamb, K. G. (1990). On Parameterizing Vertical Mixing of Momentum in Non-eddy Resolving Ocean Models. Journal of Physical Oceanography, 20(10):1634-1637.

Gregg, M., D’Asaro, E., Riley, J., and Kunze, E. (2018). Mixing Efficiency in the Ocean. Annual Review of Marine Science, 10(1):443-473.

Gregg, M. C. (1987). Diapycnal mixing in the thermocline: A review. Journal of Geophysical Research, 92(C5):5249.

Gregg, M. C. (1989). Scaling turbulent dissipation in the thermocline. Journal of Geophysical Research, 94(C7):9686.

Gregg, M. C., Sanford, T. B., and Winkel, D. P. (2003). Reduced mixing from the breaking of internal waves in equatorial waters. Nature, 422(6931):513-515. Number: 6931 Publisher: Nature Publishing Group. 
Groeskamp, S., Sloyan, B. M., Zika, J. D., and McDougall, T. J. (2017). Mixing Inferred from an Ocean Climatology and Surface Fluxes. Journal of Physical Oceanography, 47(3):667-687.

Haine, T. W. N. and Marshall, J. (1998). Gravitational, Symmetric, and Baroclinic Instability of the Ocean Mixed Layer. Journal of Physical Oceanography, 28(4):634-658. Publisher: American Meteorological Society Section: Journal of Physical Oceanography.

Hansen, J., Russell, G., Lacis, A., Fung, I., Rind, D., and Stone, P. (1985). Climate Response Times: Dependence on Climate Sensitivity and Ocean Mixing. Science, 229(4716):857-859.

Held, I. M. (2005). The gap between simulation and understanding in climate modeling. Bulletin of the American Meteorological Society, 86(11):1609-1614. ISBN: 0003-0007.

Henyey, F. S., Wright, J., and Flatté, S. M. (1986). Energy and action flow through the internal wave field: An eikonal approach. Journal of Geophysical Research: Oceans, 91(C7):8487-8495. _eprint: https://agupubs.onlinelibrary.wiley.com/doi/pdf/10.1029/JC091iC07p08487.

Hogg, N., Biscaye, P., Gardner, W., and Jr, W. (1982). On the Transport and Modification of Antarctic Bottom Water in the Vema Channel. J. Mar. Res., 40:231-263.

Holden, P. B., Edwards, N. R., Fraedrich, K., Kirk, E., Lunkeit, F., and Zhu, X. (2016). PLASIM-GENIE v1.0: a new intermediate complexity AOGCM. Geoscientific Model Development, 9(9):3347-3361.

Holmes, R. M., de Lavergne, C., and McDougall, T. J. (2018). Ridges, Seamounts, Troughs, and Bowls: Topographic Control of the Dianeutral Circulation in the Abyssal Ocean. Journal of Physical Oceanography, 48(4):861-882.

Holmes, R. M., de Lavergne, C., and McDougall, T. J. (2019). Tracer Transport within Abyssal Mixing Layers. Journal of Physical Oceanography, 49(10):2669-2695. Publisher: American Me teorological Society.

Holmes, R. M. and McDougall, T. J. (2020). Diapycnal Transport near a Sloping Bottom Boundary. Journal of Physical Oceanography, 50(11):3253-3266. Publisher: American Meteorological Society Section: Journal of Physical Oceanography.

Huang, R. X. and Jin, X. (2002). Deep Circulation in the South Atlantic Induced by BottomIntensified Mixing over the Midocean Ridge*. Journal of Physical Oceanography, 32(4):1150-1164.

Ijichi, T., Laurent, L. S., Polzin, K. L., and Toole, J. M. (2020). How Variable Is Mix ing Efficiency in the Abyss? Geophysical Research Letters, 47(7):e2019GL086813. _eprint: https://agupubs.onlinelibrary.wiley.com/doi/pdf/10.1029/2019GL086813.

Imberger, J. and Ivey, G. N. (1993). Boundary mixing in stratified reservoirs. Journal of Fluid Mechanics, 248(-1):477. Publisher: Cambridge University Press.

Jalali, M. and Sarkar, S. (2017). Large Eddy Simulation of Flow and Turbulence at the Steep Topography of Luzon Strait. Geophysical Research Letters, 44(18):9440-9448. _eprint: https://agupubs.onlinelibrary.wiley.com/doi/pdf/10.1002/2017GL074119.

Jansen, M. F. and Nadeau, L.-P. (2019). A Toy Model for the Response of the Residual Overturning Circulation to Surface Warming. Journal of Physical Oceanography, 49(5):1249-1268. Publisher: American Meteorological Society. 
Jayne, S. R. (2009). The Impact of Abyssal Mixing Parameterizations in an Ocean General Circu lation Model. Journal of Physical Oceanography, 39(7):1756-1775.

Joos, F., Roth, R., Fuglestvedt, J. S., Peters, G. P., Enting, I. G., von Bloh, W., Brovkin, V., Burke, E. J., Eby, M., Edwards, N. R., Friedrich, T., Frölicher, T. L., Halloran, P. R., Holden, P. B., Jones, C., Kleinen, T., Mackenzie, F. T., Matsumoto, K., Meinshausen, M., Plattner, G.-K., Reisinger, A., Segschneider, J., Shaffer, G., Steinacher, M., Strassmann, K., Tanaka, K., Timmermann, A., and Weaver, A. J. (2013). Carbon dioxide and climate impulse response functions for the computation of greenhouse gas metrics: a multi-model analysis. Atmospheric Chemistry and Physics, 13(5):2793-2825. Publisher: Copernicus GmbH.

Kaiser, B. E. (2020). Finescale abyssal turbulence: sources and modeling. Thesis, Massachusetts Institute of Technology. Accepted: 2020-10-18T21:45:36Z.

Katsman, C. A. (2006). Impacts of Localized Mixing and Topography on the Stationary Abyssal Circulation. Journal of Physical Oceanography, 36(8):1660-1671. Publisher: American Meteoro logical Society Section: Journal of Physical Oceanography.

Kawase, M. (1987). Establishment of Deep Ocean Circulation Driven by Deep-Water Production. Journal of Physical Oceanography, 17(12):2294-2317.

Klocker, A. and McDougall, T. J. (2010). Influence of the Nonlinear Equation of State on Global Estimates of Dianeutral Advection and Diffusion. Journal of Physical Oceanography, 40(8):16901709.

Klymak, J. M. and Legg, S. M. (2010). A simple mixing scheme for models that resolve breaking internal waves. Ocean Modelling, 33(3-4):224-234.

Koltermann, K. P., Gouretski, V., and Jancke, K. (2011). Hydrographic Atlas of the World Ocean Circulation Experiment (WOCE): Volume 3: Atlantic Ocean. National Oceanography Centre.

Kunze, E., MacKay, C., McPhee-Shaw, E. E., Morrice, K., Girton, J. B., and Terker, S. R. (2012). Turbulent Mixing and Exchange with Interior Waters on Sloping Boundaries. Journal of Physical Oceanography, 42(6):910-927.

Lavergne, C. d., Vic, C., Madec, G., Roquet, F., Waterhouse, A. F., Whalen, C. B., Cuypers, Y., Bouruet-Aubertot, P., Ferron, B., and Hibiya, T. (2020). A Parameterization of Local and Remote Tidal Mixing. Journal of Advances in Modeling Earth Systems, 12(5):e2020MS002065. _eprint: https://agupubs.onlinelibrary.wiley.com/doi/pdf/10.1029/2020MS002065.

Ledwell, J. R., Duda, T. F., Sundermeyer, M. A., and Seim, H. E. (2004). Mixing in a coastal environment: 1. A view from dye dispersion. Journal of Geophysical Research: Oceans, 109(C10). _eprint: https://agupubs.onlinelibrary.wiley.com/doi/pdf/10.1029/2003JC002194.

Ledwell, J. R., He, R., Xue, Z., DiMarco, S. F., Spencer, L. J., and Chapman, P. (2016). Dispersion of a tracer in the deep Gulf of Mexico. Journal of Geophysical Research: Oceans, 121(2):11101132. _eprint: https://agupubs.onlinelibrary.wiley.com/doi/pdf/10.1002/2015JC011405.

Ledwell, J. R. and Hickey, B. M. (1995). Evidence for enhanced boundary mixing in the Santa Monica Basin. Journal of Geophysical Research: Oceans, 100(C10):20665-20679._ _eprint: https://agupubs.onlinelibrary.wiley.com/doi/pdf/10.1029/94JC01182. 
Ledwell, J. R., Montgomery, E. T., Polzin, K. L., St. Laurent, L. C., Schmitt, R. W., and Toole, J. M. (2000). Evidence for enhanced mixing over rough topography in the abyssal ocean. Nature, 403(6766):179-182. Publisher: Nature Publishing Group.

Ledwell, J. R., Watson, A. J., and Law, C. S. (1993). Evidence for slow mixing across the pycnocline from an open-ocean tracer-release experiment. Nature, 364(6439):701-703. Publisher: Nature Publishing Group.

Lee, S., Gohari, S. M. I., and Sarkar, S. (2020). Direct numerical simulation of stratified Ekman layers over a periodic rough surface. Journal of Fluid Mechanics, 902. Publisher: Cambridge University Press.

Legg, S. (2021). Mixing by Oceanic Lee Waves. Annual Review of Fluid Mechanics, 53(1):173-201. _eprint: https://doi.org/10.1146/annurev-fluid-051220-043904.

Legg, S., Briegleb, B., Chang, Y., Chassignet, E. P., Danabasoglu, G., Ezer, T., Gordon, A. L., Griffies, S., Hallberg, R., Jackson, L., Large, W., Özgökmen, T. M., Peters, H., Price, J., Riemen schneider, U., Wu, W., Xu, X., and Yang, J. (2009). Improving Oceanic Overflow Representation in Climate Models: The Gravity Current Entrainment Climate Process Team. Bulletin of the American Meteorological Society, 90(5):657-670. Publisher: American Meteorological Society Section: Bulletin of the American Meteorological Society.

Levitus, S., Antonov, J. I., Boyer, T. P., Baranova, O. K., Garcia, H. E., Locarnini, R. A., Mishonov, A. V., Reagan, J. R., Seidov, D., Yarosh, E. S., and Zweng, M. M. (2012). World ocean heat content and thermosteric sea level change (0-2000 m), 1955-2010. Geophysical Research Letters, 39(10):n/a-n/a. Publisher: Wiley-Blackwell.

Lumpkin, R. and Speer, K. (2007). Global Ocean Meridional Overturning. Journal of Physical Oceanography, 37(10):2550-2562. ISBN: 0022-3670.

MacCready, P. and Rhines, P. B. (1991). Buoyant inhibition of Ekman transport on a slope and its effect on stratified spin-up. Journal of Fluid Mechanics, 223(-1):631. Publisher: Cambridge University Press.

Mackay, N., Ledwell, J. R., Messias, M.-J., Garabato, A. C. N., Brearley, J. A., Meijers, A. J. S., Jones, D. C., and Watson, A. J. (2018). Diapycnal Mix ing in the Southern Ocean Diagnosed Using the DIMES Tracer and Realistic Veloc ity Fields. Journal of Geophysical Research: Oceans, 123(4):2615-2634. _eprint: https://agupubs.onlinelibrary.wiley.com/doi/pdf/10.1002/2017JC013536.

MacKinnon, J. A., Zhao, Z., Whalen, C. B., Waterhouse, A. F., Trossman, D. S., Sun, O. M., St. Laurent, L. C., Simmons, H. L., Polzin, K., Pinkel, R., Pickering, A., Norton, N. J., Nash, J. D., Musgrave, R., Merchant, L. M., Melet, A. V., Mater, B., Legg, S., Large, W. G., Kunze, E., Klymak, J. M., Jochum, M., Jayne, S. R., Hallberg, R. W., Griffies, S. M., Diggs, S., Danabasoglu, G., Chassignet, E. P., Buijsman, M. C., Bryan, F. O., Briegleb, B. P., Barna, A., Arbic, B. K., Ansong, J. K., Alford, M. H., MacKinnon, J. A., Zhao, Z., Whalen, C. B., Waterhouse, A. F., Trossman, D. S., Sun, O. M., Laurent, L. C. S., Simmons, H. L., Polzin, K., Pinkel, R., Pickering, A., Norton, N. J., Nash, J. D., Musgrave, R., Merchant, L. M., Melet, A. V., Mater, B., Legg, S., Large, W. G., Kunze, E., Klymak, J. M., Jochum, M., Jayne, S. R., Hallberg, R. W., Griffies, S. M., Diggs, S., Danabasoglu, G., Chassignet, E. P., Buijsman, M. C., Bryan, F. O., Briegleb, B. P., Barna, A., Arbic, B. K., Ansong, J. K., and Alford, M. H. (2017). Climate Process 
Team on Internal Wave-Driven Ocean Mixing. Bulletin of the American Meteorological Society, 98(11):2429-2454.

Marchal, O. and Nycander, J. (2004). Nonuniform Upwelling in a Shallow-Water Model of the Antarctic Bottom Water in the Brazil Basin. Journal of Physical Oceanography, 34(11):24922513. Publisher: American Meteorological Society Section: Journal of Physical Oceanography.

Marotzke, J. (1997). Boundary Mixing and the Dynamics of Three-Dimensional Thermohaline Circulations. Journal of Physical Oceanography, 27(8):1713-1728.

Marshall, J., Hill, C., Perelman, L., and Adcroft, A. (1997). Hydrostatic, quasi-hydrostatic, and nonhydrostatic ocean modeling. Journal of Geophysical Research, 102(C3):5733. ISBN: 2156-2202.

Marshall, J., Jamous, D., and Nilsson, J. (1999). Reconciling thermodynamic and dynamic methods of computation of water-mass transformation rates. Deep-Sea Research Part I: Oceanographic Research Papers, 46(4):545-572. ISBN: 0967-0637.

Marshall, J. and Radko, T. (2003). Residual-Mean Solutions for the Antarctic Circumpolar Current and Its Associated Overturning Circulation. Journal of Physical Oceanography, 33(11):2341-2354. ISBN: 0022-3670.

Marshall, J. and Speer, K. (2012). Closure of the meridional overturning circulation through South ern Ocean upwelling. Nature Geoscience, 5(3):171-180. Publisher: Nature Publishing Group ISBN: 1752-0894.

Mashayek, A., Ferrari, R., Merrifield, S., Ledwell, J. R., St Laurent, L., and Garabato, A. N. (2017a). Topographic enhancement of vertical turbulent mixing in the Southern Ocean. Nature Communications, 8:14197. Publisher: Nature Publishing Group.

Mashayek, A., Ferrari, R., Nikurashin, M., and Peltier, W. R. (2015). Influence of Enhanced Abyssal Diapycnal Mixing on Stratification and the Ocean Overturning Circulation. Journal of Physical Oceanography, 45(10):2580-2597.

Mashayek, A., Salehipour, H., Bouffard, D., Caulfield, C. P., Ferrari, R., Nikurashin, M., Peltier, W. R., and Smyth, W. D. (2017b). Efficiency of turbulent mixing in the abyssal ocean circulation. Geophysical Research Letters, 44(12):6296-6306. Publisher: Wiley-Blackwell.

McDougall, T. J. (1989). Dianeutral advection. In Parameterization of Small-Scale Processes: Proc. 'Aha Huliko 'a Hawaiian Winter Workshop, pages 289-315.

McDougall, T. J. and Ferrari, R. (2017). Abyssal Upwelling and Downwelling Driven by NearBoundary Mixing. Journal of Physical Oceanography, 47(2):261-283. Publisher: American Me teorological Society.

McPhee-Shaw, E. E., Kunze, E., and Girton, J. B. (2021). Submarine Canyon Oxygen Anomaly Caused by Mixing and Boundary-Interior Ex change. Geophysical Research Letters, 48(10):e2021GL092995. _eprint: https://agupubs.onlinelibrary.wiley.com/doi/pdf/10.1029/2021GL092995.

Melet, A., Hallberg, R., Legg, S., and Nikurashin, M. (2014). Sensitivity of the Ocean State to Lee Wave-Driven Mixing. Journal of Physical Oceanography, 44(3):900-921. 
Melet, A., Hallberg, R., Legg, S., and Polzin, K. (2013). Sensitivity of the Ocean State to the Vertical Distribution of Internal-Tide-Driven Mixing. Journal of Physical Oceanography, 43(3):602-615.

Melet, A., Legg, S., and Hallberg, R. (2016). Climatic Impacts of Parameterized Local and Remote Tidal Mixing. Journal of Climate, 29(10):3473-3500.

Meredith, M. and Garabato, A. N. (2021). Ocean Mixing: Drivers, Mechanisms and Impacts. Elsevier Science. Google-Books-ID: e8syzgEACAAJ.

Montgomery, R. B. (1940). The Present Evidence on the Importance of Lateral Mixing Processes in the Ocean. Bulletin of the American Meteorological Society, 21(3):87-94. Publisher: American Meteorological Society Section: Bulletin of the American Meteorological Society.

Morris, M. Y., Hall, M. M., Laurent, L. C. S., and Hogg, N. G. (2001). Abyssal Mixing in the Brazil Basin. Journal of Physical Oceanography, 31(11):3331-3348. Publisher: American Meteorological Society Section: Journal of Physical Oceanography.

Morris, V. R. (2021). Combating Racism in the Geosciences: Reflections From a Black Professor. AGU Advances, 2(1):e2020AV000358. _eprint: https://agupubs.onlinelibrary.wiley.com/doi/pdf/10.1029/2020AV000358.

Mouw, C. B., Clem, S., Legg, S., and Stockard, J. (2018). Meeting Mentoring Needs in Physical Oceanography: An Evaluation of the Impact of MPOWIR. Oceanography, 31(4):171-179.

Munk, W. H. (1966). Abyssal recipes. Deep Sea Research and Oceanographic Abstracts, 13(4):707730. arXiv: cs/9605103 ISBN: 1600117471.

Munk, W. H. and Wunsch, C. (1998). Abyssal Recipes II: energetics of tidal and wind mixing. Deep-Sea Research Part I: Oceanographic Research Papers, 45:1978-2010.

Nikurashin, M. and Ferrari, R. (2009). Radiation and Dissipation of Internal Waves Generated by Geostrophic Motions Impinging on Small-Scale Topography: Theory. Journal of Physical Oceanography, 40(5):1055-1074.

Nikurashin, M. and Ferrari, R. (2011). Global energy conversion rate from geostrophic flows into internal lee waves in the deep ocean. Geophysical Research Letters, 38(8).

Nikurashin, M. and Ferrari, R. (2013). Overturning circulation driven by breaking internal waves in the deep ocean. Geophysical Research Letters, 40(12):3133-3137. Publisher: Wiley-Blackwell.

Nikurashin, M. and Legg, S. (2011). A Mechanism for Local Dissipation of Internal Tides Generated at Rough Topography. Journal of Physical Oceanography, 41(2):378-395.

Nikurashin, M. and Vallis, G. (2011). A Theory of Deep Stratification and Overturning Circulation in the Ocean. Journal of Physical Oceanography, 41(3):485-502.

Nikurashin, M., Vallis, G., Nikurashin, M., and Vallis, G. (2012). A Theory of the Interhemi spheric Meridional Overturning Circulation and Associated Stratification. Journal of Physical Oceanography, 42(10):1652-1667.

Nycander, J. (2005). Generation of internal waves in the deep ocean by tides. Journal of Geophysical Research: Oceans, 110(C10). 
Oreskes, N. (2021). Science on a Mission: How Military Funding Shaped What We Do and Don't Know about the Ocean. University of Chicago Press, first edition edition.

Orszag, S. A. (1970). Analytical theories of turbulence. Journal of Fluid Mechanics, 41(2):363-386. Publisher: Cambridge University Press.

Osborn, T. R. (1980). Estimates of the Local Rate of Vertical Diffusion from Dissipation Measure ments. Journal of Physical Oceanography, 10(1):83-89.

Osborn, T. R. and Cox, C. S. (1972). Oceanic fine structure. Geophysical Fluid Dynamics, 3(1):321345.

Peacock, T. and Alford, M. H. (2018). Is deep-sea mining worth it? Scientific American, 318(5):7277.

Pedlosky, J. (1965). A note on the western intensification of the oceanic circulation. Journal of Marine Research, pages 207-209.

Pedlosky, J. (1987). Geophysical Fluid Dynamics. Springer-Verlag, New York, 2 edition.

Pedlosky, J. (1992). The Baroclinic Structure of the Abyssal Circulation. Journal of Physical Oceanography, 22(6):652-659.

Pedlosky, J. (1996). Ocean Circulation Theory. Springer Berlin Heidelberg, Berlin, Heidelberg.

Phillips, O. (1970). On flows induced by diffusion in a stably stratified fluid. Deep Sea Research and Oceanographic Abstracts, 17(3):435-443.

Phillips, O. M., Shyu, J.-H., and Salmun, H. (1986). An experiment on boundary mixing: mean circulation and transport rates. Journal of Fluid Mechanics, 173(-1):473.

Polzin, K. (2004). A Heuristic Description of Internal Wave Dynamics. Journal of Physical Oceanography, 34(1):214-230. Publisher: American Meteorological Society Section: Journal of Physical Oceanography.

Polzin, K., Toole, J., Ledwell, J. R., and Schmitt, R. (1997). Spatial Variability of Turbulent Mixing in the Spatial Variability Abyssal Ocean. Science, 276(5309):93-96. ISBN: 0036-8075.

Polzin, K. L. (2009). An abyssal recipe. Ocean Modelling, 30(4):298-309.

Polzin, K. L., Speer, K. G., Toole, J. M., and Schmitt, R. W. (1996). Intense mixing of Antarctic Bottom Water in the equatorial Atlantic Ocean. Nature, 380(6569):54-57. Bandiera_abtest: a Cg_type: Nature Research Journals Number: 6569 Primary_atype: Research Publisher: Nature Publishing Group.

Polzin, K. L., Toole, J. M., Schmitt, R. W., Polzin, K. L., Toole, J. M., and Schmitt, R. W. (1995). Finescale Parameterizations of Turbulent Dissipation. Journal of Physical Oceanography, $25(3): 306-328$.

Polzin, K. L., Wang, B., Wang, Z., Thwaites, F., and Williams, A. J. (2021). Moored Flux and Dissipation Estimates from the Northern Deepwater Gulf of Mexico. Fluids, 6(7):237. Number: 7 Publisher: Multidisciplinary Digital Publishing Institute. 
Pratt, L. and Whitehead, J. (2008). Rotating Hydraulics: Nonlinear Topographic Effects in the Ocean and Atmosphere.

Radko, T. (2020). Control of baroclinic instability by submesoscale topography. Journal of Fluid Mechanics, 882:A14.

Ramadhan, A., Wagner, G. L., Hill, C., Campin, J.-M., Churavy, V., Besard, T., Souza, A., Edel man, A., Ferrari, R., and Marshall, J. (2020). Oceananigans.jl: Fast and friendly geophysical fluid dynamics on GPUs. Journal of Open Source Software, 5(53):2018.

Ranganathan, M., Lalk, E., Freese, L. M., Freilich, M. A., Wilcots, J., Duffy, M. L., and Shivamoggi, R. (2021). Trends in the representation of women amongst geoscience faculty from 1999-2020: the long road towards gender parity. Archive Location: world Publisher: Earth and Space Science Open Archive Section: Education.

Redi, M. H. (1982). Oceanic Isopycnal Mixing by Coordinate Rotation. Journal of Physical Oceanography, 12(10):1154-1158. Publisher: American Meteorological Society Section: Journal of Physi cal Oceanography.

Resing, J. A., Sedwick, P. N., German, C. R., Jenkins, W. J., Moffett, J. W., Sohst, B. M., and Tagliabue, A. (2015). Basin-scale transport of hydrothermal dissolved metals across the South Pacific Ocean. Nature, 523(7559):200-203. Number: 7559 Publisher: Nature Publishing Group.

Rhines, P. B. (1993). Oceanic General Circulation: Wave and Advection Dynamics. In Willebrand, J. and Anderson, D. L. T., editors, Modelling Oceanic Climate Interactions, pages 67-149, Berlin, Heidelberg. Springer Berlin Heidelberg.

Rhines, P. B. and Young, W. R. (1982). Homogenization of potential vorticity in planetary gyres. Journal of Fluid Mechanics, 122(-1):347.

Robinson, A. and Stommel, H. (1959). The Oceanic Thermocline and the Associated Thermohaline Circulation. Tellus, 11(3):295-308. Publisher: Wiley/Blackwell (10.1111).

Ruan, X. and Callies, J. (2020). Mixing-Driven Mean Flows and Submesoscale Eddies over MidOcean Ridge Flanks and Fracture Zone Canyons. Journal of Physical Oceanography, 50(1):175195. Publisher: American Meteorological Society Section: Journal of Physical Oceanography.

Ruan, X. and Ferrari, R. (2021). Diagnosing Diapycnal Mixing from Passive Tracers. Journal of Physical Oceanography, 51(3):757-767. Publisher: American Meteorological Society Section: Journal of Physical Oceanography.

Ruan, X., Thompson, A. F., and Taylor, J. R. (2019). The Evolution and Arrest of a Turbulent Stratified Oceanic Bottom Boundary Layer over a Slope: Downslope Regime. Journal of Physical Oceanography, 49(2):469-487. Publisher: American Meteorological Society Section: Journal of Physical Oceanography.

Saenko, O. A. and Merryfield, W. J. (2005). On the Effect of Topographically Enhanced Mixing on the Global Ocean Circulation. Journal of Physical Oceanography, 35(5):826-834.

Salmon, R. (1992). A two-layer Gulf Stream over a continental slope. Journal of Marine Research, $50(3): 341-365$. 
Salmun, H., Killworth, P. D., and Blundell, J. R. (1991). A two-dimensional model of boundary mixing. Journal of Geophysical Research: Oceans, 96(C10):18447-18474.

Samelson, R. M. (1998). Large-Scale Circulation with Locally Enhanced Vertical Mixing*. Journal of Physical Oceanography, 28(4):712-726.

Sarmiento, J. L. and Gruber, N. (2006). Ocean biogeochemical dynamics. Princeton University Press.

Sarmiento, J. L. and Toggweiler, J. R. (1984). A new model for the role of the oceans in determining atmospheric PCO2. Nature, 308(5960):621-624. Publisher: Nature Publishing Group.

Schneider, S. H. and Thompson, S. L. (1981). Atmospheric CO2 and climate: Importance of the transient response. Journal of Geophysical Research: Oceans, 86(C4):3135-3147. _eprint: https://agupubs.onlinelibrary.wiley.com/doi/pdf/10.1029/JC086iC04p03135.

Scott, J. R. and Marotzke, J. (2002). The Location of Diapycnal Mixing and the Meridional Over turning Circulation. Journal of Physical Oceanography, 32(12):3578-3595. Publisher: American Meteorological Society Section: Journal of Physical Oceanography.

Sheen, K. L., Brearley, J. A., Garabato, A. C. N., Smeed, D. A., Waterman, S., Ledwell, J. R., Meredith, M. P., Laurent, L. S., Thurnherr, A. M., Toole, J. M., and Watson, A. J. (2013). Rates and mechanisms of turbulent dissipation and mixing in the South ern Ocean: Results from the Diapycnal and Isopycnal Mixing Experiment in the South ern Ocean (DIMES). Journal of Geophysical Research: Oceans, 118(6):2774-2792. _eprint: https://agupubs.onlinelibrary.wiley.com/doi/pdf/10.1002/jgrc.20217.

Siegel, D. A., DeVries, T., Doney, S., and Bell, T. (2021). Assessing the sequestration time scales of some ocean-based carbon dioxide reduction strategies. Environmental Research Letters.

Simmons, H. L., Jayne, S. R., St. Laurent, L. C., and Weaver, A. J. (2004). Tidally driven mixing in a numerical model of the ocean general circulation. Ocean Modelling, 6(3-4):245-263. ISBN: 1463-5003.

Smith, W. H. F. and Sandwell, D. T. (1997). Global Sea Floor Topography from Satellite Altimetry and Ship Depth Soundings. Science, 277(5334):1956-1962. Publisher: American Association for the Advancement of Science Section: Research Article.

Sobel, A. H., Nilsson, J., and Polvani, L. M. (2001). The Weak Temperature Gradient Approxima tion and Balanced Tropical Moisture Waves. Journal of the Atmospheric Sciences, 58(23):36503665. Publisher: American Meteorological Society Section: Journal of the Atmospheric Sciences.

Spall, M. A. (2001). Large-Scale Circulations Forced by Localized Mixing over a Sloping Bottom*. Journal of Physical Oceanography, 31(8):2369-2384.

Spingys, C. P., Garabato, A. C. N., Legg, S., Polzin, K. L., Abrahamsen, E. P., Buckingham, C. E., Forryan, A., and Frajka-Williams, E. E. (2021). Mixing and Transformation in a Deep Western Boundary Current: A Case Study. Journal of Physical Oceanography, 51(4):1205-1222. Publisher: American Meteorological Society Section: Journal of Physical Oceanography.

St. Laurent, L. and Garrett, C. (2002). The Role of Internal Tides in Mixing the Deep Ocean. Journal of Physical Oceanography, 32(10):2882-2899. 
St. Laurent, L. C., Toole, J. M., and Schmitt, R. W. (2001). Buoyancy Forcing by Turbulence above Rough Topography in the Abyssal Brazil Basin*. Journal of Physical Oceanography, 31(12):34763495 .

Stommel, H. (1948). The westward intensification of wind-driven ocean currents. Transactions, American Geophysical Union, 29(2):202.

Stommel, H. (1957). The Abyssal Circulation of the Ocean. Nature, 180(4589):733-734.

Stommel, H. (1958). The abyssal circulation. Deep Sea Research (1953), 5(1):80-82.

Stommel, H. (1961). Thermohaline Convection with Two Stable Regimes of Flow. Tellus, 13(2):224230. _eprint: https://onlinelibrary.wiley.com/doi/pdf/10.1111/j.2153-3490.1961.tb00079.x.

Stommel, H. and Arons, A. B. (1959a). On the abyssal circulation of the world ocean - II. An idealized model of the circulation pattern and amplitude in oceanic basins. Deep Sea Research (1953), 6:217-233.

Stommel, H. and Arons, A. B. (1959b). On the abyssal circulation of the world ocean-I. Stationary planetary flow patterns on a sphere. Deep Sea Research (1953), 6:140-154.

Stommel, H., Arons, A. B., and Faller, A. J. (1958). Some Examples of Stationary Planetary Flow Patterns in Bounded Basins. Tellus, 10(2):179-187.

Sverdrup, H., Johnson, M., and Fleming, R. (1942). The Oceans: Their Physics, Chemistry and General Biology. Oceanography, page 1104. arXiv: 1011.1669v3 ISBN: 9788578110796.

Szoeke, R. A. D. (1983). Baroclinic instability over wavy topography. Journal of Fluid Mechanics, 130:279-298. Publisher: Cambridge University Press.

Tagliabue, A., Aumont, O., DeAth, R., Dunne, J. P., Dutkiewicz, S., Galbraith, E., Misumi, K., Moore, J. K., Ridgwell, A., Sherman, E., Stock, C., Vichi, M., Völker, C., and Yool, A. (2016). How well do global ocean biogeochemistry models simulate dissolved iron distributions? Global Biogeochemical Cycles, 30(2):149-174. _ eprint: https://agupubs.onlinelibrary.wiley.com/doi/pdf/10.1002/2015GB005289.

Tagliabue, A., Bopp, L., Dutay, J.-C., Bowie, A. R., Chever, F., Jean-Baptiste, P., Bucciarelli, E., Lannuzel, D., Remenyi, T., Sarthou, G., Aumont, O., Gehlen, M., and Jeandel, C. (2010). Hydrothermal contribution to the oceanic dissolved iron inventory. Nature Geoscience, 3(4):252256. Number: 4 Publisher: Nature Publishing Group.

Talley, L. D. (2007). Hydrographic atlas of the world ocean circulation experiment (WOCE): Volume 2: Pacific Ocean. WOCE International Project Office Southampton.

Talley, L. D. (2013a). Closure of the Global Overturning Circulation Through the Indian, Pa cific, and Southern Oceans: Schematics and Transports. Oceanography, 26(1):80-97. arXiv: 1011.1669v3 ISBN: 1042-8275.

Talley, L. D. (2013b). Hydrographic Atlas of the World Ocean Circulation Experiment (WOCE): volume 4: Indian Ocean. International WOCE Project Office.

Talley, L. D., Reid, J. L., and Robbins, P. E. (2003). Data-Based Meridional Overturning Streamfunctions for the Global Ocean. Journal of Climate, 16(19):3213-3226. Publisher: American Meteorological Society Section: Journal of Climate. 
Tamsitt, V., Abernathey, R. P., Mazloff, M. R., Wang, J., and Talley, L. D. (2018). Transformation of Deep Water Masses Along Lagrangian Upwelling Pathways in the Southern Ocean: SOUTH ERN OCEAN UPWELLING TRANSFORMATION. Journal of Geophysical Research: Oceans, 123(3):1994-2017.

Taylor, G. I. (1922). Diffusion by Continuous Movements. Proceedings of the London Mathematical Society, s2-20(1):196-212. _eprint: https://londmathsoc.onlinelibrary.wiley.com/doi/pdf/10.1112/plms/s2-20.1.196.

Thompson, A. F., Stewart, A. L., and Bischoff, T. (2016). A Multibasin Residual-Mean Model for the Global Overturning Circulation. Journal of Physical Oceanography, 46(9):2583-2604.

Thompson, L. and Johnson, G. C. (1996). Abyssal currents generated by diffusion and geothermal heating over rises. Deep Sea Research Part I: Oceanographic Research Papers, 43(2):193-211.

Thorpe, S. A. (1987). Current and Temperature Variability on the Continental Slope. Philosophical Transactions of the Royal Society A: Mathematical, Physical and Engineering Sciences, 323(1574):471-517. Publisher: The Royal Society.

Thurnherr, A. M., Clément, L., Laurent, L. S., Ferrari, R., and Ijichi, T. (2020). Transforma tion and Upwelling of Bottom Water in Fracture Zone Valleys. Journal of Physical Oceanography, 50(3):715-726. Publisher: American Meteorological Society Section: Journal of Physical Oceanography.

Thurnherr, A. M. and Speer, K. G. (2003). Boundary Mixing and Topographic Blocking on the Mid-Atlantic Ridge in the South Atlantic*. Journal of Physical Oceanography, 33(4):848-862.

Thurnherr, A. M., St. Laurent, L. C., Speer, K. G., Toole, J. M., Ledwell, J. R., Thurnherr, A. M., Laurent, L. C. S., Speer, K. G., Toole, J. M., and Ledwell, J. R. (2005). Mixing Associated with Sills in a Canyon on the Midocean Ridge Flank*. Journal of Physical Oceanography, 35(8):13701381.

Toggweiler, J. R., Dixon, K., and Bryan, K. (1989). Simulations of radiocarbon in a coarse-resolution world ocean model: 1. Steady state prebomb distributions. Journal of Geophysical Research, $94(\mathrm{C} 6): 8217$.

Toole, J. M. (2007). Temporal Characteristics of Abyssal Finescale Motions above Rough Bathymetry. Journal of Physical Oceanography, 37(3):409-427. Publisher: American Meteo rological Society Section: Journal of Physical Oceanography.

Toole, J. M., Schmitt, R. W., and Polzin, K. L. (1994). Estimates of Diapycnal Mixing in the Abyssal Ocean. Science, 264(5162):1120-1123.

Tozer, B., Sandwell, D. T., Smith, W. H. F., Olson, C., Beale, J. R., and Wessel, P. (2019). Global Bathymetry and Topography at 15 Arc Sec: SRTM15+. Earth and Space Science, 6(10):18471864. _eprint: https://agupubs.onlinelibrary.wiley.com/doi/pdf/10.1029/2019EA000658.

Trossman, D., Whalen, C., Haine, T., Waterhouse, A., Bigdeli, A., Nguyen, A., Mazloff, M., He imbach, P., and Kovach, R. (2020). Tracer and observationally-derived constraints on horizontal and diapycnal diffusivities in ocean models. Archive Location: world Publisher: Earth and Space Science Open Archive Section: Oceanography. 
Trowbridge, J. H. and Lentz, S. J. (2018). The Bottom Boundary Layer. Annual Review of Marine Science, 10(1):397-420. _eprint: https://doi.org/10.1146/annurev-marine-121916-063351.

Tulloch, R., Ferrari, R., Jahn, O., Klocker, A., LaCasce, J., Ledwell, J. R., Marshall, J., Messias, M.-J., Speer, K., and Watson, A. (2014). Direct Estimate of Lateral Eddy Diffusivity Upstream of Drake Passage. Journal of Physical Oceanography, 44(10):2593-2616. Publisher: American Meteorological Society Section: Journal of Physical Oceanography.

Tziperman, E. (1986). On the Role of Interior Mixing and Air-Sea Fluxes in Determining the Stratification and Circulation of the Oceans. Journal of Physical Oceanography, 16(4):680-693. Publisher: American Meteorological Society Section: Journal of Physical Oceanography.

Visbeck, M., Dengler, M., Tanhua, T. S., and Freund, M. (2020). Mixing and Upwelling Dynamics along the Continental Slope off Peru inferred from Tracer Release, Hydrographic and Microstruc ture Measurements. AGU.

Voet, G., Girton, J. B., Alford, M. H., Carter, G. S., Klymak, J. M., and Mickett, J. B. (2015). Pathways, Volume Transport, and Mixing of Abyssal Water in the Samoan Passage. Journal of Physical Oceanography, 45(2):562-588. Publisher: American Meteorological Society Section: Journal of Physical Oceanography.

Walin, G. (1982). On the relation between sea-surface heat flow and thermal circulation in the ocean. Tellus, 34(2):187-195.

Waterhouse, A. F., MacKinnon, J. a., Nash, J. D., Alford, M. H., Kunze, E., Simmons, H. L., Polzin, K. L., St. Laurent, L. C., Sun, O. M., Pinkel, R., Talley, L. D., Whalen, C. B., Huussen, T. N., Carter, G. S., Fer, I., Waterman, S., Naveira Garabato, A. C., Sanford, T. B., and Lee, C. M. (2014). Global Patterns of Diapycnal Mixing from Measurements of the Turbulent Dissipation Rate. Journal of Physical Oceanography, 44(7):1854-1872. ISBN: 0022-3670.

Watson, A. J., Ledwell, J. R., Messias, M.-J., King, B. A., Mackay, N., Meredith, M. P., Mills, B., and Naveira Garabato, A. C. (2013). Rapid cross-density ocean mixing at mid-depths in the Drake Passage measured by tracer release. Nature, 501(7467):408-411. Publisher: Nature Publishing Group.

Watson, A. J., Ledwell, J. R., Webb, D. J., and Wunsch, C. (1988). Purposefully Released Tracers [and Discussion]. Philosophical Transactions of the Royal Society of London. Series A, Mathematical and Physical Sciences, 325(1583):189-200. Publisher: The Royal Society.

Weijer, W., Cheng, W., Drijfhout, S. S., Fedorov, A. V., Hu, A., Jackson, L. C., Liu, W., McDonagh, E. L., Mecking, J. V., and Zhang, J. (2019). Stability of the Atlantic Meridional Overturning Circulation: A Review and Synthesis. Journal of Geophysical Research: Oceans, 124(8):53365375. _eprint: https://agupubs.onlinelibrary.wiley.com/doi/pdf/10.1029/2019JC015083.

Wenegrat, J. O., Callies, J., and Thomas, L. N. (2018). Submesoscale Baroclinic Instability in the Bottom Boundary Layer. Journal of Physical Oceanography, pages JPO-D-17-0264.1.

Wenegrat, J. O. and Thomas, L. N. (2020). Centrifugal and Symmetric Instability during Ekman Adjustment of the Bottom Boundary Layer. Journal of Physical Oceanography, 50(6):1793-1812. Publisher: American Meteorological Society Section: Journal of Physical Oceanography. 
Whalen, C. B., de Lavergne, C., Naveira Garabato, A. C., Klymak, J. M., MacKinnon, J. A., and Sheen, K. L. (2020). Internal wave-driven mixing: governing processes and consequences for climate. Nature Reviews Earth $\&$ Environment, 1(11):606-621. Number: 11 Publisher: Nature Publishing Group.

Winters, K. B. and Armi, L. (2012). Hydraulic control of continuously stratified flow over an obstacle. Journal of Fluid Mechanics, 700:502-513. Publisher: Cambridge University Press.

Winters, K. B., Lombard, P. N., Riley, J. J., and D'Asaro, E. A. (1995). Available potential energy and mixing in density-stratified fluids. Journal of Fluid Mechanics, 289:115-128. Publisher: Cambridge University Press.

Wolfe, C. L. and Cessi, P. (2011). The Adiabatic Pole-to-Pole Overturning Circulation. Journal of Physical Oceanography, 41(9):1795-1810. Publisher: American Meteorological Society Section: Journal of Physical Oceanography.

Woods, A. W. (1991). Boundary-driven mixing. Journal of Fluid Mechanics, 226:625-654. Publisher: Cambridge University Press.

Wunsch, C. (1970). On oceanic boundary mixing. Deep-Sea Research and Oceanographic Abstracts, $17(2): 293-301$.

Wunsch, C. and Ferrari, R. (2004). VERTICAL MIXING, ENERGY, AND THE GENERAL CIR CUlAtion OF THE OCEANS. Annual Review of Fluid Mechanics, 36(1):281-314. Publisher: Annual Reviews.

Yang, X., Tziperman, E., and Speer, K. (2020). Dynamics of Deep Ocean East ern Boundary Currents. Geophysical Research Letters, 47(1):e2019GL085396. _ _eprint: https://agupubs.onlinelibrary.wiley.com/doi/pdf/10.1029/2019GL085396.

Yang, X., Tziperman, E., and Speer, K. (2021). Deep Eastern Boundary Currents: Idealized Models and Dynamics. Journal of Physical Oceanography, 51(4):989-1005. Publisher: American Meteo rological Society Section: Journal of Physical Oceanography.

Yankovsky, E. and Legg, S. (2019). Symmetric and Baroclinic Instability in Dense Shelf Overflows. Journal of Physical Oceanography, 49(1):39-61. Publisher: American Meteorological Society.

Yankovsky, E., Link to external site, t. l. w. o. i. a. n. w., Legg, S., Link to external site, t. 1. w. o. i. a. n. w., and Hallberg, R. W. (2020). Parameterization of submesoscale symmetric instability in dense flows along topography. Earth and Space Science Open Archive ESSOAr; Washington. Place: Washington, United States, Washington Publisher: American Geophysical Union Section: Oceanography.

Yi, Y. R., Legg, S., and Nazarian, R. H. (2017). The Impact of Topographic Steepness on Tidal Dissipation at Bumpy Topography. Fluids, 2(4):55. Number: 4 Publisher: Multidisciplinary Digital Publishing Institute.

Young, W. R. (2011). An Exact Thickness-Weighted Average Formulation of the Boussinesq Equa tions. Journal of Physical Oceanography, 42(5):692-707. 